\title{
The Anoxia Resistant Crayfish: A Profile of Stress Response
}

\author{
Benjamin Lant
}

B.Sc. Carleton University

A Thesis Submitted to the Faculty of Graduate and Post-Doctoral Affairs in partial fulfillment

of the requirements for the degree of

\author{
Doctor of Philosophy \\ Department of Biology \\ Carleton University \\ Ottawa, Ontario, Canada
}

(C) copyright 2011

Benjamin Lant 
Library and Archives

Canada

Published Heritage

Branch

395 Wellington Street

Ottawa ON K1A ON4

Canada
Bibliothèque et

Archives Canada

Direction du

Patrimoine de l'édition

395 , rue Wellington

Ottawa ON K1A ON4

Canada
Your file Votre référence

ISBN: 978-0-494-87764-7

Our file Notre référence

ISBN: $978-0-494-87764-7$

\section{NOTICE:}

The author has granted a nonexclusive license allowing Library and Archives Canada to reproduce, publish, archive, preserve, conserve, communicate to the public by telecommunication or on the Internet, loan, distrbute and sell theses worldwide, for commercial or noncommercial purposes, in microform, paper, electronic and/or any other formats.

The author retains copyright ownership and moral rights in this thesis. Neither the thesis nor substantial extracts from it may be printed or otherwise reproduced without the author's permission.
AVIS:

L'auteur a accordé une licence non exclusive permettant à la Bibliothèque et Archives Canada de reproduire, publier, archiver, sauvegarder, conserver, transmettre au public par télécommunication ou par l'Internet, prêter, distribuer et vendre des thèses partout dans le monde, à des fins commerciales ou autres, sur support microforme, papier, électronique et/ou autres formats.

L'auteur conserve la propriété du droit d'auteur et des droits moraux qui protege cette thèse. $\mathrm{Ni}$ la thèse ni des extraits substantiels de celle-ci ne doivent être imprimés ou autrement reproduits sans son autorisation.
In compliance with the Canadian Privacy Act some supporting forms may have been removed from this thesis.

While these forms may be included in the document page count, their removal does not represent any loss of content from the thesis.
Conformément à la loi canadienne sur la protection de la vie privée, quelques formulaires secondaires ont été enlevés de cette thèse.

Bien que ces formulaires aient inclus dans la pagination, il n'y aura aucun contenu manquant. 


\begin{abstract}
The capacity to adapt to and survive oxygen deprivation has long been an important topic of study, in both ecological and medical fields. The freshwater crayfish, Orconectes virilis, is capable of resisting anoxia, but the metabolic mechanisms underlying this are largely un-profiled. This thesis examines the activity and regulation of a number of stress response pathways in response to anoxia in $O$. virilis. The model organism Caenorhabditis elegans that shows stress-induced entry into hypometabolism (the dauer
\end{abstract} stage) was used as a template for selecting stress response pathways that could be important in crayfish anaerobiosis. The Akt signaling response showed a distinct increase in activity in crayfish tail muscle and hepatopancreas under anoxia, as assessed through phosphorylation states of the kinase and its downstream targets. This implicated a prosurvival response that functions by preventing cell cycle attenuation. Despite elevated Akt activity, residual FoxO activity remained, possibly mediating a pro-survival mechanism through transactivation of antioxidant genes (including MnSOD) in preparation for reoxygenation. Smad and STAT transcription factors, following the pattern of pro-development Akt signaling, also showed partially active profiles (via phosphorylation status) but up-regulation was not uniform. Hepatopancreas showed a more active profile of Smads, but this did not correlate with increased DNA binding, again hinting at a preparative mechanism for the recovery period. Apoptosis (cell death) signaling was assessed through pro-apoptosis (p53) and anti-apoptosis (Bcl) targets, whereas autophagy (a cell minimization response to stress) was assessed via expression response of multiple autophagy proteins (Atg). An anoxia-triggered, tissue-specific result arose, potentially based on the importance of individual organ integrity throughout hypometabolism. Tail muscle, which showed increased expression profiles of all three 
target groups (p53, Bcls, Atgs), contrasted with hepatopancreas, which appeared to be not susceptible to either apoptotic or autophagic signaling during anoxia. Finally, the cell cycle, often a target for attenuation in stress states, was analyzed. Neither tissue showed strong signs of cell cycle attenuation under anoxia, although certain inhibitor profiles were enhanced under anoxia. The data provide a comprehensive overview of the responses and integration of multiple stress-responsive signaling pathways in $O$. virilis that provide a novel contribution to our understanding of pro-survival mechanisms supporting invertebrate anoxia resistance. 


\section{Acknowledgements}

As I am writing this, I realize that I have spent the last 6 years, in some form or another, in the Storey Lab. A period just under a quarter of my life! Being in this lab has shaped my outlook on the scientific world; something that I was not altogether convinced was right for me throughout large parts of my undergraduate degree. As Ken likes to tell people, I was rescued from the woods (literally and figuratively), and but for my honours thesis experience, I could find myself now in some wretched hive of scum and villainy in Northern Ontario, with only the prospect of planting trees to occupy my mind. The persistence of plaid in my wardrobe suggests that I am still of two minds.

I would like to thank Dr. Kenneth Storey for taking me and my scientific career by the scruff of the neck and making me into something competent and useful, though the jury may still be out on the latter. He has been, and will continue to be, a scientific role model throughout my career, and I am immensely thankful for my time under his tutelage. Of course, I must also thank Jan Storey. She is the anchor of the lab. Her expansive knowledge and continuous editing keep the lab running. Many times she has had to endure my idiocy, but has done so with immense patience.

Unfortunately, I have been in the lab too long to list and thank all of my fellow lab members, past and present, but I would like to note that one of the most important qualities of the Storey lab is its incredible sense of community. It is a lab built on shared knowledge, and is all the more successful for it. I can only hope my future labs are as kind and supportive. 
Finally, and most importantly, I would like to thank my family. My enthusiasms can be fitful, so I have always been grateful for their constant and unwavering support throughout my life. 


\section{TABLE OF CONTENTS}

Title Page

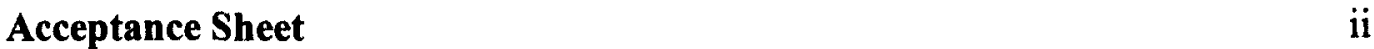

Abstract

Acknowledgements $\quad \mathrm{V}$

Table of Contents vii

List of Abbreviations

List of Figures $\quad$ xi

List of Tables $\quad$ xvi

List of Appendices xvii

Chapter 1 General Introduction 1

Chapter 2 Regulation of Akt signaling, and its effect on the FoxO 21 transcription factors during anoxia in the freshwater crayfish

Chapter 3 Smad and STAT signaling in freshwater crayfish;

Regulation of developmental pathways under anoxia

Chapter 4 Activation of the tumor suppressor protein, p53 in the 126 anoxia resistant crayfish

$\begin{array}{lll}\text { Chapter } 5 \text { Regulation of apoptosis and autophagy during anoxia in the } & 161\end{array}$ freshwater crayfish $O$. virilis

Chapter 6 Regulation of the cell cycle during oxygen deprivation by 194 the anoxia resistant crayfish, $O$. virilis

$\begin{array}{lll}\text { Chapter } 7 \text { General Discussion } & 240\end{array}$

$\begin{array}{ll}\text { Appendices } & 259\end{array}$

$\begin{array}{ll}\text { References } & 269\end{array}$ 


\section{LIST OF ABBREVIATIONS}

AA

4EBP

AK

AMP, ADP, ATP

AMPK

AMPK

APC/C

APS

Atg

ATM

ATR

Bcl

BH3

BLAST

BMP

Bp

CAK

cAMP

Cdc

CDH1

CDK

cDNA

Cep-1

CHK

Cip

Cu/Zn SOD

Cvt

DAF

$\operatorname{ddH}_{2} \mathrm{O}$

DEPC

dNTP

DRAM

DTT

E2F

ECL

EDTA

eEF2 (K)

Ei24 amino acid

eIF4E binding protein

Arginine kinase

adenosine mono-, di-, or triphosphate

AMP activated protein kinase

AMP activated protein kinase

Anaphase promoting complex/cyclosome

ammonium persulphate

Autophagy gene

Ataxia telangiectasia mutated

ATM-and Rad3 related

B-cell lymphoma

Bcl-2 homology domain

Basic Local Alignment Search Tool

Bone morphogenic protein

Base pairs

CDK-activating kinase

3', 5'-cyclic adenosine monophosphate

Cell division cycle protein

Cadherin-1

cyclin dependent kinase

complementary deoxyribonucleic acid

C. elegans $\mathrm{p} 53$

Checkpoint kinase

Cyclin inhibiting protein

Copper/Zinc superoxide dismutase

Cytoplasm-to-vacuole targeting

Abnormal dauer formation

Double distilled water

diethylpyrocarbonate

deoxynucleotide triphosphate

Damage regulated autophagy modulator

dithiolthreitol

E2 promoter binding factor

enhanced chemiluminescence

ethylenediamine tetraacetic acid

Eukaryotic elongation factor 2 (kinase)

Etoposide induced 24 


\begin{tabular}{|c|c|}
\hline eIF2a & eukaryotic initiation factor $2 \alpha$ subunit \\
\hline elF4E & Eukaryotic translation initiation factor $4 \mathrm{E}$ \\
\hline ELISA & enzyme-linked immunosorbent assay \\
\hline EMSA & Electromobility shift assay \\
\hline ERK & Extracellular signal regulated kinases \\
\hline EtBr & ethidium bromide \\
\hline ETC & electron transport chain \\
\hline F6P & Fructose-6-phosphate \\
\hline FoxO & forkhead box, subclass $O$ protein \\
\hline $\mathbf{G}_{\mathbf{1}}$ & Gap phase 1 \\
\hline $\mathbf{G}_{\mathbf{2}}$ & Gap phase 2 \\
\hline G6P & Glucose-6-phosphate \\
\hline GDF & Growth and differentiation factor \\
\hline $\mathbf{G}_{\mathbf{0}}$ & Quiescence \\
\hline GSK-3 & Glycogen synthase kinase- 3 \\
\hline GßL & G-protein subunit \\
\hline HEPES & $\begin{array}{l}\text { N-(2-hydroxyethyl) piperazine-N'- (2-ethanesulfonic } \\
\text { acid) }\end{array}$ \\
\hline HIF-1 & hypoxia-inducible factor-1 \\
\hline HRP & horseradish peroxidase \\
\hline $\mathbf{I} / \mathbf{R}$ & Ischemia/Reperfusion \\
\hline IFN & Interferon \\
\hline IGF & Insulin like growth factor \\
\hline IGF-IR & Insulin-like growth factor receptor \\
\hline IkB & inhibitor of NFkB \\
\hline INK4 & Inhibitor of CDK4 \\
\hline IR & Ionizing radiation \\
\hline JAK & Janus kinase \\
\hline JNK & jun-N-terminal kinase \\
\hline $\mathbf{K A P}$ & Kinase associated phosphatase \\
\hline kb & kilobase \\
\hline $\mathbf{k D a}$ & kilodalton \\
\hline Kip & Kinase inhibiting protein \\
\hline $\mathbf{M}$ & Mitosis \\
\hline MAPK & Mitogen activated protein kinase \\
\hline MDM2 & Mouse double minute 2 \\
\hline MHC & Myosin heavy chain \\
\hline MnSOD & manganese superoxide dismutase \\
\hline MPF & Maturation promoting factors \\
\hline MRD & Metabolic rate depression \\
\hline
\end{tabular}


mRNA

Myc

$\mathrm{Na}^{+} \mathbf{K}^{+}$-ATPase

NCBI

NF-kB

NLS

OMM (P)

ORF

P19 ARF

p70S6K

PAGE

PBS

PCNA

PCR

PDK1

PE

PFK

PI3K

PIAS

PIP2

PIP3

PKA

PKC

PMSF

PP1

PP2A

PP2C

PRAS40

PTEN

PTM

PVDF

Rb

ROS

RT

RT-PCR

S

SARA

SDS

SGK messenger RNA

Myelocytomatosis

ATP-dependent sodium potassium pump

National Center for Biotechnology Information

nuclear factor kappa $B$

Nuclear localization sequence

Outer mitochondrial membrane (pore)

open reading frame

P19 Alternate reading frame

p70 S6 protein kinase

polyacrylamide gel electrophoresis

phosphate-buffered saline

Proliferating cell nuclear antigen

polymerase chain reaction

phosphoinositide-dependent kinase

Phosphatidyl ethanolamine

6-phosphofructokinase

phosphoinositide 3-kinase

Protein inhibitors of STATs

phosphatidylinositol 4,5-bisphosphate

phosphatidyl inositol-(3,4,5)-triphosphate

cAMP- activated protein kinase

Protein kinase $\mathrm{C}$

phenylmethanesulfonyl fluoride

Protein phosphatase 1

protein phosphatase $2 \mathrm{~A}$

Protein phosphatase $2 \mathrm{C}$

$40 \mathrm{kDa}$ proline-rich Akt1 substrate

phosphatase and tensin homologue deleted on chromosome

10

Post-translational modification

polyvinylidine fluoride

retinoblastoma protein

reactive oxygen species

room temperature $\left(-21^{\circ} \mathrm{C}\right)$

reverse-transcriptase polymerase chain reaction

DNA synthesis/replication

Smad anchor for receptor activation

sodium dodecyl sulfate

Serum glucocorticoid kinase 
Smad

SOCs

STAT

TAE

TBST

TEMED

TGF- $\beta$

TMB

TOR

Tris

TSC1/2

UDP

UPR

Vps34
Mothers against decapentaplegic

Suppressors of cytokine signaling signal transducer and activator of transcription tris-acetate-ethylenediamine tetraacetic acid buffer tris-buffered saline containing Tween-20 $\mathrm{N}, \mathrm{N}, \mathrm{N}$ ',N'-tetra methylethylenediamine Transforming growth factor $\beta$ 3,3',5,5'-Tetramethylbenzidine Target of rapamycin ( $\mathrm{m}$ - mammalian) tris (hydroxymethyl) aminomethane Tuberous sclerosis proteins 1 and 2 Uridine diphosphate unfolded protein response

Vacuolar protein sorting- associated protein 34

\section{LIST OF FIGURES}

Figure 1.1 The freshwater crayfish, Orconectes virilis.

Figure 1.2 Hypoxic coastal regions and their effects on marine biodiversity.

Figure 1.3 The life cycle of the nematode, C. elegans.

Figure 2.1 An overview of the Akt pathway.

Figure 2.2 Determination of optimum PCR cycle count by analysis of signal saturation at multiple concentrations.

Figure 2.3 Tubulin concentration gradient showing measurable signal with increasing dilution.

Figure 2.4 Partial nucleotide and deduced amino acid sequence of $O$. virilis alpha tubulin II protein.

Figure 2.5 Partial nucleotide and deduced amino acid sequence of $O$. virilis myosin heavy chain protein.

Figure 2.6 Effect of $20 \mathrm{~h}$ anoxia exposure on Akt protein levels and its phosphorylation (at $\mathrm{Thr} 308$ ) in $O$. virilis tissues. 
Figure 2.7 Effect of $20 \mathrm{~h}$ anoxia exposure on nuclear expression of Akt 62 phosphorylation (T308) in $O$. virilis tissues.

Figure 2.8 Effect of $20 \mathrm{~h}$ anoxia exposure on phosphorylation of PDK1 (S241) 63 in $O$. virilis tissues.

Figure 2.9 Effect of $20 \mathrm{~h}$ anoxia exposure on the subcellular distribution of $\quad 64$ PTEN in $O$. virilis tissues.

Figure 2.10 Effect of $20 \mathrm{~h}$ anoxia exposure on GSK3a protein levels and activation in $O$. virilis tissues.

Figure 2.11 Effect of $20 \mathrm{~h}$ anoxia exposure on SGK protein levels and activation 66 in $O$. virilis tissues.

Figure 2.12 Effect of $20 \mathrm{~h}$ anoxia exposure on FoxOl and $\mathrm{FoxO} 3$ protein levels in $O$. virilis tissues.

Figure 2.13 Effect of $20 \mathrm{~h}$ anoxia exposure on the subcellular distribution of FoxO1 in $O$. virilis tissues.

Figure 2.14 Effect of $20 \mathrm{~h}$ anoxia exposure on the subcellular distribution of FoxO3 in $O$. virilis tissues.

Figure 2.15 Effect of $20 \mathrm{~h}$ anoxia exposure on phosphorylation of FoxOl in $O$. 70 virilis tissues.

Figure 2.16 Effect of $20 \mathrm{~h}$ anoxia exposure on localization of phosphorylation of 71 FoxO1 in $O$. virilis tissues.

Figure 2.17 Effect of $20 \mathrm{~h}$ anoxia exposure on FoxO (1 and 3) DNA binding activity in $O$. virilis tissues.

Figure 2.18 Partial nucleotide and deduced amino acid sequence of $O$. virilis MnSOD-like protein.

Figure 2.19 Effect of $20 \mathrm{~h}$ anoxic exposure on $M n S O D$-like transcript expression in $O$. virilis tissues as determined by RT-PCR.

Figure 2.20 Effect of $20 \mathrm{~h}$ anoxia exposure on MnSOD expression in $O$. virilis 75 tissues.

Figure 2.21 Effect of $20 \mathrm{~h}$ anoxia exposure on catalase expression in $O$. virilis $\quad 76$ tissues.

Figure 2.22 Effect of $20 \mathrm{~h}$ anoxia exposure on nuclear 14-3-3 expression in $O$. virilis tissues. 
Figure 3.1 An overview of the TGF- $\beta$ pathway.

Figure 3.2 A potential mechanism for interaction between the prodevelopmental TGF- $\beta$ and JAK-STAT pathways.

Figure 3.3 Effect of $20 \mathrm{~h}$ anoxic exposure on Smad 1/5-p activation in $O$. virilis 108 tissues.

Figure 3.4 Effect of $20 \mathrm{~h}$ anoxic exposure on Smad 2-p activation in O.virilis tissues.

Figure 3.5 Effect of $20 \mathrm{~h}$ anoxic exposure on Smad 2-p localization in O.virilis 110 tissues.

Figure 3.6 Partial nucleotide and deduced amino acid sequence of $O$. virilis Smad 3-like protein.

Figure3.7 Effect of $20 \mathrm{~h}$ anoxic exposure on Smad 3-like transcript expression in $O$. virilis tissues as determined by RT-PCR.

Figure 3.8 Effect of $20 \mathrm{~h}$ anoxic exposure on Smad 3 levels in O.virilis tissues. $\quad 114$

Figure 3.9 Effect of $20 \mathrm{~h}$ anoxic submergence on Smad 3 activity in $O$. virilis tissues.

Figure 3.10 Effect of $20 \mathrm{~h}$ anoxic exposure on Smad 4 levels in O.virilis tissues. 116

Figure 3.11 Partial nucleotide and deduced amino acid sequence of $O$. virilis Stat 1-like protein.

Figure 3.12 Effect of $20 \mathrm{~h}$ anoxic exposure on Stat-1 -like transcript expression in $O$. virilis tissues as determined by RT-PCR.

Figure 3.13 Effect of $20 \mathrm{~h}$ anoxic exposure on Stat 1-p activation in O.virilis 120 tissues.

Figure 3.14 Effect of $20 \mathrm{~h}$ anoxic submergence on Stat 1 activity in $O$. virilis tissues.

Figure 3.15 Effect of $20 \mathrm{~h}$ anoxic exposure on Stat 2-p activation in O.virilis tissues.

Figure 3.16 Effect of $20 \mathrm{~h}$ anoxic exposure on Stat 3-p activation in O.virilis 123 tissues. 
Figure 3.17 Effect of $20 \mathrm{~h}$ anoxic exposure on Stat 5 and 6 protein levels in O.virilis tissues.

Figure 4.1 An overview of the p53 DNA damage response.

Figure 4.2 Effect of $20 \mathrm{~h}$ anoxic exposure on ATM and ATR activation in $O$. virilis tissues.

Figure 4.3 Effect of $20 \mathrm{~h}$ anoxic exposure on $\mathrm{p} 53$ protein levels in $O$. virilis 150 tissues.

Figure 4.4 Effect of $20 \mathrm{~h}$ anoxic exposure on p53-p (Ser 15) activation in $O$. virilis tissues.

Figure 4.5 Effect of $20 \mathrm{~h}$ anoxic exposure on the localization of phosphorylated 152 p53 in $O$. virilis tissues.

Figure 4.6 Effect of $20 \mathrm{~h}$ anoxia exposure on p53 DNA-binding activity in $O$. virilis tissues.

Figure 4.7 Partial nucleotide and deduced amino acid sequence of $O$. virilis ei24-like protein.

Figure 4.8 Effect of $20 \mathrm{~h}$ anoxic exposure on ei24-like transcript expression in $\quad 156$ $O$. virilis tissues as determined by RT-PCR.

Figure 4.9 Effect of $20 \mathrm{~h}$ anoxia exposure on $\mathrm{p} 53-\mathrm{p}$ (Ser 37) activation in $O$. 157 virilis tissues.

Figure 4.10 Effect of $20 \mathrm{~h}$ anoxic exposure on MDM2 protein levels in $O$. virilis tissues.

158

Figure 4.11 Effect of $20 \mathrm{~h}$ anoxic exposure on p19 ARF protein levels in $O$. virilis tissues.

Figure 5.1 An overview of apoptosis and autophagy regulation.

Figure 5.2 Regulation of autophagy by TORC1.

Figure 5.3 Effect of $20 \mathrm{~h}$ anoxic exposure on the Bcl2 protein levels and activation in $O$. virilis tissues.

Figure 5.4 Effect of $20 \mathrm{~h}$ anoxic exposure on $\mathrm{Bcl} 3$ and $\mathrm{Bcl}-\mathrm{xl}$ protein levels in 185 $O$. virilis tissues. 
Figure 5.5 Effect of $20 \mathrm{~h}$ anoxic exposure on Beclin 1 expression in O. virilis tissues.

Figure 5.6 Effect of $20 \mathrm{~h}$ anoxic exposure on Atg5 and 12 protein levels in $O$. virilis tissues.

Figure 5.7 Effect of $20 \mathrm{~h}$ anoxic exposure on Atg7 expression in O. virilis tissues.

Figure 5.8 Effect of $20 \mathrm{~h}$ anoxic exposure on Atg8 protein levels and activation $\quad 189$ in $O$. virilis tissues.

Figure 5.9 Effect of $20 \mathrm{~h}$ anoxic exposure on mTOR expression and TORC 190 incorporation in $O$. virilis tissues.

Figure 5.10 Effect of $20 \mathrm{~h}$ anoxic exposure on G $\beta \mathrm{L}$ expression and TORC incorporation in $O$. virilis tissues.

Figure 5.11 Effect of $20 \mathrm{~h}$ anoxic exposure on phosphorylation of PRAS40 and p70S6K in O.virilis tissues.

Figure 6.1 An overview of the cell cycle.

Figure 6.2 Effect of $20 \mathrm{~h}$ anoxia exposure on protein expression of CDK2 and 222 4 in $O$. virilis tissues.

Figure 6.3 Effect of $20 \mathrm{~h}$ anoxia exposure on the protein expression and subcellular distribution of CDK6 in $O$. virilis tissues.

Figure 6.4 Effect of $20 \mathrm{~h}$ anoxia exposure on the subcellular distribution of 224 Cyclin $\mathrm{D}$ in $O$. virilis tissues.

Figure 6.5 Effect of $20 \mathrm{~h}$ anoxia exposure on the subcellular distribution of 225 Cyclin $\mathrm{E}$ in $O$. virilis tissues.

Figure 6.6 Effect of $20 \mathrm{~h}$ anoxia exposure on the subcellular distribution of Cyclin $\mathrm{A}$ in $O$. virilis tissues.

Figure 6.7 Effect of $20 \mathrm{~h}$ anoxia exposure on the subcellular distribution of Cyclin $\mathrm{B}$ in $O$. virilis tissues.

Figure 6.8 Partial nucleotide and deduced amino acid sequence of $O$. virilis $\mathrm{Rb}-228$ like protein.

Figure 6.9 Effect of $20 \mathrm{~h}$ anoxic exposure on $R b$-like transcript expression in 229 $O$. virilis tissues as determined by RT-PCR. 
Figure 6.10 Effect of $20 \mathrm{~h}$ anoxia exposure on phosphorylation of $\mathrm{Rb}(\mathrm{S} 780)$ in 230 O. virilis tissues.

Figure 6.11 Effect of $20 \mathrm{~h}$ anoxia exposure on protein expression of E2F-4 in $O . \quad 231$ virilis tissues.

Figure 6.12 Effect of $20 \mathrm{~h}$ anoxia exposure on the protein expression and subcellular distribution of $\mathrm{E} 2 \mathrm{~F}-1$ in $O$. virilis tissues.

Figure 6.13 Effect of $20 \mathrm{~h}$ anoxia exposure on E2F (1 and 4) DNA binding 233 activity in $O$. virilis tissues.

Figure 6.14 Effect of $20 \mathrm{~h}$ anoxia exposure on activation of Chk1 and 2 in $O$. 234 virilis tissues.

Figure 6.15 Effect of $20 \mathrm{~h}$ anoxia exposure on nuclear protein expression of cdc25C in $O$. virilis tissues.

Figure 6.16 Effect of $20 \mathrm{~h}$ anoxia exposure on p15/16 protein levels and pl6 236 activation in $O$. virilis tissues.

Figure 6.17 Effect of $20 \mathrm{~h}$ anoxia exposure on phospho-control and subcellular distribution of $\mathrm{p} 21-\mathrm{p}$ (T145) in $O$. virilis tissues.

237

Figure 6.18 Effect of $20 \mathrm{~h}$ anoxia exposure on protein expression of $\mathrm{p} 27$ in $O$. virilis tissues.

\section{LIST OF TABLES}

Table 2.1 List of Primers used in Chapter $2 \quad 35$

Table 2.2 List of primary antibodies used in Chapter 2

$\begin{array}{lll}\text { Table 2.3 Chapter } 2 \text { data summary } & 78\end{array}$

$\begin{array}{lll}\text { Table 3.1 List of Primers used in Chapter } 3 & 88\end{array}$

Table 3.2 List of primary antibodies used in Chapter $3 \quad 88$

$\begin{array}{lll}\text { Table 3.3 Chapter } 3 \text { data summary } & 125\end{array}$

Table 4.1 List of Primers used in Chapter $4 \quad 134$

$\begin{array}{lll}\text { Table 4.2 List of primary antibodies used in Chapter } 4 & 134\end{array}$

$\begin{array}{lll}\text { Table 4.3 Chapter } 4 \text { data summary } & 160\end{array}$ 
Table 5.1 List of primary antibodies used in Chapter 5

Table 5.2 Chapter 5 data summary

Table 6.1 List of Primers used in Chapter 6

Table 6.2 List of primary antibodies used in Chapter 6

Table 6.3 Chapter 6 data summary

\section{LIST OF APPENDICES}

$\begin{array}{lll}\text { Appendix A } & \text { Trends in C. elegans dauer response } & 260\end{array}$

$\begin{array}{lll}\text { Appendix B } & \text { C. elegans descriptions } & 261\end{array}$

$\begin{array}{lll}\text { Appendix C List of publications } & 266\end{array}$

$\begin{array}{lll}\text { Appendix D } & \text { Communications at scientific meetings } & 267\end{array}$

Appendix E Ensuring antibody specificity for western blotting 268 


\section{Chapter 1 General Introduction}

In this small fish I take it that human wisdom is admirably figured and symbolized; for whereas the crayfish doth move only backward, and can have only retrospection, seeing naught but the perils already passed, so the wisdom of man doth not enable him to avoid the follies that beset his course, but only to apprehend their nature afterward.

- Sir James Merivale 
Metabolic rate depression

Stress, and the concept of stress adaptation as pioneered by Hans Selye, is the interplay between damage and defense (Selye, 1950). Selye established the concept of a general adaptation to stress, regardless of stressor. Within this concept, three stages were characterized as 'alarm reaction', 'stage of resistance' and the 'stage of exhaustion' (Selye, 1950). While this theory was originally established in the context of endocrine and neuronal responses to a generic stressor, along with the concept of homeostasis by Claude Bernard and Walter Cannon, this formed the basis of cellular adaptations to stress through prolonged periods of exposure. This research opened the thought of a controlled and generic stress response, regardless of the model or the stressor. Guppy and Withers summarized the breadth of the field in 1999, elucidating the capacity for metabolic rate depression in the majority of animal phyla. While a normal range of metabolic rate depression, in response to adverse environmental conditions, was seen to be between 60 to $80 \%$, there are many cases of up to $99 \%$ suppression in relation to normal resting metabolic rates, and even further (up to $100 \%$ ) in non-cryptobiotic (some mollusc, crustacean, fish, amphibian, reptile and worm systems) animals (Guppy and Withers, 1999). As stress response studies advanced, the roles of physiological adaptations and stress responsive molecular pathways on cellular metabolism were pushed to the forefront; specifically, the works of Hochachka, Lutz and Storey have centered on the depression of cellular metabolism in the efforts to maintain cellular homeostasis and survival. 
An organism's metabolism is a dynamic process that adapts in response to internal and external stressors. Many organisms encounter environmental stress in their natural habitats, brought on by acute or seasonal temperature changes, changes in water availability and/or salinity, reduced oxygen levels (hypoxia/anoxia) or a drop in nutrient availability (caloric restriction), for example. Adaptations begin through sensory processing, either directly sensing the environmental parameter or, in the case of seasonal stress, often triggered by changes in photoperiod. Responses by animals can be compensatory to allow life to continue normally or preservation responses may be initiated that minimize fuel and energy use and put in place adaptations to sustain life until the stress is lifted. Responses are often made first at the physiological level; in the case of preservation responses these can include a strong reduction in energy expenditure on movement, altered respiration patterns, abated digestion, etc. Prolonged environmental stress, particularly when oxygen levels or delivery are affected (consequently disrupting aerobic metabolism), affects the energy state of the cell. At the molecular level, the cells must balance the energetic expenses with prolonged viability. This prompts either prosurvival or pro-death (e.g. apoptosis) signals. Two major pro-survival responses are metabolic rate depression (MRD; a strong suppression of energetically costly processes in cells so that net energy expenditures are greatly reduced) and autophagy (a regulated catabolism of selected subcellular components). Both are options for animals that are well-adapted survive prolonged environmental stress. Autophagy as an adaptive strategy for dealing with environmental stress has actually received very little attention to date but MRD has been well studied for many years. 
Suppression of metabolic rate, as mentioned above, occurs widely across phylogeny, as a method of maintaining energetic homeostasis while under stress conditions. This suppression allows an inverse elevation of the time that organisms can survive using only endogenous fuel reserves. There are many types of hypometabolism, characterized by this drop in metabolic rates, including hibernation in mammals (Storey and Storey, 2010a), estivation in land snails (Storey and Storey, 2010b), cold hardiness in wood frogs (Storey et al., 1996) and diapauses in insects (Denlinger, 2002). Survival throughout the period of hypometabolism is managed via a reprioritization of fuel use, a redistribution of energy to core processes, a reduction of energy-expensive processes (ion channel regulation, certain protein synthesis, cell growth, development and division) and the activation of protective mechanisms (elevation of antioxidants, anti-freeze proteins, molecular chaperones, etc.) (Storey and Storey, 2004).

\section{Oxygen deprivation}

Potentially the most critical aspect of many of forms of environmental stress is that oxygen availability can be restricted or lost. Environmental limitations of oxygen (ex. ice cover on bodies of water), along with physiological responses (ex. apnoic breathing) or severely reduced circulation (e.g. freezing induced ischemia) are all mechanisms that can lead to hypoxia or anoxia. Modifications to aerobic respiration to improve oxygen uptake and delivery are often the first response to oxygen limitation but ultimately adjustments that enhance anaerobic capacity are typically also needed. The capacity for organisms to use oxygen in metabolism, developed over two billion years ago, has allowed a rapid increase in organismal complexity, due to the ability to extract greater amounts of energy from organic molecules. As the final electron carrier in the 
electron chain, oxygen deprivation critically affects the production of ATP and subsequently the rates of the multitude of cellular processes that are driven by ATP. Oxygen levels are monitored physiologically, often by specific oxygen sensors, and on the molecular level typically by heme proteins (Hochachka and Lutz, 2001); changes in oxygen availability rapidly alter the rates of both existing enzymes and functional proteins and also change gene expression and the suite of new proteins that can be made. In terms of metabolic rate depression, animals that are anoxia tolerant often see a reduction of up to $99.9 \%$ of their resting metabolism; even animals that are only anoxia resistant see drops of up to $99.5 \%$ of their metabolic rate (Guppy and Withers, 1999). As one of the most energetically expensive procedures in the cell, ATP dependent ion channels (which consume upwards of $30 \%$ of total cellular ATP), are strongly modified during MRD, resulting in a coordinated suppression of both ion motive ATPases and ATP-independent ion pumps such that membrane potential difference can be sustained under hypoxia/anoxia (Hochachka and Lutz, 2001). In anoxia/hypoxia intolerant species the inability to maintain membrane potential difference when oxygen levels fall is a primary reason for injury or death under these conditions. The loss of membrane potential difference leads to major changes in ionic concentrations; particularly greatly increased intracellular $\mathrm{Ca}^{2+}$. Amongst other things, elevated intracellular $\mathrm{Ca}^{2+}$ causes increased protein kinase signaling (PKA/PKC; Roberson et al., 2010), and stimulation of many proteases, calpains and excitatory responses that can lead to cell death (Kreiger and Duchen, 2002). Hence, hypoxia/anoxia resistant animals must mediate the increased calcium levels, including the transport of cytosolic $\mathrm{Ca}^{2+}$ back into the ER where it is typically stored, through the modifications to the SERCA pump. 
Another major process regulated in response to energetic limitation is protein synthesis. Protein synthesis is first reduced specifically in the hepatocytes in response to early hypoxia sensor signals, and secondly throughout the organism via translational arrest (Hochachka and Lutz, 2001). Further to these mechanisms, altered fuel . metabolism, carbon flux and protein refolding/degradation decisions are made in response to the altered energy state (Storey and Storey, 2004), and consequently require a complex and variable method of regulation.

Regulation in MRD:

As such, regulating the suppression of metabolism are numerous mechanisms that target transcriptional, translational and post-translational levels of gene expression. While transcriptional controls vary from histone modification, the action of microRNAs (Biggar and Storey, 2011) and modification of transcription factor activities, translational control can be manifested through the sequestration of target mRNAs into monosomes and ribonuclear protein complexes (storage granules) throughout the hypometabolic period (Storey and Storey, 2004). Possibly the most prominent and abundant level of regulation in response to stress conditions, occurs at the posttranslational level; the adaptation of protein activity through physical modification. Posttranslational modifications (PTM), conferred by one protein onto another target protein are able to regulate rapid signal transduction as well as modify the activity states of many functional proteins to allow transition into a hypometabolic state. Amongst the most common PTMs are reversible phosphorylation, acetylation, sumoylation, methylation and ubiquitination. Of these mechanisms, perhaps the most important is reversible protein phosphorylation, and the key role of this mechanism in is widely implicated in both vertebrate and invertebrate 
models of MRD (MacDonald and Storey, 1999; McMullen and Storey, 2008). Protein kinases and protein phosphatases, the effectors of reversible phosphorylation, are abundant in cells and are critical in signal transduction from cell membrane receptors in response to many different stressors. Protein kinases typically target serine/threonine residues or tyrosine residues, with phosphatases having similar divisions. Major protein kinases are implicated in stress responsive regulation of cellular processes from ion pump regulation (MacDonald and Storey, 1999) to the cell cycle (Reinhardt and Yaffe, 2009). The anoxia resistant crayfish, Orconectes virilis

The virile crayfish, Orconectes virilis, is a freshwater crustacean native to North America (FIG. 1.1). In Canada, it is prominent in Southern Ontario, but is located across the country. In parts of North America, it is an invasive species (Aiken, 1965). These crayfish can be found in rock-pools, river/streambeds and lakeshores, and have the capacity to venture into deep water (maximum depth $\sim 30$ feet); they are often the only species of crayfish in a given body of water (Crocker and Barr, 1968). The typical lifespan for $O$. virilis is 3 years, which includes a maximum of two mating seasons (Crocker and Barr, 1968). In terms of their ecological presence, being omnivorous and scavengers, they are able to find nutrition in almost every habitat. They have a preference for decaying material and consume a range of foodstuffs from vegetation to other aquatic invertebrates (Crocker and Barr, 1968). Conversely, they are highly preyed upon by a range of animals including fish, amphibians and birds. Species from the Orconectes genus are typically too small to be farmed, and as such are not typically considered to be commercially important beyond their use as fishing bait (Holdich and Lowery, 1988). This means that very little research has been conducted in relation to their use in industry. 
Even so, the majority of crayfish research focuses on commercial aspects including host immunity (the prevalent white spot syndrome virus; Oidtmann and Stentiford, 2011) and moulting cycles (maturity; Nakatsuji et al., 2009). Hence, research on crayfish resistance and adaptation to environmental stressors is fairly limited, and furthermore, most available studies are largely focused on physiological responses. Nonetheless, this research forms the groundwork for biochemical studies of stress responses. Interestingly, in the near future, data on stress responses (particularly to oxygen deprivation) in crustaceans may become increasingly important as coastlines become increasingly hypoxic; these are, traditionally, areas that are fished for many crustacean species. Vaquer-Sunyer and Duarte (2008) recently appraised the rapid increase in hypoxic coastal sites (FIG 1.2i) around the world, and its effects on marine biodiversity, illustrating the differing abilities of marine life to adapt to reduced oxygen levels. With water temperature inversely proportionate to oxygen solubility, global warming and the resultant increased water temperatures have created increased hypoxic zones. The inherent capacity for oxygen deprivation, amongst the taxa that comprise marine biodiversity, is based largely upon the capacity to migrate (FIG 1.2ii) (Vaquer-Sunyer and Duarte, 2008). Fish and crustaceans have the lowest innate ability to survive hypoxia, and it has begun to affect their industrial value, as well as their ecological prevalence in certain regions.

The freshwater crayfish, $O$. virilis, can be challenged by environmental oxygen deprivation in both summer and winter seasons. In the summer, crayfish may be isolated in low flow, high temperature, low $\mathrm{O}_{2}$ content streambeds, whereas in winter, the animals are often trapped in ice covered waters where oxygen is depleted by 
animal/plant/microorganism respiration. During the winter months (January through to March ice breakup), oxygen drops to $0 \mathrm{ppm}$ (F.W. Schueler, personal communication), and the crayfish must display anoxia resistance to overcome this metabolic challenge. In the winter months, the crayfish also have to contend with extremely low $\left(0-4^{\circ} \mathrm{C}\right)$ temperatures and the possibility of winterkill by freezing. Indeed, when $O$. virilis winter acclimatization was tested, and it was found that the crayfish cannot survive even low exposure to freezing, attempting instead to move to deeper, unfrozen water (also linked with maturation stage) or rocky water that does not freeze to the bottom (Aiken, 1967).

A number of hypoxia tolerant members within the crustacean taxa have been identified, and studies of their physiological adaptations have centered on respiratory and circulatory adaptations. Hyperventilation upon sensing reduced oxygen is common, and the resulting alkalosis triggers a change in oxygen binding affinity of the crustacean oxygen binding/transporting protein, hemocyanin (McMahon, 2001). Found in the hemolymph of most decapod crustaceans, hemocyanin is a highly plastic, multi-subunit protein that assembles into hexameric complexes which adapt to the low $\mathrm{O}_{2}$ levels with increased affinity; as the crustacean shifts into anaerobic metabolism, the buildup of lactate further increases hemocyanin $\mathrm{O}_{2}$ affinity (McMahon, 2001). The cardiac output of the crayfish may also be altered in response to hypoxia. Bradycardia, or slowing of the heart beat, was observed in conjunction with increased stroke volume, allowing reduced muscular contractions and reducing ATP expenditure while maintaining overall cardiac output (Reiber, 1995). In addition, there is a shunt of hemolymph to the anterior end of the crayfish (Reiber, 1995) in order to preserve oxygen availability to the nervous tissue, and presumably allow for continued stress response signaling despite oxygen limitation. 
Anaerobic metabolism in crayfish is based on glycolysis, with lactate accumulating with some contribution from the phosphagen, arginine phosphate, primarily in muscle tissues. The energetic change from aerobic to anaerobic metabolism has the effect of altering ion concentrations in cells, with not only the acidification associated with lactate metabolism releasing protons, but increased cytosolic $\mathrm{Ca}^{2+}$ levels that are deleterious to the cell. In many cases, hypoxia tolerant crustaceans are able to ameliorate these changes in ionic concentration through carapace mediated buffering. Both through sequestering of lactate in the carapace (potentially bound to calcium ions), and mobilization of carbonate ions ( $\mathrm{HCO}^{-}$from calcium carbonate) to buffer against cations (including protons), the crayfish is able to prevent cellular acidification (Jackson et al,, 2001). During recovery after hypoxia/anoxia, the priority is to restore energy state. Amongst the earliest targets for replenishment (seen in hypoxia recovery crayfish) are arginine phosphate and free glucose, while lipids were the primary source of energy during the recovery (da Silva-Castiglioni et al., 2011).

Using the model C. elegans as a template for $O$. virilis

Despite a considerable body of research on crustacean physiological responses to oxygen deprivation, less is known about the metabolic responses to hypoxia/anoxia, especially in $O$. virilis. In unpublished studies from our lab, $O$. virilis showed good anoxia resistance, readily surviving $24 \mathrm{~h}$ in nitrogen-treated freshwater at both $10^{\circ}$ and $15^{\circ} \mathrm{C}$. Anoxia exposure (1 hour and 4 hour) or exercise (which creates a state of functional hypoxia), reduced levels of arginine phosphate significantly in $O$. virilis tail muscle, whereas AMP and ADP levels increased strongly (K.B Storey, data unpublished). Levels of free glucose and L-lactate also rise significantly. Enzymatic data 
showed a rapid 3-fold increase in the amount of active glycogen phosphorylase within $1 \mathrm{~h}$ that gradually fell again to control level as anoxia was prolonged. Two forms of both pyruvate kinase and phosphofructokinase were identified in $O$. virilis tail muscle and hepatopancreas with a shift under anoxia to a greater proportion of a low phosphate, less active enzyme form (Cowan, 1998). The enzyme changes are consistent with the use of carbohydrate fuels under anoxia but indicate that the shift from aerobic to anaerobic metabolism involves regulated changes in glycolytic activity, mediated by reversible protein phosphorylation of key enzymes, that are consistent with anoxia-induced MRD as seen in other anoxia tolerant animal models. Another important enzyme of energy metabolism, arginine kinase, is also regulated by anoxia-responsive reversible phosphorylation in O. virilis muscle (Dawson and Storey, 2011). Regulation of crayfish metabolism via reversible phosphorylation of selected enzyme targets during the aerobicanaerobic transition is also supported by significant changes in protein kinase (PKA) and protein phosphatase (PKA/ PP1/2A/2C) activities in response to anoxia (Cowan and Storey, 2001). Hence, in terms of central energy metabolism there is good evidence for a regulated transition into the anoxia resistant state involving enzyme control via reversible protein phosphorylation coupled with an adapted cellular metabolism to reduce overall energetic needs, similar to patterns established for other hypoxia/anoxia-tolerant species. However, in the crustacean model there is little or nothing known about the responses to anoxia by many other stress responsive pathways and their effects on many other cell functions.

In order to map the key stress responses that confer anoxia resistance by crayfish, a highly researched animal model could provide a template to explore the control of 
anoxia resistance and metabolic adaptation in $O$. virilis. The nematode, Caenorhabditis elegans is a very well studied model organism. Over its own lifespan it can encounter multiple stressors associated with a growing, but not particularly mobile, population; limited nutrient availability, changes in temperature, and decreased oxygen, amongst others (See appendix A and B.1). During this period, at the cellular level, there are many key stress response pathway changes made. These include changes to insulin signaling, development and immunity pathways, cell death/minimization processes and the cell cycle. Thus, triggered by environmental conditions, and transmitted through neuronal and then molecular signals, the nematodes enter an ageless state known as the 'dauer' stage. It is known that the critical element to dauer entry is caloric restriction, and worms faced with this at the L1 larval stage enter the pre-dauer L2d stage, rather than a normal L2 stage, but when conditions are again favourable they exit back to standard development through L3 and L4 into adulthood (FIG 1.3) (Altun and Hall, 2005). Entry into the dauer stage significantly extends the total worm lifespan, and the duration of this period does not affect the post-dauer lifespan (Vanfleteren and Braeckman, 1999). The pathways employed for regulation are molecular pathways that are common through phylogeny, from yeast to mammals, and show high levels of conservation where regulation is concerned (Lant and Storey, 2010). Hence in order to map the critical pathways of stress response of $O$. virilis and provide an overview to anoxia resistance, the mechanisms employed by $C$. elegans in its dauer state were used as outline.

The crayfish is a relatively under studied system, among animal models of anoxia resistance and metabolic rate depression. While the majority of biochemical research to date has been focused on enzymology (specifically, the regulation of target kinases and 
glycolytic enzymes), virtually nothing is known about the role of gene expression in crayfish adaptation to anoxia. 


\section{Objective and Hypotheses}

Acting as a relative blank canvas to this novel invertebrate stress response, this thesis aims to make broad, pathway based assessments of the major stress response pathways that are likely to be involved in MRD triggered by oxygen deprivation. As a template for stress response, critical pathways of model organism $C$. elegans in its dauer stress state were analyzed in the crayfish system. In doing so, this thesis attempts to identify conserved targets (throughout phylogeny) of stress response within known pathways, as well as identify divergent stress responses, potentially exclusive to the freshwater crayfish. This thesis also attempts to discover tissue specific responses by analyzing two different tissues: tail muscle that functions in rapid propulsion of the animal and hepatopancreas, a liver-like organ that conducts a variety of activities including nutrient processing, synthesis of secreted proteins, and detoxification of many chemicals.

This document focuses on three main topics;

1. Regulation of developmental pathways

2. Determination of cellular fate (apoptosis vs. autophagy)

3. Cell cycle control

\section{Regulation of developmental pathways (Chapters 2 and 3)}

The protein kinase B (Akt) signaling pathway, the TGF- $\beta$ and JAK (Janus kinase) signaling pathways are considered to be the most prominent pro-development signaling mechanisms within cells. While the Smads are almost solely development response, the Akt signaling pathway has both energetically expensive and pro-survival downstream 
effects, and within the STATs, STAT 1 is recognized to have pro apoptotic activity while STAT 3 is recognized to have anti-apoptotic activity.

Objectives:

1. Analyze Akt, its upstream activator (PDK1) and downstream targets (GSK3 and FoxO) to obtain a pattern of pathway activity, during anoxia.

2. Analyze Smad and STAT transcription factor activity, during anoxia, through activation patterns and DNA binding levels.

Hypothesis 1: Activation of Akt signaling in response to anoxia will trigger specific pro-survival signals such as anti-apoptotic signaling and antioxidant responses to protect crayfish cells from damage under low oxygen stress.

Hypothesis 2: Enhanced expression and activity of Smad and STAT transcription factors will mediate prosurvival actions under anoxic conditions, particularly via enhancement of anti-apoptotic actions of STATs, concurrent with suppressed pro-apoptotic STAT

\section{Determination of cellular fate (Chapters 4 and 5)}

The p53 tumor suppressor is a major effector in the DNA damage response pathway, and has been identified as a major protein in numerous stress response pathways; even cellular survival pathways. P53 has a canonical pathway of activation and suppression, and affects both mitochondrial membrane stability and cell cycle 
progression to induce autophagy. Preventing outer mitochondrial membrane pore formation are anti-apoptotic members of the $\mathrm{Bcl} 2$ family of proteins ( $\mathrm{Bcl} 2$ and $\mathrm{Bcl} \mathrm{xl})$. Autophagy, a mechanism of recycling cellular components, is an alternative to apoptosis; cell contents are catabolized down to a minimum content that is needed to sustain viability. Autophagy is a commonly observed mechanism in cases of depleted nutrients. A major regulator of autophagy is the TOR kinase, which is a pro-development signal transducer.

Objectives:

1. Analyze $p 53$ signaling under anoxic conditions, via upstream activators (ATM/ATR kinase) and protein level control (activators and inhibitors; p19 ARF and MDM2), along with transcription factor activity levels on a potential downstream target (ei24).

2. Analyze protein levels of critical $\mathrm{Bcl}$ family members $(\mathrm{Bcl} 2,3, \mathrm{xl})$, and autophagy protein (Atg) family proteins, along with expression of TORC, during anoxia; through the complex itself (TORC; TOR, G $\beta$ L), and through activity of its inhibitor (PRAS40) and downstream target (p70S6K).

Hypothesis 3: Expression and activity of p53, that responds to DNA damage and triggers apoptosis, should be modulated under anoxia in a tissue specific manner to contribute to successful long term cell preservation in the hypometabolic state.

Hypothesis 4: Anoxia resistance and long term cell preservation in an anoxia-induced hypometabolic state will be promoted by inhibition of apoptosis in cells and, in an energy-limited state, by promotion of autophagy. 


\section{Cell cycle regulation (Chapter 6)}

The cell cycle is a critical regulatory target under stress conditions, due to both its high energetic needs and the control of proliferation in response to checkpoint signaling. In other models of oxygen limitation, there is a push to cell cycle attenuation culminating in cell quiescence at the $G_{1}$ stage.

Objectives:

1. Analyze the effects of anoxia exposure on cell cycle progression by looking at critical protein targets at the $\mathrm{G}_{1} / \mathrm{S}$ and $\mathrm{G}_{2} / \mathrm{M}$ barriers. Along with cyclin and CDK complex members, focus will be put on $G_{1}$ targets $R b$ and the E2F transcription factors. Protein levels and activity profiles of cell cycle inhibitors and control proteins will also be observed.

Hypothesis 5: In response to anoxia, cell cycle suppression will occur to reduce energy expenditures due to cellular proliferation. This may or may not involve a full transition into a state of cellular quiescence. 


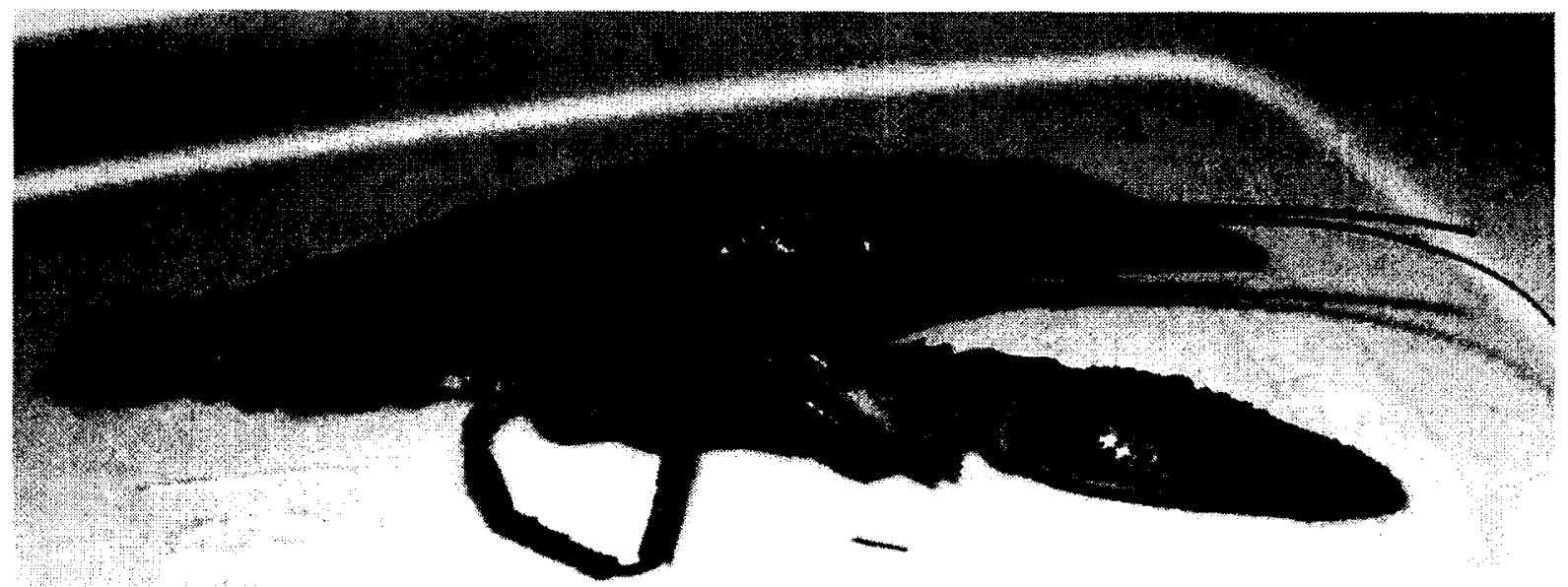

Figure 1.1: The freshwater crayfish, Orconectes virilis. Caught in the Rideau River (September 2005), Ottawa, Ontario. Photographed by J.M. Storey. 

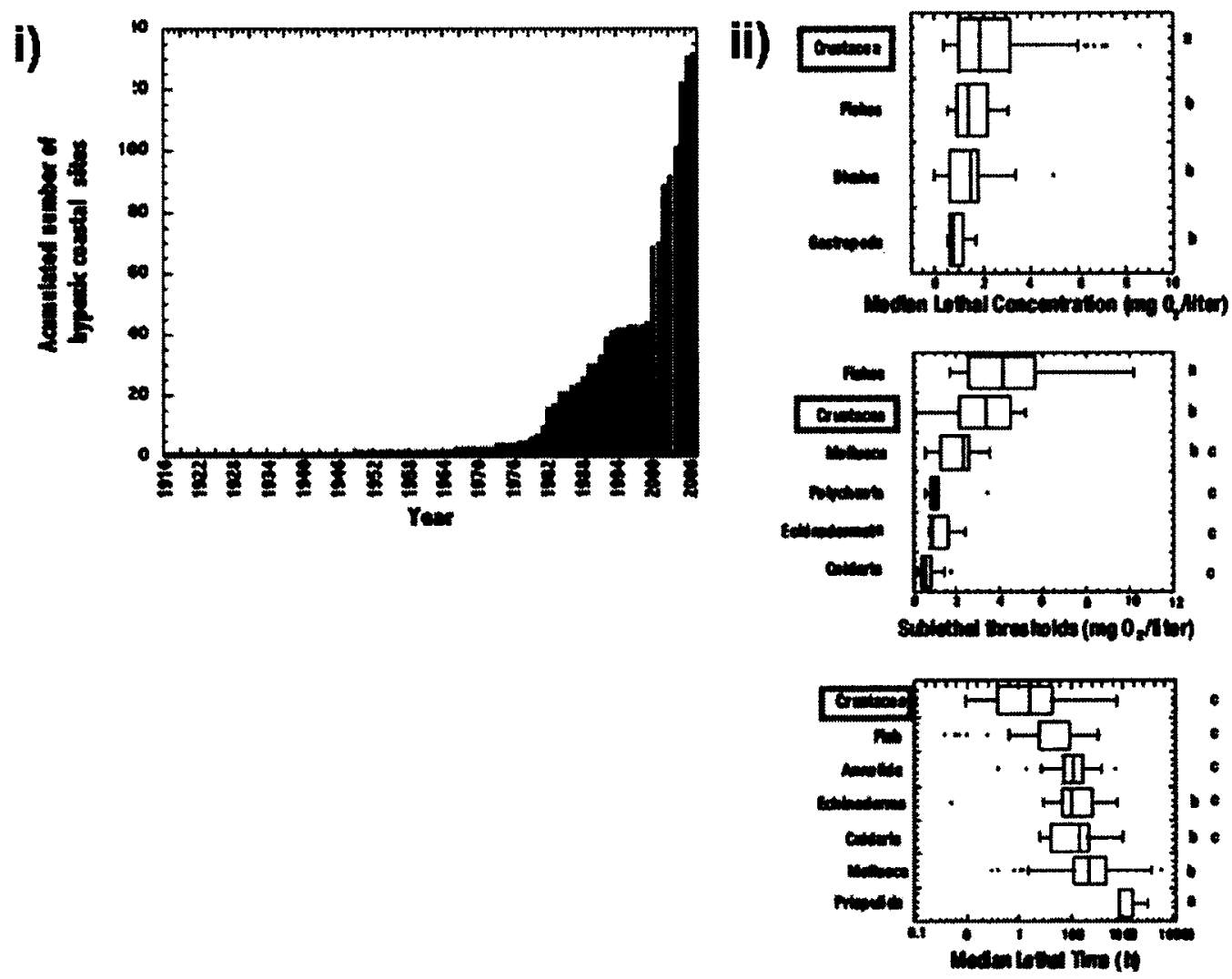

Figure 1.2: Hypoxic coastal regions and their effects on marine biodiversity. i) In just over 40 years there has been a drastic increase in hypoxic coastal zones, linked with global warming. ii) Tolerance to hypoxia in and oxygen thresholds of various marine taxa. Data from (Vaquer-Sunyer and Duarte, 2008). 


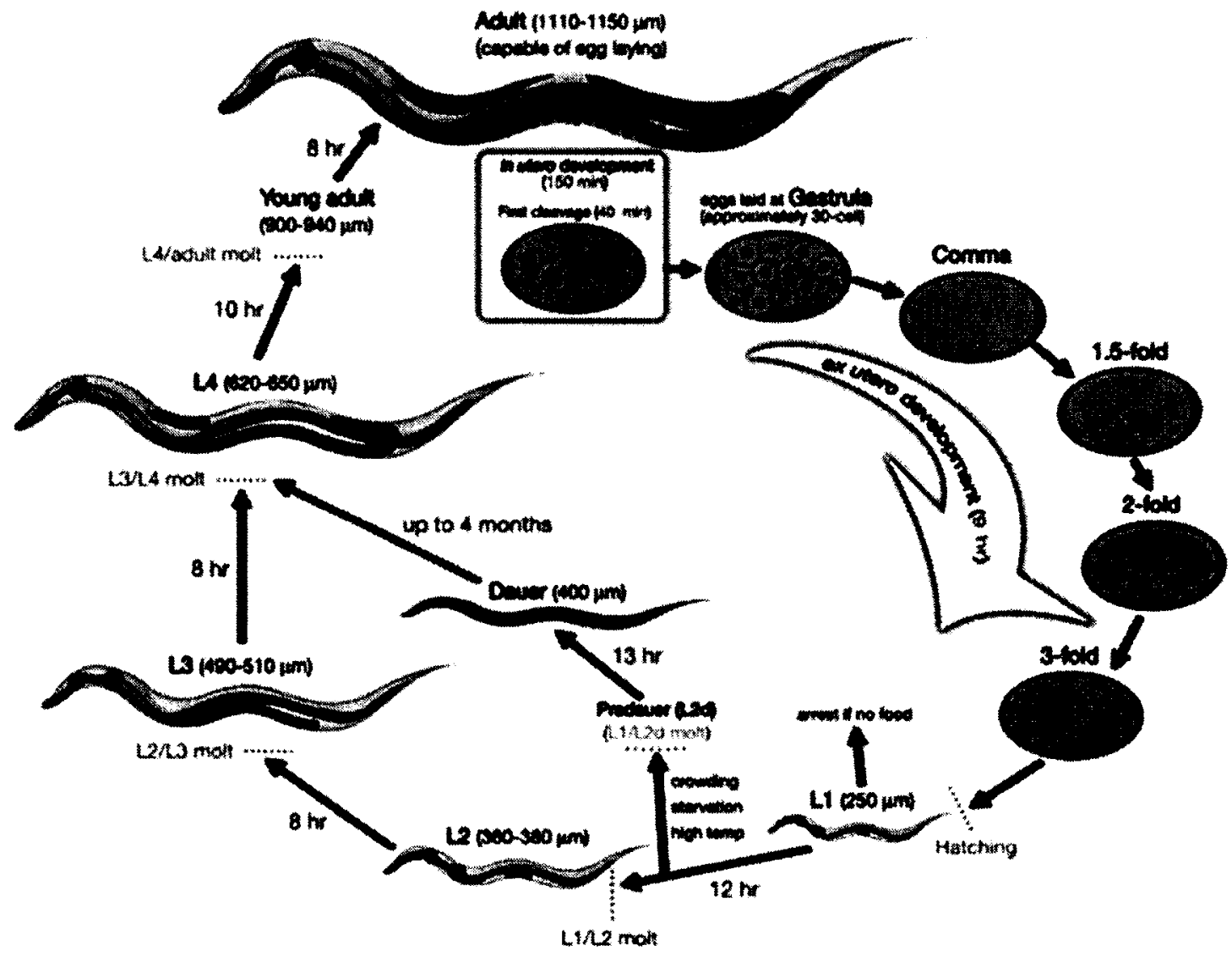

Figure 1.3: The life cycle of the nematode, $C$. elegans. This cycle indicates the relative timespan of the nematode development process as well as the positioning of entry into the dauer (hypometabolic) phase, which can extend the total lifespan of the worm significantly. Image from (Altun and Hall, 2005). 


\section{Chapter 2}

Regulation of Akt signaling, and its effect on the FoxO transcription factors during anoxia in the freshwater crayfish 


\subsection{Introduction}

As a stress adapted animal enters a period of hypometabolism, cellular responses are rapid and numerous. By altering major signaling pathways, the animal is able to reduce its metabolic rate, often up to $90 \%$ of its normal rate. Energy consuming processes like ion channel regulation and protein synthesis are minimized through networks of altered signal transduction. In order to regulate such a large variety of cellular processes, major protein kinases are often central to stress responsive signaling cascades.

\section{The Akt signaling response}

Amongst the major signaling protein kinases, protein kinase B (aka Akt), is one of the most multifaceted. As the hub of the insulin/insulin-like growth factor (IGF) response pathway, it is a largely pro-developmental/pro-survival signaling entity, and it mediates a range of critical cellular processes such as cell cycle control, apoptosis control, glycogen synthesis and antioxidant production (Osaki et al., 2004). Upstream of Akt, insulin/IGF ligands bind the insulin-like growth factor receptor (IGF-IR) that is a tyrosine kinase family receptor which autophosphorylates, and subsequently phosphorylates the phosphatidylinositol-3-kinase (PI3K). From here, phosphatidylinositol 4,5-bisphosphate (PIP2) is converted to phophatidylinositol-3,4,5-trisphosphate (PIP3), activating it. Active PIP3 phosphorylates the 3'-phosphoinositide dependent kinase-1 (PDK1), which in turn phosphorylates and activates Akt (FIG 2.1) (Osaki et al., 2004). Phosphorylation control of Akt is critical to its activity status, with phosphatases such as the "phosphatase and tensin homologue deleted on chromosome 10' (PTEN) and protein phosphatase 2A (PP2A) able to inactivate Akt and its downstream activity. PTEN specifically targets 
PIP3, so that downstream kinases (including PDK1 and Akt) cannot be phosphorylated (Jiang and Liu, 2008).

Active cytosolic Akt phosphorylates a number of target proteins including transcription factors and other kinases, activating or inhibiting them depending on their cellular role. One major target is glycogen synthase kinase 3 (GSK3). This serine/threonine kinase was initially discovered as a regulator of glycogen synthesis. Conversion of UDP-glucose with the addition of glucose monomers to create glycogen (requiring UTP in the process), is mediated by glycogen synthase, which is subject to inactivation by GSK3 (Ali et al., 2001). However, GSK3 is also now known to target a number of pro-apoptosis, pro-protein synthesis and pro-cell cycle progression targets for inhibition (Ali et al., 2001; Proud, 2007). Hence Akt activity, through GSK3 repression is very much pro-developmental.

Regulation of the FoxO transcription factors

Of the most important Akt targets, particularly implicated in stress responses, are the forkhead box (Fox) family of transcription factors. Within this highly conserved family of proteins, the subtype 'other' or 'FoxO' transcription factors are involved in transactivating numerous stress response genes, particularly in cases of oxidative stress (Storz, 2011). They are important regulators of cellular lifespan, with varied (and in terms of cellular survival, often contradictory) targets including pro-apoptosis genes, cell cycle inhibitor genes and antioxidant proteins. Within the FoxO subfamily, there are four functionally related proteins; FoxO1, FoxO3, FoxO4 and FoxO6, although this chapter will focus only on the key members, 1 and 3 . 
FoxO transcription factors are regulated by posttranslational modification, particularly phosphorylation. FoxOs can be phosphorylated on up to three sites, one of which is the nuclear localization signal (NLS), which prevents their entry into the nucleus (Burgering and Kops, 2002). Phosphorylation of FoxO is primarily mediated by Akt, but can also be carried out by the serum glucocorticoid kinase (SGK), which is a PI3K family member and is activated in parallel with insulin/IGF-Akt signaling (Brunet et al., 2001). SGK, along with being active in the cytoplasm, can translocate to the nucleus, where it is able to phosphorylate the FoxOs at the same sites as Akt; causing FoxOs to be anchored in the cytoplasm. Like Akt, cell survival signals trigger SGK activity, and when active, SGK1 (the family member studied in this chapter) preferentially targets FoxO3 (Liu et al., 2000).

Once phosphorylated, the FoxO proteins are often bound by 14-3-3 proteins, a family of regulatory chaperone proteins that are attracted to phosphorylated residues of particular targets. Binding of the 14-3-3 proteins, typically to the phosphorylated residue proximal to the NLS, causes the FoxO to be anchored in the cytoplasm. However, since both Akt (Andjelkovic et al., 1997) and SGK (Brunet et al., 2001) are able to translocate to the nucleus, and phosphorylate active FoxOs when inside, 14-3-3 has also been shown bind the phosphorylated transcription factor in the nucleus, resulting in its expulsion and localization in the cytoplasm.

While FoxOs are typically linked with cell cycle arrest and apoptotic signaling through their transactivation of target genes for both processes, they also regulate the expression of antioxidant genes (MnSOD and catalase). In cases of stress induction, cellular lifespan can be protected/extended as a result of stress activated FoxO activity, in 
part through up -regulation of antioxidant genes (Moskalev, 2008). Production of antioxidant proteins would be particularly pertinent in cases of recovery from limited oxygen, in which the electron transport chain can be overwhelmed by the influx of oxygen, which in turn significantly increases reactive oxygen species (ROS) production.

The regulation of FoxOs via Akt is not a novel discovery in stress-adapted models, and has been shown to be a critical bridge to the transition into the 'dauer' stage of the nematode Caenorhabditis elegans. During initiation of this period of developmental stasis, the nematode suppresses Akt signaling in order to promote FoxO (DAF-16) activity, particularly the capacity for cell cycle attenuation (Burgering and Kops, 2002). Although in the anoxia resistant crayfish, $O$. virilis, the period of stasis is not as metabolically stringent as in the nematode dauer stage, this point of regulation is nonetheless interesting to study.

In this chapter, the Akt signaling pathway, particularly with respect to downstream FoxO activity was analyzed in tissues of $O$. virilis. In response to anoxia, there was a strong pro-Akt signaling cascade seen from upstream, through PDK1 phosphorylation, and through to Akt and SGK phosphorylation. Downstream of Akt, GSK $3 \mathrm{a}$ was phosphorylated, along with evidence of continued phosphorylation of FoxO1 and 3, in some cases increased under anoxia. Despite this, increased DNA binding activity along with transcript and protein level increases of a FoxO target gene, coupled with decreased FoxO inhibitor 14-3-3 protein levels, indicate a continuing level of FoxO signaling amidst Akt activity.

\section{2 Materials and Methods}

2.2.1. Animals 
Freshwater crayfish (Orconectes virilis) were obtained from the Britannia Bait and Food market in Ottawa, Ontario. They were kept in aerated fresh water, in incubators set at $15^{\circ} \mathrm{C}$ for 7 days before use. Animals were then separated into groups of 10 and placed in individual covered buckets with $15^{\circ} \mathrm{C}$ water that was either aerated with a bubbler (control) or deoxygenated by bubbling with $100 \%$ nitrogen gas (bubbling with nitrogen gas was begun 45 minutes prior to adding animals - inducing acute anoxia; simulating natural crayfish migration into oxygen depleted water). For the purposes of this experiment, we have defined anoxia as the level of oxygen $\left(\leq 1 \% \mathrm{O}_{2} / 2\right.$ torr) resultant from 1 hour of $\mathrm{N}_{2}$ bubbling. Under these conditions (removal of $99 \% \mathrm{O}_{2}$ ), $\mathrm{O}_{2}$ concentration is measured as $0.0029 \mathrm{mM}$. Water is bubbled with $\mathrm{N}_{2}$ alone, and so $\mathrm{CO}_{2}$ levels are at $0.25 \%$ at most. Control animals were sampled from their buckets after 1 hour. Animals in deoxygenated water were sampled after 20 hours of anoxia exposure. Animals were killed by severing the head and then hepatopancreas was rapidly excised, immediately frozen in liquid nitrogen, and stored at $-70^{\circ} \mathrm{C}$ until use.

\subsubsection{RNA extraction}

All solutions and materials were treated with $0.1 \%$ diethylpyrocarbonate (DEPC) and autoclaved prior to use. Total RNA was isolated from tail muscle and hepatopancreas of normoxic (control) and $20 \mathrm{~h}$ anoxic crayfish using Trizol ${ }^{\mathrm{TM}}$ (Invitrogen). Briefly, $100 \mathrm{mg}$ of tissue was homogenized in $1 \mathrm{~mL}$ Trizol using a Polytron homogenizer. Subsequently, $200 \mu \mathrm{L}$ of chloroform was added and the homogenate was centrifuged at $10,000 \times g$ for 15 min at $4^{\circ} \mathrm{C}$. The upper aqueous phase (containing the RNA) was removed to a fresh tube and precipitated by the addition of $500 \mu \mathrm{L}$ isopropanol followed by incubation for $10 \mathrm{~min}$ at room temperature $(\mathrm{RT})$. The samples were centrifuged again at $10,000 \times g$ for 
$15 \mathrm{~min}$ at $4^{\circ} \mathrm{C}$. The total RNA pellet was washed with $1 \mathrm{~mL}$ of $70 \%$ ethanol and centrifuged again as above. The resulting pellet was air-dried for 10-15 min and resuspended in $50 \mu \mathrm{L}$ of DEPC-treated water. The quality of RNA was judged from the ratio of absorbances at 260 and $280 \mathrm{~nm}$ as well as gel electrophoresis on a $1 \%$ agarose gel stained with ethidium bromide to check for the integrity of $18 \mathrm{~S}$ and $28 \mathrm{~S}$ ribosomal RNA bands.

\subsubsection{Primers}

Primers were synthesized by Sigma Genosys. Forward and reverse primers for all genes were designed based on conserved regions of these genes as determined from a DNA alignment of sequences from other species including human (Homo sapiens), mouse (Mus musculus), western clawed frog (Xenopus tropicalis), round worm (C. elegans) and water flea (Daphnia pulex). For tubulin, myosin heavy chain (mhc) and MnSOD, perfect (species-specific) primers were designed based on the retrieved crayfish-specific sequence after initial PCR trials and these were used for quantification. The primers and their conditions are listed in Table 2.1.

\subsection{4. cDNA synthesis and PCR}

A $4 \mu \mathrm{g}$ aliquot of RNA was diluted with DEPC water to $10 \mu \mathrm{L}$ final volume and used to synthesize cDNA. Briefly, $1 \mu \mathrm{L}$ of $200 \mathrm{ng} / \mu \mathrm{L}$ Oligo $d T\left(5^{\prime}-\right.$

TTTTTTTTTTTTTTTTTTTTTTV-3') primer was added to the RNA sample and incubated at $65^{\circ} \mathrm{C}$ for $5 \mathrm{~min}$. The mixture was then chilled rapidly on ice and $4 \mu \mathrm{L} 5 \mathrm{X}$ first strand buffer, $2 \mu \mathrm{L} 100 \mathrm{mM}$ DTT, $1 \mu \mathrm{L}$ dNTP mixture (10 mM each) and $1 \mu \mathrm{L}$ reverse transcriptase enzyme M-MLV (all reagents from Invitrogen) were added for a total volume of $19 \mu \mathrm{L}$. The mix was incubated at $42^{\circ} \mathrm{C}$ for $1 \mathrm{~h}$ and chilled to $4^{\circ} \mathrm{C}$. The 
resulting cDNA was diluted $\left(10^{-1}\right.$ to $\left.10^{-3}\right)$ and amplified by polymerase chain reaction (PCR) with the forward and reverse primers mentioned above. The PCR reaction of $25 \mu \mathrm{L}$ final volume was composed by mixing $15 \mu \mathrm{L}$ of sterile water, $5 \mu \mathrm{L}$ of diluted cDNA, $1.25 \mu \mathrm{L}$ of primer mixture (to a final concentration of $1.5 \mu \mathrm{M}$ forward and $1.5 \mu \mathrm{M}$ reverse), $0.5 \mu \mathrm{L}$ of $10 \times \mathrm{PCR}$ buffer (Invitrogen), $1.75 \mu \mathrm{L}$ of $50 \mathrm{mM} \mathrm{MgCl}, 0.5 \mu \mathrm{L}$ of $\mathrm{dNTP}$ mixture (10 $\mathrm{mM}$ each) and $1 \mu \mathrm{L}$ of Taq Polymerase. The PCR program was as follows: $7 \mathrm{~min}$ at $94^{\circ} \mathrm{C}$ followed by 38 cycles of $1 \mathrm{~min}$ at $94^{\circ} \mathrm{C}, 1 \mathrm{~min}$ at a predetermined annealing temperature $\left(52^{\circ} \mathrm{C}\right.$ for $\mathrm{MnSOD}, 53^{\circ} \mathrm{C}$ for tubulin, $58^{\circ} \mathrm{C}$ for $\mathrm{mhc}$ ), and $1.5 \mathrm{~min}$ at $72^{\circ} \mathrm{C}$. The final step was $72^{\circ} \mathrm{C}$ for $10 \mathrm{~min}$. PCR products were separated on a $1 \%$ agarose gel stained with ethidium bromide, visualized using the ChemiGenius imaging system (Syngene, Frederick, MD, USA) under UV light and quantified using the GeneTools program. The bands from the most dilute cDNA sample that gave visible product were used for quantification purposes to make sure that the products had not reached amplification saturation.

\subsubsection{Sequencing}

PCR products were sequenced by DNA Landmarks (St.-Jean-sur-Richelieu, QC).

Sequences were verified as encoding the correct genes using the program BLASTN at the NIH (http://www.ncbi.nlm.nih.gov/blast). The sequences were translated using DNAMAN. Each protein sequence was aligned with the respective sequences from other animals, and homology trees were generated based on that alignment using DNAMAN.

\subsubsection{Protein homogenates and western blotting}

Frozen tissue samples were crushed under liquid nitrogen and then homogenized 1:5 w:v in homogenizing buffer $\mathrm{C}(20 \mathrm{mM}$ Hepes, $\mathrm{pH} 7.5,200 \mathrm{mM} \mathrm{NaCl}, 0.1 \mathrm{mM}$ EDTA, 
$10 \mathrm{mM} \mathrm{NaF}, 1 \mathrm{mM} \mathrm{Na}_{3} \mathrm{VO}_{4}, 10 \mathrm{mM} \beta$-glycerophosphate) with a few crystals of PMSF and $1 \mu \mathrm{L}$ protease inhibitor cocktail added (Sigma-Aldrich, Oakville, ON, CA). Samples were centrifuged as above for $15 \mathrm{~min}$ and supernatant was collected. Soluble protein concentration was measured using the Coomassie Blue dye-binding method with a prepared reagent (Bio-Rad, Hercules, CA, USA) and bovine serum albumin as the standard. Sample concentrations were adjusted to a constant $10 \mu \mathrm{g} / \mu \mathrm{L}$ by the further addition of small amounts of homogenizing buffer and then aliquots of samples were mixed 1:1 v:v with $2 \times$ SDS loading buffer ( $100 \mathrm{mM}$ Tris-base, $4 \% \mathrm{w} / \mathrm{v}$ SDS, $20 \% \mathrm{v} / \mathrm{v}$ glycerol, $0.2 \% \mathrm{w} / \mathrm{v}$ bromophenol blue, $10 \% \mathrm{v} / \mathrm{v} 2$-mercaptoethanol) to give a final protein concentration of $5 \mu \mathrm{g} / \mu \mathrm{L}$. Samples were boiled for $5 \mathrm{~min}$ and chilled on ice prior to loading onto gels.

Aliquots containing $25 \mu \mathrm{g}$ protein were loaded into wells of $10 \%$ polyacrylamide gels, together with prestained molecular weight standards (Bio-Rad) and separated using a discontinuous buffer system (Laemmli, 1970). Samples from all experimental conditions were always run on the same gel. Gels were run (in running buffer; 10 fold diluted [75.8g Tris-Base, $470 \mathrm{~g}$ Glycine, $25 \mathrm{~g}$ SDS, adjusted to $\mathrm{pH} 8-8.5$ and diluted to $2 \mathrm{~L}$ with $\left.\mathrm{ddH}_{2} \mathrm{O}\right]$ ) in a Mini-Protean III apparatus (Bio-Rad) at constant $180 \mathrm{~V}$ for 45 minutes at RT. Proteins were transferred onto PVDF membranes at $60 \mathrm{~V}$ for 90 minutes at $4^{\circ} \mathrm{C}$. The resulting blots were probed overnight with primary antibodies (Table 2.2) diluted 1:1000 v:v in Tris Buffered Saline and Tween 20 (TBST). TBST was comprised of a 1L 10 fold dilution of TBS buffer [24.2 Tris Base ( $\mathrm{pH} 7.6), 80 \mathrm{~g} \mathrm{NaCl}$, and topped up to $1 \mathrm{~L}$ volume with $\mathrm{ddH}_{2} \mathrm{O}$ ] with an addition of $0.5 \mathrm{ml}$ Tween-20. Blots were then washed multiple times with TBST and incubated with HRP-linked goat anti-rabbit 
IgG (diluted 1:4000 in TBST) (BioShop, Burlington, ON, CA) for 60 minutes at RT.

Blots were then washed with TBST and developed using enhanced chemiluminescence reagents. Subsequently, blots were stained with Coomassie blue stain $(0.25 \% \mathrm{w} / \mathrm{v}$ brilliant blue $\mathrm{R}, 7.5 \% \mathrm{v} / \mathrm{v}$ acetic acid, $50 \% \mathrm{v} / \mathrm{v}$ methanol and $42.5 \% \mathrm{ddH}_{2} \mathrm{O}$ ) for 10 minutes and destained with destain solution (10\% v:v acetic acid, $30 \%$ v:v methanol and $60 \%$ v:v ddH2O), for a further 10 minutes.

\subsubsection{Preparation of nuclear extracts}

Nuclear extracts were prepared using a slight modification of the method described by Dignam et al. (1983). Briefly, $0.5 \mathrm{~g}$ samples of frozen tissue were homogenized using a Dounce homogenizer in $1 \mathrm{~mL}$ of homogenization buffer $(10 \mathrm{mM}$ Hepes, $10 \mathrm{mM} \mathrm{KCl}$, $10 \mathrm{mM}$ EDTA, $1 \mathrm{mM}$ DTT, pH 7.9). The DTT, as well as $10 \mu \mathrm{L}$ of Protease Inhibitor Cocktail (Sigma) were added just prior to homogenization. Samples were centrifuged at $10,000 \times g$ for $10 \mathrm{~min}$ at $4^{\circ} \mathrm{C}$ and the supernatant (cytoplasmic extract) was removed. The pellet was resuspended in $150 \mu \mathrm{L}$ of extraction buffer (20 mM HEPES, pH 7.9; $400 \mathrm{mM}$ $\mathrm{NaCl} ; 1 \mathrm{mM}$ EDTA; $10 \% \mathrm{v} / \mathrm{v}$ glycerol; $1 \mathrm{mM}$ DTT). The DTT, as well as $1.5 \mu \mathrm{L}$ of Protease Inhibitor Cocktail (Sigma) were added just prior to addition of the buffer to the pellet. Tubes containing the samples were put on ice horizontally on a rocking platform for $1 \mathrm{~h}$. Samples were then centrifuged at $10,000 \times \mathrm{g}$ for $10 \mathrm{~min}$ at $4^{\circ} \mathrm{C}$. Supernatants (nuclear extract) were collected and treated as described above to create samples for western immunoblotting, with the exception of the final concentration being adjusted to 2 $\mu \mathrm{g} / \mu \mathrm{l}$. The integrity of the nuclei was confirmed by immunoblotting of cytoplasmic and nuclear fractions and probing with a histone $\mathrm{H} 3$ antibody specific for animals ranging from Drosophila to mammals (Cell Signaling; diluted 1:1000 in TBST). 


\subsubsection{DNA-binding assay}

Nuclear extracts were prepared as above (final concentration $2 \mu \mathrm{g} / \mu \mathrm{l}$ ), with extraction buffer without further manipulation. Aliquots containing equal amounts of protein from each sample (10-20 $\mu \mathrm{g} / \mathrm{well}$, depending on tissue) were then used to assess the amount of binding by FoxO1 and FoxO3 to the FOXO response element. This was done using an ELISA-type assay that uses 96-well microplates that were previously coated with an oligonucleotide corresponding to the DNA binding element that is recognized by FoxOl/3.

The sequence of the biotin-conjugated probe was 5'- BiotinGACTCGAGTTGTTTACATAGGATGCG-3', whereas the complementary sequence was 5'- CGCATCCTATGTAAACAACTCGAGTC- 3'. Both oligonucleotides were purchased from Sigma Genosys, diluted to $500 \mu \mathrm{M}$ using sterile $\mathrm{H}_{2} \mathrm{O}$ and subsequently mixed 1:1 (v/v) for a total volume of $20 \mu \mathrm{l}$. Probes were then placed in a thermocycler for $10 \mathrm{~min}$ at $95^{\circ} \mathrm{C}$ and the temperature of the block was slowly cooled to RT. A $40 \mathrm{pmol}$ aliquot of double-stranded, biotinylated probe in $50 \mu \mathrm{L}$ of phosphate buffered saline (PBS; $137 \mathrm{mM} \mathrm{NaCl}, 2.7 \mathrm{mM} \mathrm{KCl}, 10 \mathrm{mM} \mathrm{Na}_{2} \mathrm{HPO}_{4}, 2 \mathrm{mM} \mathrm{KH}_{2} \mathrm{PO}_{4}, \mathrm{pH}$ 7.4) was then added to each well of a streptavidin-coated microplate (VWR). The plate was then incubated at RT for $1 \mathrm{~h}$ and washed twice with wash buffer (0.1\% Tween-20 in PBS) and once with PBS. Aliquots of 10-16 $\mu \mathrm{g}$ protein were combined with $50 \mu \mathrm{L}$ of $1 \times$ protein binding buffer [ $10 \mathrm{mM}$ Hepes, pH 7.9, $50 \mathrm{mM} \mathrm{KCl}, 0.5 \mathrm{mM}$ EDTA, $3 \mathrm{mM} \mathrm{MgCl}_{2}, 10 \%$ glycerol, $0.5 \mathrm{mg} / \mathrm{mL}$ BSA, $0.05 \%$ NP-40, 1-2 $\mu$ g salmon sperm DNA (BioShop), $0.5 \mathrm{mM}$ DTT, and $\mathrm{NaCl}$ (a variable amount was used based on the volume of nuclear extract added, final concentration was always $40 \mathrm{mM}$ )] and then added to the wells. Equal 
amounts of protein for all experimental conditions were used in each assay, and all experimental samples were always run together. Only binding buffer, without protein samples, was added to the negative control wells. The plate was incubated at RT with mild agitation for $75 \mathrm{~min}$ followed by 4 washes with wash buffer. A $60 \mu \mathrm{L}$ aliquot of the FoxO1 or FoxO3 antibody (the same one used for western blotting), diluted 1:1000 in PBS was added to the wells and the plate was incubated for $1 \mathrm{~h}$. The plate was washed as above and then $60 \mu \mathrm{L}$ of anti-rabbit IgG-HRP (BioShop) diluted 1:2000 in PBS was added and the plate was incubated for $1 \mathrm{~h}$. Following this, the plate was washed as above and $60 \mu \mathrm{L}$ of TMB (tetramethylbenzidine) was added (Bioshop). Once colour had developed, the reaction was stopped by the addition of $1 \mathrm{M} \mathrm{HCl}$ and optical density was read at $450 \mathrm{~nm}$ with a reference wavelength of $655 \mathrm{~nm}$ using a Multiskan Spectrum (Thermo Labsystems).

\subsubsection{Electromobility Shift Assay}

In order to confirm probe efficiency for the DNA-binding activity ELISA, an electromobility shift assay was performed to visualize probe binding. First, biotinylated probe from the DNA binding activity assay $(250 \mathrm{pmol} / \mu \mathrm{l})$ was diluted to $10 \mathrm{pmol} / \mu \mathrm{l}$. Aliquots of $4 \mu \mathrm{l}(8 \mu \mathrm{g})$ of nuclear extract were mixed with $1 \mu \mathrm{LEPC}$ treated $\mathrm{H}_{2} \mathrm{O}$ and 4 $\mu$ l EMSA master mix (5X EMSA binding buffer [50 mM Tris $\mathrm{HCl}, \mathrm{pH} 7.8,250 \mathrm{mM}$ $\mathrm{NaCl}, 5 \mathrm{mM}$ EDTA and $25 \%$ glycerol] combined with $1 \mu \mathrm{g} / \mu \mathrm{l} \mathrm{Poly} \mathrm{d(I-C)} \mathrm{in} \mathrm{a} 1: 1 \mathrm{v}: \mathrm{v}$ ratio) for a total volume of $9 \mu \mathrm{l}$. Samples were vortexed and incubated at RT for 5 minutes. A $1 \mu \mathrm{l}$ aliquot of TF probe (FoxO specific) was added and the mixture was vortexed again before placement in the thermocycler for 30 minutes at $15^{\circ} \mathrm{C}$. The positive control was biotinylated E2F Probe (Panomics EMSA Kit 7104) tested with turtle 
(Trachemys scripta elegans) liver, in the same mix as above. Negative controls for no protein and no probe were also created, with water volume making up the total volume.

Samples were mixed with $1 \mu 1$ 6x DNA loading dye (BioShop) prior to loading on a $6 \%$ non-denaturing polyacrylamide gel [ $1 \mathrm{ml} 10 \mathrm{X}$ TBE (chilled), $4 \mathrm{ml} 30 \%$ bisacrylamide, $625 \mu \mathrm{l} 80 \%$ glycerol, $14.375 \mathrm{ml}$ deionized sterile $\mathrm{H}_{2} \mathrm{O}, 300 \mu 1$ 10\% APS and $20 \mu 1$ TEMED giving a total of $2 \times 10 \mathrm{ml} / \mathrm{gel}$ ). The gels were cast as in the Western blotting procedure (above). Gels were pre-un in chilled 0.5x TBE (diluted from 10X TBE [108g Tris Base, 55g Boric acid, 40ml 0.5M EDTA ( $\mathrm{pH} \mathrm{8.0)}$ topped up to $1 \mathrm{~L}$ volume with $\left.\mathrm{ddH}_{2} \mathrm{O}\right]$ ) for 10 minutes at $120 \mathrm{~V}$. Samples were loaded into the gels and run at $120 \mathrm{~V}$ for 70 minutes at $4^{\circ} \mathrm{C}$ (fridge).

Prior to transfer, membranes (Pall Biodyne B nylon membrane) were pre-soaked in 0.5x TBE. Gels were sandwiched to membranes, and then transferred in $0.5 \times$ TBE (chilled) for 30 minutes at $300 \mathrm{~mA}$, at $4^{\circ} \mathrm{C}$. Once transfer was completed, the membrane was stored between dry filter paper, and the oligos were allowed to fix to the membrane in the oven $\left(80^{\circ} \mathrm{C}\right)$ for 60 minutes. Following this, the membranes were washed on a rocking platform with $5 \mathrm{ml}$ (per membrane) of 1x EMSA Blocking Buffer (diluted from Panomics CS7535) for 30 minutes at RT. The blocking buffer was decanted and $5 \mu 1 / \mathrm{ml}$ Streptavidin-HRP mixture (BioShop) was added to it. The mixture was then reapplied to the membrane and left to incubate on a rocking platform for a further 30 minutes at RT. Finally, the membranes were washed 3 times, consecutively, with $1 \mathrm{x}$ wash buffer (diluted from Panomics 7537). The membranes were then exposed using ECL solutions as in the Western blotting protocol.

\subsubsection{Data and statistics}


Bands (from agarose or SDS PAGE gels) were scanned using a ChemiGenius BioImaging system and densitometric analysis was performed using the associated GeneTools software (Syngene, Frederick, MD). RT-PCR bands for all genes were normalized against bands for $\alpha$-tubulin amplified from the same cDNA sample. To control for irregularities in SDS PAGE gel loading, the chemiluminescent band intensity in each lane was normalized against the corresponding density of one Coomassie stained protein band, in the same lane, which was seen to be constant between all control and experimental conditions (these bands were well separated from the target band).

Examples of constantly expressed proteins are listed in Appendix F. To normalize ELISA results, the $\mathrm{OD}$ from the negative control was subtracted from the $\mathrm{OD}$ of each sample. Mean normalized band densities \pm SEM were then calculated; statistical analysis used the Student's t-test. 
Table 2.1 List of Primers used in Chapter 2

\begin{tabular}{|c|c|c|c|}
\hline Gene/primer & Primer sequence (5'-3') & $\begin{array}{l}\text { Annealing } \\
\text { temp. } \\
\left({ }^{\circ} \mathrm{C}\right)\end{array}$ & $\begin{array}{c}\text { Expected } \\
\text { product } \\
\text { size } \\
\text { (bp) } \\
\end{array}$ \\
\hline Tubulin perfect forward & GACATTTGTTCATGGTATGC & \multirow[b]{2}{*}{53} & \multirow[b]{2}{*}{$\sim 350$} \\
\hline Tubulin perfect reverse & TGTCGGTGGAATAGGGAAAG & & \\
\hline$M H C$ perfect forward & TCTTCGTCGACTTCGGTATG & \multirow[t]{2}{*}{58} & \multirow[t]{2}{*}{$\sim 600$} \\
\hline$M H C$ perfect reverse & CAGGTCAGCTGGTGCATGAT & & \\
\hline$M n S O D$ perfect forward & GGAGATCCAYCACACAAARC & \multirow[b]{2}{*}{52} & \multirow[b]{2}{*}{$\sim 200$} \\
\hline$M n S O D$ perfect reverse & GTCCARAAGATGGTRTGGTT & & \\
\hline
\end{tabular}

Table 2.2 List of primary antibodies used in Chapter 2

\begin{tabular}{|l|l|l|}
\hline \multicolumn{1}{|c|}{$\begin{array}{c}\text { Primary } \\
\text { Antibody }\end{array}$} & \multicolumn{1}{c|}{ Company } & Catalogue \# \\
\hline Akt & Cell Signaling (Danvers, MA, USA) & $\# 9272$ \\
\hline Akt- $p$ (T-308) & Cell Signaling (Danvers, MA, USA) & $\# 2965$ \\
\hline PDK1-p(S- 241) & Cell Signaling (Danvers, MA, USA) & $\# 3061$ \\
\hline Sgk & Cell Signaling (Danvers, MA, USA) & $\# 3272$ \\
\hline Sgk-p(S-78) & Cell Signaling (Danvers, MA, USA) & $\# 3271$ \\
\hline FoxOI & Cell Signaling (Danvers, MA, USA) & $\# 2880$ \\
\hline FoxO1-p (S-256) & Cell Signaling (Danvers, MA, USA) & $\# 9461$ \\
\hline FoxO3 & Cell Signaling (Danvers, MA, USA) & $\# 2497$ \\
\hline FoxO3-p (S-253) & Cell Signaling (Danvers, MA, USA) & $\# 9466$ \\
\hline Gsk3a & Cell Signaling (Danvers, MA, USA) & $\# 4337$ \\
\hline Gsk3a-p (S-21) & Cell Signaling (Danvers, MA, USA) & $\# 9316$ \\
\hline $14-3-3$ & Santa Cruz Biotechnology (Santa Cruz, CA, & sc-166473 \\
& USA) & \\
\hline MnSOD & Genscript (Piscataway, NJ, USA) & $\mathrm{A} 01005$ \\
\hline Catalase & Genscript (Piscataway, NJ, USA) & A01187 \\
\hline PTEN & Cell Signaling (Danvers, MA, USA) & $\# 9559$ \\
\hline
\end{tabular}




\subsection{Results}

\subsubsection{Technique optimization}

Optimization of PCR includes identifying the appropriate annealing temperature for each transcript type $\left(52^{\circ} \mathrm{C}\right.$ for $M n S O D, 53^{\circ} \mathrm{C}$ for tubulin, $58^{\circ} \mathrm{C}$ for $\left.m h c\right)$ and determining the optimal number of cycles to use to ensure linearity. The effect of cycle number is shown in FIG 2.2 which shows the normal number of cycle used (38) in comparison with a higher number of cycles (40) that gave significantly brighter (more saturated) bands, and a lower number of cycles $(34,36)$ that resulted in the band disappearance. Dilutions of cDNA samples were also amplified in order to find the optimal dilution that gave visible product for quantification of each gene without reaching amplification saturation. FIG

2.3 shows a typical result for a dilution series for tubulin amplification ranging from $10^{-2}$ to $10^{-6}$ dilution and amplified over 40 cycles. In this case, a $10^{-3}$ dilution proved to be the lowest usable concentration. RT-PCR band densities for all genes studied in this thesis were normalized against bands for $\alpha$-tubulin amplified from the same cDNA sample. FIG 2.4 provides information on the tubulin-like PCR product amplified from crayfish and shows that amplicon levels remained constant between normoxic (control) and anoxic conditions in both tail muscle and hepatopancreas. As a further confirmation that the tubulin-like product was a valid standard for normalization, tubulin levels were also checked against myosin heavy chain that was also amplified from the same samples (FIG 2.5). Transcript levels of $m h c$ also remained constant between aerobic and anoxic conditions. It is interesting to note that the crayfish $m h c$ nucleotide and deduced amino acid sequences showed very high homology with other arthropods. 


\subsubsection{Protein expression, phosphorylation state, and cellular localization of Akt in} response to anoxia

Changes in the total protein levels of Akt and the amount of phosphorylated Akt (Threonine 308) in response to anoxia were assessed using immunoblotting in two crayfish tissues: tail muscle and hepatopancreas (FIG.2.6). Akt is activated by phosphorylation via upstream canonical signaling from the insulin/IGF pathway. The polyclonal Akt and Akt (T308) antibodies both cross-reacted with protein bands of $\sim 60$ $\mathrm{kDa}$ which is the expected size of Akt. Akt protein levels increased in both tissues in response to $24 \mathrm{~h}$ of anoxia $\left(\mathrm{N}_{2}\right.$ bubbled water); in tail muscle levels rose by $1.5 \pm 0.2$ fold and in hepatopancreas the increase was $2.8 \pm 0.4$ fold (both $P<0.05$ ). Phosphorylation of Akt (T308) also increased significantly under anoxia. Tail muscle Akt exhibited a $1.7 \pm$ 0.2 fold increase in phosphorylation, whereas phospho-Akt (T308) increased in hepatopancreas by $1.9 \pm 0.07$ fold (both $P<0.05$ ) over control values. The amount of phosphorylated Akt was also measured in nuclear extracts of both crayfish tissues (FIG. 2.7). However, nuclear phospho-Akt (T308) did not change under anoxia.

\subsubsection{Protein expression of PDK1 (S241) in response to anoxia}

PDK1, one of the upstream kinases that can activate Akt by phosphorylation, must be phosphorylated itself as part of canonical insulin/IGF pathway signaling. Changes in phosphorylation of serine 241 in response to anoxia were assessed using immunoblotting (FIG 2.8). Polyclonal PDK 1 (S241) antibodies cross reacted with bands of $\sim 65 \mathrm{kDa}$, the expected size. Phosphorylation levels were unchanged in the hepatopancreas but increased significantly by $1.4 \pm 0.04$ fold $(\mathrm{P}<0.05)$ in tail muscle under anoxic conditions. 


\subsubsection{Protein expression of PTEN phosphatase in cytoplasmic and nuclear fractions}

PTEN phosphatase, a negative regulator of Akt via dephosphorylation, has roles dependent on its cellular location (Song et al., 2011). Changes in PTEN protein expression in cytoplasmic and nuclear fractions were assessed using immunoblotting (FIG 2.9). Polyclonal PTEN antibodies cross reacted with bands of $\sim 55 \mathrm{kDa}$, which is their expected size. PTEN protein levels did not vary in the cytoplasmic fractions, but under anoxia, there were significant increases in nuclear PTEN content. In the tail muscle protein levels increased $3.0 \pm 0.2$ fold, while in the hepatopancreas protein levels increased $2.8 \pm 0.2$ fold (both $P<0.05$ ).

\subsubsection{Protein expression and phosphorylation of GSK3a in response to anoxia}

As a target of Akt suppression, GSK3a can be phosphorylated on its serine 21 residue (Ali et al., 2001). Changes in both protein levels and phosphorylation levels were assessed in both tissues, in response to anoxia (FIG 2.10). Polyclonal GSK3a and phospho-GSK3a (S21) antibodies cross reacted with bands of $\sim 50 \mathrm{kDa}$, which is their expected size. While protein levels of GSK3a increased somewhat in tail muscle (1.2 \pm 0.01 fold; $P<0.05$ ) under anoxia, they remained static in the hepatopancreas. However, phosphorylation levels increased in both tissues, correlated with the active Akt profile. In the tail muscle, phosphorylation of GSK3a (S21) increased $1.3 \pm 0.06$ fold in anoxia, while in the hepatopancreas, levels rose $1.7 \pm 0.05$ fold (both $\mathrm{P}<0.05$ ).

\subsubsection{Protein expression and phosphorylation of SGK1 in response to anoxia}

A PI3K family kinase related to Akt, SGK1 shares some of Akt's downstream targets and also requires phosphorylation to become active. Changes in both protein levels and phosphorylation levels were assessed in both tissues, in response to anoxia, using 
immunoblotting (FIG 2.11). Polyclonal SGK1 and phospho-SGK1 (S78) antibodies cross reacted with bands of $\sim 55 \mathrm{kDa}$, the expected size. SGK1 protein levels increased significantly by $1.7 \pm 0.3$ fold in tail muscle and $1.6 \pm 0.2$ fold in hepatopancreas (both $P$ $<0.05$ ) in response to anoxia. However, the content of phospho-SGK1 (S78) only rose significantly in tail muscle by $1.6 \pm 0.1$ fold under anoxia.

\subsubsection{Protein expression and cellular distribution of FoxO 1 and FoxO3 in response to} anoxia

Changes in protein levels of FoxOl and FoxO3 were assessed in both tissues, in response to anoxia (FIG 2.12). Polyclonal FoxOl and FoxO3 antibodies cross reacted with bands of $\sim 80$ and $90 \mathrm{kDa}$, respectively, which are their expected sizes. Protein levels of FoxO1 remained unchanged, under anoxia, in the hepatopancreas, while they increased in the tail muscle $(1.4 \pm 0.04$ fold; $\mathrm{P}<0.05)$. FoxO3 Protein levels, however, did not change at all in the transition between control and anoxic conditions.

FoxO transcription factors need to be present in the nucleus in order to transactivate their target genes. Phosphorylation and binding to 14-3-3, however, keeps them anchored in the cytoplasm. Changes in protein levels of FoxO1 (FIG 2.13) and FoxO3 (FIG 2.14) in cytoplasmic and nuclear fractions were assessed in response to anoxia. FoxOl protein levels in the cytoplasmic fraction of both tail muscle and hepatopancreas increased significantly (by $1.8 \pm 0.2$ fold and $2.5 \pm 0.1$ fold, respectively; both $P<0.05$ ), whereas no increases were seen in the nuclear fractions. In fact, while levels remained static in the hepatopancreas, in the tail muscle nuclear fraction, FoxO1 protein levels dropped by $60 \pm 8 \%(\mathrm{P}<0.05)$ under anoxia. 
FoxO3 levels in the tail muscle decreased by $25 \pm 5 \%$ in cytoplasmic fractions, while in the nucleus, protein levels increased by $1.4 \pm 0.08$ fold under anoxia (both $\mathrm{P}<$ 0.05). In the hepatopancreas, however, there were no significant changes in FoxO3 protein levels in either cytoplasmic or nuclear fractions under anoxia.

\subsubsection{Phosphorylation of FoxOl and 3 in response to anoxia}

Along with profiling subcellular distribution, analysis of the phosphorylation state of FoxOs is a major indicator of their transcription factor activity. Capable of being phosphorylated by Akt and SGK1, phosphorylated FoxO is unable to enter the nucleus, or if phosphorylated in the nucleus, is targeted for expulsion (in conjunction with 14-3-3 binding); both responses significantly reduce FoxO transactivation capacity.

Phosphorylation of serine 256 on FoxO1 (FIG 2.15) and serine 253 (FIG 2.16) on FoxO3 was assessed in both tissues, in cytoplasmic and nuclear fractions in response to anoxia. Polyclonal FoxO1-p (S256) and FoxO3-p (S253) antibodies cross reacted with bands of $\sim 80$ and $90 \mathrm{kDa}$ (as above).

In tail muscle, phosphorylation of FoxO1 increased in the cytoplasmic fraction by $2.1 \pm 0.2$ fold $)(\mathrm{P}<0.05)$, whereas the amount of phosphorylated FoxO1 decreased in the nuclear fraction by $30 \pm 10 \%(P<0.05)$ under anoxia. In the hepatopancreas, there were no changes in phosphorylation under anoxia in the cytoplasmic fractions, but a significant increase occurred in the nuclear fraction $(1.8 \pm 0.1$ fold; $\mathrm{P}<0.05)$.

Phosphorylation of FoxO3 at serine 253 was unaffected in the cytoplasmic fraction of tail muscle, but a significant increase was seen in the nuclear fraction $(1.8 \pm$ 0.1 fold; $P<0.05)$ under anoxia. In the hepatopancreas, again, there were no changes in 
cytoplasmic FoxO3 phosphorylation, but a decrease in the nuclear fraction occurred by $35 \pm 4 \%(P<0.05)$.

\subsubsection{DNA Binding activity of FoxOl and 3 in response to anoxia}

Changes in the DNA-binding activity of nuclear FoxO1 and FoxO3 in response to anoxia were assessed using a transcription factor binding assay (FIG.2.17). The ELISA assay detects relative binding of the target protein (within a nuclear extract) to a probe designed to emulate the recognition sequence of the transcription factor that would be present in promoter sites of specific genes. The DNA binding assay is quantified through the subsequent interactions of antibodies with the transcription factor, and the production of a colourimetric response. The EMSA is designed to visualize the transcription factor-probe interaction, in order to confirm probe effectiveness. Despite decreased protein levels of FoxO1 in nuclear fractions of tail muscle, DNA binding by FoxO1 in nuclear extracts increased strongly by $1.9 \pm 0.2$ fold $(P<0.05)$ under anoxia. In the hepatopancreas, despite no change in nuclear FoxO1 protein, and strong increased phosphorylation of S256, the DNA binding activity FoxOl increased strongly by $2.1 \pm 0.3$ fold $(\mathrm{P}<0.05)$ with anoxia. FoxO3 DNA binding activity in nuclear extracts also increased significantly in tail muscle $(1.7 \pm 0.2$ fold; $\mathrm{P}<0.05)$ but no change was seen in FoxO3 DNA binding in hepatopancreas.

\subsubsection{0 cDNA cloning of crayfish MnSOD}

In order to isolate and amplify the cDNA of an un-sequenced target protein, first 'consensus' primers were designed by assessing regions of homology from MnSOD sequences of both vertebrate and invertebrate animals. With a set of multiple forward and reverse primers designed, PCR was carried out for multiple working combinations, using 
$10^{-2} \mathrm{cDNA}$ from $O$. virilis tail muscle and hepatopancreas tissues (both control and stress states. The PCR was run on a temperature gradient (for optimization) with single primer, and tubulin controls to assess the efficacy of the PCR. Following isolation and sequencing of the PCR product, deemed to be at the correct size, 'perfect' primers were designed. From these, a PCR product of $198 \mathrm{bp}$ was retrieved. The product was analyzed by BLAST, and was recognized as a MnSOD type product. FIG. 2.18A shows the partial nucleotide sequence with the corresponding translated 66 amino acid sequence. FIG. 2.18B shows a homology tree produced from the alignment of the partial $O$. virilis $M n S O D$-like protein sequence with MnSOD from red swamp crayfish (Procambarus clarkii), clawed crayfish (Pontastacus leptodactylus), white crayfish (Litopenaeus vannamei), water flea (D. pulex), nematode (C. elegans), mouse (M. musculus) and African clawed frog (Xenopus laevis). The homology tree shows the separation of sequence homology, with the red swamp and clawed crayfish showing the highest homologies (97\% similarity), with the vertebrates (mouse and frog), showing the second highest conservation ( $83 \%$ similarity). However, there appears to be no distinct vertebrate/invertebrate line distinguishing homologies, the water flea and nematode sequences proving to be closer to mammalian sequences (62\% similarity) than to fellow invertebrates (similarity to crustacean group; $30 \%$ ). 0 . virilis showed a $44 \%$ similarity to the top grouping of crustaceans, and $30 \%$ to the remaining sequences. FIG. 2.18C shows a segment of the MnSOD like amino acid sequence that was highly conserved among the crustacean species.

\subsubsection{Transcript expression of $M n S O D$ in response to anoxia}


Changes in the relative transcript levels of $M n S O D$ in response to $20 \mathrm{~h}$ of anoxia were assessed using RT-PCR and the perfect primers designed from the crayfish sequence (FIG 2.19). The representative RT-PCR bands of MnSOD amplicons (shown below the histogram) show, in conjunction with the histogram, the changes in relative transcript levels in response to anoxia. MnSOD transcript levels were normalized against tubulin bands amplified from the same tissue samples. In both tail muscle and hepatopancreas, transcript levels of MnSOD increased significantly by $1.6 \pm 0.04$ and $1.4 \pm 0.03$ fold, respectively (both $P<0.05$ ) under anoxia.

\subsubsection{Protein expression of $M n S O D$ and Catalase in response to anoxia}

The antioxidant enzymes MnSOD and catalase are both transcriptional targets of FoxO transcription factors and so their protein levels were assessed by immunoblotting in response to anoxia in crayfish tissues (FIG 2.20 and 2.21, respectively). Polyclonal $\mathrm{MnSOD}$ and catalase antibodies cross reacted with bands of $\sim 20$ and $60 \mathrm{kDa}$, respectively, which are their expected sizes. Correlated with the effect of anoxia on transcript levels, MnSOD protein levels rose significantly during anoxia by $1.08 \pm 0.01$ and $1.1 \pm 0.02$ fold in tail muscle and hepatopancreas, respectively (both $P<0.05$ ). However, catalase protein content did not change significantly in response to anoxia in either tissue.

\subsubsection{Protein expression of 14-3-3 in response to anoxia}

The 14-3-3 protein is a repressor of FoxO transcription factors, which is attracted to (and subsequently binds) phosphorylated residues on the transcription factor, signaling its nuclear export. Protein levels of 14-3-3 in nuclear protein extracts were assessed by immunoblotting in response to anoxia (FIG 2.22). In both muscle and hepatopancreas, 
nuclear 14-3-3 protein levels significantly decreased under anoxia, dropping by $25 \pm 3 \%$ and $50 \pm 9 \%$, respectively (both $\mathrm{P}<0.05$ ).

\subsection{Discussion}

Akt or protein kinase B is the main effector of the insulin/IGF pathway of signaling. In its role as the hub of downstream signaling, Akt promotes pro-developmental and prosurvival pathways. In the context of a stress adapted model, such as the anoxia resistant crayfish $(O$. virilis), this provides a contradictory response. While the pro-survival/antiapoptotic aspect of Akt signaling would be critical to long term survival throughout an extended anoxia/hypoxia excursion, other pathways which Akt positively influences (e.g. glycogen and protein synthesis) are energetically expensive and as such would be incongruous to the general response of metabolic rate depression that is seen in the crayfish. Furthermore, the activity of FoxO transcription factors, with both pro-apoptosis and pro-survival (via antioxidants) transcriptional targets, also creates a dichotomy. As an overview, studying the regulation of Akt signaling and in particular its effects on FoxO transcriptional activity, is an exercise in compromise. Hence the while some specific targets do not correlate positively, the trends within the data (Table 2.3) are largely in accordance with the initial hypothesis.

\section{Regulation of Akt/FoxO signaling in hypometabolism}

In C. elegans, the main cellular switch to dauer entry is suppression of the insulin response pathway; particularly signaled via Akt (see Appendix A) (Vanfleteren and Braeckman, 1999). Dauer signaling is mediated through a series of 'abnormal dauer 
formation' (DAF) genes, of which the FoxO ortholog (DAF-16), is critical. Through repression of Akt activity, primarily prevention of Akt phosphorylation by upstream elements, DAF-16 transactivates a number of dauer promoting gene targets and the nematode enters its period of hypometabolism (Paradis et al., 2009). As in higher animal models, the FoxO transcription factors (DAF-16a and b), target genes that promote cell cycle attenuation and cell preservation (e.g. antioxidants) (Burgering and Kops, 2002). What sets C. elegans aside from many animal systems of metabolic rate depression is the severity of the effects on the animal's lifespan. The dauer period is often referred to as a period of agelessness, in regards to the lack of development within the period of hypometabolism. Part of this is due to stringent control of the cell cycle, and its attenuation throughout the dauer period that sees entrance to a 'quiescent' or nondividing phase (Van den Heuvel, 2005).

In the anoxia resistant crayfish, not only is the period of hypometabolism significantly shorter (in regards to total organism lifespan), but it is also seasonal. With a lifespan of around 3 years (Crocker and Barr, 1968), the crayfish, hypothetically, could undergo this hypometabolic period multiple times, and as such, the response to the environmental stress is less severe. Development during this time is not attenuated, and there is no evidence to suggest that 'agelessness' through cell cycle quiescence occurs. Despite this, crayfish metabolism is reduced, and at the end of periods of severe hypoxia and/or anoxia the animals do not appear to suffer significant tissue damage through apoptosis; which may be signaled in part by oxidative damage with a return to higher oxygen levels. In regards to this last point, ROS damage following a period of severe (hypoxic/anoxic) oxygen stress is well documented (Hermes-Lima et al., 1998). With the 
resumption of regular oxygen levels the electron transport chain (ETC) is often unable to respond to the rapidly increased oxygen levels. Oxygen, which is reduced to water, can in this case be incompletely reduced, resulting in superoxide $\left(\mathrm{O}_{2}^{-}\right)$production; particularly at complexes I and III of the ETC. The superoxide dismutase family (SOD) converts superoxide into hydrogen peroxide $\left(\mathrm{H}_{2} \mathrm{O}_{2}\right)$, which can subsequently be converted into water and oxygen by catalase. Both of these antioxidants are targets of FoxO transactivation, presenting a pro-survival aspect to its signaling. Hence it is not expected that a unilateral form of Akt/FoxO regulation be enforced in the crayfish, like the nematode, in response to anoxia.

Akt expression and activation in response to anoxia

Canonical insulin/IGF signaling involves Akt activation mediated by PDK1 phosphorylation of the threonine 308 residue on Akt (Alessi et al., 1996). PDK1 itself must be phosphorylated in order to phosphorylate and activate Akt. The serine 241 residue of PDK1 is critical to its activity. Unlike other phosphorylation sites on PDK1, it is protected from dephosphorylation by protein phosphatase $2 \mathrm{a}(\mathrm{PP} 2 \mathrm{a})$, and it is also suspected that PDK1 can autophosphorylate and activate itself at this residue in conjunction with standard activation through PIP3 (Casamayor et al., 1999). Previous studies of land snail estivation (another form of hypometabolism), showed that Akt phosphorylation on serine 473 , a residue phosphorylated by the mammalian target of rapamycin (mTOR), increased in response to limited oxygen (Ramnanan et al., 2007). In the anoxia resistant crayfish, however, both Akt protein levels and phosphorylation on threonine 308 increased significantly in both tail muscle and hepatopancreas in response 
to anoxia. In tail muscle during anoxia this was also correlated with increased phosphorylation of PDK1 (serine 241) indicating canonical P13K signaling.

Although it is commonly understood that Akt phosphorylation occurs at the cell membrane in accordance with its role in insulin/igf receptor mediated signaling, there is also an avenue for nuclear phospho-Akt activity. The majority of Akt targets are located in the cytoplasm, but in the case of the FoxO transcription factors, Akt is able to translocate to the nucleus in order to phosphorylate these proteins and prevent their binding to DNA (Trotman et al., 2006). This is in accordance with pro-oncogenic roles of Akt seen in many cancers. The primary regulating factor is of this event is the PTEN phosphatase which is classified as a tumor suppressor protein; its absence allows Akt activity and subsequent pro-proliferation, anti-apoptosis responses (Trotman et al., 2006). In $O$. virilis, there were no significant changes in levels of phosphorylated Akt in the nucleus during anoxia, indicating that despite its increased presence in the cytoplasm, active Akt is not necessarily given an increased nuclear role under anoxia. A future avenue of research may be in the study of the PML tumor suppressor which recruits PP2a into the nucleus to dephosphorylate Akt (Trotman et al., 2006).

The phosphatase PTEN, as mentioned above, is frequently referred to as a tumor repressor protein both in respect to its regulatory role on proto-oncogene $\mathrm{Akt}$, and in its common absence in many types of cancer (Jiang and Liu, 2008). In C. elegans its ortholog (DAF-18) is similarly critical to Akt suppression, which is essential not only in dauer entry, but also in L1 phase larval diapause (Fukuyama et al., 2006). The role of PTEN in the cytoplasm has long been known, but its nuclear role has only recently been elucidated. In keeping with its role as a tumor suppressor, nuclear PTEN is seen to 
interact with anaphase promoting complex/cyclosome (APC/C), which is a cell cycle regulator (Song et al., 2011). APC/C is an E3 ubiquitin ligase that promotes the degradation of critical cell cycle machinery (including mitotic cyclins and mitotic kinases) in order to attenuate the cell cycle and subsequent excessive proliferation. It was discovered that nuclear PTEN promotes interaction of cadherin-1 (CHD1) with the APC/C complex, which enhances APC/C-CDH1 activity, and their tumor suppression capacity (Song et al., 2011). In O. virilis, the static levels of PTEN in the cytoplasm in conjunction with significant increases in PTEN protein in the nucleus may be indicative of this uncoupled behavior of PTEN in the cell. Certainly, the unchanged level of cytoplasmic PTEN correlates with the increase in PDK1 and Akt phosphorylation, under anoxia, and while there is no apparent cell cycle quiescence in the crayfish, cellular proliferation rates may be reduced in conjunction with this increase in nuclear PTEN. Regulation of GSK3 in $O$. virilis in response to anoxia Glycogen synthase kinase (GSK3) is represented by two isoforms, $\alpha$ and $\beta$. The two forms are highly conserved, and although there are suggestions that, at least in mammalian tissues, there are patterns of differential expression between the two forms, mRNA and protein levels do not necessarily support this theory (Ali et al., 2001). The primary form of GSK3 regulation stems from Akt, which phosphorylates GSK3a at serine 21. Following Akt's role in pro-survival signaling, inhibition of GSK3 prevents a number of cell cycle attenuation/apoptosis signals. GSK3 is able to attenuate the cell cycle in different ways. Firstly, by phosphorylating cyclin D, the cyclin is unable to complex with cyclin dependant kinases and form the necessary cell cycle progression $\left(\mathrm{G}_{1} / \mathrm{S}\right)$ complex (Liang and Slingerland, 2003). Secondarily, GSK3 is an inhibitor of the 
proto-oncogene c-myc. Through inhibition of c-myc, GSK3 allows production of cell cycle inhibitors (p15, p21 and p27) which c-myc is known to suppress (Liang and Slingerland, 2003; Gartel and Shchors, 2003). While this is undoubtedly important to cellular survival, negative regulation of GSK3 would also support a number of energetically expensive procedures, such as glycogen and protein synthesis. As such, like regulation of FoxOs, there are compromises to be made in a model of metabolic rate depression. In the crayfish under anoxia protein levels of GSK3a increased in the muscle and phosphorylation of serine 21 rose in both tissues. This is a profile of GSK3a inhibition and would certainly suggest a pro-survival signal that correlates with the profile of increased Akt activity that is also seen under anoxia.

The roles and regulation of FoxO transcription factors in the anoxia resistant crayfish The FoxO transcription factors have long been associated with stress response, both in systems of metabolic rate depression (Mukhopadhyay, 2006) and in clinical states such as a cancer (Maiese et al., 2009). The FoxOs are often seen as critical downstream targets of insulin/IGF/Akt signaling. FoxOs can also be targets of SGK phosphorylation. This activity, which occurs in parallel to stimulation of the canonical insulin/IGF pathway, is also seen in C. elegans (see Appendix A and B.2). In O. virilis under anoxia, increases in SGK protein levels were seen in both tissues, however increased phosphorylation (conferring activity) occurred only in the tail muscle (hepatopancreas levels of phosphorylation remained constant). Functionally, this correlates with the Akt activity pattern already seen and is further indicative of pro-survival and developmental signaling during anoxia. 
Total FoxO1 protein levels were static in crayfish tail muscle under anoxia, although that manifested as an increase in the cytoplasmic fraction and a decrease in the nuclear fraction. Correlated with this, phosphorylation of serine 256 (a specific Akt phosphorylation target) increased in the cytoplasmic fraction and decreased in the nucleus, suggesting that the ratio of phosphorylation may be linked to protein levels. In the hepatopancreas, cytoplasmic FoxO1 protein levels increased, while nuclear protein levels stayed static. Interestingly, nuclear phosphorylation unrelated to general protein levels, was seen in the hepatopancreas, while phosphorylation levels did not increase under anoxia in the cytoplasm despite increased FoxO1 levels. While these results did not indicate increased nuclear activity, FoxO1 DNA binding activity was surprisingly strongly increased in both tissues in response to anoxia. This suggests a mechanism of enhancing FoxOl transcriptional activity that is not related to protein level or phosphorylation state.

FoxO3 protein levels in the cytoplasmic fraction of tail muscle decreased, whereas there was no change in phosphorylation of serine 253 (a specific Akt and SGK1 phosphorylation target). In the nuclear fractions, FoxO3 protein levels increase in conjunction with increased phosphorylation, under anoxia. This corresponds with the increased active SGK1 in the tail muscle under anoxia. In the hepatopancreas, protein levels in both cytoplasmic and nuclear fractions were unchanged by anoxia. Phosphorylation levels were similarly unchanged in the cytoplasmic fraction, but significantly decreased in the nucleus, providing a potential for FoxO3 activity. Despite this, FoxO3 DNA binding increased in the muscle. 
What is interesting is that despite a relatively unambiguous trend of reduced FoxO activity, through generally lowered nuclear FoxO presence and increased phosphorylation under anoxic conditions, DNA binding activity increased significantly. This overall trend suggests that despite what appears to be a canonical inactivation of FoxOs through increased Akt and SGK activity under anoxia, there is still residual FoxO transcriptional activity. One possible reason for this is may be that it has been noted that the transcriptional activities of FoxOs are susceptible to other forms of post-translational modification (e.g. acetylation, methylation and ubiquitination) (Calnan and Brunet, 2001), which may be influencing the DNA binding in existing nuclear FoxOs during anoxia. Furthermore, decreased nuclear 14-3-3 content was seen in both tissues under anoxia. A reduced nuclear presence of 14-3-3 would indicate reduced expulsion of phospho-FoxO, and thereby allow either limited (depending on the site phosphorylated) DNA binding or a greater potential for nuclear dephosphorylation. Both of these could allow sustained and/or enhanced FoxO transcriptional activity under anoxia. The role of FoxOs in antioxidant defense and its importance in a model of anoxia resistance

Amongst the downstream targets of FoxOs are two antioxidant target genes, catalase and MnSOD. Although catalase protein levels did not increase in crayfish tissues under anoxia, MnSOD levels showed an interesting response. Both transcript and protein levels of MnSOD increased in both crayfish tissues under anoxia. This suggests that despite a largely inhibitory profile, FoxOs still retain transcriptional capacity under anoxia. The capacity of the FoxOs to mediate responses to oxidative stress is well described (Storz, 2011; Goto and Takano, 2009; Huang and Tindall, 2007). In many 
cases the FoxO response to oxidative stress seems to be to initiate cell death, which correlates with their suppression in tumors exhibiting oxidative stress (as a response to reperfusion) (Storz, 2011), their role in a system adapted to oxygen limitation and oxidative stress may be one of preparation. The elevation of MnSOD in both tail muscle and hepatopancreas during anoxia (a situation in which there is little or no oxidative stress), appears to be a preparative adaptation for the return to high $\mathrm{O}_{2}$ levels after their winter imposed anoxia. Mitochondrial induced ROS release (described above) is a common occurrence in ischemia/reperfusion during which increased oxygen overwhelms the electron transport chain culminating in large amounts of incompletely reduced oxygen (Hermes-Lima et al, 1998). Hence the profile of increased FoxO DNA binding, in conjunction with increased transcript and protein levels of MnSOD indicate a potentially important role for the FoxO proteins in enhancing antioxidant defense in support of the anoxia resistance of the crayfish.

\section{Conclusions}

The regulation of Akt signaling in the anoxia resistant crayfish, although at times seemingly contradictory to the principles of metabolic rate depression, are indicative of adaptive changes that support cellular survival throughout the anoxic period. The data also point to a distinctly 'un-dauer like' response (see Appendix A and Table 2.3), but nonetheless propagates pro-survival signals that would be essential during the metabolic challenge.

Through suppression of the activity of targets capable of cell cycle attenuation and apoptosis stimulation, active Akt under anoxia in $O$. virilis contributes to extending cellular viability in the energy-limited anoxic state. Despite this, the downstream 
transcription factors from the forkhead box family seem to still be able to exert transcriptional capacity. While the pro-apoptotic aspect of FoxO activity is contrary to the cell survival needs of the anoxic crayfish, increased Akt activity appears to largely minimize FoxO activity, seen through phosphorylation and cytoplasmic accumulation of the transcription factors. Nonetheless, increases in selected antioxidant targets (e.g. MnSOD) are suggestive of a protective role for the FoxOs despite their general repression. 


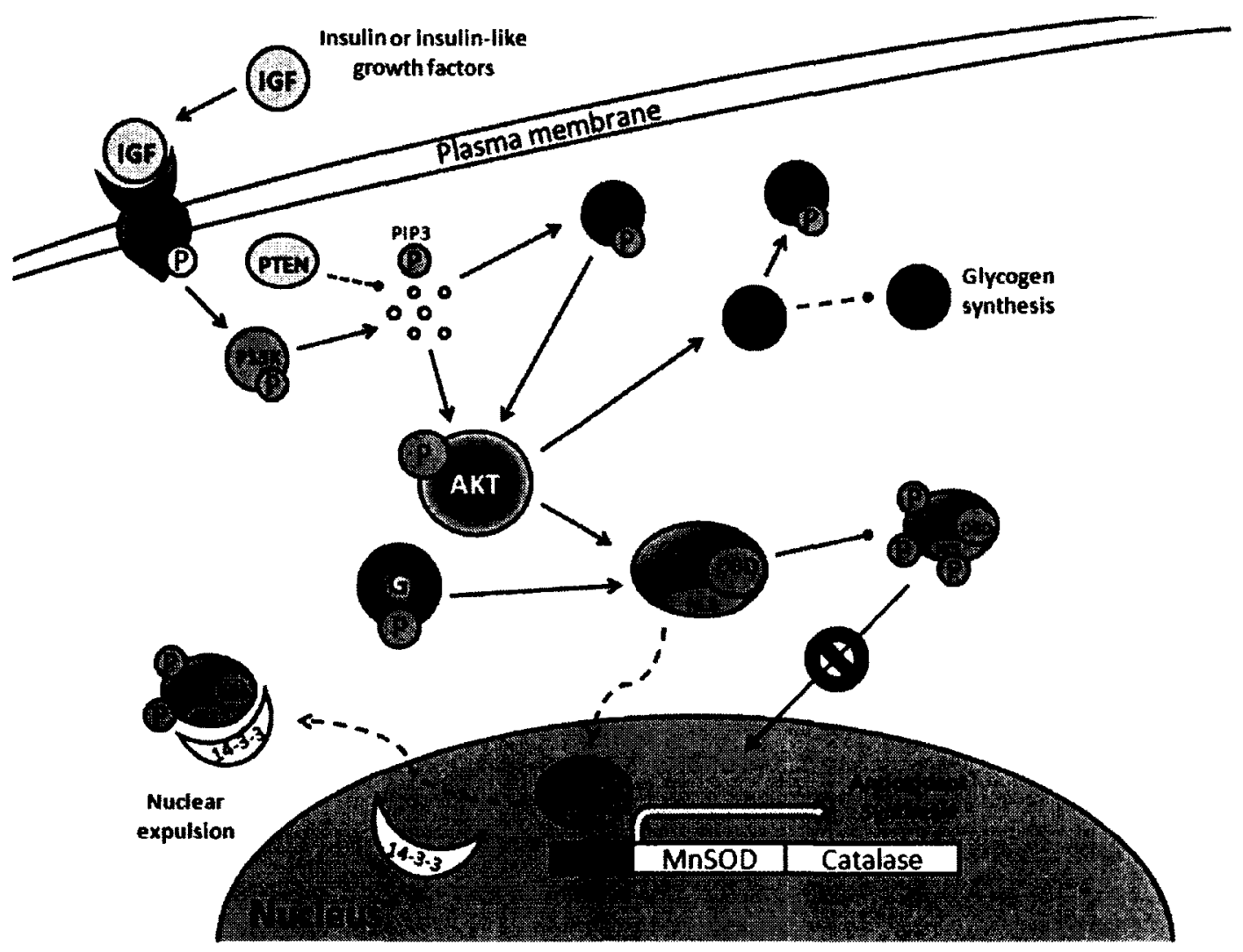

Figure 2.1: An overview of the Akt pathway. The major protein kinase B (Akt) is activated by upstream canonical insulin/insulin like growth factor signaling. Through PIP3 and/or PDK-1, Akt is phosphorylated and activated. Akt is central to numerous cellular processes such as cell cycle regulation and apoptosis control, and affects glycogen synthesis and antioxidant production through the repression of GSK $3 a$ and FoxO transcription factors. The FoxO transcription factors, when phosphorylated are unable to enter the nucleus and remain anchored in the cytoplasm. Phosphorylation state of Akt, via the PTEN phosphatase also controls pathway activity. 


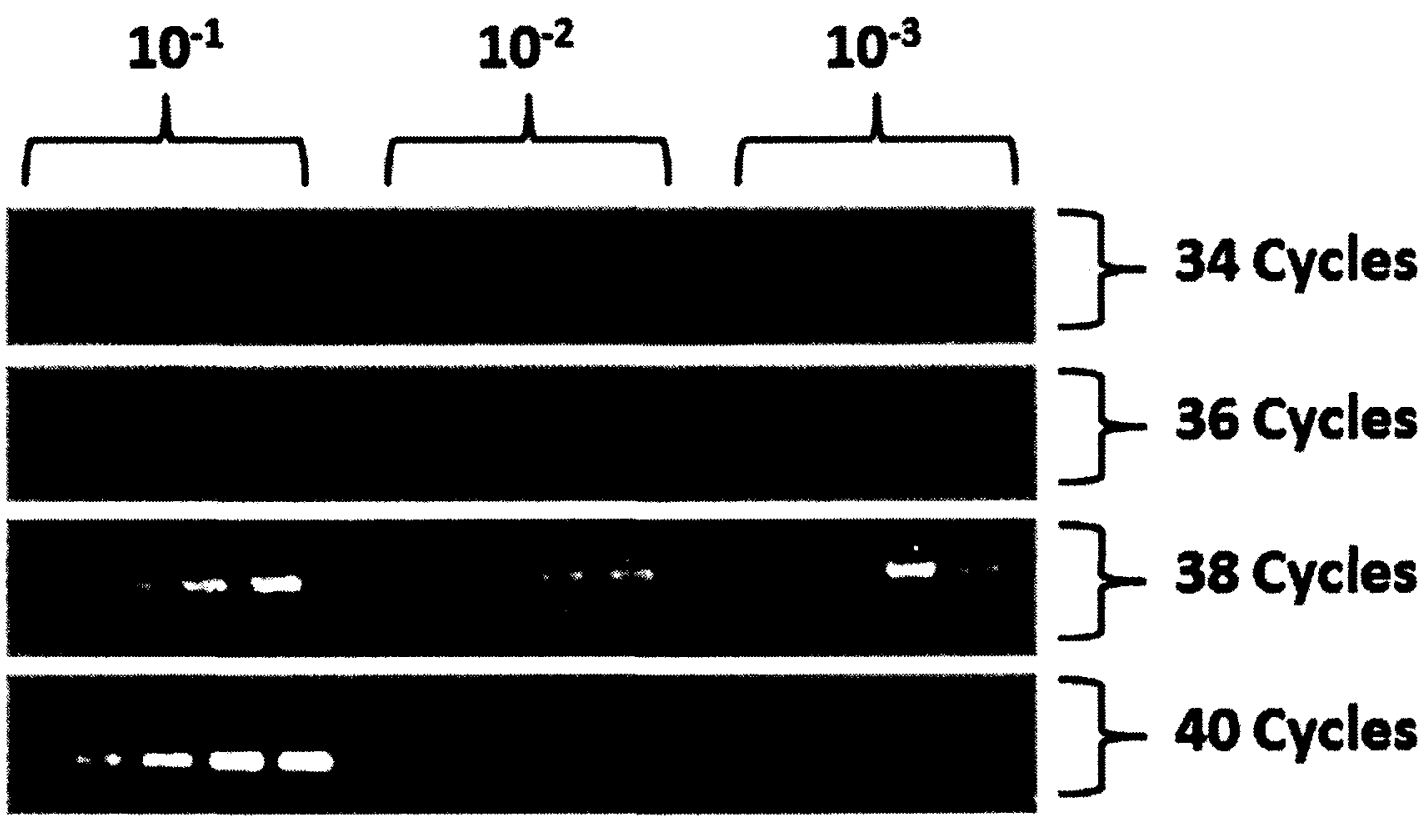

Figure 2.2: Determination of optimum PCR cycle count by analysis of signal saturation at multiple concentrations. Tubulin bands from $10^{-1}$ down to $10^{-3}$ dilution are seen from 34 to 40 cycles, with the optimal signal measurement seen at 38 cycles.

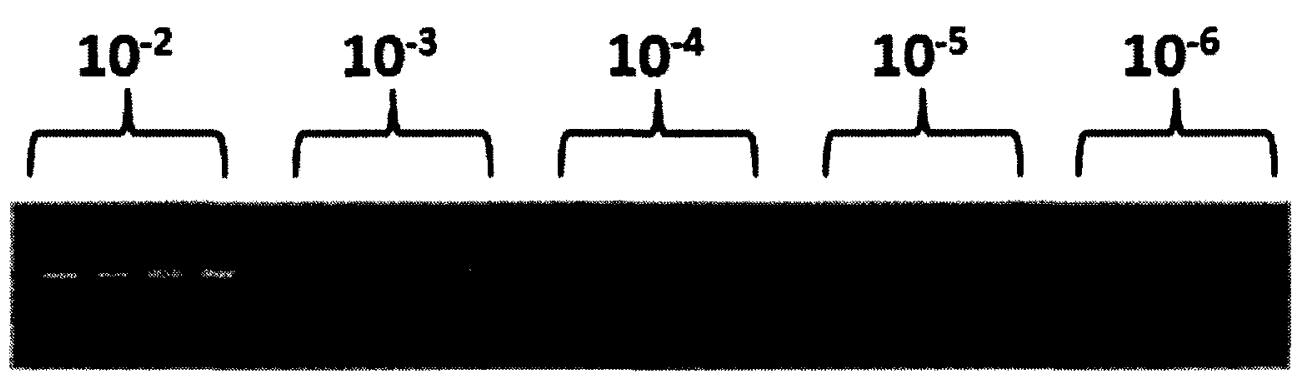

40 cycles, tubulin $53^{\circ} \mathrm{C}$ (faint bands at $10^{-4}$ )

Figure 2.3: Tubulin concentration gradient showing measurable signal with increasing dilution. Tubulin bands ranging from $10^{-2}$ to $10^{-6}$ concentration, amplified over 40 cycles to ensure maximal (saturated) signal, indicates $10^{-3}$ to be the lowest usable concentration. 


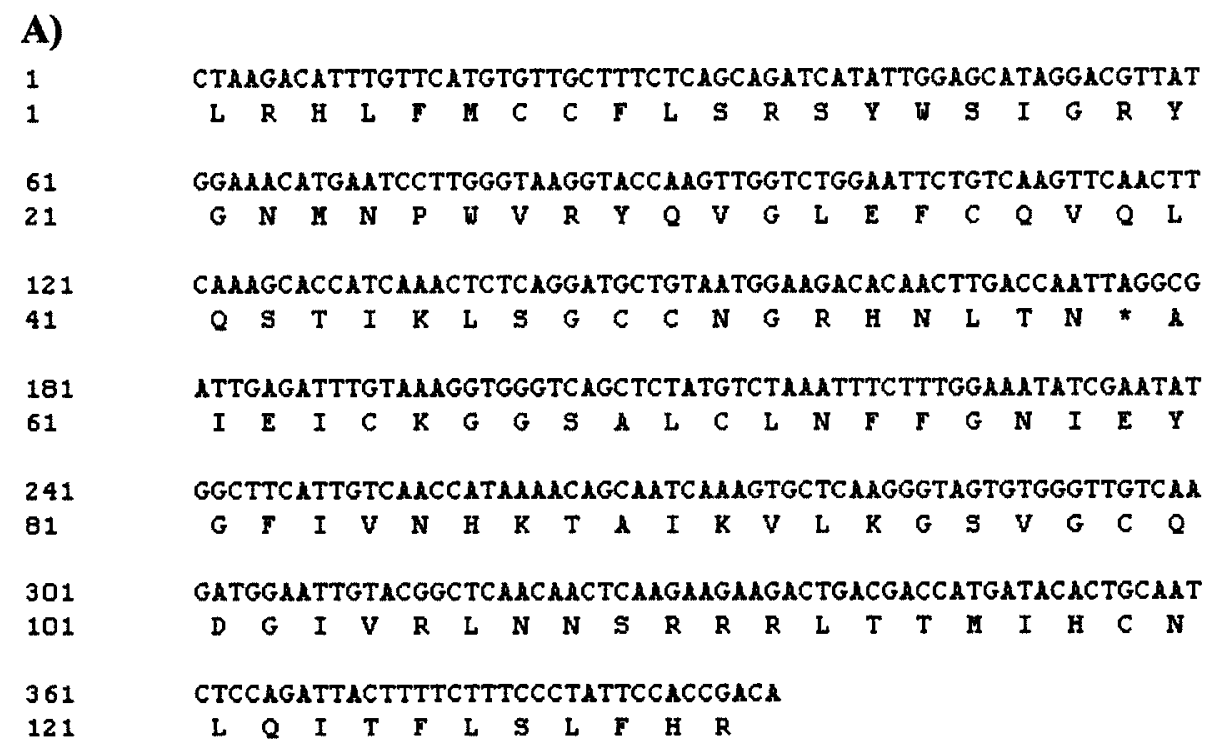

B) American Lobster:Crayfish final identity $=438$

239 CTGGTGTGTACCGTCAGCTGTTCCATCCTGAGCAGTTGATCACTG. GCAAGGAAGATGCG

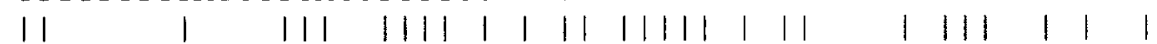

1 CTAAGACATTTGTTCATGTGTTGCTTTCTCAGCAGATCATATTGGAGCATAGGACGTTAT

298 GCAAACAATTATGCACGTGGTCATTATACAATTGGAAAGGAAATTGTTGACATTGTACTT

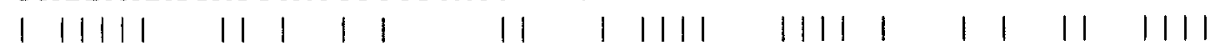
61 GGAAACATGAATCCTTGGGTAAGGTACCAAGTTGGTCTGGAATTCTGTCAAGTTCAACTT

358 GACCGCATCCGCAAACTGGCAGACAACTGCGCTGGTCTTCAGGGATTCCTAATTTTCCAT

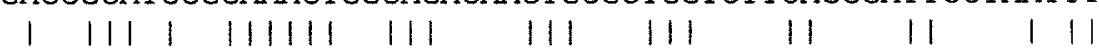

121 CAAAGCACCATCAAACTCTCAGGATGCTGTAATGGAAGACACAACTTGACCAATTAGGCG

418 TCCTTTGGCGGTGGCACTGGCTCAGGATTTACTTCTTTATTGATGGAAAGGCTTTCTGTT

181 ATTGAGATTTGTAAAGGTGGGTCAGCTCTATGTCTAAATTTCTTTGGAAATATCGAATAT

478 GACTATGGAAAGAAGAGCAAGCTAGAATTTGCAATTTACCCAGCTCCCCAGGTAGCTACT

241 GGCTTCATTGTCAACCATAAAACAGC

C)

$100 \% 90 \% 80 \% \quad 70 \% \quad 60 \% \quad 50 \% \quad 40 \% \quad 30 \%$

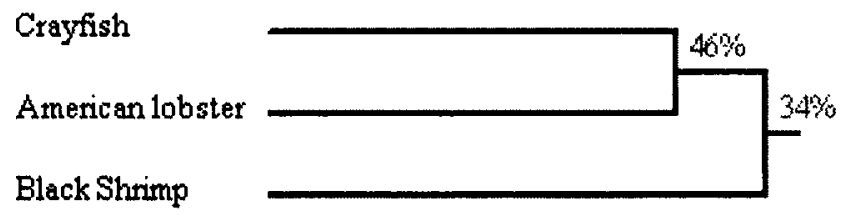


D)

i)

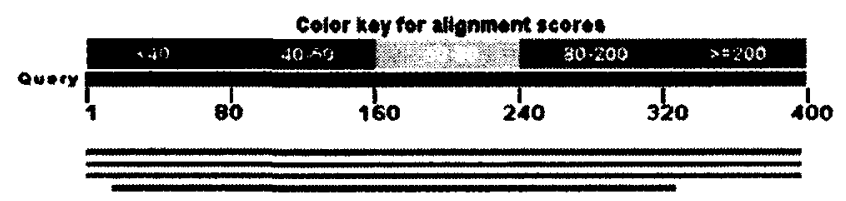

ii)

\begin{tabular}{|c|c|c|c|c|c|c|}
\hline $45 \leqslant 2.28 .4$ & Homarus americanus alpha-Il tubulin mRNA, complete cds & 394 & 394 & $99 \%$ & 2E. 106 & $82 \%$ \\
\hline Ya18.0.1 & Homarus americanus atpha-1 tubulin mRNA, complete cds & 304 & 304 & $99 \%$ & 3e-79 & $77 \%$ \\
\hline $54-522+2$ & PREOICTED: Apis mellifera tubulin alpha-1 chain-like (LOC550327), $\mathrm{mR}$ & $38 *$ & 282 & 994. & $16-32$ & 732 \\
\hline Av22505201 & Aurelia aurite alpha-tubulin mRMA, partial cos & 233 & 273 & 7846 & $80-30$ & $79 \%$ \\
\hline
\end{tabular}

E)

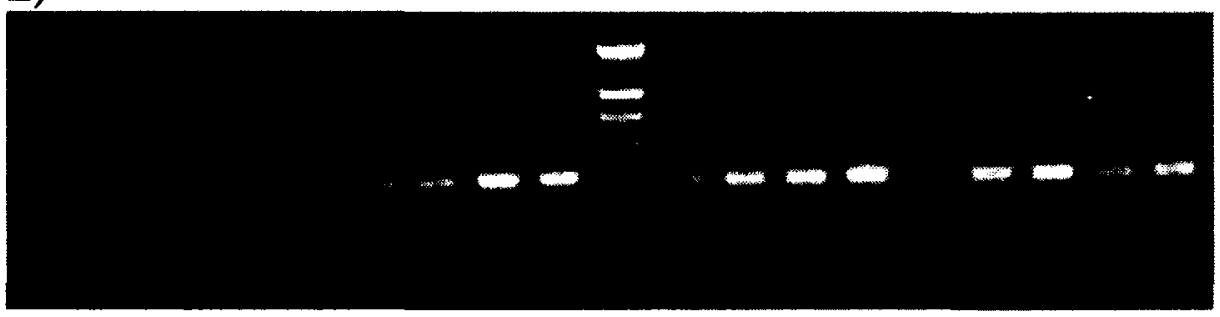

Figure 2.4: Partial nucleotide and deduced amino acid sequence of $O$. virilis alpha tubulin II protein.

(A) The partial cDNA sequence of a tubulin like protein, with the corresponding amino acid sequence is shown. Nucleotides and amino acids are numbered on the left. The nucleotide sequence was 395 nucleotides in length, and encoded 130amino acids.

(B) Sequence alignment of a segment of the tubulin-like $O$. virilis PCR product in comparison to another crustacean model, the American lobster (Homarus americanus)

(C) Homology tree of cDNA sequences produced from an alignment of the partial $O$. virilis tubulin like protein with tubulin from the black tiger shrimp (Penaeus monodon) and American lobster (Homarus americanus). Accession numbers are AAM73792 for shrimp, and AAC47305 for lobster. The percentage values correspond to the shared identity among the corresponding species.

(D) Top cDNA sequence identity matches, ascertained from BLAST, indicating three separate invertebrates as the top similarity results; American lobster (Homarus americanus), honey bee (Apis mellifera) and moon jelly (Aurelia aurita). I) is the graphical representation of the strength of the query, and ID) are the corresponding matches within the BLAST database. 
(E) Sample amplicons (and position relative to a DNA ladder) of $O$. virilis tubulin. From left, $\mathrm{n}=4$, Control tail muscle cDNA, 20h anoxic tail muscle cDNA, control hepatopancreas cDNA and $20 \mathrm{~h}$ anoxic hepatopancreas cDNA. Tubulin transcript levels showed no significant changes between control and anoxia in either tissue. 
Figure 2.5

A)

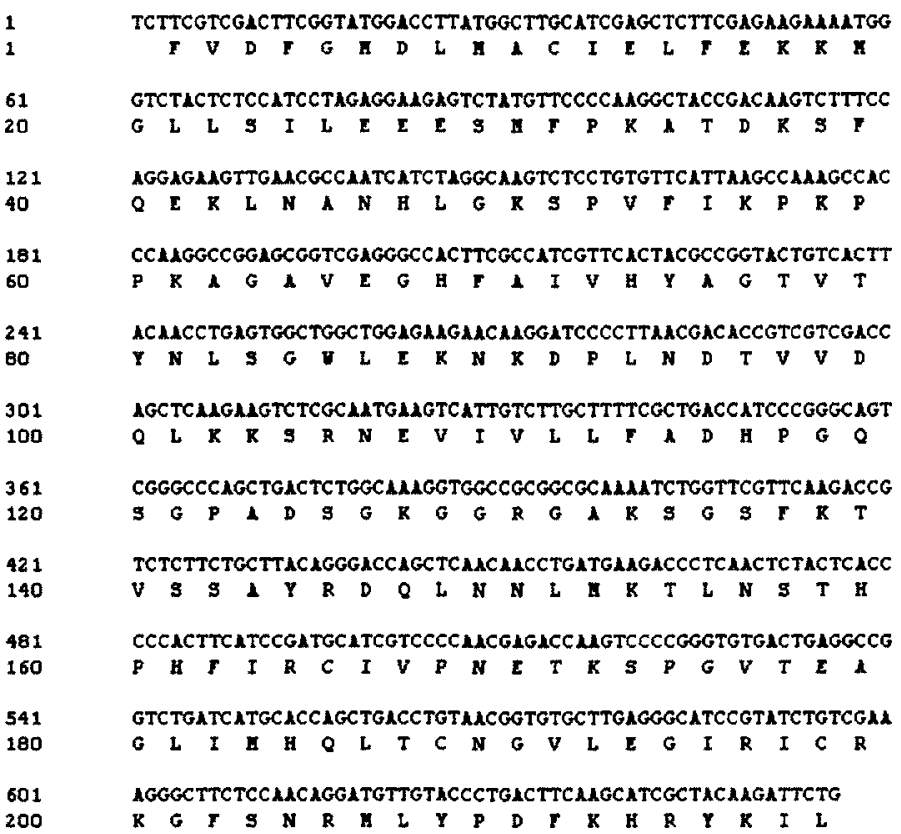

B)

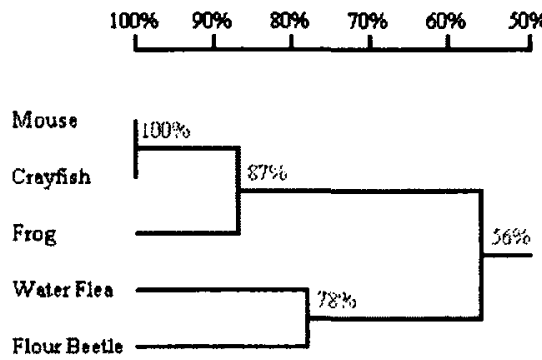

C)

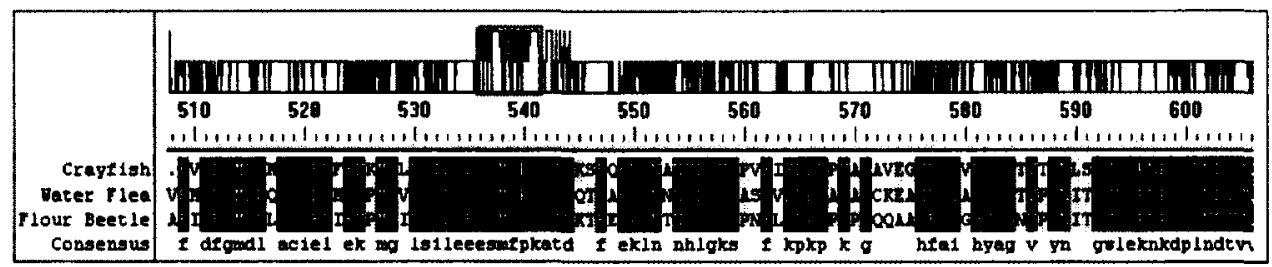


D)

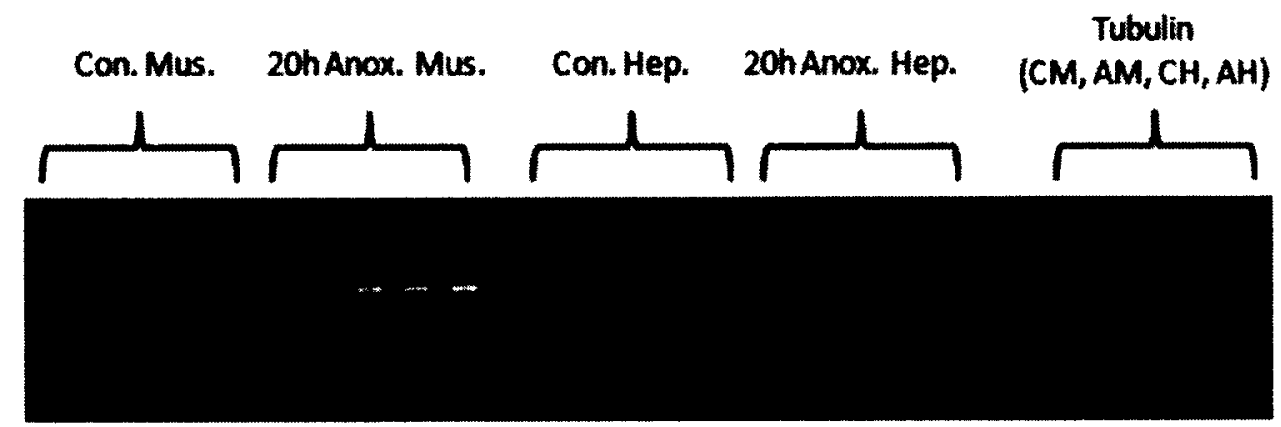

Figure 2.5: Partial nucleotide and deduced amino acid sequence of $\boldsymbol{O}$. virilis myosin heavy chain protein.

(A) The partial cDNA sequence of a myosin heavy chain protein, with the corresponding amino acid sequence is shown. Nucleotides and amino acids are numbered on the left. The nucleotide sequence was 598 nucleotides in length, and encoded 199 amino acids.

(B) Homology tree produced from an alignment of the partial $O$. virilis myosin heavy chain protein sequence with myosin heavy chain from Mouse (Mus musculus), African clawed frog (Xenopus laevis), Water flea (Daphnia pulex) and Flour beetle (Tribolium castaneum). Accession numbers are AAA39795 for mouse, AAA49908 for frog, EFX87106 for water flea, and XP_001813815 for flour beetle. The percentage values correspond to the shared identity among the corresponding species.

(C) Sequence alignment of a segment of the myosin heavy chain translated protein sequence from $O$. virilis in comparison to other arthropods (See section B for accession numbers).

(D) Sample amplicons and bp position relative to tubulin (CM, 20h AM, CH, 20h AH) for $O$. virilis myosin heavy chain transcripts. From left, $\mathrm{n}=4$, Control tail muscle cDNA, 20h anoxic tail muscle cDNA, control hepatopancreas cDNA and 20h anoxic hepatopancreas cDNA. Transcript levels of $m h c$ exhibited no significant changes between aerobic and anoxic states in either tissue. 


\section{Control 20h Anoxia}

1
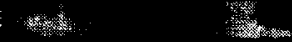

2

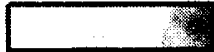

3

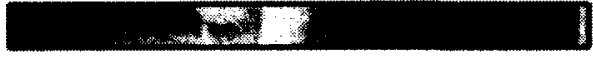

4
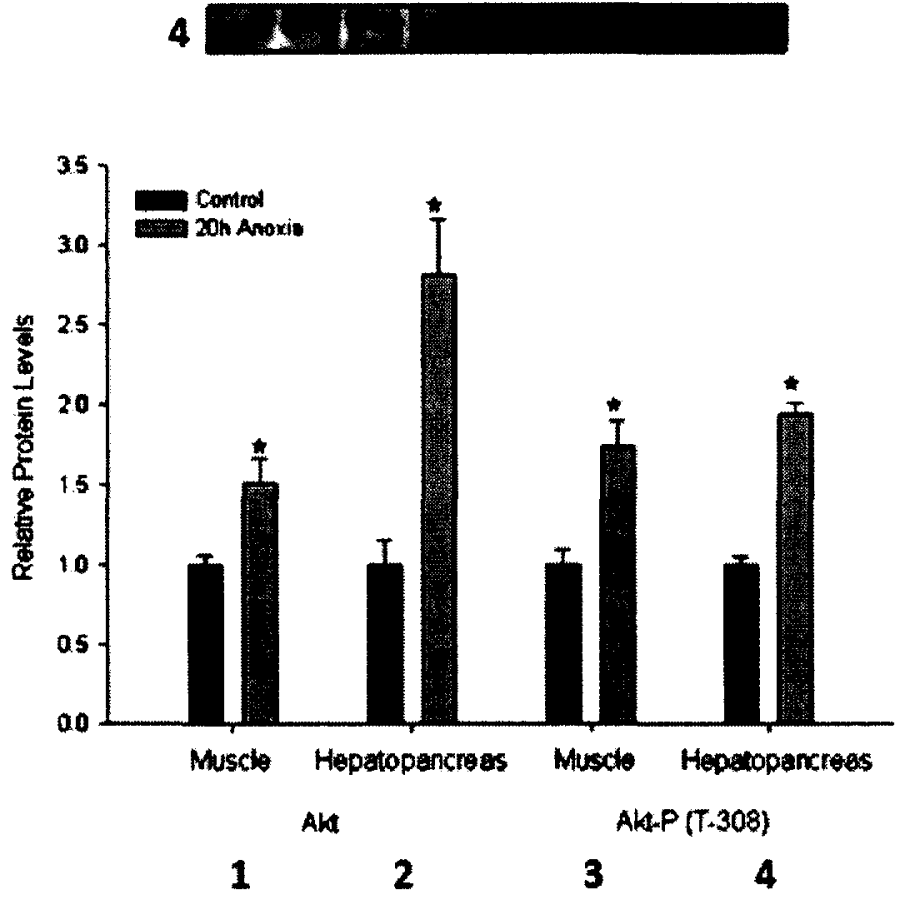

Figure 2.6: Effect of $20 \mathrm{~h}$ anoxia exposure on Akt protein levels and its phosphorylation (at $\mathrm{Thr} 308$ ) in $O$. virilis tissues.

Changes in total Akt protein content in crayfish tissues in response to anoxia, as determined by western immunoblotting, are seen on the left side. Changes in phosphorylation state of the threonine 308 residue are seen on the right side. Data are means \pm SEM, $n=4$ independent trials for muscle and hepatopancreas. * indicates significantly different from the corresponding aerobic control, $P<0.05$. 


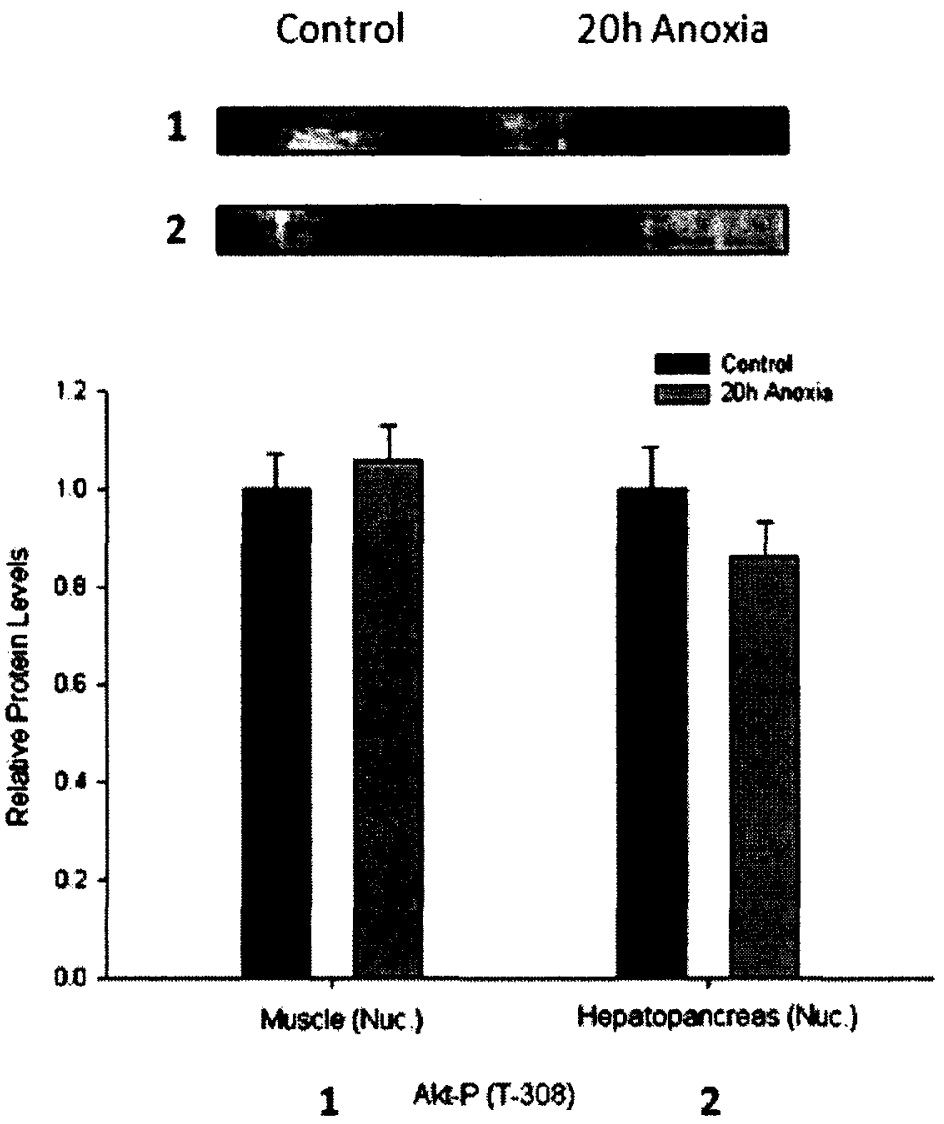

Figure 2.7: Effect of $20 \mathrm{~h}$ anoxia exposure on nuclear expression of Akt phosphorylation (T308) in $O$. virilis tissues.

Relative amounts of phospho-Akt (Thr 308) in nuclear extracts of crayfish tissues in response to anoxia, as determined by western immunoblotting.

Data are means $\pm S E M, n=4$ independent trials for muscle and hepatopancreas. * indicates significantly different from the corresponding control, $P<0.05$. 


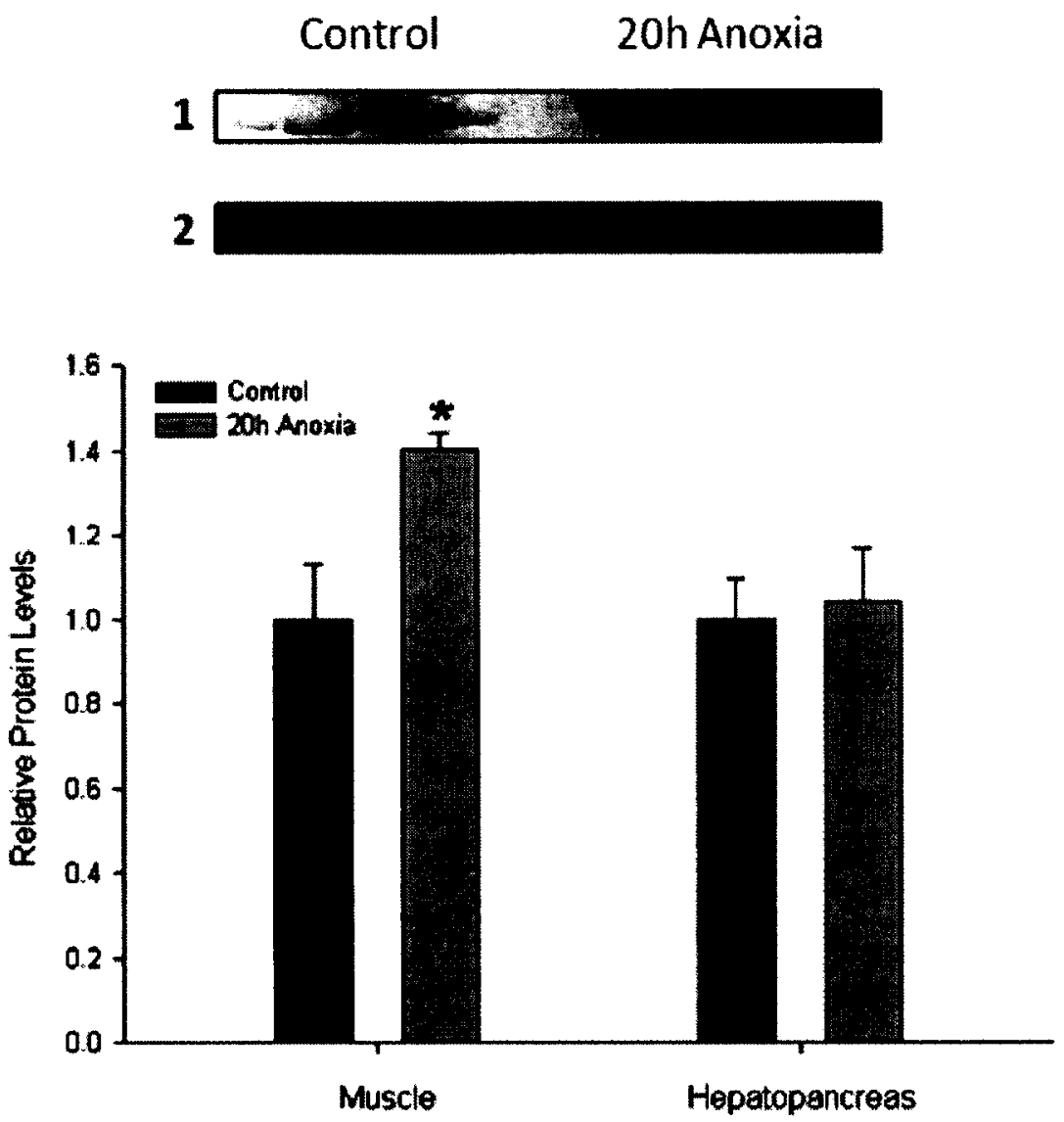

PDK1-P (S-241)

1

2

Figure 2.8: Effect of $20 \mathrm{~h}$ anoxia exposure on phosphorylation of PDK1 (S241) in $O$. virilis tissues.

Changes in relative phosphorylation of PDK1 at serine 241 in total soluble extracts of crayfish tissues in response to anoxia, as determined by western immunoblotting. Data are means $\pm S E M, n=4$ independent trials for muscle and hepatopancreas. * indicates significantly different from the corresponding control, $P<0.05$. 


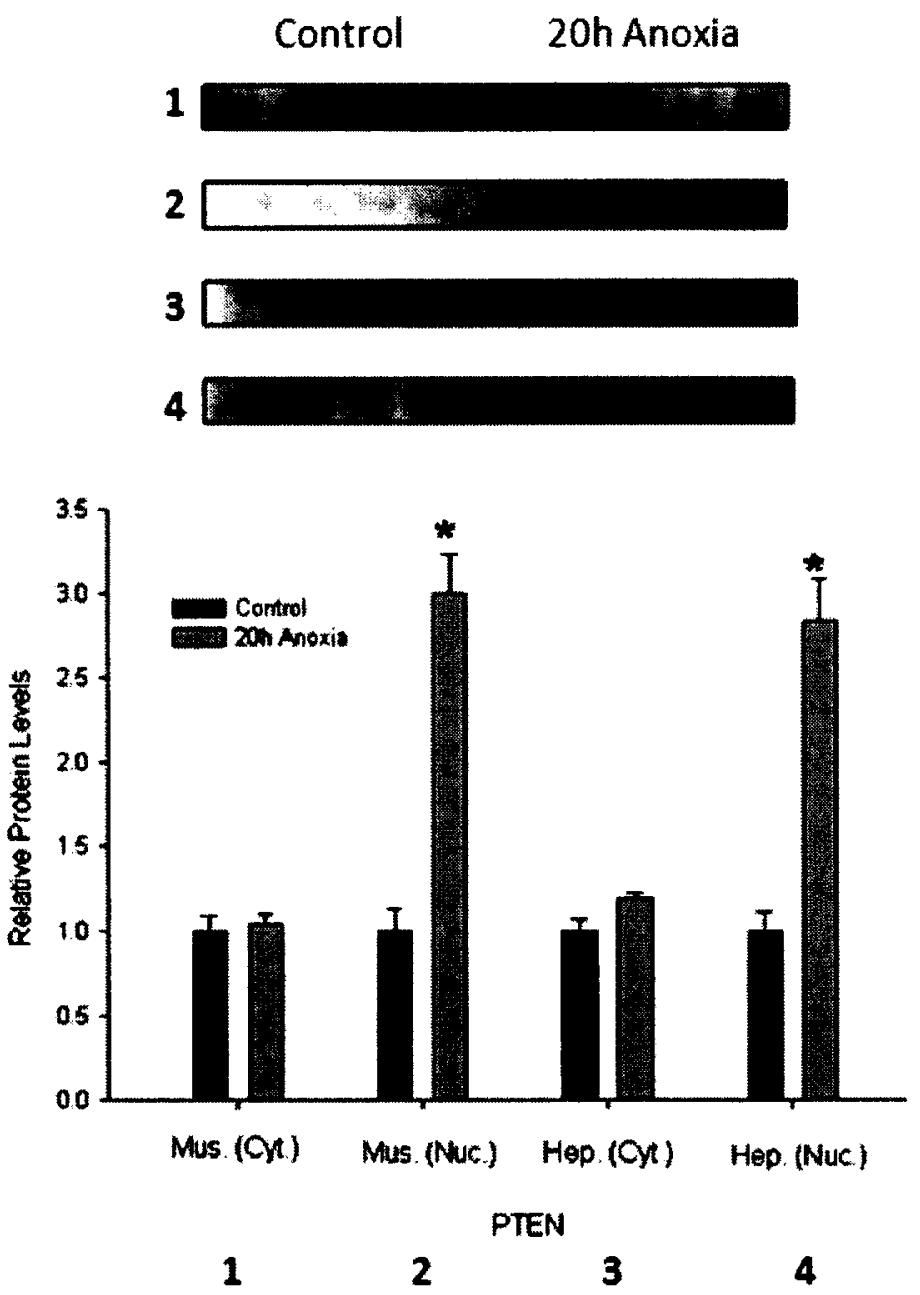

Figure 2.9: Effect of $20 \mathrm{~h}$ anoxia exposure on the subcellular distribution of PTEN in 0 . virilis tissues.

Relative protein levels of PTEN phosphatase in cytoplasmic and nuclear fractions of crayfish tissues (tail muscle, left; hepatopancreas, right) in response to anoxia, as determined by western immunoblotting.

Data are means \pm SEM, $n=4$ independent trials for muscle and hepatopancreas. * indicates significantly different from the corresponding control, $P<0.05$. 

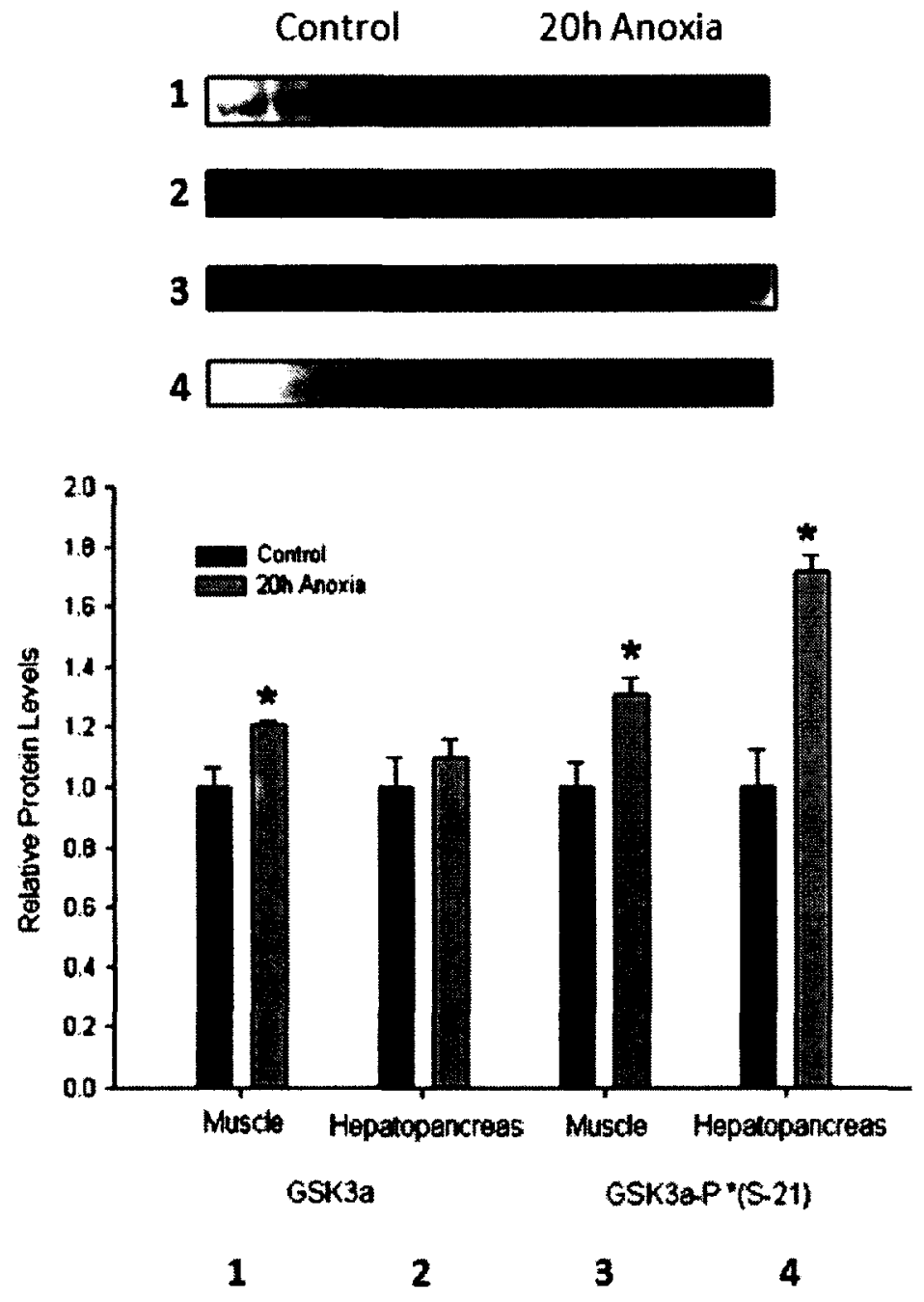

Figure 2.10: Effect of $20 \mathrm{~h}$ anoxia exposure on GSK3a protein levels and activation in $O$. virilis tissues.

Changes in GSK3a protein levels and phosphorylation of GSK3a on serine 21 in soluble protein extracts of crayfish tissues in response to anoxia, as determined by western immunoblotting.

Data are means $\pm S E M, n=4$ independent trials for muscle and hepatopancreas. ${ }^{*}$ indicates significantly different from the corresponding control, $P<0.05$. 

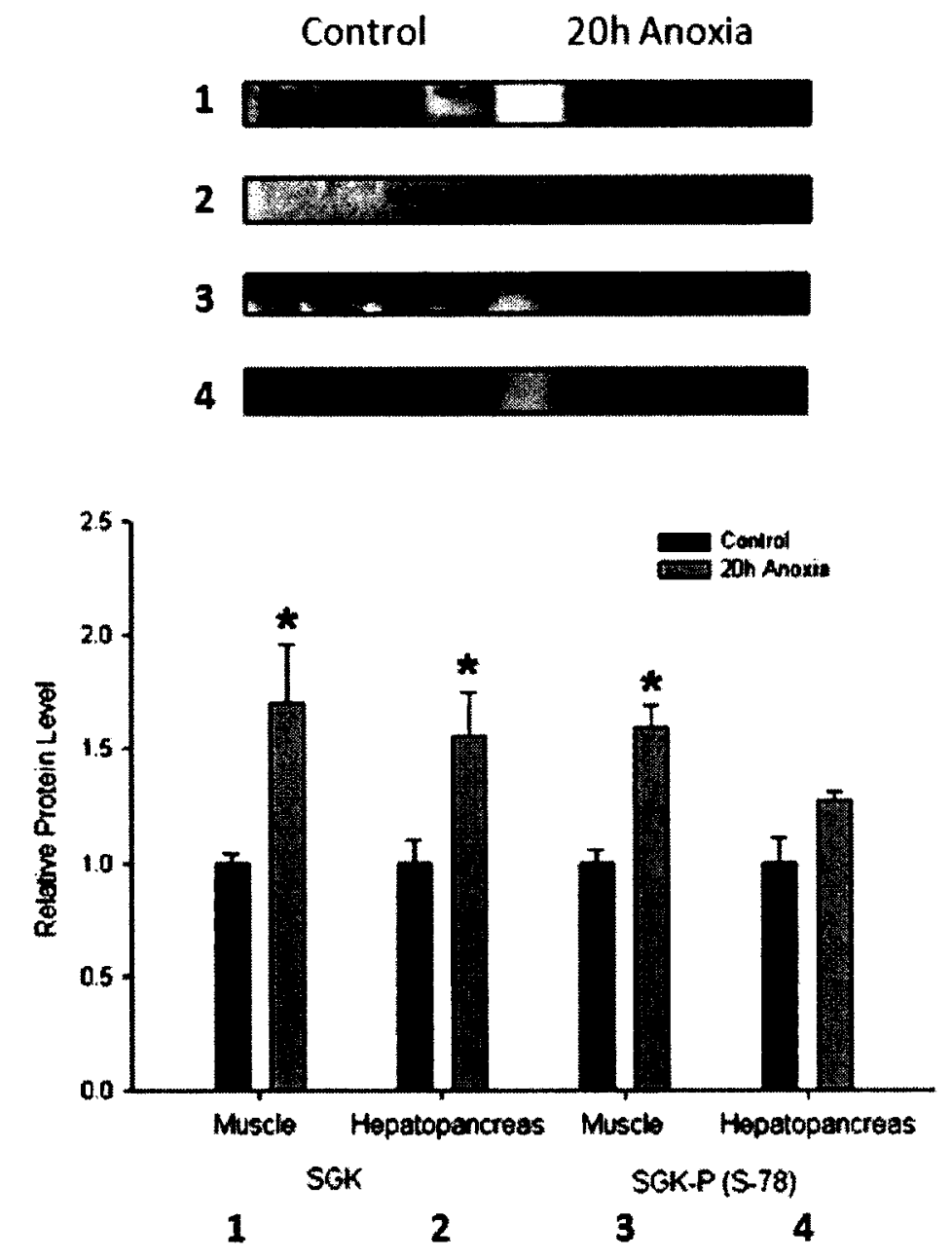

Figure 2.11: Effect of $20 \mathrm{~h}$ anoxia exposure on SGK protein levels and activation in $O$. virilis tissues.

Changes in SGK protein levels and phosphorylation of SGK at serine 78 in soluble protein extracts of crayfish tissues in response to anoxia, as determined by western immunoblotting.

Data are means $\pm S E M, n=4$ independent trials for muscle and hepatopancreas. * indicates significantly different from the corresponding control, $P<0.05$. 
Control

20h Anoxia

1

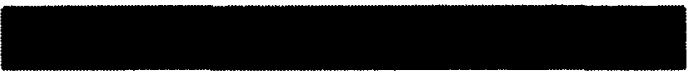

2

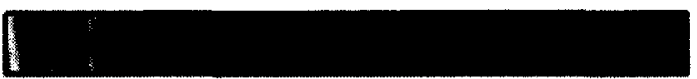

3

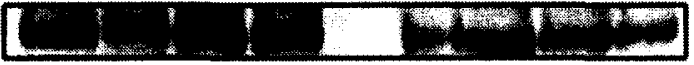

4
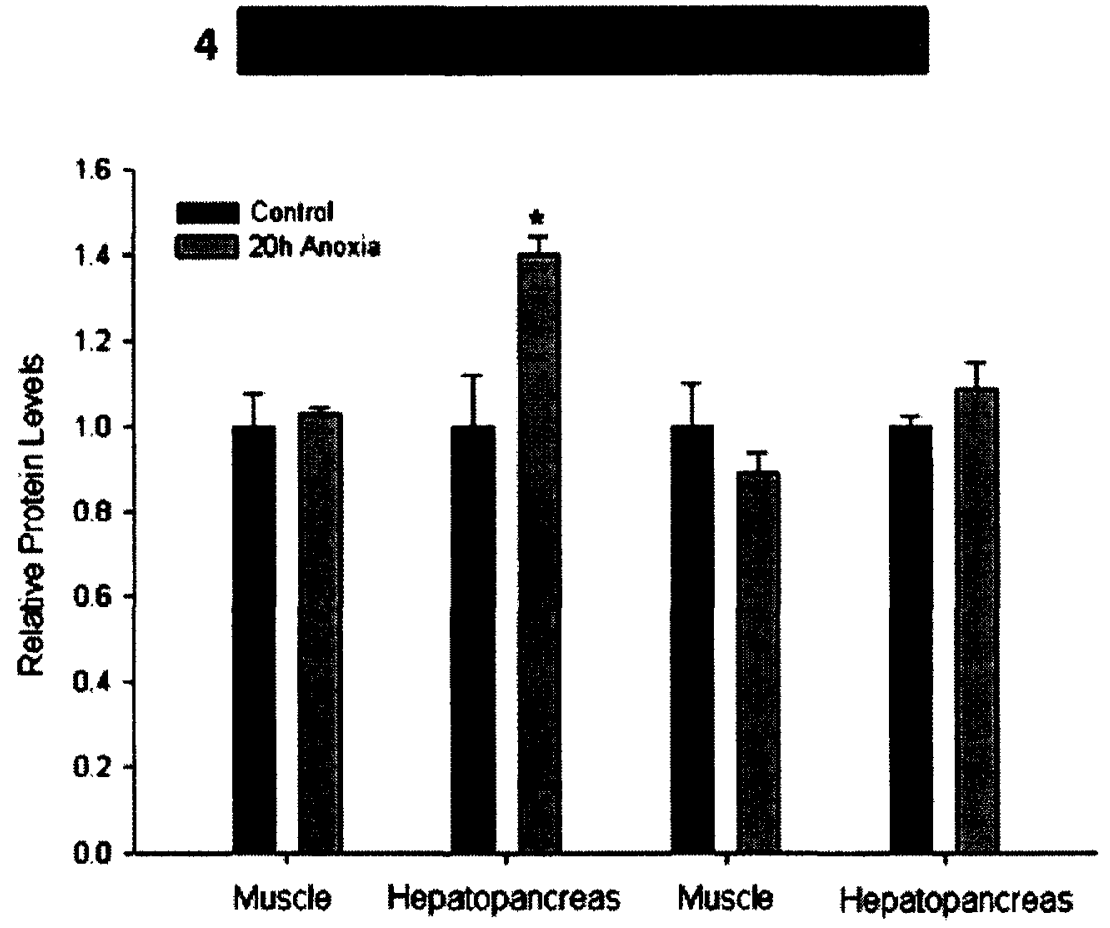

Foxo1

FoxO3

1

2

3

4

Figure 2.12: Effect of $20 \mathrm{~h}$ anoxia exposure on FoxO1 and FoxO3 protein levels in $O$. virilis tissues.

Changes in FoxO1 (left) and FoxO3 (right) protein levels in soluble protein extracts of crayfish tissues in response to anoxia, as determined by western immunoblotting. Data are means \pm SEM, $n=4$ independent trials for muscle and hepatopancreas. * indicates significantly different from the corresponding control, $P<0.05$. 


\section{Control 20h Anoxia}

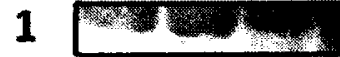

2

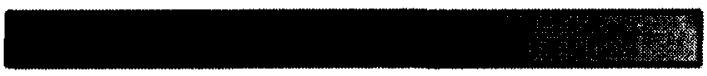

3

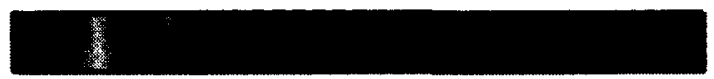

4
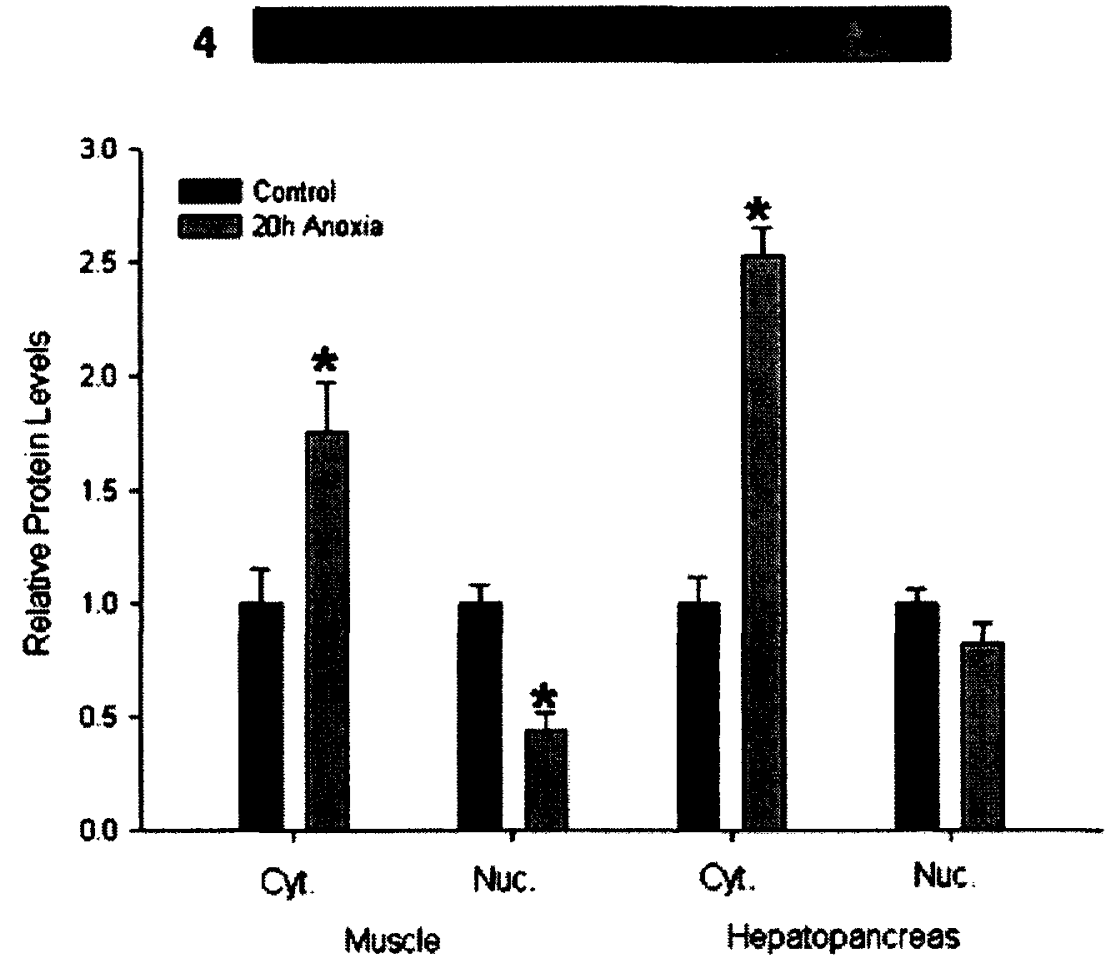

FoxO 1

1

2

3

4

Figure 2.13: Effect of $20 \mathrm{~h}$ anoxia exposure on the subcellular distribution of FoxO1 in $O$. virilis tissues.

Protein expression of FoxO1 in cytoplasmic and nuclear fractions of crayfish tissues (tail muscle, left; hepatopancreas, right) in response to anoxia, as determined by western immunoblotting.

Data are means $\pm S E M, n=4$ independent trials for muscle and hepatopancreas. * indicates significantly different from the corresponding control, $P<0.05$. 

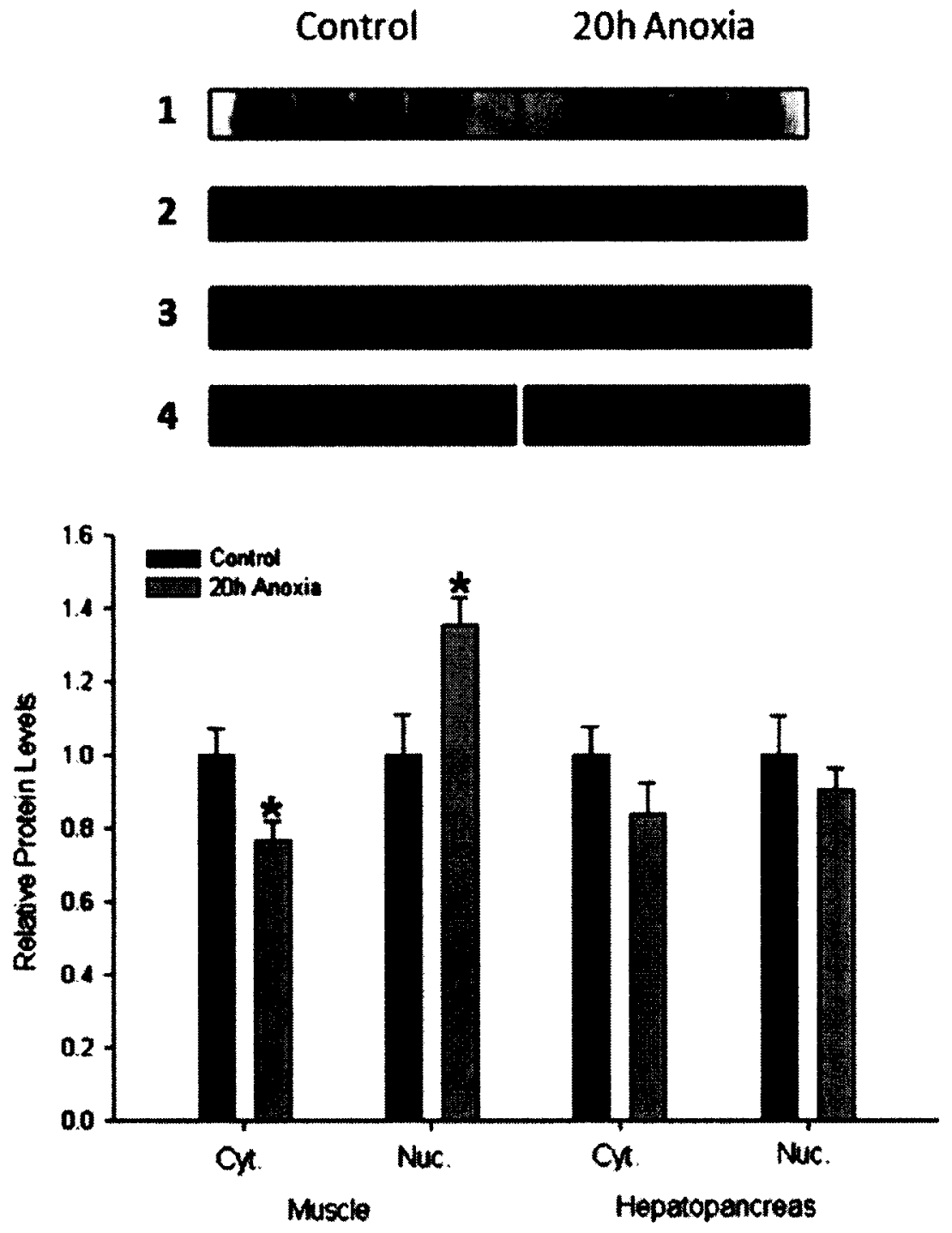

FoxO 3

1

2

3

4

Figure 2.14: Effect of $20 \mathrm{~h}$ anoxia exposure on the subcellular distribution of FoxO3 in $O$. virilis tissues.

Protein expression of FoxO3 in cytoplasmic and nuclear fractions of crayfish tissues (tail muscle, left; hepatopancreas, right) in response to anoxia, as determined by western immunoblotting.

Data are means $\pm S E M, n=4$ independent trials for muscle and hepatopancreas. * indicates significantly different from the corresponding control, $P<0.05$. 


\section{Control 20h Anoxia}

\section{1}

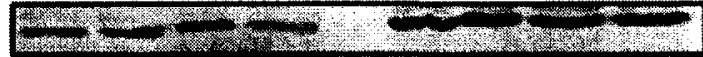

2

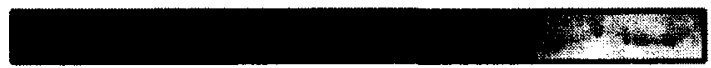

3

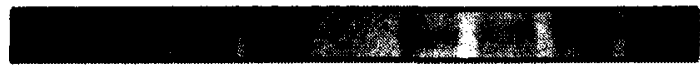

4
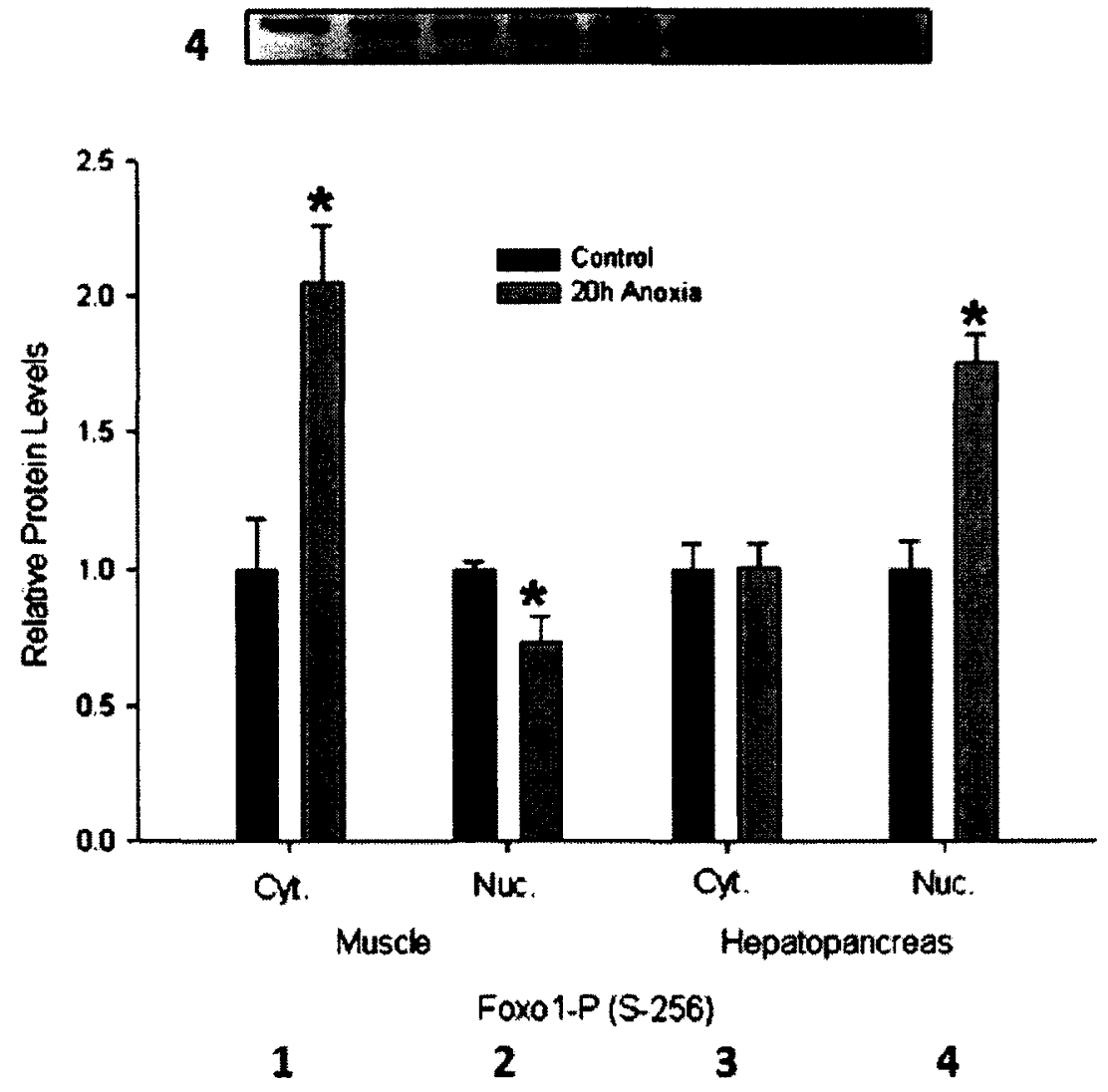

Figure 2.15: Effect of $20 \mathrm{~h}$ anoxia exposure on phosphorylation of FoxO1 in $O$. virilis tissues.

Phosphorylation of FoxOl, serine 256, in cytoplasmic and nuclear fractions of crayfish tissues (tail muscle, left; hepatopancreas, right) in response to anoxia, as determined by western immunoblotting.

Data are means \pm SEM, $n=4$ independent trials for muscle and hepatopancreas. * indicates significantly different from the corresponding control, $P<0.05$. 

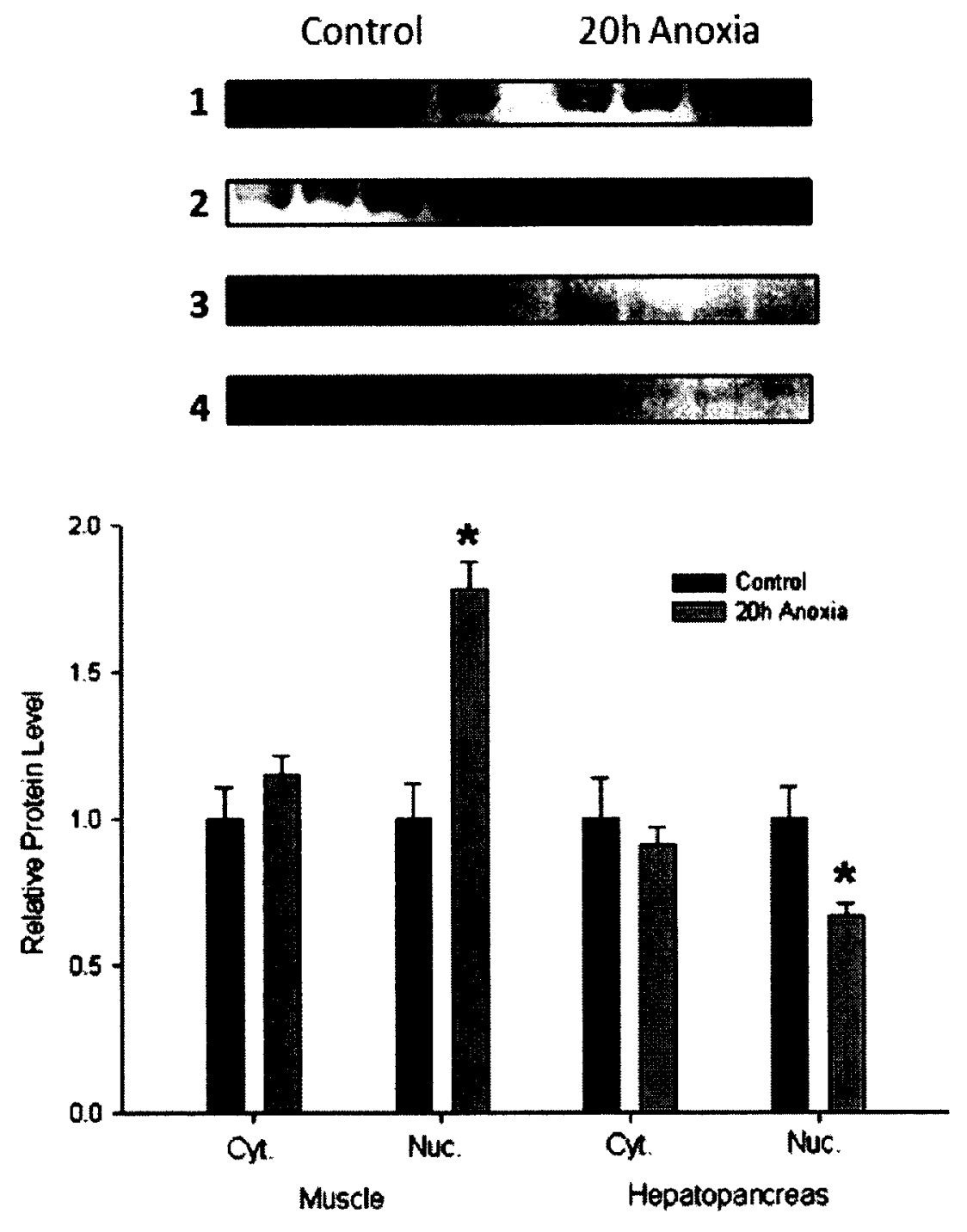

FoxO 3-P (S-253)

1

2

3

4

Figure 2.16: Effect of $20 \mathrm{~h}$ anoxia exposure on localization of phosphorylation of FoxO3 in 0 . virilis tissues.

Phosphorylation of FoxO3, serine 253, in cytoplasmic and nuclear fractions of crayfish tissues (tail muscle, left; hepatopancreas, right) in response to anoxia, as determined by western immunoblotting.

Data are means $\pm S E M, n=4$ independent trials for muscle and hepatopancreas. * indicates significantly different from the corresponding control, $P<0.05$. 

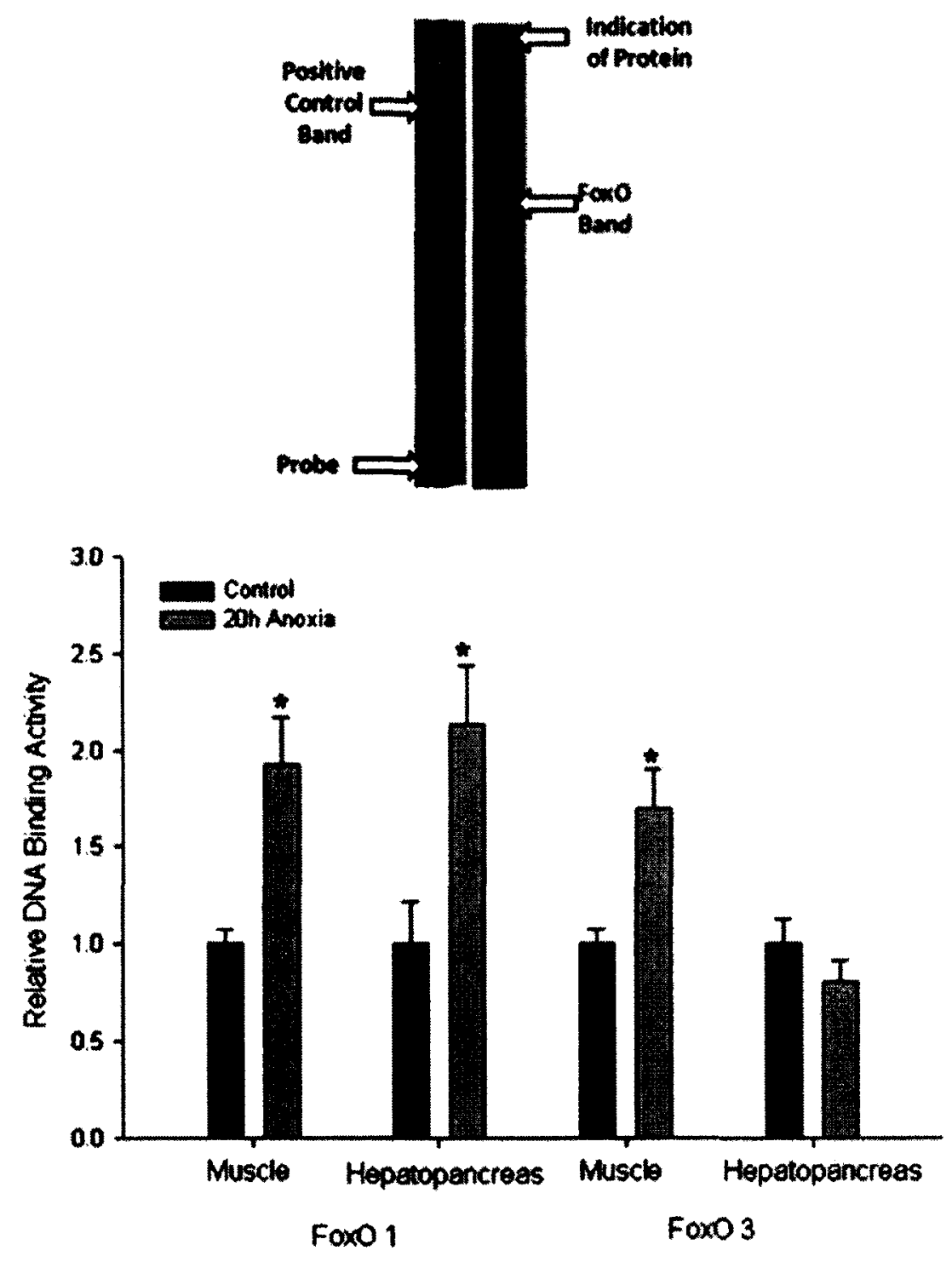

Figure 2.17: Effect of $20 \mathrm{~h}$ anoxia exposure on FoxO (1 and 3) DNA binding activity in $O$. virilis tissues.

Changes in the DNA-binding activity of FoxO1 (left) and FoxO3 (right) in nuclear extracts of muscle and hepatopancreas in response to anoxia, as determined by a transcription factor binding assay. Data are means \pm SEM, $n=4$ independent trials. * Indicates significantly different from the corresponding control, $P<0.05$.

Representative EMSA shown above the histogram. 


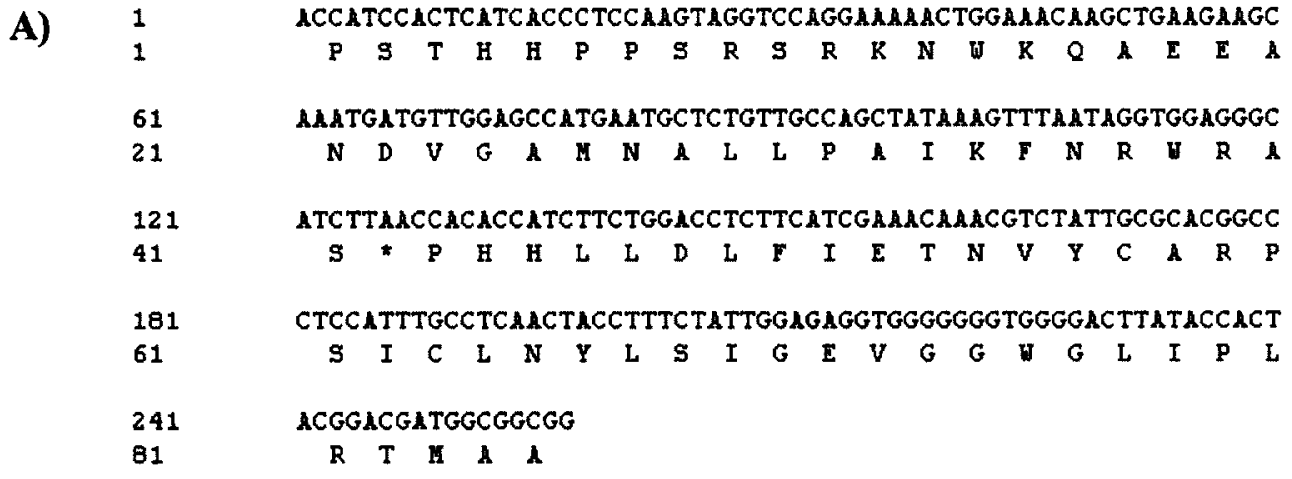

B) $\quad 100 \% 90 \% 800 \% 30 \% 500 \% 500 \% 400 \% 30 \%$

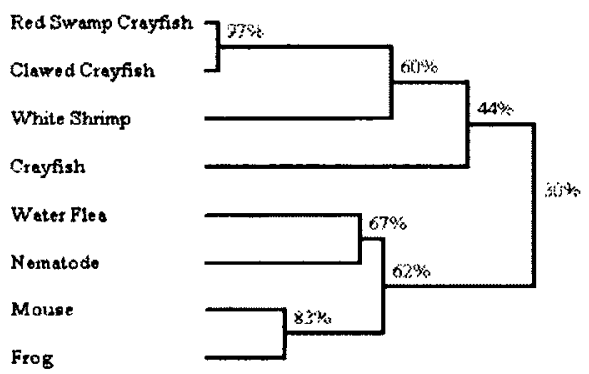

C)

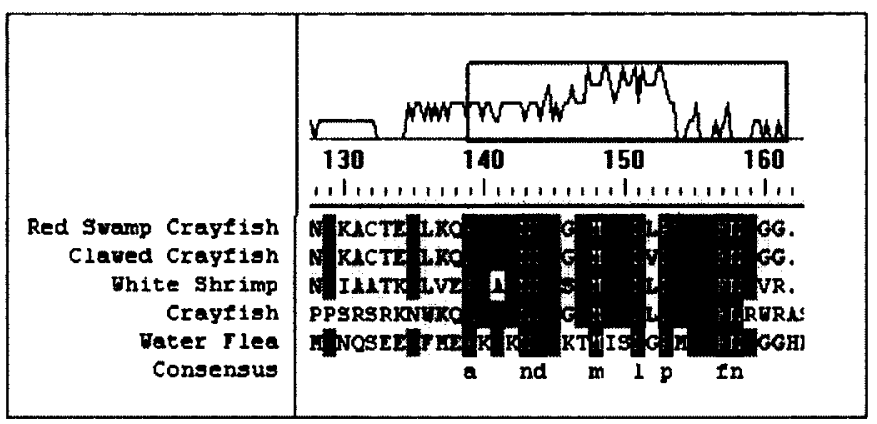

Figure 2.18: Partial nucleotide and deduced amino acid sequence of $O$. virilis MnSOD-like protein.

(A) The partial cDNA sequence of a MnSOD like protein, with the corresponding amino acid sequence is shown. Nucleotides and amino acids are numbered on the left. The nucleotide sequence was 198 nucleotides in length, and encoded 66 amino acids.

(B) Homology tree produced from an alignment of the partial $O$. virilis MnSOD like protein sequence with MnSOD (with Genbank accession numbers) from red swamp crayfish ( $P$. clarkia; ABX44762), clawed crayfish ( $P$. leptodactylus; ABI23722A), white crayfish (L.vannamei; AAP35680), water flea (D. pulex), nematode (C. elegans 
(NP_492290), mouse (M. musculus;AB34899) and African clawed frog $(X$. laevis;NP_001083968). There is currently no accession number for the sequenced $D$. pulex gene, but sequences may be found at http://wfleabase.org/.

(C) Amino acid sequence alignment of a portion of the crayfish MnSOD in comparison to invertebrate (arthropod) models (See section B for accession numbers).

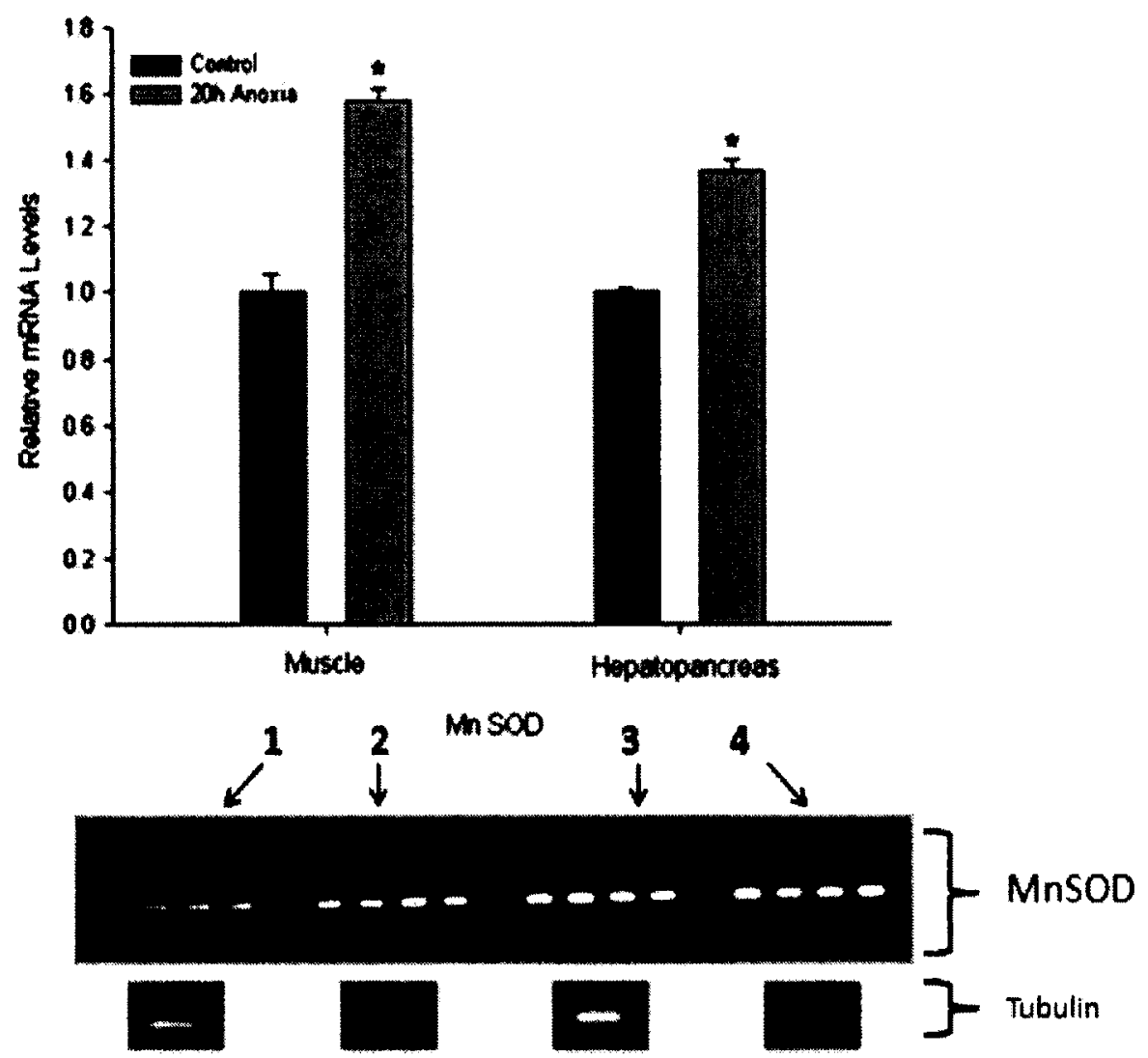

Figure 2.19: Effect of $20 \mathrm{~h}$ anoxic exposure on $\mathrm{MnSOD}$-like transcript expression in $O$. virilis tissues as determined by RT-PCR.

Histogram shows the normalized transcript levels under control and anoxic conditions; bands were normalized against the corresponding $\alpha$-tubulin bands (representative from each sample shown) amplified from the same sample. Data are means \pm SEM, $n=4$ independent trials for $\mathrm{mRNA}$ isolated from different crayfish. ${ }^{*}$ Indicates significantly different from the corresponding control, $P<0.05$.

$1=$ control muscle, $2=20 \mathrm{~h}$ anoxic muscle, $3=$ control hepatopancreas, $4=20 \mathrm{~h}$ anoxic hepatopancreas

Representative $M n S O D$ amplicons shown below 

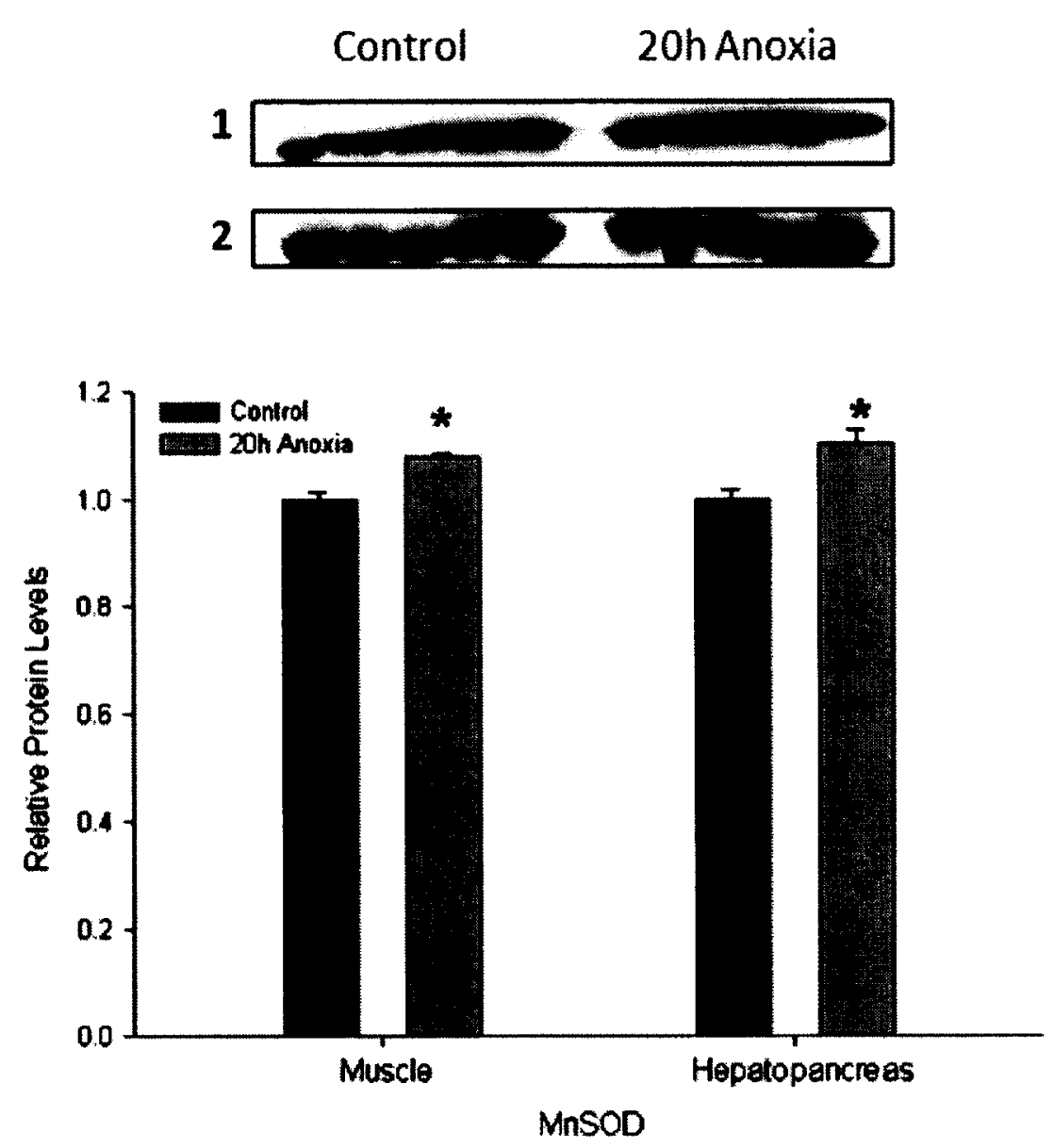

1

2

Figure 2.20: Effect of $20 \mathrm{~h}$ anoxia exposure on MnSOD expression in $O$. virilis tissues.

Changes in protein levels of MnSOD in total soluble protein fractions of crayfish tissues (tail muscle, left; hepatopancreas, right) in response to anoxia, as determined by western immunoblotting.

Data are means $\pm S E M, n=5$ independent trials for muscle and hepatopancreas. * indicates significantly different from the corresponding control, $P<0.05$. 

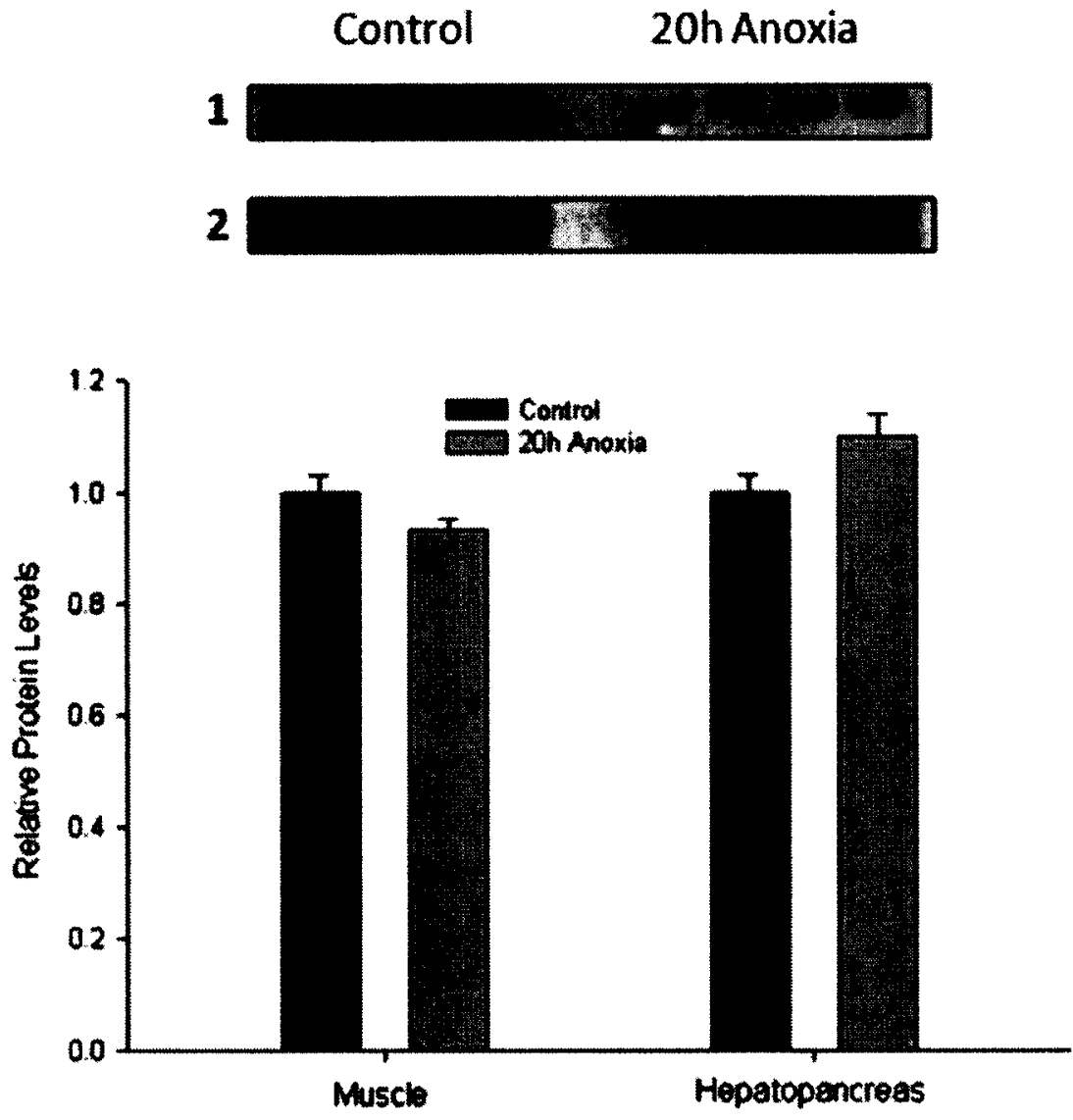

Catalase

1

Figure 2.21: Effect of $20 \mathrm{~h}$ anoxia exposure on catalase expression in $O$. virilis tissues.

Changes in protein levels of catalase in total soluble protein fractions of crayfish tissues (tail muscle, left; hepatopancreas, right) in response to anoxia, as determined by western immunoblotting.

Data are means \pm SEM, $n=4$ independent trials for muscle and hepatopancreas. * indicates significantly different from the corresponding control, $P<0.05$. 

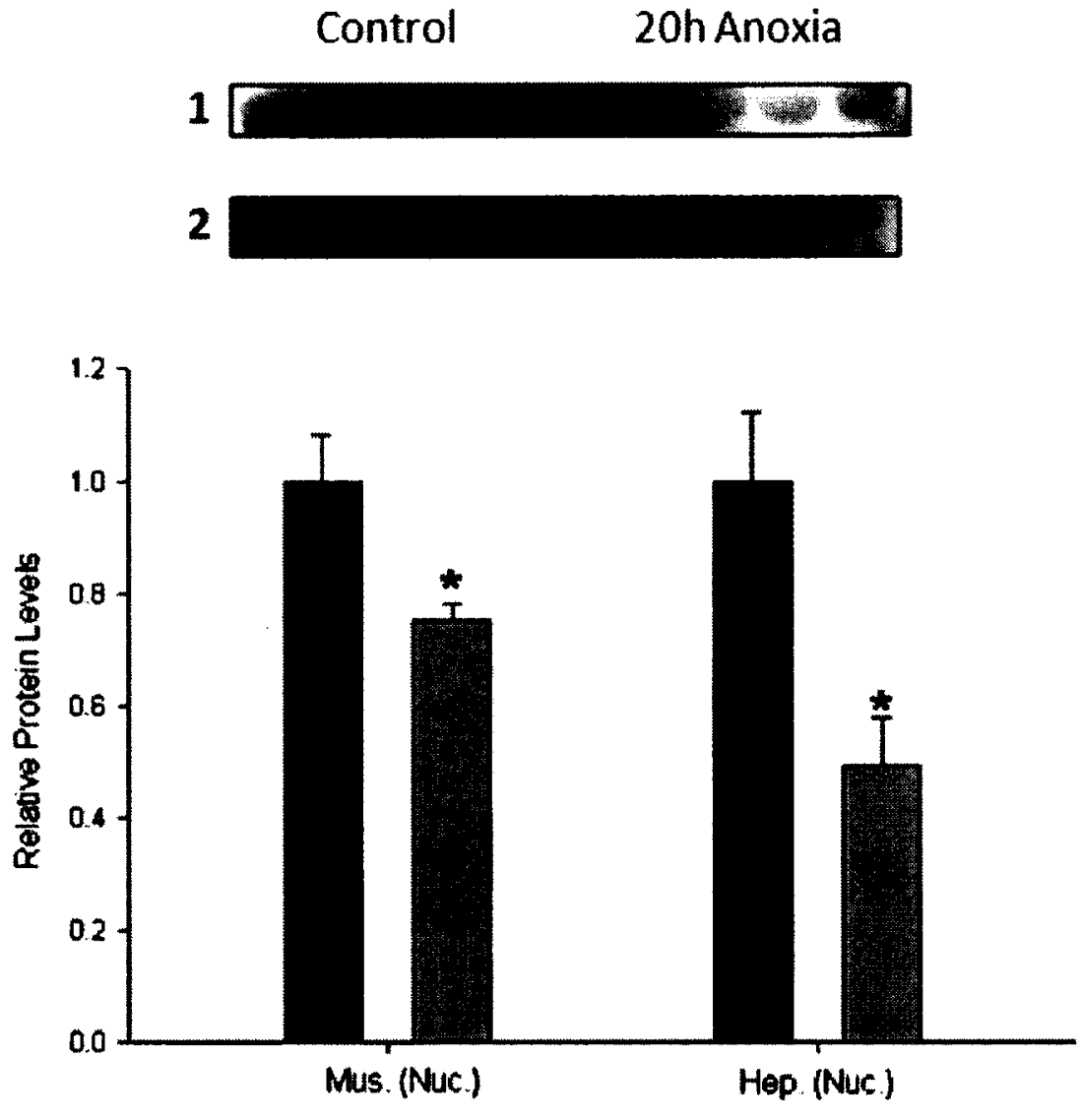

$14.3-3$

1

Figure 2.22: Effect of $20 \mathrm{~h}$ anoxia exposure on nuclear 14-3-3 expression in $O$. virilis tissues.

Changes in protein levels of 14-3-3 in nuclear protein fractions of crayfish tail muscle (left) and hepatopancreas (right) in response to anoxia, as determined by western immunoblotting.

Data are means \pm SEM, $n=4$ independent trials for muscle and hepatopancreas. * indicates significantly different from the corresponding control, $P<0.05$. 
Table 2.3 Chapter 2 data summary

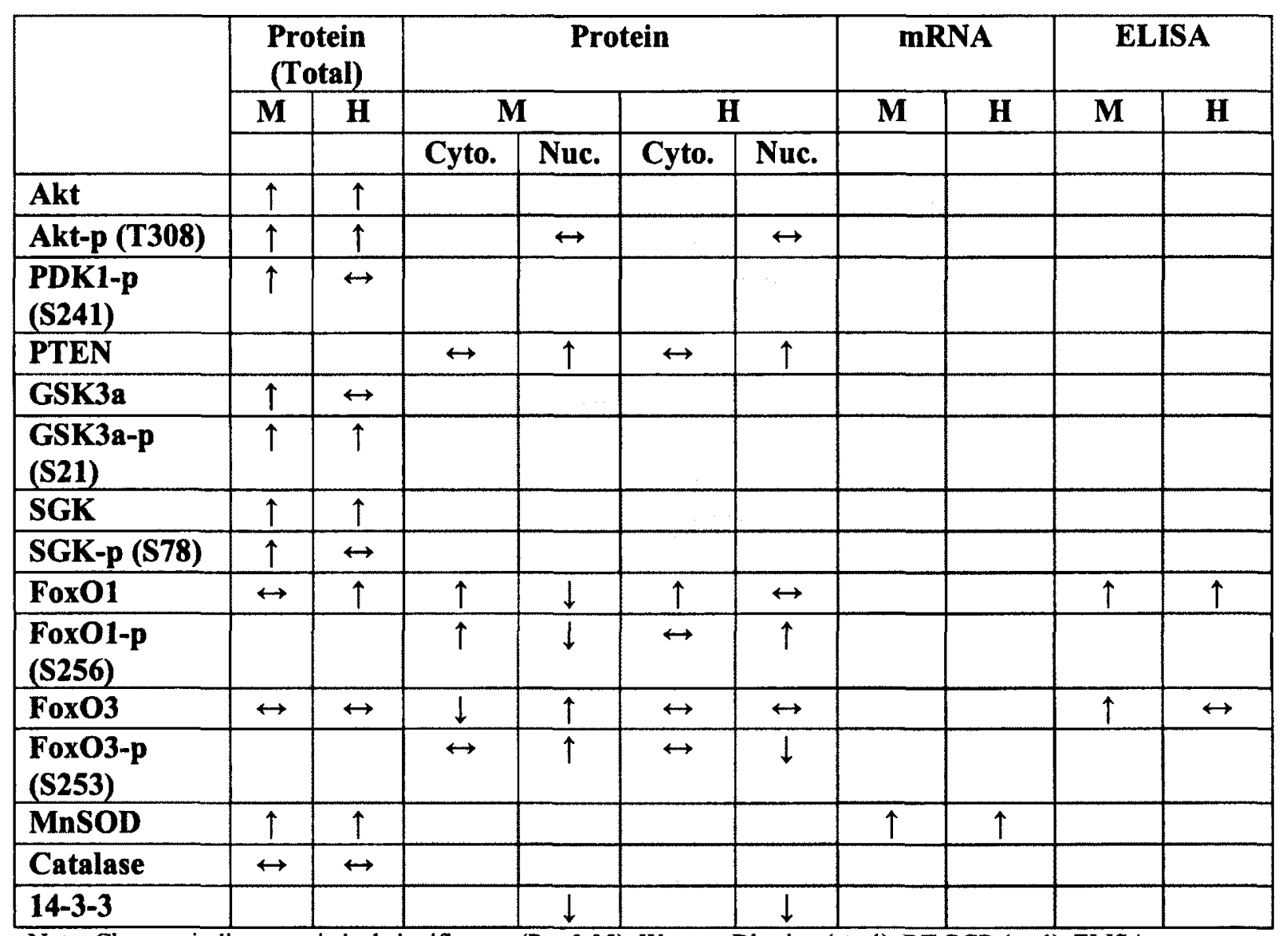

Note: Changes indicate statistical significance ( $P<0.05)$; Western Blotting $(n \geq 4)$, RT-PCR $(n \geq 4)$, ELISA $(n=4)$

Arrows indicate changes or level profiles, in response to anoxia 


\section{Chapter 3}

Smad and STAT signaling in freshwater crayfish; Regulation of developmental pathways under anoxia 


\subsection{Introduction}

The TGF- $\beta$ Signaling pathway:

Along with the Insulin/IGF pathway, in which Akt is the controlling element, the transforming growth factor $\beta$ (TGF- $\beta$ ) signaling pathway is one of the major paths regulating development in cells. Like the Insulin/IGF pathway, there are conflicting responses with it being involved in both proliferation and cell differentiation. The TGF- $\beta$ pathway mirrors the insulin/IGF pathway in that typically high activity in both pathways indicates conditions conducive to cellular growth, and indeed there are links between the two pathways, especially in the context of metabolic regulation (Narasimhan et al, 2011). The TGF- $\beta$ superfamily of signaling proteins includes a variety of growth and developmental members such as 'bone morphogenic proteins' (BMPs), activins and 'growth and differentiation factors' (GDFs), as well as the TGF- $\beta$ ligands themselves (Wu and Hill, 2009). The "growth" aspects of the pathway consume significant amounts of cellular energy, and as mentioned before, may be the target of repression under hypometabolic conditions. High levels of TGF- $\beta$ activity can also lead to tumorigenesis (Miyazono, 2011), and as such, suppression of TGF- $\beta$ activity may be indicative of antiproliferation or developmental responses within the cell. However, like the insulin/IGF pathway, the TGF- $\beta$ pathway has both proliferative and anti-proliferative responses.

\section{The Smad transcription factors:}

The primary downstream effectors of TGF- $\beta$ signaling are the 'mothers against decapentaplegic' transcription factors, the Smads (Wu and Hill, 2009). There are multiple members of the Smad family, and specificity in signaling is achieved by differential activity of the upstream TGF- $\beta$ receptors (type I and II) (FIG. 3.1) (Wrighton 
et al., 2009). These receptors are regulated by their phosphorylation state, which either results in pathway signal transduction, or their own degradation (Wrighton et al., 2009). If the two receptors are unable to interact due to physical inhibition or by a dephosphorylation event [ex. Type-I receptor dephosphorylation by protein phosphatase 2A (PP2A)], then TGF- $\beta$ signaling is inhibited, and the Smads are unable to function (Wrighton et al., 2009).

The Smad family of proteins are evolutionarily conserved transcription factors, and can be broken into three classes. The receptor ( $\mathrm{r}-$ ) Smads (Smads 1,2,3,5 and 8) are transcription factors that must be phosphorylated in order to enter the nucleus and carry out their functions. Once phosphorylated, the r-Smads will either complex with each other (Smad 2/3 and Smad1/5/8) or remain on their own (typically Smad 2), and require further binding to the 'complexing' Smad, Smad 4 (FIG. 3.1) (Wu and Hill, 2009). The final Smads, Smad 6 and 7, are inhibitory Smads (i-Smads), which inactivate Smad signaling (Wu and Hill, 2009). Phosphorylation is the key mechanism of both Smad activation and inactivation. For the r-Smads, double phosphorylation of the SXS motif (on the C-terminal), is necessary prior to complexing and transcription factor activity, however, phosphorylation of the 'linker' region may lead to inactivity (Wrighton et al., 2009). Once in the nucleus, the Smad complexes are able to bind a region in Smad responsive gene promoters that is highly conserved, and has been identified as 5'-GTCT3' (complement; 5'-AGAC-3') (Messagué et al., 2005). Nuclear expulsion, however, can occur through dephosphorylation (via protein phosphatase PPM1A), phosphorylation of the linker site and in the case of Smad 4, sumoylation (Wu and Hill, 2009). Degradation of the Smads is carried out by ubiquitin ligase mediated degradation (Smurf 1 and 2), and 
is signaled by phosphorylated residues (Wrighton et al., 2009). Among the kinases that inhibit Smad signaling by phosphorylation are cyclin dependent kinases (CDKs), GSK-3 (mentioned in the previous chapter) and members of the mitogen activated protein kinase (MAPK) family (specifically ERK and JNK) (Wrighton et al., 2009).

Along with phosphorylation control of the Smads, another significant contributing mechanism to their activity is co-factor binding of the Smads, both inside (TAZ, p300) and outside (Smad anchor for receptor activation; SARA) of the nucleus. In both cases these co-factors stabilize the Smad; the former preventing nuclear expulsion, and the latter promoting phosphorylation for nuclear translocation (Wrighton et al., 2009). The STAT family of transcription factors:

The signal transducer and activators of transcription' (STAT) family of proteins are, like the Smads, a family of highly conserved transcription factors involved mainly in pro-developmental signaling. The Stat transcription factors, in conjunction with their activating kinase from the Janus Kinase family (JAK), combine to form the JAK-STAT pathway which, like the TGF- $\beta$ pathway, is responsive primarily to growth factors and cytokines, and is mainly a developmental pathway (Wang and Levy, 2006). There are seven members in the STAT family, with Stat 1 and 3 being prominent members (Wang and Levy, 2006). Conserved activation of STAT transcription factors involves phosphorylation of tyrosine residues (ex. Tyrosine 701 of Stat 1), typically by JAK kinase, allowing movement into the nucleus for transcriptional activity (Wang and Levy, 2006).

Like the Smads, the STATs are heavily regulated at the point of nuclear translocation, with inhibitors often targeting STAT phosphorylation sites. The 
'suppressors of cytokine signaling' (SOCs) family of inhibitors prevent phosphorylation of STATs by binding the JAK receptor site, whereas members of the 'protein inhibitors of STATs' (PIAS) family, bind to phosphorylated STATs and block transcriptional activity (Stephanou and Latchman, 2003).

Mentioned earlier was the prominence of Stat 1 and 3 family members in pathway dynamics. In response to ischemia/reperfusion (I/R), a common occurrence in cases of stroke or heart attack where blood flow is severely restricted and then resumed, the two family members are functionally opposed (Stephanou, 2004). In response to this stress state, Stat 1 promotes apoptosis signaling, in part through the death receptor and ligand, Fas/Fas-L and protease caspase 1 (Stephanou, 2004). Stat 3, however, triggers protective responses through increased expression of anti-apoptotic proteins from the B-CellLymphoma (Bcl) family, Bcl-2 and Bcl-xl; proteins that are down regulated by Stat 1 (Stephanou and Latchman, 2003).

With regards to $C$. elegans dauer, and the response to adaptive metabolism, both Smads and STATs may be targeted given that pro-developmental roles are repressed during hypometabolism (see Appendix B.3).

In this chapter, the response of critical members of the Smad and STAT families are analyzed in response to anoxia stress in the crayfish. Protein and transcript levels along with DNA binding activity of selected family members are examined in order to analyze the involvement of these largely pro-developmental proteins in low oxygen survival. The studies also focus on the potential for interactions and parallel profiles of activity between the two pathways. What will become apparent is that despite the metabolic restrictions imposed on crayfish by prolonged exposure to anoxia, the response 
of these two pathways largely remains level in terms of maintaining protein and transcript levels. Both paths generally show maintained developmental responses under anoxia, with selected cases of altered activity.

\subsection{Materials and Methods}

\subsubsection{Animals}

Crayfish were treated and tissue samples were collected as described in Chapter 2.

\subsubsection{RNA extraction and $c D N A$ synthesis}

Total RNA was isolated from crayfish tissues and cDNA was reverse transcribed and serially diluted as described in Chapter 2.

\subsubsection{Primers}

Forward and reverse primers for Smad 3 and Stat 1 were designed from conserved regions in these genes based on DNA alignment of the sequences from several vertebrate and invertebrate species. Perfect (crayfish-specific) primers were subsequently designed for both transcripts and used for quantification after the crayfish sequences for these genes were obtained. Primers for the control gene, $\alpha$-tubulin, were as described in chapter 2. Primers and cDNA product sizes are listed in Table 3.1.

\subsubsection{RT-PCR}

PCR reactions were composed as described in Chapter 2. The PCR program was as follows: $7 \mathrm{~min}$ at $94^{\circ} \mathrm{C}$ followed by 35 cycles of $1 \mathrm{~min}$ at $94^{\circ} \mathrm{C}, 1 \mathrm{~min}$ at a predetermined annealing temperature $\left(53^{\circ} \mathrm{C}\right.$ for $S \operatorname{mad} 3,60^{\circ} \mathrm{C}$ for $\left.S t a t 1\right)$, and $1.5 \mathrm{~min}$ at $72^{\circ} \mathrm{C}$. The final step was $72^{\circ} \mathrm{C}$ for $10 \mathrm{~min}$. PCR products were separated on a $1 \%$ agarose gel stained with ethidium bromide, visualized using the ChemiGenius imaging system (Syngene, Frederick, MD, USA) under UV light and quantified using the GeneTools program. The bands from the most dilute cDNA sample that gave visible product were 
used for quantification to ensure that the products had not reached amplification saturation. PCR products were sequenced by DNA Landmarks (St.-Jean-sur-Richelieu, QC) and sequences were verified as encoding the correct genes using the program BLASTN (http://www.ncbi.nlm.nih.gov/blast) at the NIH.

\subsubsection{Protein extracts and western blotting}

Protein extracts were prepared from tissue samples of control and $20 \mathrm{~h}$ anoxic crayfish. Equal amounts of protein $(20-30 \mu \mathrm{g})$ were loaded into each lane of $10 \%$ SDS polyacrylamide gels, electrophoresed and then electroblotted onto polyvinylidene difluoride (PVDF) membranes as described in Chapter 2. After the transfer of proteins, membranes were blocked for $15 \mathrm{~min}$ in Tris buffered saline containing Tween-20 (TBST: $20 \mathrm{mM}$ Tris base, $140 \mathrm{mM} \mathrm{NaCl}, 0.1 \% \mathrm{v} / \mathrm{v}$ Tween-20) with $2.5 \%$ non-fat dried milk added. Blots were probed overnight with primary antibodies (listed in TABLE 3.2). All primary antibodies were diluted 1:1000 in TBST. After incubation, blots were washed several times with TBST and then incubated at RT for $1.5 \mathrm{~h}$ with HRP-linked goat antirabbit IgG (Cell Signaling, diluted 1:2000 in TBST). Immunoblots were developed using enhanced chemiluminescence reagents.

\subsubsection{Preparation of nuclear extracts and DNA-binding assay}

Cytoplasmic and nuclear extracts were prepared for DNA-binding assays as described in chapter 2. Aliquots containing equal amounts of protein from each sample ( $20 \mu \mathrm{g} /$ well) were then used to assess the amount of binding by Smad3 and Stat 1 to their respective DNA binding elements as described in Chapter 2. For Smad3, the sequence of the biotin-conjugated probe was 5'- Biotin-GAATGTATGTCTCAGATTAGCGAA-3', whereas the complementary sequence was 5'- TTCGCTAATCTGAGACATACATTC- 
3'. For Stat $1-p$, the sequence of the biotin-conjugated probe was 5'- Biotin-

CATGTTATGCATTCCCGTAAGTG-3', whereas the complementary sequence was 5'CACTTTACCGGGAATGCATAACATG- 3'.

Aliquots of nuclear extracts containing $20 \mu \mathrm{g}$ of protein were each combined with $50 \mu \mathrm{L}$ of $1 \times$ protein binding buffer [ $10 \mathrm{mM}$ Hepes, $\mathrm{pH} 7.9,50 \mathrm{mM} \mathrm{KCl}, 0.5 \mathrm{mM}$ EDTA, $3 \mathrm{mM} \mathrm{MgCl} 2,10 \% \mathrm{v} / \mathrm{v}$ glycerol, $0.5 \mathrm{mg} / \mathrm{mL}$ BSA, $0.05 \% \mathrm{NP}-40,1 \mu \mathrm{g}$ salmon sperm DNA (BioShop), $0.5 \mathrm{mM}$ DTT, and $\mathrm{NaCl}$ (a variable amount was used based on the volume of nuclear extract added, final concentration was always $40 \mathrm{mM}$ )]. The plate was incubated at RT with mild agitation for $75 \mathrm{~min}$ and then washed 4 times with wash buffer. Aliquots of $60 \mu \mathrm{L}$ of primary antibody for Smad3 and Statl-p (Cell Signaling), diluted 1:250 in PBS, were then added to the wells and the plate was incubated for $3 \mathrm{~h}$. The remainder of the procedure was as described in Chapter 2.

\subsubsection{Electromobility Shift Assay}

In order to confirm probe efficiency for the DNA-binding activity ELISA, an electromobility shift assay was performed to visualize probe binding. First, biotinylated probe from the DNA binding activity assay $(250 \mathrm{pmol} / \mu \mathrm{l})$ was diluted to $10 \mathrm{pmol} / \mu \mathrm{l}$. Aliquots of $4 \mu \mathrm{l}(8 \mu \mathrm{g})$ of nuclear extract were mixed with $1 \mu \mathrm{DEPC}$ treated $\mathrm{H}_{2} \mathrm{O}$ and 4 $\mu$ I EM SA master mix; 5X EMSA binding buffer contained [50mM Tris $\mathrm{HCl}, \mathrm{pH} 7.8$, $250 \mathrm{mM} \mathrm{NaCl}, 5 \mathrm{mM}$ EDTA and $25 \%$ glycerol combined with $1 \mu \mathrm{g} / \mu \mathrm{l}$ Poly d(I-C) in a 1:1 v:v ratio. Samples were vortexed and incubated at RT for $5 \mathrm{~min}$ and then $1 \mu \mathrm{lTF}$ probe was added and the mixture was vortexed again before placing in the thermocycler for $30 \mathrm{~min}$ at $15^{\circ} \mathrm{C}$. The positive control was biotinylated E2F Probe (Panomics EMSA Kit 7104) tested with turtle (Trachemys scripta elegans) liver nuclear extract, in the same 
mix as above. Negative controls for no protein and no probe were also run, with adjustments to water volume as needed to keep total volume at $9 \mu \mathrm{l}$.

Following incubation, samples were mixed with $1 \mu 16 x$ DNA Loading dye (BioShop) prior to loading on a $6 \%$ non-denaturing polyacrylamide gel [ $1 \mathrm{ml} 10 \mathrm{X} \mathrm{TBE}$ (chilled), $4 \mathrm{ml} 30 \%$ bis-acrylamide, $625 \mu 180 \%$ glycerol, $14.375 \mathrm{ml}$ deionized sterile $\mathrm{H}_{2} \mathrm{O}$, $300 \mu 110 \%$ APS and $20 \mu 1$ TEMED - total $2 \times 10 \mathrm{ml} /$ gel). The gels were cast as in the Western blotting procedure (above). Gels were pre- run in chilled $0.5 \times \mathrm{TBE}$ for $10 \mathrm{~min}$ at $120 \mathrm{~V}$. Samples were then loaded into the gels and run at $120 \mathrm{~V}$ for $70 \mathrm{~min}$ at $4^{\circ} \mathrm{C}$ (fridge). Following electrophoresis, proteins were transferred to Pall Biodyne B nylon membranes. Membranes were pre-soaked in $0.5 \mathrm{x}$ TBE and then gels were sandwiched to membranes as described for the Western blotting protocol. Proteins were transferred in $0.5 x$ TBE (chilled) for $30 \mathrm{~min}$ at $300 \mathrm{~mA}$ at $4^{\circ} \mathrm{C}$. Once transfer was complete, membranes were stored between dry filter paper, and the oligos were fixed to membranes in an oven $\left(80^{\circ} \mathrm{C}\right)$ for $1 \mathrm{~h}$. Following this, membranes were washed on a rocking platform with $5 \mathrm{ml}$ (per membrane) of 1x EMSA Blocking Buffer (diluted from Panomics CS7535) for 30 min at RT. The blocking buffer was decanted and to it $5 \mu \mathrm{l} / \mathrm{ml}$ Streptavidin-HRP mixture (BioShop) was added. This mixture was reapplied to the membrane and left to incubate on a rocking platform for a further $30 \mathrm{~min}$ at RT. Finally, membranes were washed 3 times, consecutively, with $1 \mathrm{x}$ wash buffer (diluted from Panomics 7537 ). The membranes were then exposed using ECL solutions as in the Western blotting protocol.

\subsubsection{Data Analysis}

All data were analyzed as described in Chapter 2. 
Table 3.1 List of Primers used in Chapter 3

\begin{tabular}{|c|c|c|c|}
\hline Gene/primer & Primer sequence (5'-3') & $\begin{array}{l}\text { Annealing } \\
\text { temp. }\left({ }^{\circ} \mathrm{C}\right)\end{array}$ & $\begin{array}{l}\text { Expected } \\
\text { product size } \\
\text { (bp) }\end{array}$ \\
\hline $\begin{array}{l}\text { Smad } 3 \text { perfect } \\
\text { forward }\end{array}$ & TCCACCTACTCTGAGAGTCT & \multirow[b]{2}{*}{53} & \multirow[b]{2}{*}{$\sim 160$} \\
\hline $\begin{array}{l}\text { Smad } 3 \text { perfect } \\
\text { reverse }\end{array}$ & CTGTCGTCTGTACTCCAGAT & & \\
\hline $\begin{array}{l}\text { Stat } 1 \text { perfect } \\
\text { forward }\end{array}$ & AACGTTGAAGAGGCGCAGGT & \multirow[b]{2}{*}{60} & \multirow[b]{2}{*}{$\sim 360$} \\
\hline Stat 1 perfect reverse & TAGAGTCGAGGTTGCTATGG & & \\
\hline
\end{tabular}

Table 3.2 List of primary antibodies used in Chapter 3

\begin{tabular}{|l|l|l|}
\hline \multicolumn{1}{|c|}{$\begin{array}{c}\text { Primary } \\
\text { Antibody }\end{array}$} & \multicolumn{1}{|c|}{ Company } & Catalogue \# \\
\hline $\begin{array}{l}\text { Smad } 1 / 5-p \\
(S 463 / 465)\end{array}$ & Cell Signaling (Danvers, MA, USA) & $\# 9516$ \\
\hline $\begin{array}{l}\text { Smad } 2-p \\
(\text { S465/467) }\end{array}$ & Cell Signaling (Danvers, MA, USA) & $\# 3108$ \\
\hline Smad 3 & Cell Signaling (Danvers, MA, USA) & $\# 9523$ \\
\hline Smad 4 & Cell Signaling (Danvers, MA, USA) & $\# 9515$ \\
\hline STAT1-p (T701) & Cell Signaling (Danvers, MA, USA) & $\# 9171$ \\
\hline STAT2-p (T690) & Cell Signaling (Danvers, MA, USA) & $\# 4441$ \\
\hline STAT3-p (T705) & Cell Signaling (Danvers, MA, USA) & $\# 9145$ \\
\hline STAT5 & Cell Signaling (Danvers, MA, USA) & $\# 9363$ \\
\hline STAT6 & Cell Signaling (Danvers, MA, USA) & $\# 9362$ \\
\hline
\end{tabular}




\subsection{Results}

3.3.1. Protein expression of Smad 1/5-p in response to anoxia

When Smads are phosphorylated they can form active complexes that migrate to the nucleus to stimulate transcription of genes under their control. Hence, changes in the relative phosphorylation state of Smads in response to stress (anoxia in this case) gives a good measure of the relative state of transcription of Smad-regulated genes; i.e. a significant increase in phosphorylation of Smad proteins at specific sites is indicative of enhanced Smad-mediate transcriptional activity. The polyclonal anti-Smad 1/5-p antibodies detect phosphorylation of the proteins at serine $463 / 465$. The antibodies crossreacted with a protein band of $\sim 60 \mathrm{kDa}$ in crayfish extracts, which is the expected size of Smad 1 and Smad 5. Changes in relative phosphorylation levels of Smad1/5-p (Ser $463 / 465$ ) in response to $20 \mathrm{~h}$ anoxia were assessed using immunoblotting in extracts from both tail muscle and hepatopancreas (FIG. 3.3). Relative phosphorylation of Smad1/5 was unchanged in tail muscle from anoxic crayfish compared with aerobic controls. However, in hepatopancreas a strong increase in phosphorylation of Smad 1 and 5 was seen after $20 \mathrm{~h}$ anoxia, with Smad1/5-p content rising by $2.8 \pm 0.1$ fold $(\mathrm{P}<0.05)$.

\subsubsection{Protein expression of Smad 2-p in response to anoxia}

Changes in the relative phosphorylation levels of Smad2-p (phosphorylated at serine 465/467) in response to anoxia were also assessed in crayfish tail muscle and hepatopancreas using immunoblotting (FIG.3.4). The polyclonal anti-Smad 2-p antibodies cross-reacted with a protein band of $\sim 60 \mathrm{kDa}$, which is its expected size. Again, the phosphorylation state was unaffected by anoxia in tail muscle, but a strong significant increase occurred in hepatopancreas, with Smad-2 phosphorylation increasing 
by $2.4 \pm 0.1$ fold $(\mathrm{P}<0.05)$ after $20 \mathrm{~h}$ of anoxia exposure.

\subsubsection{Nuclear expression of Smad 2-p in crayfish tissues}

In order to determine whether Smad 2-p translocated to the nucleus in response to anoxia, western immunoblotting was performed on nuclear and cytoplasmic extracts from crayfish tissues (FIG.3.5). While there was no increase in the relative amount of Smad 2 phosphorylation detected in nuclear extracts of either tissue, there were significant fluctuations in cytoplasmic phosphorylation levels. In tail muscle, the relative amount of Smad 2 phosphorylation detected in cytoplasmic extracts decreased during anoxia by 35 $\pm 4 \%$, whereas in hepatopancreas cytoplasmic phosphorylation of Smad 2 increased by $1.15 \pm 0.02$ fold during anoxia as compared with control values (both $\mathrm{P}>0.05$ ).

\subsection{4. cDNA cloning of crayfish Smad 3}

A PCR product of $166 \mathrm{bp}$ was retrieved from total RNA prepared from crayfish tail muscle and hepatopancreas using Smad 3 primers that were designed from the Smad 3 consensus sequence from several vertebrate and invertebrate species. The product was analyzed by BLAST, and did not appear to conform to other Smad sequences. The nonspecific identity of the product is in keeping with low homologies for other crustacean gene products (there is very limited amounts of data about crustacean sequences in gene banks). As such, the crayfish sequence will be identified as a Smad 3-like protein.

FIG.3.6A shows the partial nucleotide sequence with the corresponding amino acid sequence that encoded 52 amino acids. FIG.3.6B shows the homology between the isolated cDNA from $O$. virilis and the comparable fragment from the water flea.

FIG.3.6C shows a homology tree produced from the alignment of the partial $O$. virilis Smad3-like cDNA sequence with Smad from fruit fly (Drosophila melanogaster) and 
water flea (Daphnia pulex). The homology tree shows the separation of sequence homology. The water flea and fruit fly exhibit the closest similarity with $61 \%$ homology. The crayfish holds $40 \%$ similarity to these arthropods.

\subsubsection{Transcript expression of Smad 3 in response to anoxia}

Changes in the relative transcript levels of Smad 3 in response to $20 \mathrm{~h}$ of anoxia were assessed using RT-PCR and redesigned perfect primers from the crayfish sequence (FIG.3.7). Representative RT-PCR bands of Smad 3 amplicons are shown in conjunction with a graph showing the mean changes in the relative levels of these transcripts in response to anoxia. Smad 3 transcript levels were normalized against tubulin bands that were amplified from the same tissue samples. Smad 3 transcript levels remained constant in the hepatopancreas throughout anoxia, whereas in tail muscle anoxia triggered a significant $(1.8 \pm 0.1$ fold $)$ increase $(\mathrm{P}<0.05)$ in transcript levels.

\subsubsection{Protein expression of Smad 3 in response to anoxia}

Following analysis of Smad 3 transcript levels, changes in the levels of Smad 3 protein were also assessed using immunoblotting (FIG.3.8). The polyclonal anti-Smad 3 antibodies cross-reacted with a protein band of $\sim 52 \mathrm{kDa}$, which is the expected size. Relative protein levels remained constant over the anoxia exposure in the hepatopancreas as compared with controls, whereas (in accordance with the transcript level analysis) protein levels increased in the tail muscle by $1.4 \pm 0.08$ fold $(P<0.05)$.

\subsubsection{DNA binding activity of Smad 3 in response to anoxia}

Changes in the DNA-binding activity of nuclear Smad 3 in response to anoxia were assessed using a transcription factor binding assay in extracts of tail muscle and hepatopancreas (FIG.3.9). The relative amount of DNA binding showed no change in tail 
muscle nuclear extracts, despite the increases in protein and transcript noted above, but did increase in hepatopancreas nuclear extracts. DNA-binding activity by Smad 3 increased by $1.6 \pm 0.2$ fold, in response to 20 hour anoxic exposure $(P<0.05)$. Probe specificity is confirmed through EMSA banding.

\subsubsection{Protein expression of Smad 4 in response to anoxia}

Changes in the protein levels of Smad 4 in response to anoxia were also assessed using immunoblotting (FIG.3.10). The polyclonal anti-Smad 4 antibodies cross-reacted with a protein band of $\sim 70 \mathrm{kDa}$, which is its expected size. Protein levels, relative to the control condition remained stable over the anoxia exposure in both tail muscle and hepatopancreas.

\subsection{9. cDNA cloning of crayfish Stat 1}

A PCR product of $361 \mathrm{bp}$ was retrieved using the perfect Stat 1 primers (designed from the consensus sequence from several vertebrate and invertebrate species) from total RNA prepared from crayfish tail muscle and hepatopancreas. The product was analyzed by BLAST, and did not appear to conform to other Stat sequences. The non-specific identity of the product is in keeping with low homologies for other crustacean gene products, and as such the gene product will be identified as a Stat 1-like protein. FIG.3.11A shows the partial nucleotide sequence that encoded 110 amino acids. FIG.3.11B shows the homology between the isolated cDNA from $O$. virilis and the comparable fragment from the brine shrimp. FIG.3.11C shows a homology tree produced from the alignment of the partial $O$. virilis STAT1-like cDNA sequence with STAT1 from kuruma prawn (Marsupenaeus japonicus) and the brine shrimp (Artemia franciscana). The homology tree shows the separation of sequence homology, within the invertebrate 
arthropods. There is $55 \%$ similarity between the crayfish and the Kuruma prawn, while the brine shrimp holds $42 \%$ similarity to both.

\subsubsection{Transcript expression of Stat 1 in response to anoxia}

Changes in the relative transcript levels of Stat 1 in response to $20 \mathrm{~h}$ of anoxia were assessed using RT-PCR and perfect primers designed from the crayfish sequence (FIG.3.12). Representative RT-PCR bands of Stat 1 amplicons are shown along with a histogram showing the mean normalized levels (tubulin bands amplified from the same samples were used for normalization). Stat $I$ transcript levels remained constant in hepatopancreas during anoxia exposure, whereas levels increased significantly (by $1.4 \pm$ 0.02 fold $)(P<0.05)$ in tail muscle.

\subsubsection{Total and nuclear protein expression of Stat 1-p in response to anoxia}

Changes in the relative amounts of Stat 1 (tyrosine 701) phosphorylation in response to anoxia were assessed using immunoblotting (FIG.3.13). The polyclonal antiStat 1-p antibodies cross-reacted with a protein band at $\sim 91 \mathrm{kDa}$, the expected size. Changes in total phosphorylation levels are shown for tail muscle and hepatopancreas as well as levels in the nuclear fraction from each tissue. Total Stat 1 phosphorylation levels increased significantly during anoxia. In tail muscle the relative levels of Stat 1-p (Tyr 701) increased by $1.4 \pm 0.1$ fold, conforming with transcript level analysis, whereas phosphorylation in the hepatopancreas increased by $1.2 \pm 0.06$ fold (both $\mathrm{P}<0.05$ ). In contrast, the relative amount of Stat 1-p (Tyr 701) in nuclear fractions decreased significant in both tissues under anoxia, relative to aerobic control. In tail muscle, phosphorylation decreased by $15 \pm 3 \%$ and in hepatopancreas the drop was $25 \pm 5 \%$ (P $<0.05)$. 


\subsubsection{DNA binding activity of Stat 1 in response to anoxia}

Changes in the DNA-binding activity of nuclear (phospho) Stat 1 in response to anoxia were assessed using a transcription factor binding assay (FIG.3.14). DNA binding in tail muscle extracts suggested a decrease (not statistically significant), which accords with nuclear profiling. In hepatopancreas, a strong significant decrease in DNA binding activity by Stat 1 occurred, a decrease of $82 \pm 18 \%(P<0.05)$ in response to $20 \mathrm{~h}$ anoxic exposure. Probe specificity is confirmed through EMSA banding.

\subsubsection{Protein expression of Stat 2-p in response to anoxia}

Changes in the phosphorylation levels of Stat 2 (phosphorylated at tyrosine 690) in response to anoxia were assessed using immunoblotting (FIG.3.15). The polyclonal anti-Stat 2-p antibodies cross-reacted with a protein band of $\sim 110 \mathrm{kDa}$, which is its expected size. The relative phosphorylation levels of Stat 2-p (Try 690) were unchanged in tail muscle during anoxia. In the hepatopancreas, however, a significant decrease in phosphorylation occurred, dropping by $40 \pm 10 \%(\mathrm{P}<0.05)$ during anoxia.

\subsubsection{Protein expression of Stat 3-p in response to anoxia}

Changes in the phosphorylation of Stat 3 (phosphorylated at tyrosine 705) in response to anoxia are shown in FIG.3.16. The polyclonal anti-Stat 3-p antibodies crossreacted with a protein band of $\sim 85 \mathrm{kDa}$, the expected size. Phosphorylation levels in tail muscle showed small but significant increases compared with aerobic controls, rising by $1.2 \pm 0.03$ fold over anoxia $(P<0.05)$. However, no significant change occurred in hepatopancreas during anoxia.

\subsubsection{Protein expression of Stat 5 and 6 in response to anoxia}

Protein levels of Stat 5 and Stat 6 are shown in FIG.3.17. The polyclonal anti-Stat 
5 and anti-Stat 6 antibodies cross-reacted with protein bands of $\sim 90 \mathrm{kDa}$ and $\sim 100 \mathrm{kDa}$, respectively, which are their expected sizes. Neither Stat 5 nor Stat 6 protein levels changed significantly in either muscle or hepatopancreas between control and anoxic conditions.

\subsection{Discussion}

The TGF- $\beta$ pathway, along with the JAK-STAT pathway, have long been recognized as critical pathways involved in development and differentiation (Raftery and Sutherland, 1999; Padgett et al., 1998; Watanabe and Arai, 1996; Dearolf, 1999). However, as roles for these pathways are increasingly documented in disease states, notably cancer (Inman, 2011; Wang and Huang, 2010), it has become important to also evaluate their involvement in biochemical adaptation to environmental stress. Studying the responses of Smads and STATs in a model of anoxia resistance is particularly relevant since tumors often survive in a low oxygen (hypoxia) situation and, hence, it is important to know how these transcription factors respond naturally to low oxygen in anoxia resistant species.

\section{Smad regulation in response to anoxia resistance}

Changes to Smad family members under anoxia, indicate generally increased Smad activity in crayfish tissues. Unlike the hypometabolic dauer state (see Appendix A) in the nematode, C. elegans (Daniels et al., 2000), there does not appear to be a suppression of TGF- $\beta$ signaling through the Smad transcription factors in response to anoxia-induced hypometabolism in the crayfish (Table 3.3). In fact, amongst the proteins measured, including phospho-forms (Smad 1/5-p, Smad 2-p) and total protein levels (Smad 3 and 4), there were no instances of suppression of protein expression, suggesting 
that at very least, the pro-development signals mediated by the Smads are still as functional in anoxia as they were in normoxia. The phospho sites targeted on the Smads measured (Ser 463/465 on Smad 1/5-p and Ser 456/467 on Smad 2-p), are both in the SSXS motif of the C-terminus, and it is known that phosphorylation at these sites is needed to permit Smad complexing and nuclear translocation to trigger gene expression. Hence, the phospho-specific antibodies measure active Smads and the increase in relative phosphorylation at these sites on both Smad 1/5 and Smad 2 in the hepatopancreas under anoxia indicates an activation of these Smads. However looking at the subcellular distribution of phospho-Smad 2 (Ser 456/467), we can see that protein level changes in both muscle and hepatopancreas occur in the cytoplasm only and the relative levels of phospho-Smad 2 in the nucleus remain constant between control and anoxic conditions. Being the only Smad that is capable of entering the nucleus without homo-dimerization with another r-Smad, the presence of Smad-2 in the nucleus is often a successful indicator of TGF- $\beta$ signal transduction (Wu and Hill, 2009). Hence, despite an increase in the overall phospho-Smad content, we do not have evidence of increased transcriptional activity and TGF- $\beta$ signaling under anoxia.

Looking at the protein expression of the co-Smad, Smad 4, it is apparent that in both tissues, the protein levels are unchanged between normoxia and anoxia. Again, while this does not support a profile of increased TGF- $\beta$ signaling activity in anoxia, it still indicates that there is certainly on-going TGF- $\beta$ signaling inside of anoxic cells. In the context of Smad regulation, Smad 4 is often targeted for nuclear expulsion followed by phosphorylation and/or sumoylation and then E3 ubiquitin ligase mediated degradation (Wu and Hill, 2009; Wrighton et al., 2009). 
Analysis of Smad 3 showed that transcript levels of a Smad 3-like protein increase in tail muscle under anoxia, while they do not change in the hepatopancreas. The protein levels correlate showing a strong increase in muscle with no change in hepatopancreas. However, when DNA binding studies were conducted for Smad 3, DNA binding activity was increased in hepatopancreas extracts from anoxic crayfish, whereas binding activity was unchanged in tail muscle. Smad 3, a receptor Smad, is only able to enter the nucleus when hetero-dimerized with Smad 2. The Smad 2/3 complex further complexes with the co-Smad (Smad 4), and is then able to move into the nucleus (Wu and Hill, 2009). Hence, although Smad 3 appears to be up regulated at both the transcript and protein levels in tail muscle under anoxia, it may in fact be more active under anoxia in the hepatopancreas as indicate by greater nuclear binding activity of Smad 3 and elevated amounts of phosphorylate (activated) Smad 2 in anoxic hepatopancreas. Smad activation profiling and potential mechanisms for control under anoxia

The Smad 2/3 complex is critical in TGF- $\beta$ signaling, and an increase in Smad 3 DNA binding activity would suggest the presence of this complex (in addition to Smad 4) in the nucleus. What is then interesting is that nuclear profiling of Smad 2-p in the nucleus remained level during anoxic exposure. This may outline two mechanisms of Smad activity during low oxygen stress. The first is that despite no significant increases in nuclear presence of Smad 2-p in the nucleus, through Smad 3 DNA binding profiles, Smad2 (which is in complex with 3) may also see increased DNA binding efficiency. This of course is largely speculative without analysis of alternate activating means, either by post-translational modification, or by binding to nuclear co-factors. In this line, it should be noted that the Smad 2/3/4 complex is known to be specifically aided by the 
TAZ transcriptional regulator, which helps with location to TGF- $\beta$ response elements; absence of TAZ can result in reduced nuclear location of the complex, and subsequent reduced transcriptional activity (Varelas et al., 2008). Hence, the presence or absence of TAZ may be the regulating factor controlling the Smad $2 / 3 / 4$ complex under anoxia, and may determine why there is no appreciable increase in nuclear Smad 2-p, despite an overall increase in Smad 2-p content. This may also explain the accumulation of Smad 2$\mathrm{p}$ in the cytoplasm. The issue of increased DNA binding by Smad 3 under anoxia may also be related to regulation through co-factors, in this case $\mathrm{p} 300 / \mathrm{CBP}$. The $\mathrm{p} 300 / \mathrm{CBP}$ co-activators contain histone acetyltransferase domains, and target lysine residues for acetylation (Inoue et al., 2007). Smad 3 can subsequently be regulated, particularly by acetylation of lysine 378 , to affect its transcriptional activity (Inoue et al., 2007). Hence the p300/CBP co-activators, and the level of their activity may provide the mechanism by which, despite probable unchanged expression of the Smad 2/3/4 complex in the nucleus, an increase in Smad 3 DNA binding activity can occur in the hepatopancreas under anoxia.

The second mechanism that may be outlined by the Smad profile may be a response dictated by the duration of oxygen limitation. In the hepatopancreas, although there are increases in phosphorylated Smads (both $1 / 5$ and 2), the critical marker of TGF$\beta$ signaling, Smad 2-p, does not appear to have an increased presence in the nucleus. This, along with the level profile of Smad 3 and 4 in the hepatopancreas may suggest a preparative mechanism of the cell, in anticipation of a return to normal levels of oxygen and aerobic metabolism. Indeed, in many models of metabolic rate depression, much of the activity during the stress period is preparatory for return to normal conditions, be it 
temperature, oxygen, moisture etc. Preparatory mechanisms are wide ranging; from upregulation of antioxidant defenses and detoxification proteins (see chapter 2 and HermesLima et al., 1998; Krivoruchko and Storey, 2010), to targets preventing muscle atrophy upon arousal (Tessier and Storey, 2010). The anticipatory up-regulation of the Smad proteins (including phosphorylated members), may be in order to prepare for a strong pro-developmental response with the return of a normal metabolic rate. By having phosphorylated Smads waiting in the cytoplasm, the cell would be able to quickly initiate growth and differentiation responses that would likely be necessary at the end of prolonged hypometabolism.

Of course this is partially tissue specific. While the majority of the data that would point to a preparatory mechanism is found in the hepatopancreas, the tail muscle tissue also appears to have altered Smad signaling, to the point of up-regulation. The hepatopancreas encompases liver and pancreas functions, and as such has a significantly higher rate of cell division, especially in comparison to skeletal muscle cells, so it may be expected that TGF- $\beta$ signaling in the hepatopancreas would be stronger; especially under normal metabolic rates. Nonetheless, transcript levels of a Smad 3-like protein, along with the total Smad 3 protein level rose under anoxia in the tail muscle, in conjunction with an increase in cytoplasmic Smad 2-p. While there was no increase in DNA binding capacity of Smad 3, along with no significant changes in nuclear Smad 2-p, there may be grounds to suggest that TGF- $\beta$ signaling in the muscle may be preparatory for recovery, and may or may not be controlled by differential activity of the Smad co-activators.

Regardless, the activation of the Smads under oxygen limitation is a complex process. TGF- $\beta$ signaling has previously been studied under hypoxic conditions $(<20 \%$ 
$\left.\mathrm{O}_{2}\right)$. Under hypoxic stress $\left(1 \% \mathrm{O}_{2}\right)$ it was seen that transcript levels of TGF- $\beta 2$ (the cytokine), as well as the type II receptor both increased in human endothelial cells (Akman et al., 2001). In the same models, hypoxia resulted in the phosphorylation and nuclear activation of Smad 2 and 3, resulting in the production (transcriptional) of the TGF- $\beta 2$ cytokine, in a feed forward response mechanism (Zhang et al., 2003). Certainly, the mechanism of Smad 2 phosphorylation, and increased transcriptional activity of Smad 3 hold true, possibly hinting at elements of a conserved response of TGF- $\beta$ signaling in response to oxygen deprivation.

STAT regulation in response to anoxia resistance

The STAT family of transcription factors also altered their activity under anoxia (Table 3.3). Protein levels of the STATs provide a less cohesive overview than the Smads, but this may in part be due to the differential and often conflicting roles that STAT transcription factors perform. Looking at the activation pattern, phosphorylation of tyrosine residues, there was a largely pro-STAT response. The relative amount of phosphorylated Stat 1 (Try 701) increased in both tissues under anoxia whereas phosphoStat 3 (Tyr 705) content increased in muscle. Only Stat 2-p content showed a significant reduction during anoxia in the hepatopancreas. Neither of the unphosphorylated members (Stat 5 and 6) studied changed their expression due to anoxic exposure. Again, like the Smads, this suggests that a proclivity to pro-development signaling remains even during anoxia.

What stands out among the protein data is the singular decrease in the prodevelopment transcription factors. The levels of phospho-Stat 2 drop approximately $60 \%$ under anoxia in the hepatopancreas. In the scope of the STAT family, Stat 2 is not as 
prominent as Stat 1 or 3, and its main role may not necessarily be in development, but in host immunity responses. To this effect, interferon (IFN) signaling (inducing JAK kinase) uses Stat 2-p, which complexes with Stat 1-p and an IFN regulatory factor family protein p48 - forming the ISGF3 complex (Bluyssen and Levy, 1997). Phosphorylated Stat 2 cannot bind alone to DNA (in complex with $\mathrm{p} 48$ ), and requires Stat 1-p in the complex to allow stable binding to DNA (Bluyssen and Levy, 1997). There is very little known about the crustacean immune response involving the STAT family, although in one model (the giant tiger prawn Penaeus monodon) STATs responded to infection (white spot syndrome virus), showing increased phospho-STAT levels and translocation into the nucleus (Chen et al., 2008). This however indicates that the immune response is triggered, as opposed to preparatory. Hence we may expect that the role of Stat 2-p could be minimized, and as such its decrease would not necessarily be detrimental to anoxia resistance.

Stat 1-p showed an interesting result. Transcript levels of a Stat 1-like protein increased significantly in muscle under anoxia, correlated with increased levels of Stat 1 phosphorylation. This phosphorylation, if increased transcript levels do indicate increased protein levels, would simply align with protein content, and not necessarily indicate an anoxia triggered post translational modification. Phosphorylation of Stat 1 also rose in the hepatopancreas under anoxia, but what is unique in these set of results is the decrease in nuclear Stat 1 (in both tissues) under anoxia as well as a decrease in DNA binding activity in the hepatopancreas. This appears to indicate that during anoxia, cells are suppressing the transcriptional activity of Stat 1 . Whether this (in conjunction with the suppression of Stat 2) and their role in an immune response complex is simply part of a 
metabolic reduction, is unknown. This may represent a reduction in protein synthesis or a mechanism to inhibit Stat 1's transcriptional targets. With regards to the latter, one avenue that is worth considering is the role of Stat 1 in apoptosis signaling. Stressadaptive animals such as crayfish often employ anti-apoptosis mechanisms as part of their survival strategy for long term survival of environmental stress. One part of this strategy would be to inhibit the transcription of pro-apoptotic genes, through inhibition of pro-apoptotic transcription factor. This is particularly relevant to Stat 1 since among its transcriptional targets are the death receptor, Fas and its ligand Fas-L, as well as caspase 1, a member of the protease family that is heavily involved in apoptosis cascades (Stephanou, 2004). Stat 1 also has inhibitory effects on the transcription of anti-apoptotic genes from the Bcl family, Bcl-2 and Bcl-xl (Stephanou, 2004). In conjunction with this, Stat 1 has multiple positive interactions with the tumor suppressor protein $\mathrm{p} 53$, which itself is heavily involved in apoptosis signaling. In the cytoplasm, Stat 1 is able to bind directly to $\mathrm{p} 53$, allowing the tumor suppressor to bind more efficiently to pro-apoptotic gene promoter sites (Stephanou and Latchman, 2003). Along with this, Stat 1 is a transcriptional repressor of the p53 inhibitor, MDM2 (Stephanou and Latchman, 2003), and thereby contributes to increasing p53 activity. Hence, the decrease in Stat 1-p nuclear presence under anoxia may be part of a cell preservation mechanism under anoxia. In relation to this, Stat 3 phosphoprotein content also increased under anoxia in the tail muscle. In terms of apoptotic signaling, Stat 3 opposes Stat 1, in that it is responsible for transcribing the anti-apoptotic Bcl-2 and Bcl-xl proteins that are repressed by Stat 1 (Stephanou and Latchman, 2003). To further the opposite nature of Stat 1 and 3 
expression, p53 also prevents Stat 3 phosphorylation, via a mechanism that has not been elucidated, which would prevent its anti-apoptotic role.

\section{Potential mechanisms of interrelation between Smad and Stat signaling}

While they are both the effectors of major cytokine-based developmental pathways, Smads and STATs appear to work in parallel as opposed to directly with each other. There is very little evidence for quantifiable direct interactions between the two families of transcription factors in $O$. virilis, but there are elements which warrant further research. In this line, one particular interaction of note is that of Stat 1 and 2 (in their ISGF3 complex - signaled by IFN) with the transcriptional activators p300/CBP (Bhattacharya et al., 1996). As mentioned earlier, these proteins are able to work in conjunction with the Smads, specifically Smad 3 to promote transcriptional activity. In the hepatopancreas, where there was decreased phosphorylation of Stat 2, reduced nuclear Stat 1-p and decreased DNA binding activity of Stat 1-p, we also see increased Smad 3 DNA binding activity. Though it may be unlikely, there is a possibility of a potential competitive nature between the ISGF3 complex and Smad 3 for p300. Given that there may not be a necessity for immune response during anoxia, the developmental protein may be given preferential binding.

In terms of working co-operatively, however, there are some speculated mechanisms that may support the notion of compensatory, or at least tandem, activity between these two major pro-development pathways. One such mechanism is repression of both Smads and STATs by the STAT inhibitor PIAS. In mammalian cells, PIASy (a PIAS family member) interacts strongly with Smad 3 and 4, repressing transcriptional activity - although not through DNA binding mechanisms (Long et al., 2003). The 
interaction between STAT inhibitor and Smad occurs most strongly in the presence of TGF- $\beta$ (Long et al., 2003), suggesting that this interaction occurs when conditions for growth and differentiation are optimal. This mechanism may point to a safety mechanism (from one developmental path to another) that is aimed at preventing dangerous levels of proliferation.

Finally, in the model organism C. elegans, STATs and Smads may exhibit a compensatory mechanism during the transition to the dauer stage whereby the STAT pathway can rescue TGF- $\beta$ (and Smad) signaling (see Appendix B.4).

\section{Conclusions}

Under anoxic conditions both Smads and STATs are regulated in $O$. virilis tail muscle and hepatopancreas. While the energetic dynamics during hypometabolism would suggest suppression of energetically costly processes, such as protein synthesis along with a general reduction in cell differentiation, the current data on pro-developmental transcription factors supports the idea of unchanged expression of developmental signals. These results (Table 3.3) generally accord with the hypothesis outlined above, and the hypothesis of the previous chapter. However, this subsequently conflicts with the idea of Smad regulation (and to a degree STAT regulation) seen in the dauer stage (see Appendix A), showing a deviation from a conserved response.

What is interesting are the cases of up-regulation that may indicate a preparatory mechanism to maintain a readiness for development upon return to normal, aerobic conditions. The activity profiles of nuclear entry for key members of the Smad and STAT families of transcription factors also suggest that while their total protein and phosphorylation levels can increase, this does not always manifest itself as increased 
transcriptional activity. There is also evidence that may suggest that these two major development pathways work in parallel, potentially overlapping in the use of key transcriptional co-factors, and certainly sharing some expression traits in a tissue specific manner between the tail muscle and hepatopancreas. Further studies indicating the relationship of complexing (Smad complexes and STAT complexes, separately) during anoxia would continue to elucidate both the regulation of the TGF- $\beta$ pathway and the JAK-STAT pathway, and may give a closer look at how development may be cooperatively regulated. 


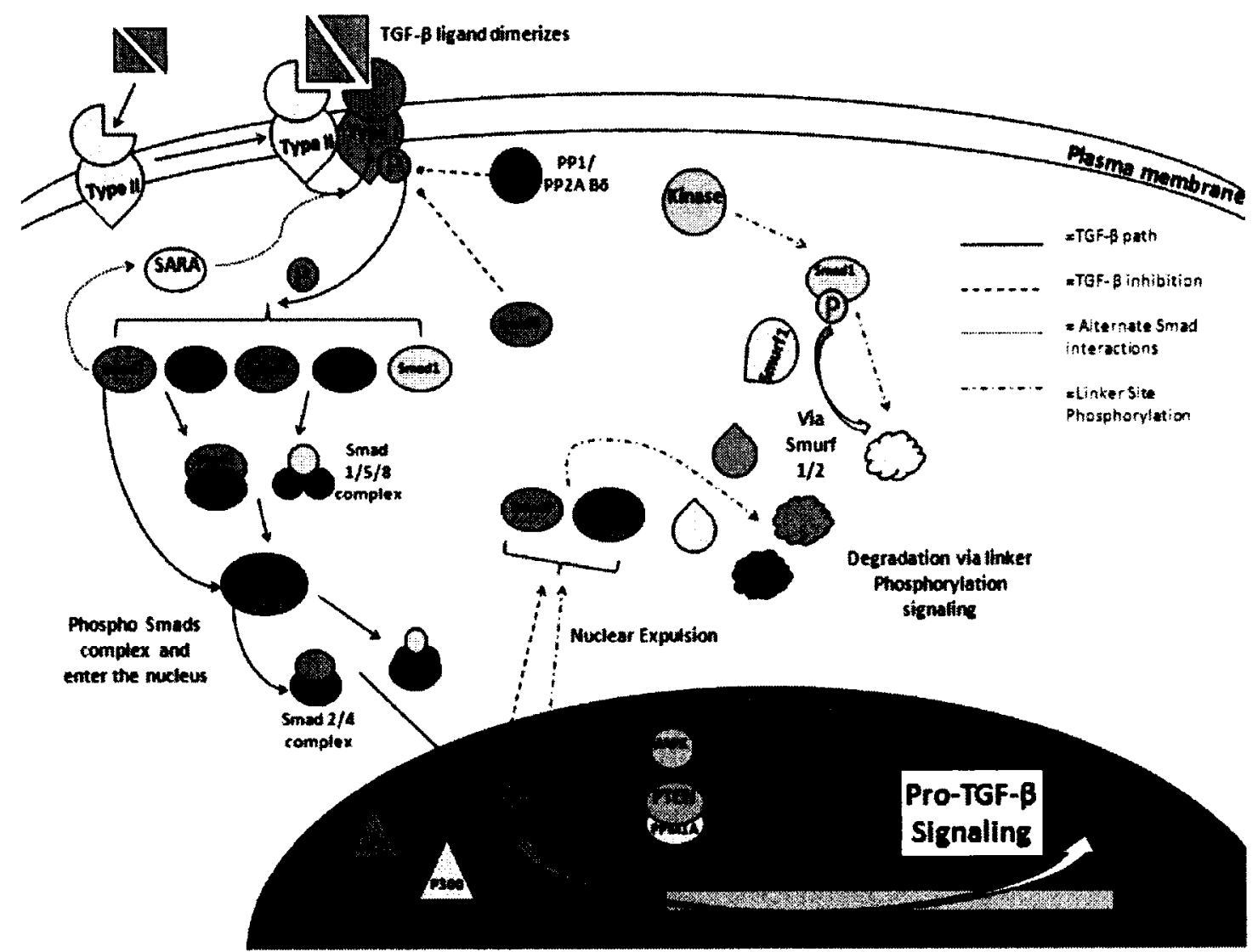

Figure 3.1: An overview of the TGF- $\beta$ pathway. In the presence of TGF- $\beta$, the pathway is activated. The two receptors (type I and II) interact to promote their own phosphorylation, and subsequently that of their downstream targets, the Smads. The rSmads, when phosphorylated, may complex together, and in conjunction with (co-)Smad 4 enter the nucleus. In the nucleus, a number of pro-developmental targets may be activated as a result of active Smads. Smad control is primarily through phosphorylation (negatively at the linker site), and they may also interact with co-factors in order to stabilize themselves. 


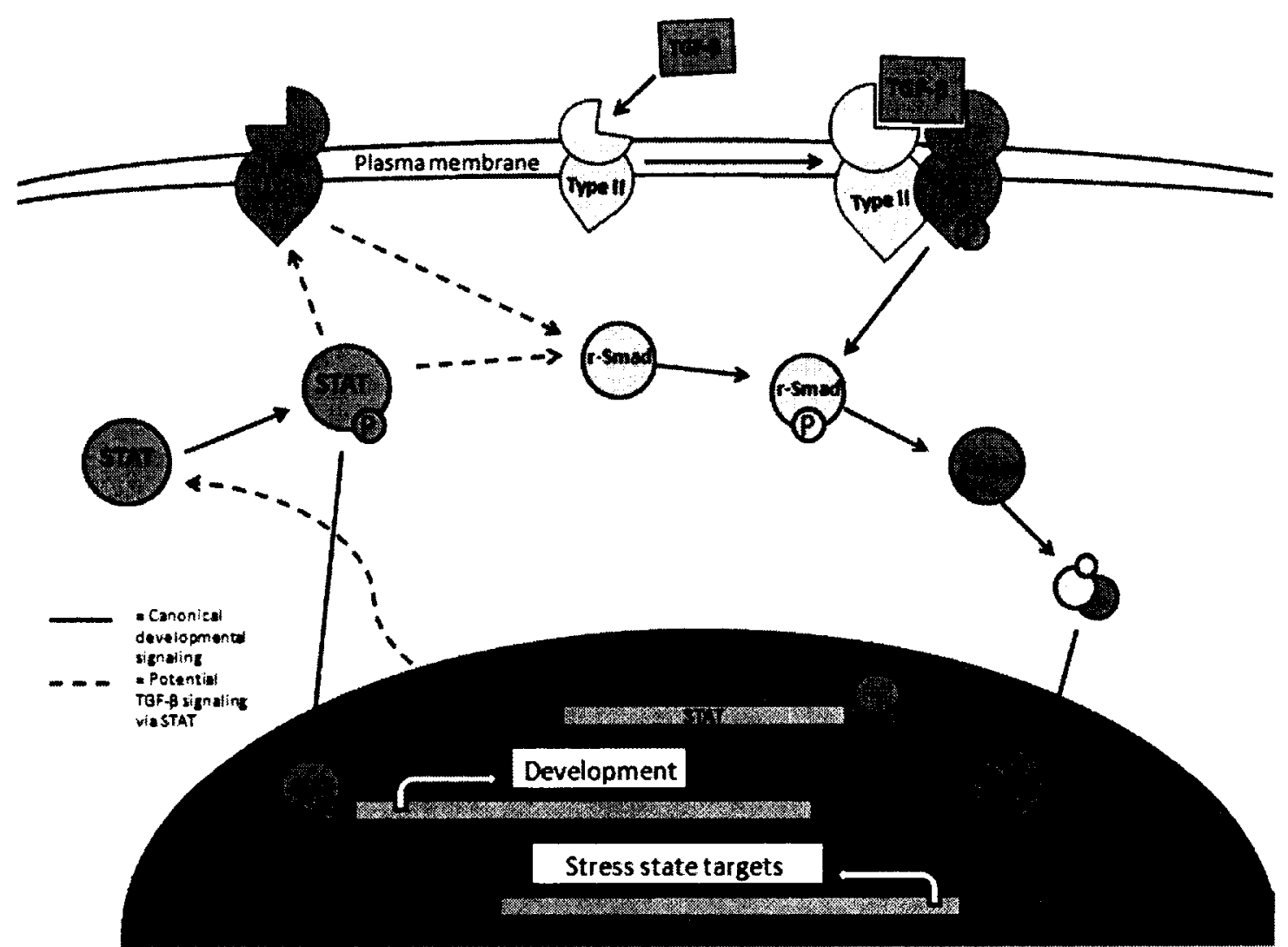

Figure 3.2: A potential mechanism for interaction between the pro-developmental TGF- $\beta$ and JAK-STAT pathways. Both pathways are largely pro-developmental, and there may be an overlap in regulation as implicated by the $C$. elegans dauer stage. A STAT transcription factor may be able to act in a compensatory mechanism given deficiencies in the TGF- $\beta$ mechanism, as well as be the target of anti-development path members acting through feedback inhibition. 

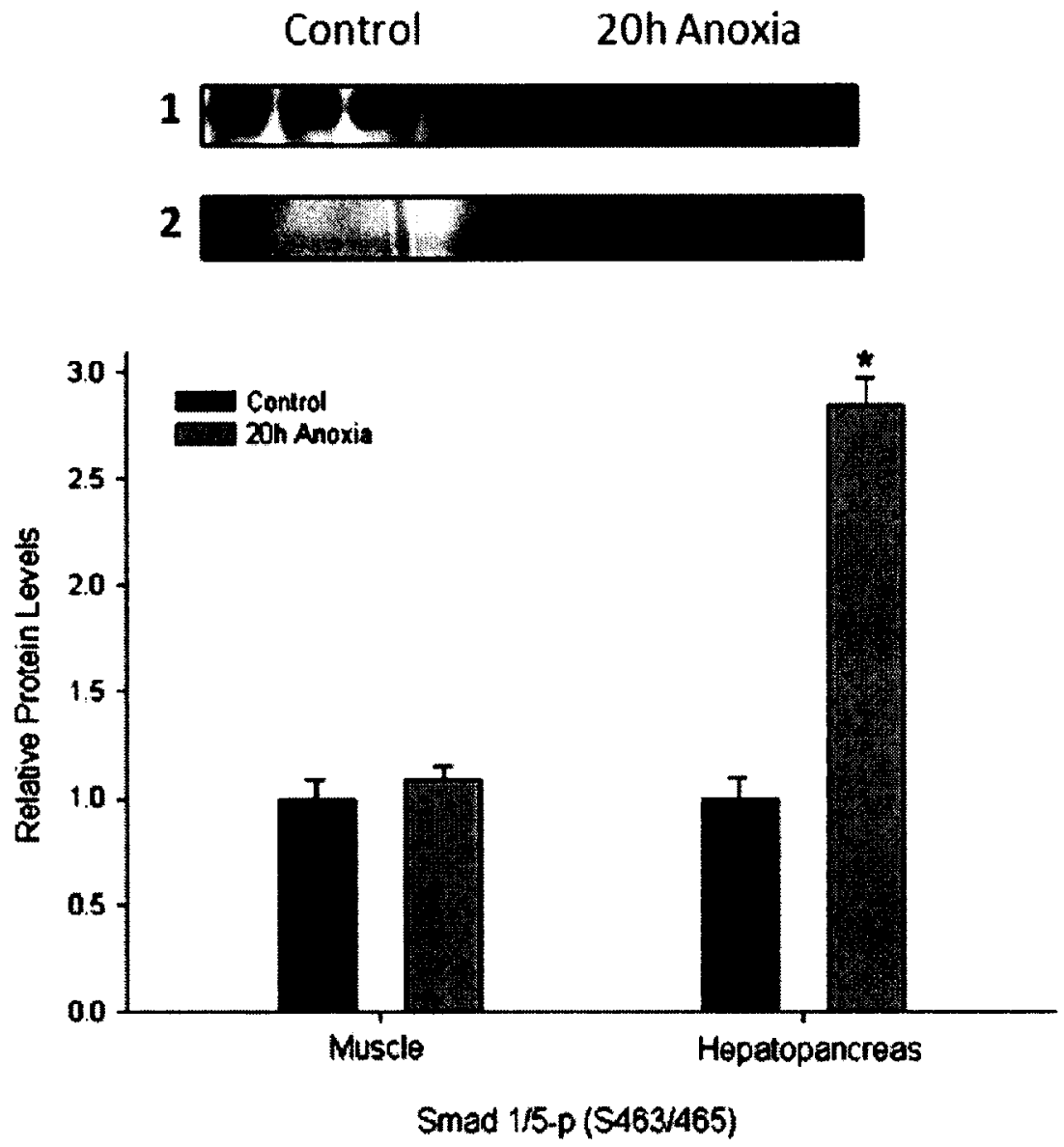

1

2

Figure 3.3: Effect of $20 \mathrm{~h}$ anoxic exposure on Smad 1/5-p activation in $O$. virilis tissues.

Changes in the relative levels of Smad 1/5-p (S463/465) phosphorylation in crayfish abdominal muscle and hepatopancreas in response to anoxia, as determined by western immunoblotting.

Data are means $\pm S E M, n=4$ independent trials for each tissue. ${ }^{*}$ indicates significantly different from the corresponding control, $P<0.05$. 

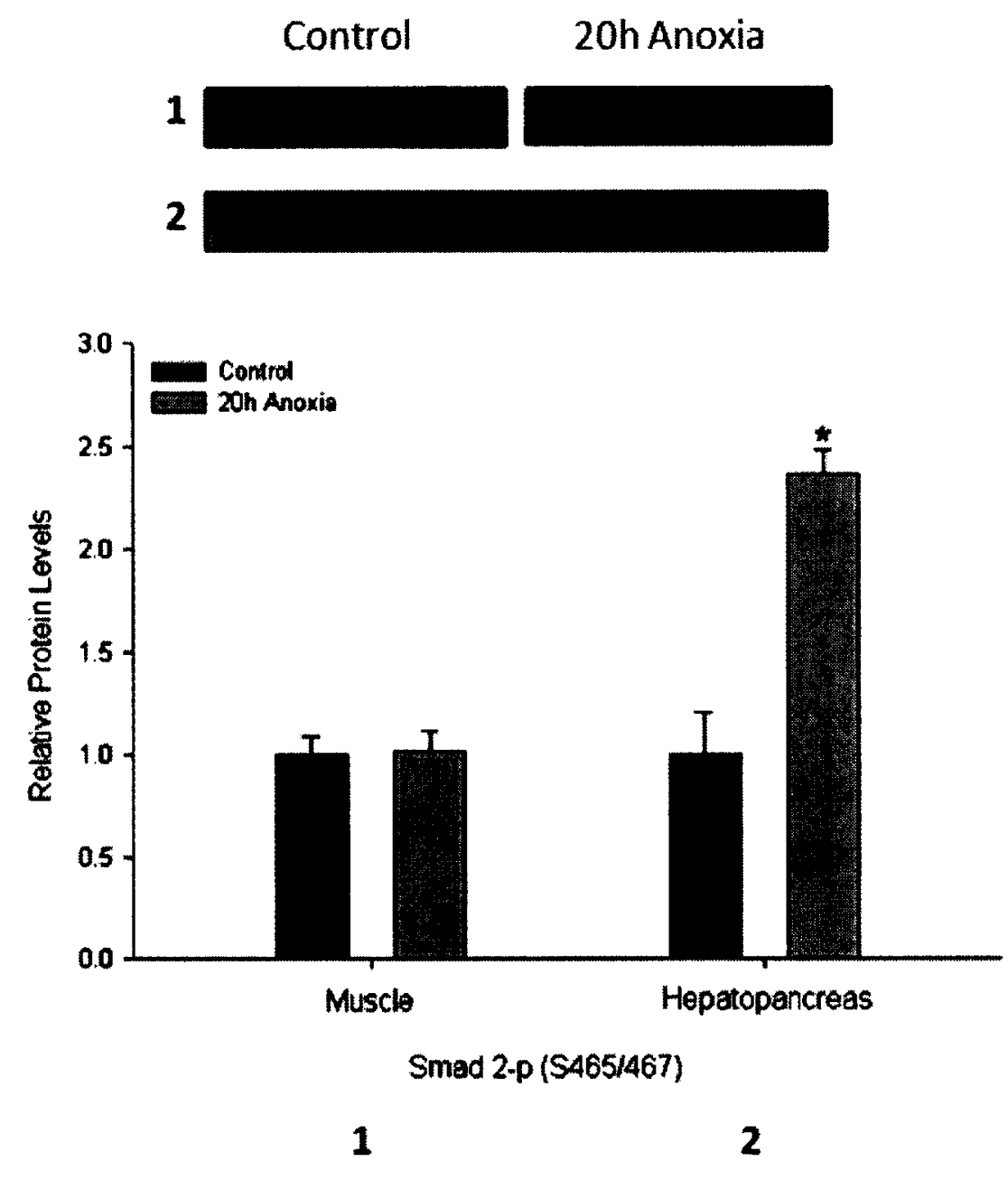

Figure 3.4: Effect of $20 \mathrm{~h}$ anoxic exposure on Smad 2-p activation in $\boldsymbol{O}$. virilis tissues. Changes in relative levels of Smad 2-p (S465/467) phosphorylation in crayfish tissues in response to anoxia, as determined by western immunoblotting.

Data are means $\pm S E M, n=4$ independent trials for muscle and hepatopancreas. * indicates significantly different from the corresponding control, $P<0.05$. 

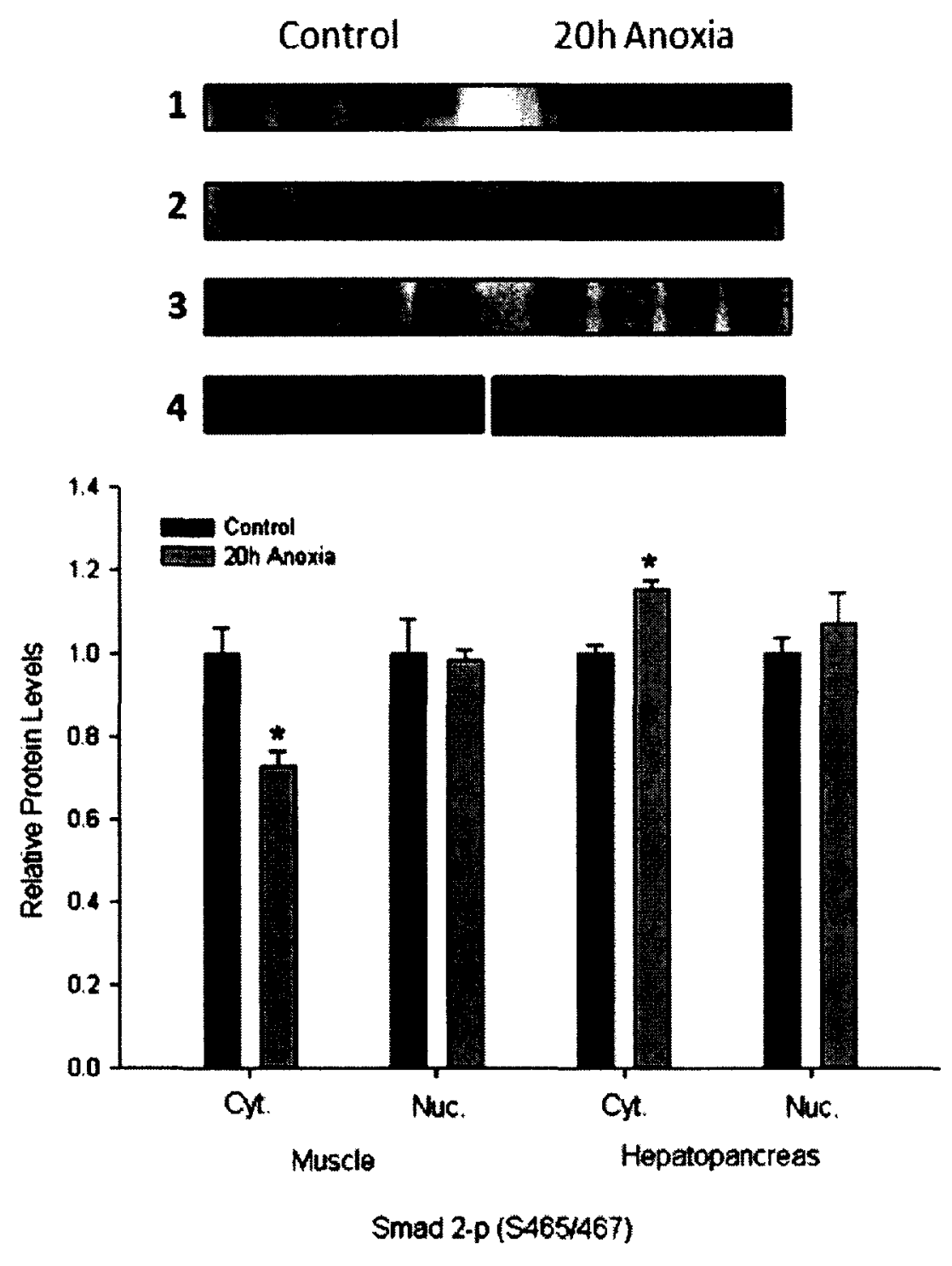

$\begin{array}{llll}1 & 2 & 3 & 4\end{array}$

Figure 3.5: Effect of $20 \mathrm{~h}$ anoxic exposure on Smad 2-p localization in O.virilis tissues.

Changes in relative levels of Smad 2-p (S465/467) phosphorylation in cytoplasmic and nuclear fractions of crayfish tissues in response to anoxia, as determined by western immunoblotting.

Data are means $\pm S E M, n=4$ independent trials for muscle and hepatopancreas. * indicates significantly different from the corresponding control, $P<0.05$. 
A)

1

1

61

21

121

41

181

61

B)

Water Flea: Crayfish identity $=42 \%$

535 CCCGCTGATTTAATGAATGTCAGCCGAGTGTCACCCAGCCCCCCCATCGACGCCCAACCG

1 CCCACTACTCTGAGAGTCTCTAAGAGTTAACATAAACAACCATTCATCGACTCTCTTCCA

595 GTTATGTACTGCGAACCGGCTTTTTGGTGTTCGATTAGCTATTATGAATTGAACACACGC

61 CTAATGGAACCATTACTGGCCATTTGGTGTACCATCACCCGTCTGGATTGACCAGTACT

655 GTGGGAGAAACTTTTCATGCATCTCAGCCATCCATTACCGTTGACGGTTTTACTGATCCC

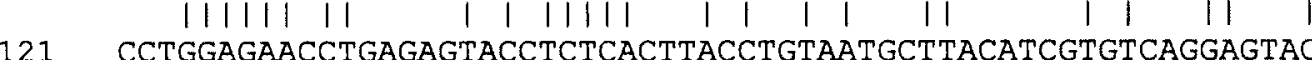

715 AGCAACTCGGAGCGGTTTTGCCTGGGGCTGTTATCAAACGTGAATCGTAATCCTGTTGTG

181 I I I I I I I

C)
CCCACTACTCTGAGAGTCTCTAAGAGTTAACATAAACAACCATTCATCGACTCTCTTCCA

CTAATGGAACCATTACTGGCCATTTGGTGTACCCATCACCCGTCTGGATTGACCAGTACT

CCTGGAGAACCTGAGAGTACCTCTCACTTACCTGTAATGCTTACATCGTGTCAGGAGTAC

ATATTACCCACCTAGCACCATCTGGAGTACAGACGACAGACAG

$\begin{array}{lllllllllllllll}I & \text { L } & \text { P } & \text { T } & \text { H } & \text { H } & \text { L } & \text { E } & \text { Y } & \text { R } & \text { R } & \mathbf{Q} & \mathbf{T}\end{array}$

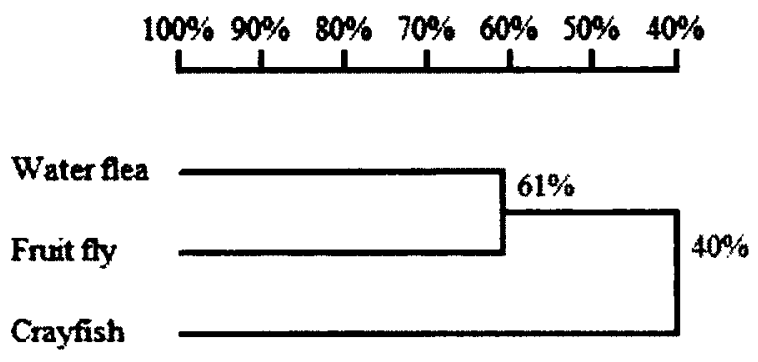

Figure 3.6: Partial nucleotide and deduced amino acid sequence of $O$. virilis Smad 3like protein.

(A) The partial cDNA sequence of a Smad 3-like protein, with the corresponding amino acid sequence is shown. Nucleotides and amino acids are numbered on the left. The nucleotide sequence was 166 nucleotides in length, and encoded 52 amino acids.

(B) Sequence alignment of the isolated crayfish sequence and the corresponding fragment of the water flea sequence. 
(C) Homology tree produced from an alignment of the partial $O$. virilis Smad 3-like protein sequence with Smad 3 from fruit fly (Drosophila melanogaster) and water flea (Daphnia pulex). The accession number for the fruit fly is NM_057669. There are currently no accession numbers for $D$. pulex genes, but sequences may be found at http://wfleabase.org/. The percentage values correspond to the shared identity among the corresponding species. 

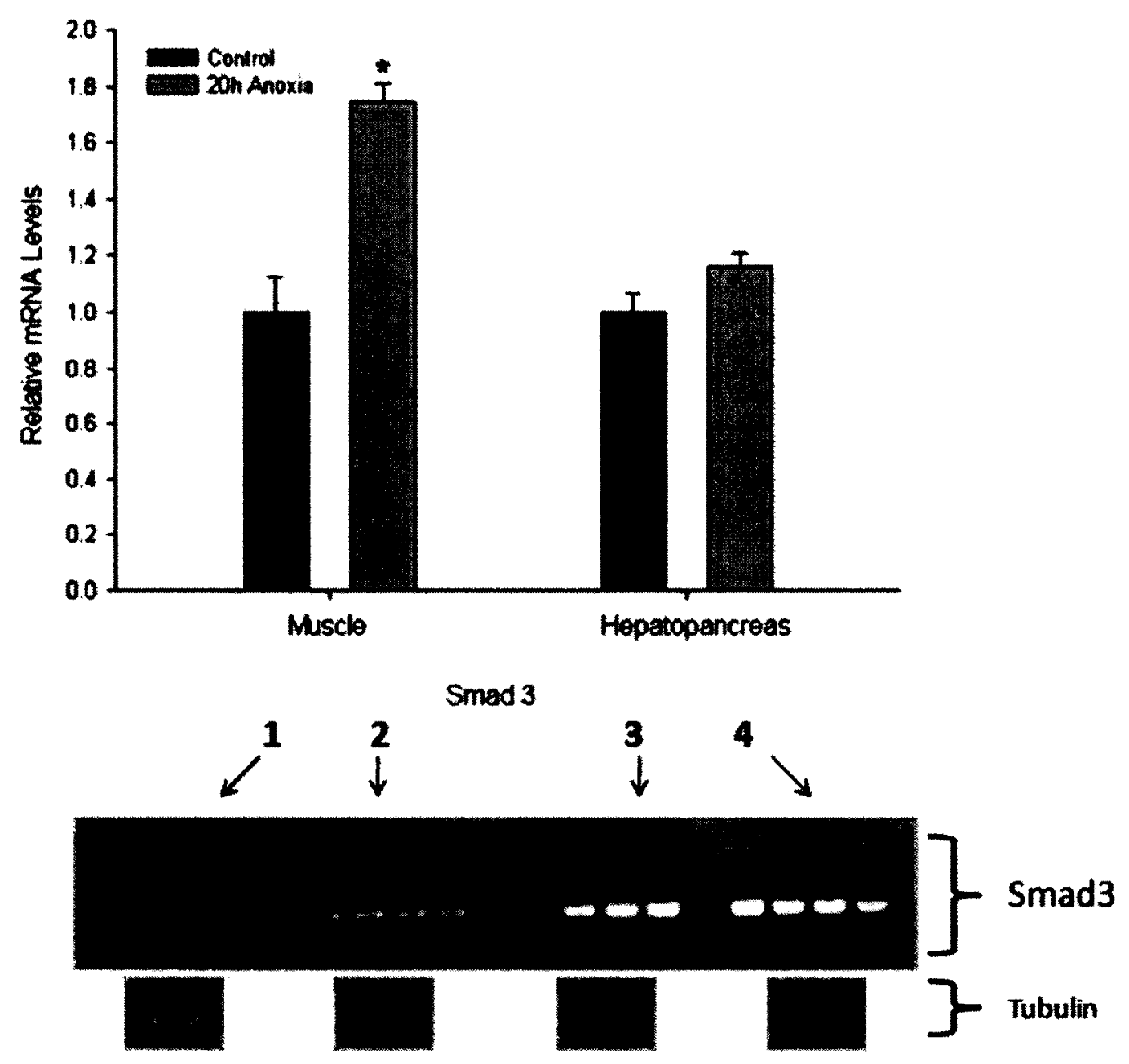

Figure3.7: Effect of $20 \mathrm{~h}$ anoxic exposure on Smad 3-like transcript expression in $O$. virilis tissues as determined by RT-PCR.

Histogram shows the normalized Smad 3-like transcript levels under control and anoxic conditions; bands were normalized against the corresponding $\alpha$-tubulin bands (representative from each sample shown) amplified from the same samples. Data are means $\pm \mathrm{SEM}, n=4$ independent trials for mRNA isolated from different crayfish. * Indicates significantly different from the corresponding control, $P<0.05$.

$1=$ control muscle, $2=20 \mathrm{~h}$ anoxic muscle, $3=$ control hepatopancreas, $4=20 \mathrm{~h}$ anoxic hepatopancreas

Representative Smad 3 amplicons are also shown. 


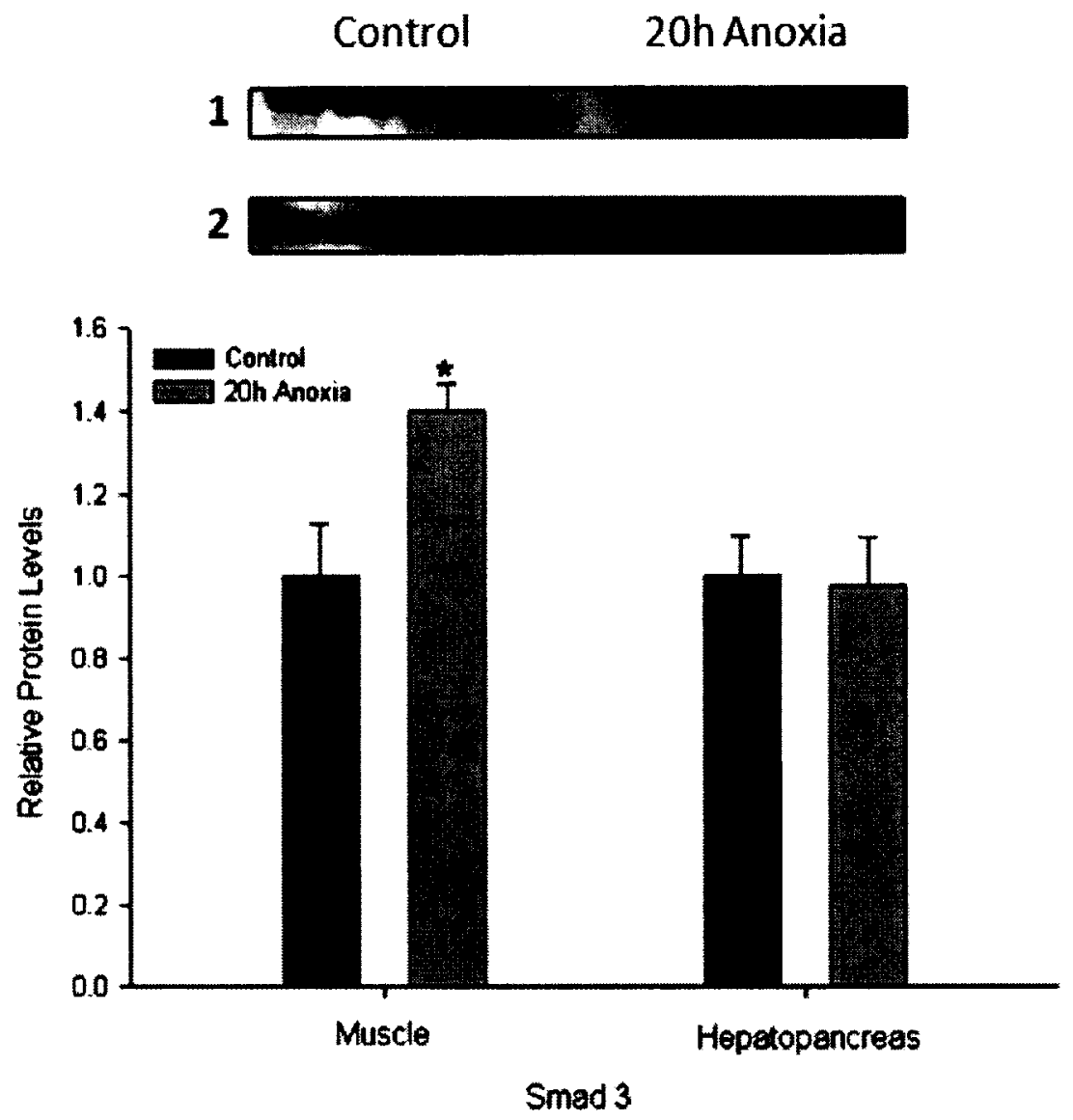

1

Figure 3.8: Effect of $20 \mathrm{~h}$ anoxic exposure on Smad 3 levels in $O$.virilis tissues.

Changes in Smad 3 protein levels in crayfish tissues in response to anoxia, as determined by western immunoblotting.

Data are means \pm SEM, $n=4$ independent trials for muscle and hepatopancreas. * indicates significantly different from the corresponding control, $P<0.05$. 

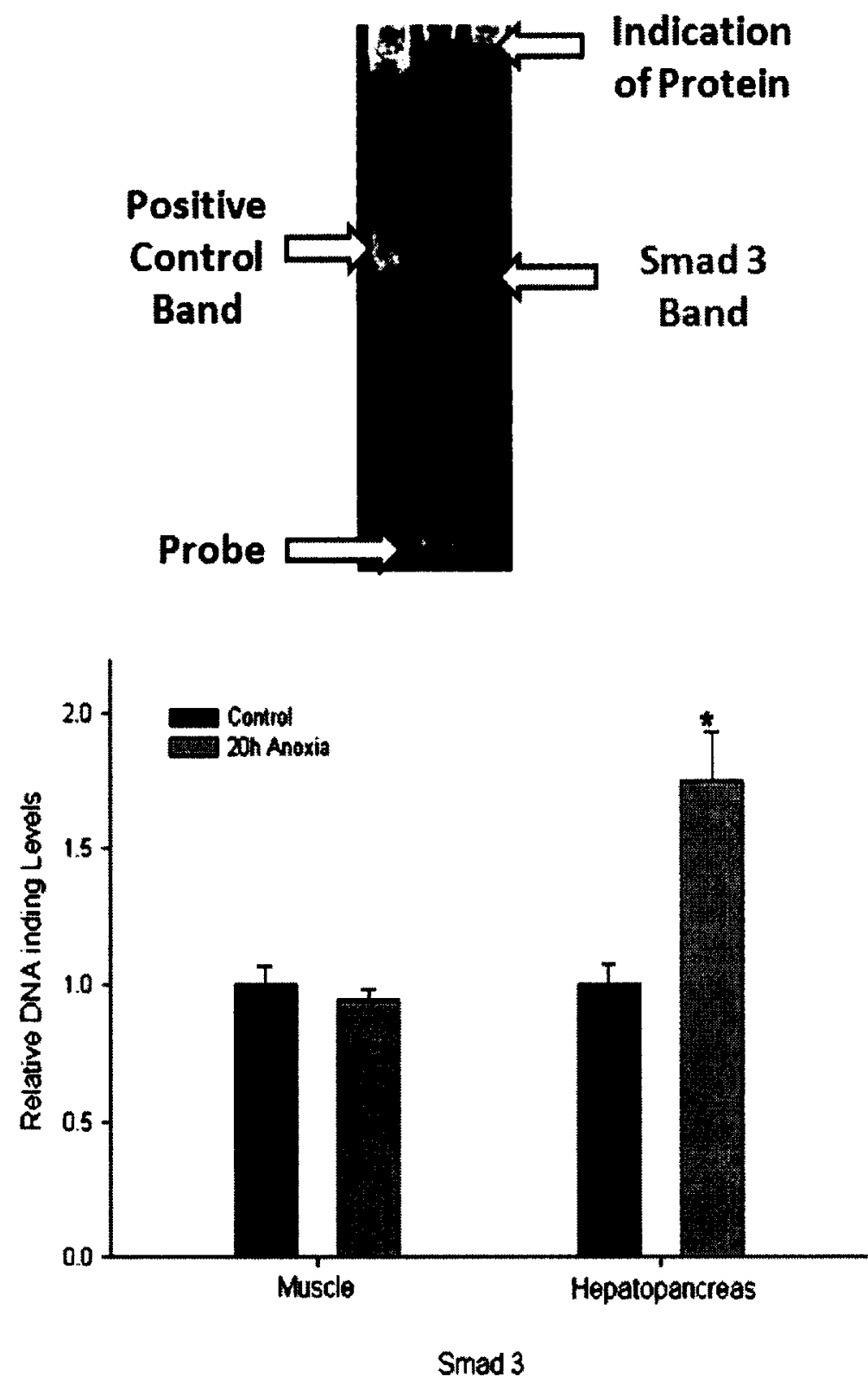

Figure 3.9: Effect of $20 \mathrm{~h}$ anoxic submergence on Smad 3 activity in $O$. virilis tissues. Changes in the DNA-binding activity of nuclear Smad 3 in the muscle and hepatopancreas in response to anoxia, as determined by a transcription factor binding assay. Data are means $\pm \mathrm{SEM}, n=4$ independent trials. ${ }^{*}$ Indicates significantly different from the corresponding control, $P<0.05$.

A representative EMSA is shown above the histogram. 


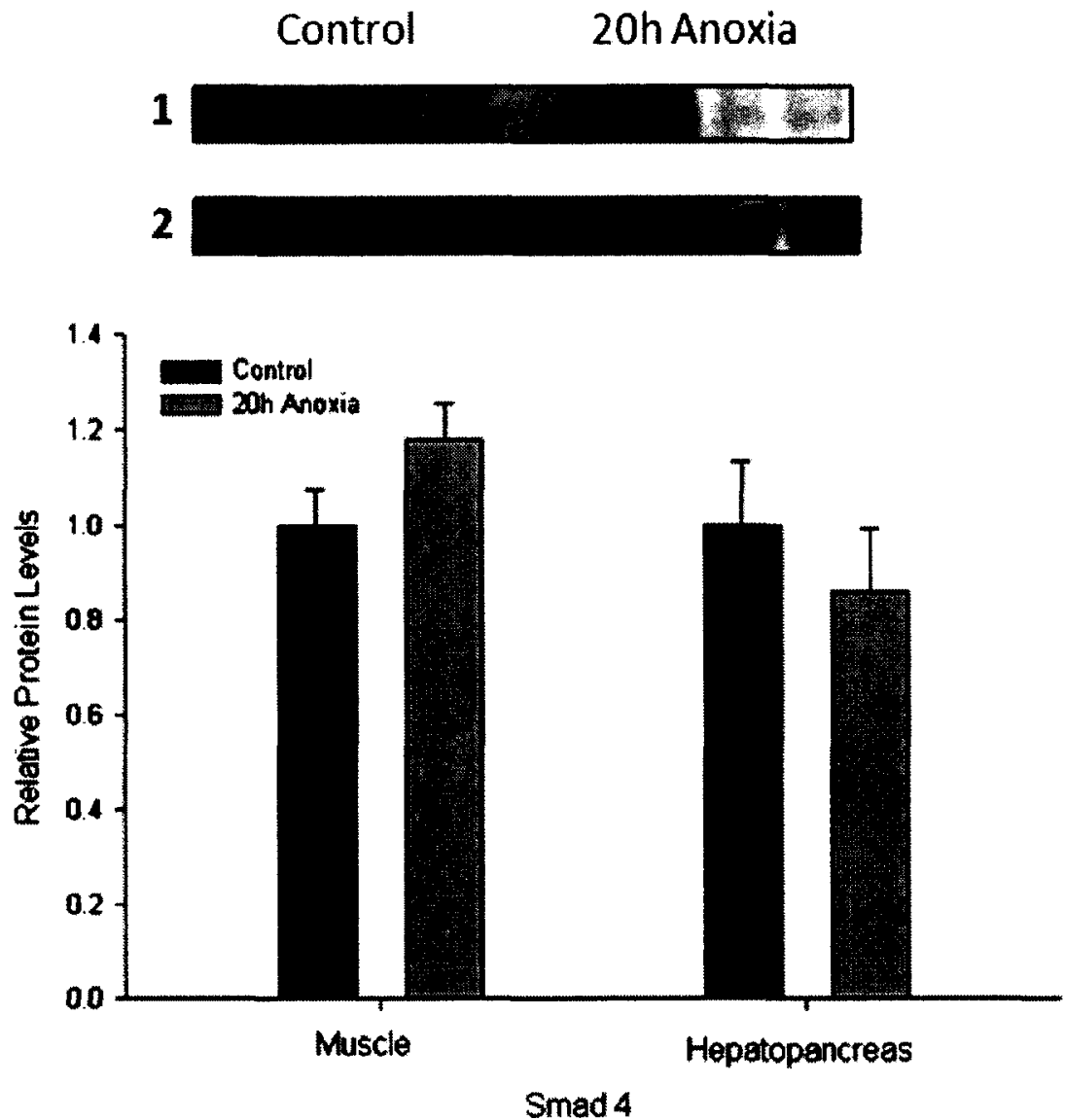

Figure 3.10: Effect of $20 \mathrm{~h}$ anoxic exposure on Smad 4 levels in $O$.virilis tissues. Changes in Smad 4 protein levels in crayfish tissues in response to anoxia, as determined by western immunoblotting.

Data are means \pm SEM, $\mathrm{n}=4$ independent trials for muscle and hepatopancreas. * indicates significantly different from the corresponding control, $P<0.05$. 


\begin{abstract}
A)
1

1

61

21

121

41

181

61

241

81

301

101

TCTCGATGCGAGCACGGGCACGAGCTGTTACTCGTAGTGCACATACTGTAAGCCTTTGGG

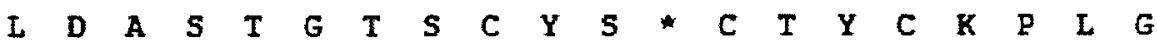
ACTTGAAGAGGCGCAGGTCATCAGT GACGGTGCCAACAACACACACAATCCTCCCCTGGC $\begin{array}{lllllllllllllllllllll}\text { L } & \mathbf{E} & \mathbf{E} & \mathbf{A} & \mathbf{Q} & \mathbf{V} & \mathbf{I} & \mathbf{S} & \mathbf{D} & \mathbf{G} & \mathbf{A} & \mathbf{N} & \mathbf{N} & \mathbf{T} & \mathbf{H} & \mathbf{N} & \mathbf{P} & \mathbf{P} & \mathbf{L} & \mathbf{A}\end{array}$ GGCCAGGCTITCTAGCAAGTTGCACGAGGCGAGATAGAGAGAGAGGGGGGTGATGAATGC $\begin{array}{llllllllllllllllllll}A & R & I & S & S & K & L & H & E & A & R & * & R & E & R & G & V & M & N & A\end{array}$ AAAACATGAACAATCTCTTCAGAATGACCTTGTTAAACTTGGAGTTGGTGCGGCGTGCCA $\begin{array}{llllllllllllllllllll}K & \text { H } & \mathbf{E} & \mathbf{Q} & \mathbf{S} & \text { L } & \mathbf{Q} & \text { N } & \text { D } & \text { L } & \text { V } & \text { K } & \text { L } & \text { G } & \text { V } & \text { G } & \text { A } & \text { A } & \text { C } & Q\end{array}$ AAAAGCGGTAGAGCTTCACCAGAAGCCTCAGGTAAACATCCTGAGACTTGGGCTCCCGCC

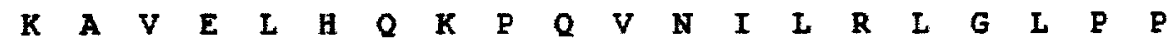
TGATGACTTTTCGGTCGTGTTTGTGAGAGATATCCTTACCCATAGCAACCTCGACTCTAA $\begin{array}{lllllllllllllllllll}D & D & F & S & V & V & F & V & R & D & I & I & I & H & S & N & I & D & S\end{array}$
\end{abstract}

B)

\begin{tabular}{|c|c|}
\hline 44 & GAAACCGGCGAAAATTTGAGACAAATGGAACAGGAACACGAAGCCTTTGCCTTA.GAATA \\
\hline & 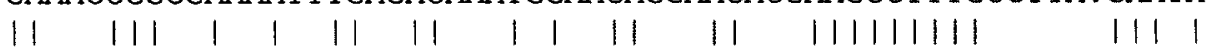 \\
\hline 10 & GAGCACGGGCACGAGCTGTTACTCGTAGTGCACATACTGTAAGCCTTTGGGACTTGAAGA \\
\hline 3 & TCATGAGCTAACTAAAGTGCAAGCCAGCATTCAACACGTACAAAACAATCCACTCACGCA \\
\hline & 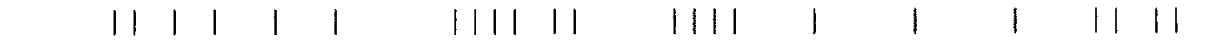 \\
\hline 0 & GGCGCAGGTCATCAGTGACGGTGCCAACAACACACACAATCCTCCCCTGGCGGCCAGGCT \\
\hline 53 & GTCACCGGACGTGGAAAAACAGCTAAGGGCAAAGAAGGATTCTCTGGCTCAAAAAGTCAA \\
\hline & 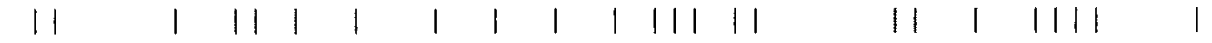 \\
\hline 30 & TTCTAGCAAGTTGCACGAGGCGAGATAGAGAGAGAGGGGGGTGATGAATGCAAAACATGA \\
\hline 23 & TCAAAGAGTGGTTGAGTTACTTAAAACACGTGCACTTCTGGCCGAAAAACACAAAGACAC \\
\hline & $\begin{array}{llllllllll}1 & 11 & 1 & 11 & 11 & 1 & 11 & 11 & 1\end{array}$ \\
\hline 90 & ACAATCTCTTCAGAATGACCTTGTTAAACTTGGAGTTGGTGCGGCGTGCCAAAAAGCGGT \\
\hline 83 & CGTAGTTGGTATTGGCAGTCTCCAAGGACGGGTCCTTGACGATGAACTAATACGCTGGAA \\
\hline & 111111111111 \\
\hline 50 & AGAGCTTCAC . . . . CAGAAGCCTCAGGTAAACATCCTGAGACTTGGGCTCCCGCCTGAT \\
\hline 343 & GAGAGAACAACAACTAGCTGGAAAT \\
\hline & 111 \\
\hline
\end{tabular}


C)
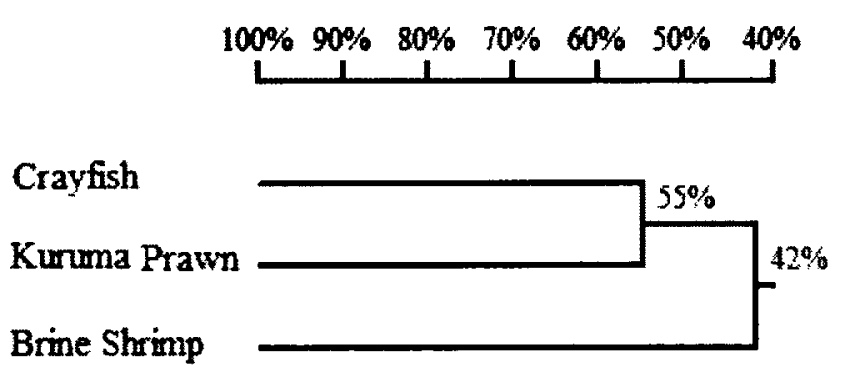

Figure 3.11: Partial nucleotide and deduced amino acid sequence of $O$. virilis Stat 1like protein.

(A) The partial cDNA sequence of a Stat 1 -like protein, with the corresponding amino acid sequence is shown. Nucleotides and amino acids are numbered on the left. The nucleotide sequence was 361 nucleotides in length, and encoded 110 amino acids.

(B) Sequence alignment of the isolated crayfish sequence and the corresponding fragment of the brine shrimp sequence.

(C) Homology tree produced from an alignment of the partial $O$. virilis Stat 1-like cDNA sequence with Stat 1 from the kuruma prawn (Marsupenaeus japonicus) and the brine shrimp (Artemia franciscana). Accession numbers are BAI49681.1 for the kuruma prawn and ACJ63721.1 for the brine shrimp. The percentage values correspond to the shared identity among the corresponding species. 


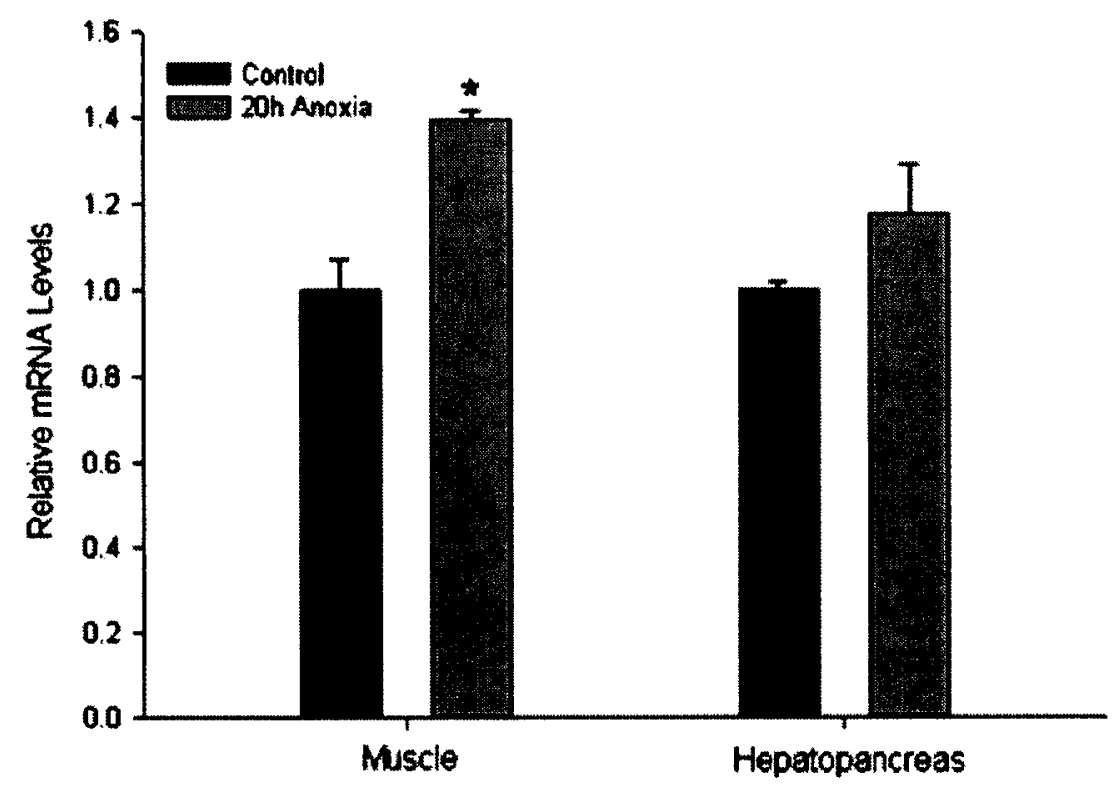

STAT 1

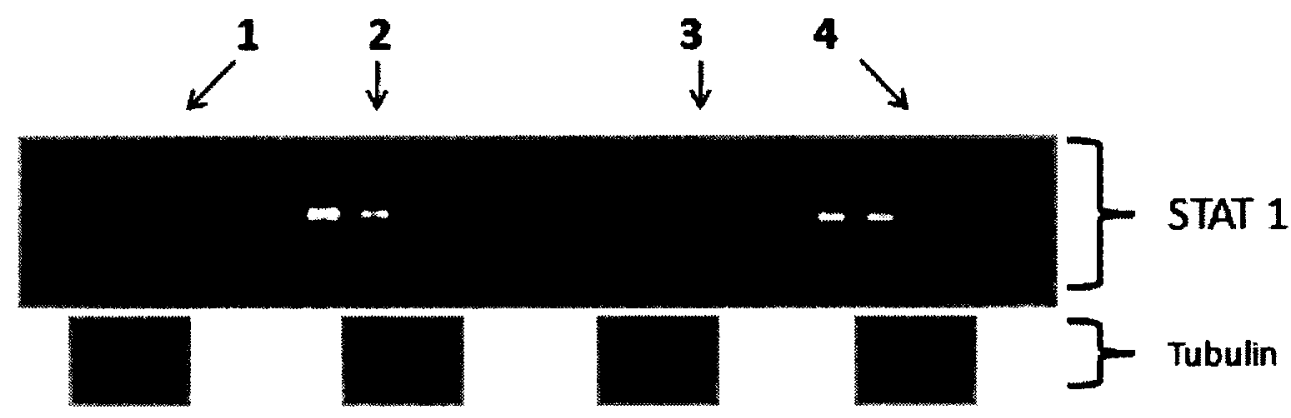

Figure 3.12: Effect of $20 \mathrm{~h}$ anoxic exposure on Stat-1 -like transcript expression in $\boldsymbol{O}$. virilis tissues as determined by RT-PCR.

Histogram shows the normalized transcript levels under control and anoxic conditions; bands were normalized against the corresponding $\alpha$-tubulin bands (representative from each sample shown) amplified from the same sample. Data are means \pm SEM, $n=4$ independent trials for $\mathrm{mRNA}$ isolated from different crayfish. ${ }^{*}$ Indicates significantly different from the corresponding control, $P<0.05$.

$1=$ control muscle, $2=20 \mathrm{~h}$ anoxic muscle, $3=$ control hepatopancreas, $4=20 \mathrm{~h}$ anoxic hepatopancreas

Representative Stat 1 amplicons shown below. 

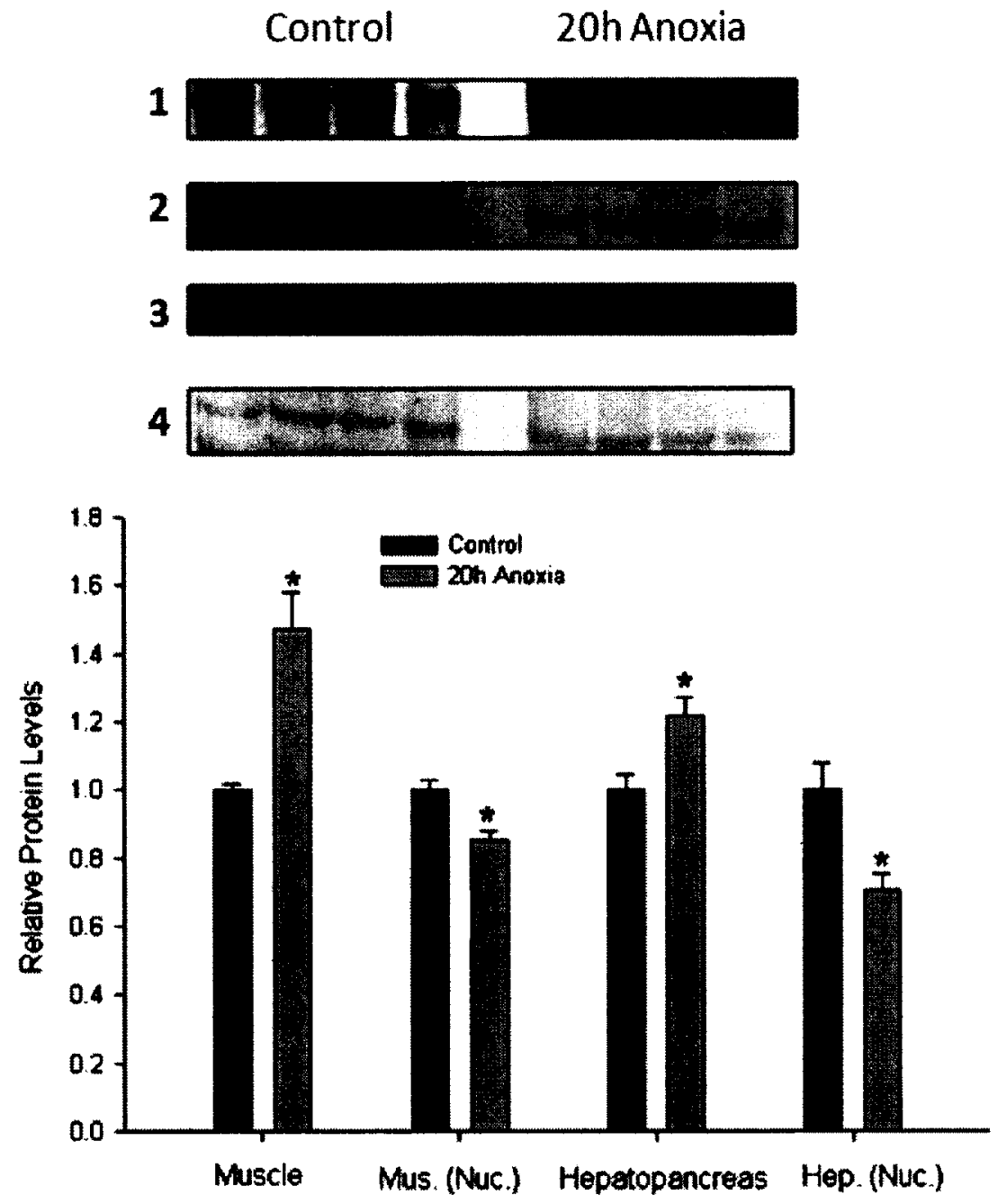

Stat 1-p (T701)

$1 \quad 2 \quad 3 \quad 4$

Figure 3.13: Effect of $20 \mathrm{~h}$ anoxic exposure on Stat 1-p activation in 0 .virilis tissues. Changes in the relative levels of Stat 1-p (T701) phosphorylation in total and nuclear fractions of crayfish tissues in response to anoxia, as determined by western immunoblotting.

Data are means $\pm S E M, n=4$ independent trials for muscle and hepatopancreas. * indicates significantly different from the corresponding control, $P<0.05$. 

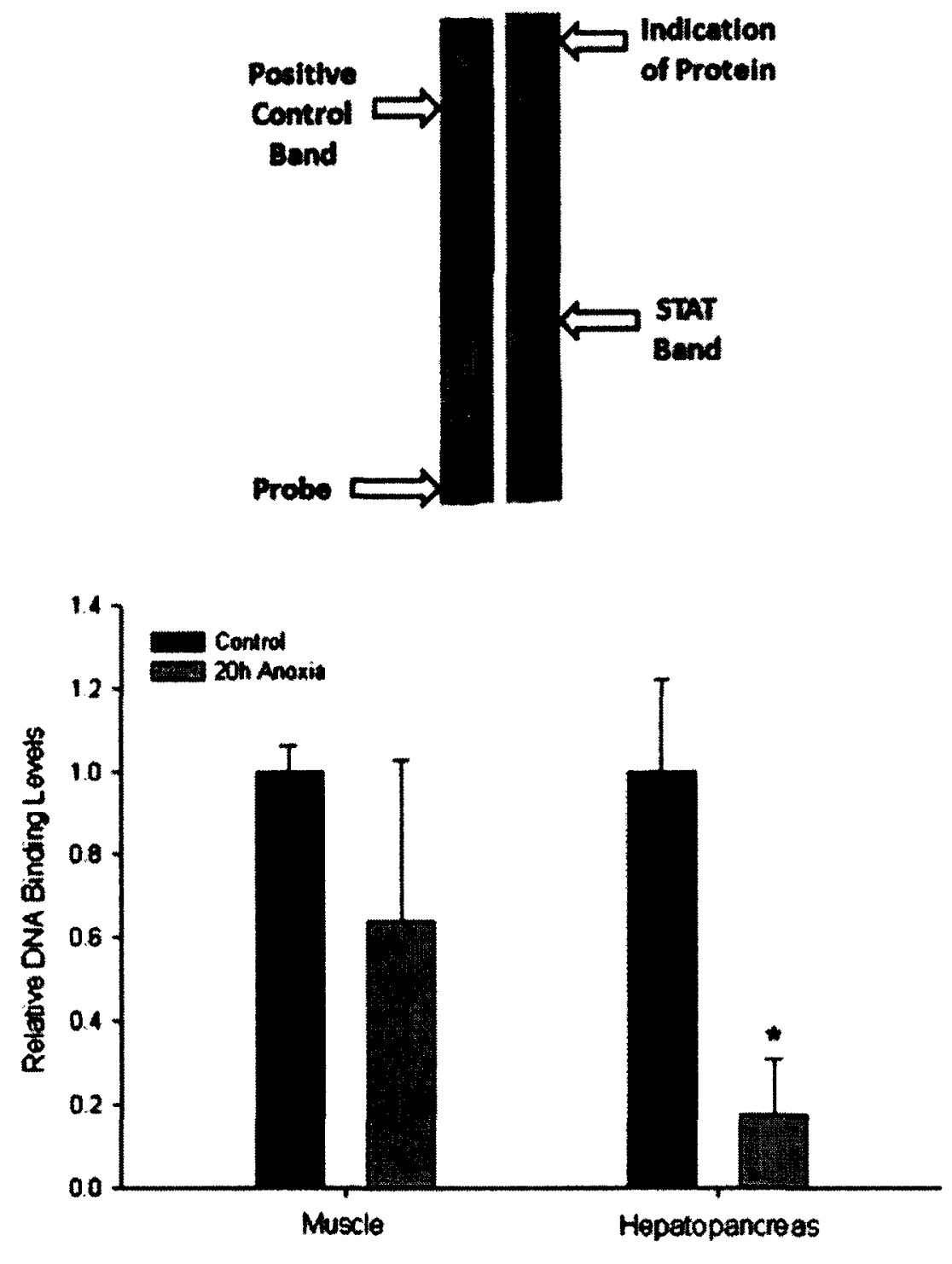

STAT 1

Figure 3.14: Effect of $20 \mathrm{~h}$ anoxic submergence on Stat 1 activity in $O$. virilis tissues. Changes in the DNA-binding activity of nuclear Stat 1 in the muscle and hepatopancreas in response to anoxia, as determined by a transcription factor binding assay. Data are means \pm SEM, $n=4$ independent trials. * Indicates significantly different from the corresponding control, $P<0.05$. 


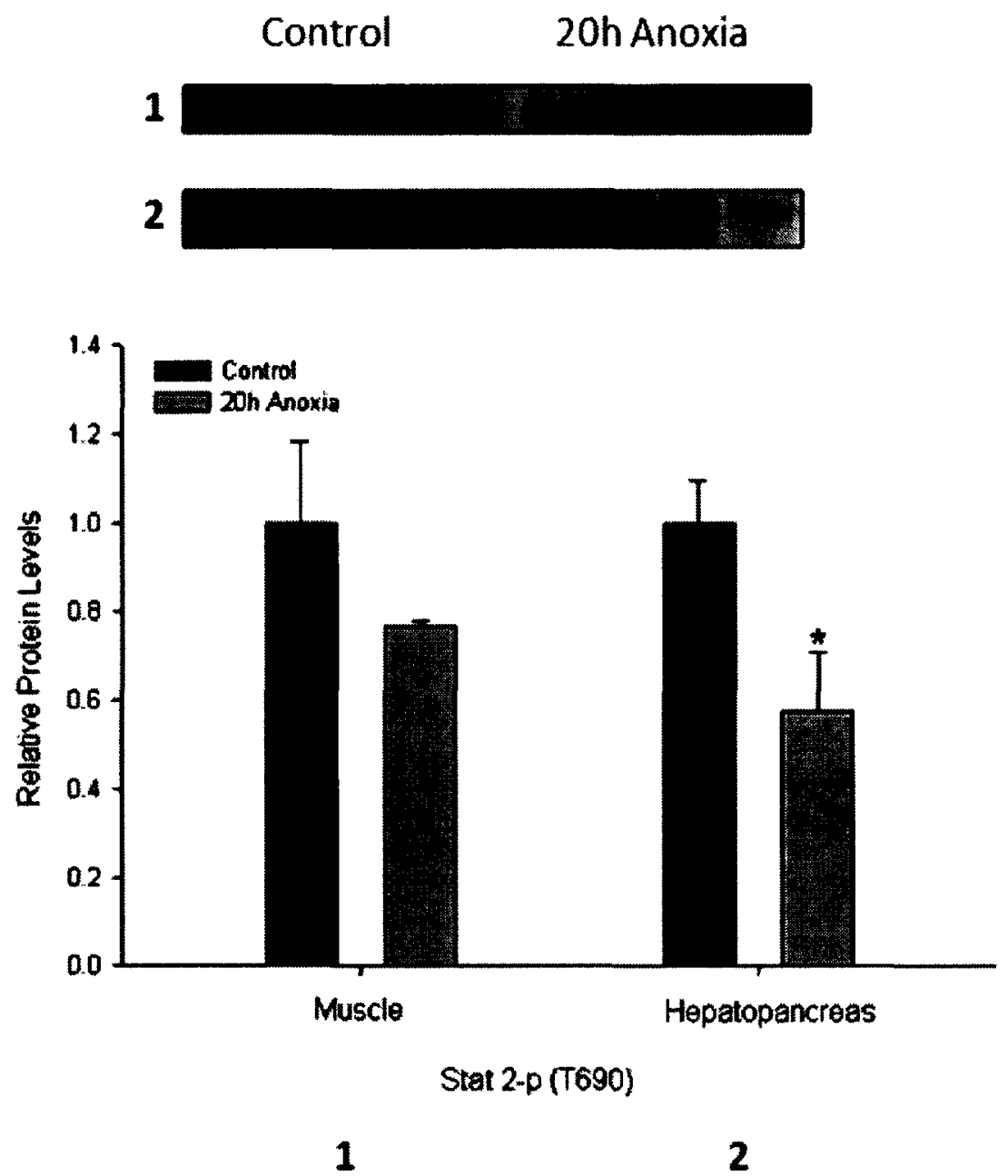

Figure 3.15: Effect of $20 \mathrm{~h}$ anoxic exposure on Stat 2-p activation in $O$. virilis tissues. Changes in the relative Stat 2-p (T690) phosphorylation in crayfish tissues in response to anoxia, as determined by western immunoblotting. Data are means $\pm S E M, n=4$ independent trials for muscle and hepatopancreas. * indicates significantly different from the corresponding control, $P<0.05$. 


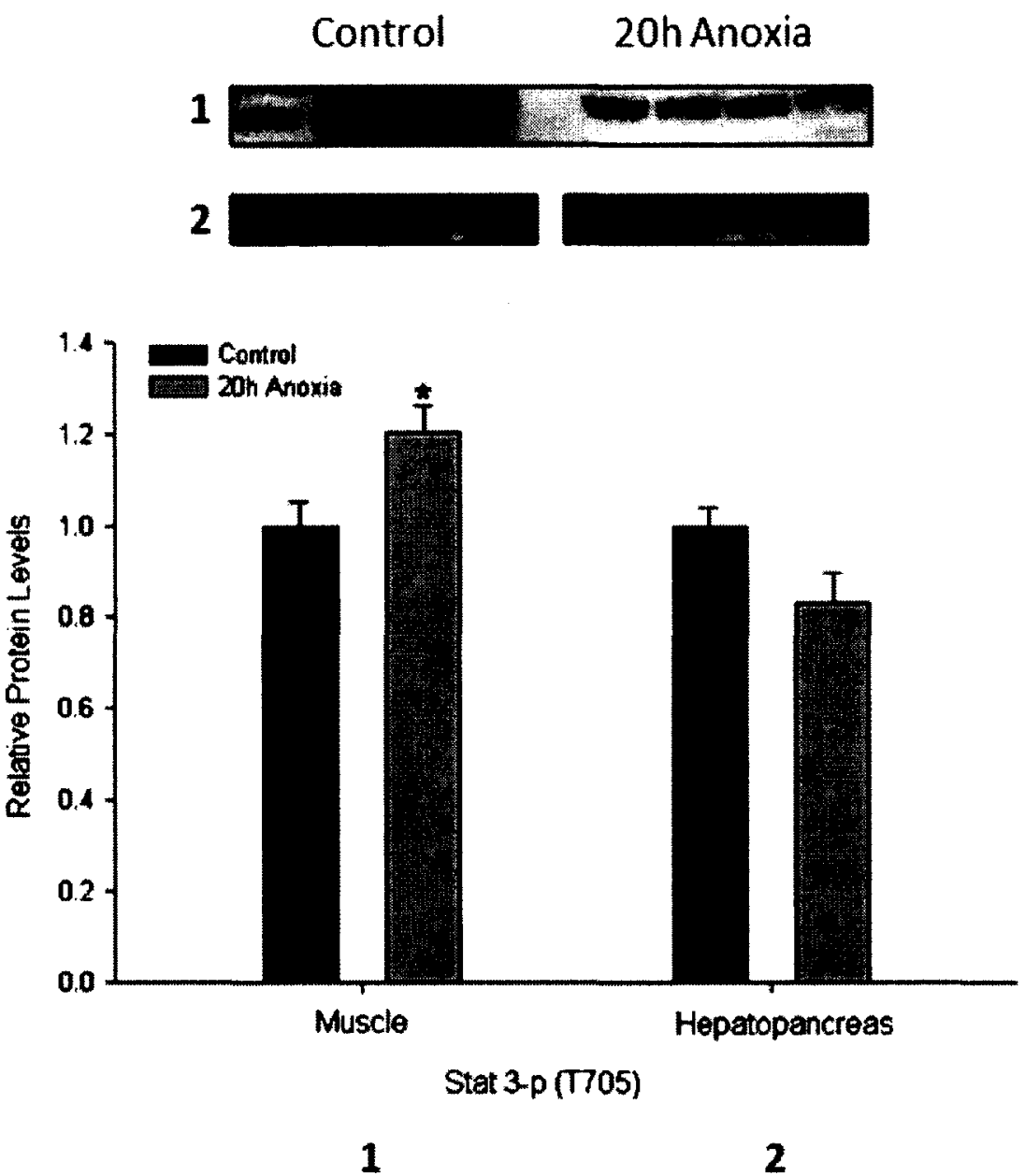

Figure 3.16: Effect of $20 \mathrm{~h}$ anoxic exposure on Stat 3-p activation in $O$.virilis tissues. Changes in the relative levels of Stat 3-p (T705) phosphorylation in crayfish tissues in response to anoxia, as determined by western immunoblotting.

Data are means \pm SEM, $\mathrm{n}=4$ independent trials for muscle and hepatopancreas. * indicates significantly different from the corresponding control, $P<0.05$. 

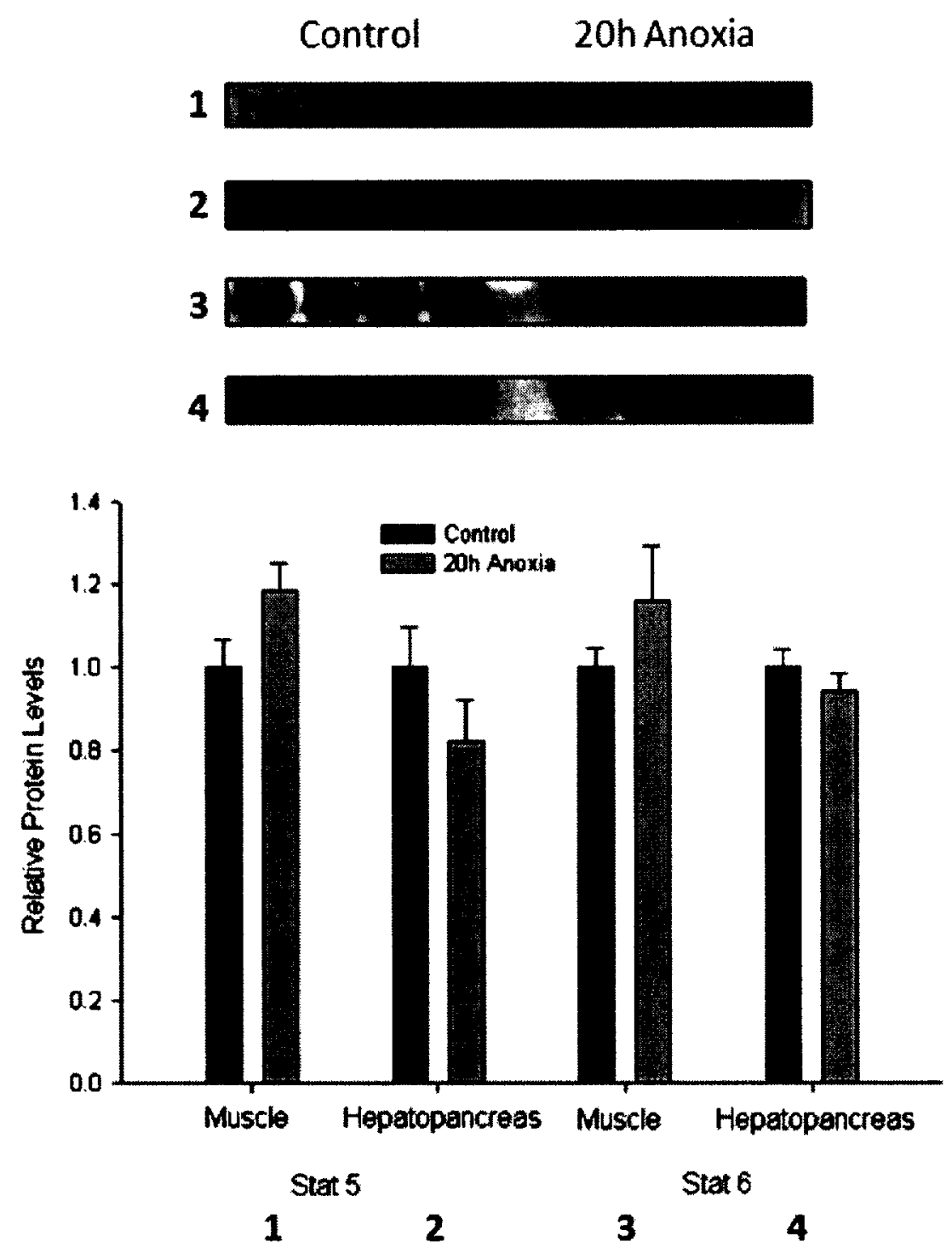

Figure 3.17: Effect of $20 \mathrm{~h}$ anoxic exposure on Stat 5 and 6 protein levels in $O$.virilis tissues.

Changes in the relative levels of Stat 5 and 6 proteins in crayfish tissues in response to anoxia, as determined by western immunoblotting.

Data are means \pm SEM, $n=4$ independent trials for muscle and hepatopancreas. * indicates significantly different from the corresponding control, $P<0.05$. 
Table 3.3 Chapter 3 data summary

\begin{tabular}{|l|c|c|c|c|c|c|c|c|c|c|}
\hline & \multicolumn{2}{|c|}{$\begin{array}{c}\text { Protein } \\
\text { (Total) }\end{array}$} & \multicolumn{3}{c|}{ Protein } & \multicolumn{2}{c|}{ mRNA } & \multicolumn{2}{c|}{ ELISA } \\
\hline & M & H & \multicolumn{2}{|c|}{ M } & \multicolumn{2}{|c|}{ H } & M & H & M & H \\
\hline & & & Cyto & Nuc & Cyto & Nuc \\
& & & $\cdot$ & $\cdot$ & & & & \\
\hline $\begin{array}{l}\text { Smad 1/5-p } \\
\text { (S463/465) }\end{array}$ & $\leftrightarrow$ & $\uparrow$ & & & & & & & & \\
\hline $\begin{array}{l}\text { Smad 2-p } \\
\text { (S465/467) }\end{array}$ & $\leftrightarrow$ & $\uparrow$ & $\downarrow$ & $\leftrightarrow$ & $\uparrow$ & $\leftrightarrow$ & & & & \\
\hline Smad 3 & $\uparrow$ & $\leftrightarrow$ & & & & & $\uparrow$ & $\leftrightarrow$ & $\leftrightarrow$ & $\uparrow$ \\
\hline Smad 4 & $\leftrightarrow$ & $\leftrightarrow$ & & & & & & & & \\
\hline STAT 1 & & & & & & & $\uparrow$ & $\leftrightarrow$ & & \\
\hline STAT 1-p (T701) & $\uparrow$ & $\uparrow$ & & $\downarrow$ & & $\downarrow$ & & & $\leftrightarrow$ & $\downarrow$ \\
\hline STAT 2-p (T690) & $\leftrightarrow$ & $\downarrow$ & & & & & & & & \\
\hline STAT 3-p (T705) & $\uparrow$ & $\leftrightarrow$ & & & & & & & & \\
\hline STAT 5 & $\leftrightarrow$ & $\leftrightarrow$ & & & & & & & & \\
\hline STAT 6 & $\leftrightarrow$ & $\leftrightarrow$ & & & & & & & & \\
\hline
\end{tabular}

Note: Changes indicate statistical significance $(P<0.05)$; Westem Blotting $(n \geq 4)$, RT-PCR $(n \geq 4)$, ELISA $(n=4)$

Arrows indicate changes or level profiles, in response to anoxia 


\section{Chapter 4 \\ Activation of the tumor suppressor protein, p53 in the anoxia resistant crayfish}




\subsection{Introduction}

Under many types of stress conditions an animal must make changes at the cellular level that will determine the fate of its cells. When stress conditions become prolonged, and energetics are compromised to the point where cells are no longer viable, the process of programmed cell death (apoptosis) is often signaled. Many stress-tolerant organisms use strategies such as entry into a hypometabolic state to greatly extend viability and largely prevent extensive apoptosis signaling. The importance of this is understood when, over a period of months during which the animal is living in a hypometabolic, energy-restricted state, modifications must be made as to which cells, tissues and organs must be maintained for whole organism survival, and which can be sacrificed if necessary. Hence, looking at cell death signaling in a model of metabolic rate depression can provide clues not only to how survival is achieved under energy limited conditions, but also to tissue specific responses in the animal.

Activation of the tumor suppressor p53:

Among the key regulators of apoptosis and lifespan regulation is the stress activated transcription factor p53. It is called a tumor suppressor protein because of its role, initially discovered in cell cycle control, in apoptosis signaling in cancer models. P53 responds primarily to DNA damage, but is also known to respond to reduced oxygen and nutrients levels; however, the focus here will be on the DNA damage pathway of p53 activation. Upon DNA damage, multiple stress response signaling cascades are initiated, among them members of the phosphatidylinositol 3-kinase superfamily (some of which have been discussed in previous chapters) including the ataxia telangiectasia mutated (ATM) and ATM-and Rad3 related (ATR) kinase (Yang et al., 2003). Working upstream 
of $\mathrm{p} 53$, both ATM and ATR kinases can be activated by DNA damage, reduced oxygen levels (hypoxia) and reoxygenation (FIG 4.1) (Hammond et al., 2003). From here ATM and ATR can directly phosphorylate p53, or indirectly through a cell cycle related checkpoint kinase (CHK2) (Yang et al., 2003). Control of $p 53$ :

Phosphorylation control of $\mathrm{p} 53$ is crucial to regulating its activity. In an unstressed state, there is little apoptosis above a basal level of expression, and so there is often very little p53 transcriptional activity. Under these conditions, $\mathrm{p} 53$ is typically bound to the mouse double minute 2 (MDM2) protein, an E3 ubiquitin ligase that promotes proteasome mediated degradation of $\mathrm{p} 53$ (Lavin and Gueven, 2006). Phosphorylation of either 553 or MDM2 releases $\mathrm{p} 53$ from the complex, but acetylation, sumoylation and methylation may also modify the pair (Lavin and Gueven, 2006). Phosphorylation of p 53 can also be used to block ubiquitination sites, increasing the stability of the transcription factor (Lavin and Gueven, 2006). In terms of its transcriptional activity, the C-terminus of $\mathrm{p} 53$ is the main target of modification. Through phosphorylation or acetylation of the C-terminus, or through recruitment of co-factors (ex. p300/CBP), that in turn promote modification of the C-terminus, ubiquitination can largely be prevented, conferring greater p 53 stability (Lavin and Gueven, 2006). In $C$. elegans, the p53 homolog (CEP-1), is suppressed via deacetylation and as a result the nematode experiences increased lifespan (see Appendix B.5) P53, apoptosis and the cell cycle:

Apoptosis, despite its connotations, is not an uncommon event. Typically, there is a constitutive basal level of apoptosis used as a quality control mechanism. Programmed 
cellular death, in a stress-induced mechanism, however, is characterized by chromatin damage and subsequent DNA damage (Ferraro and Cecconi, 2007). DNA damage signals are mediated through $\mathrm{p} 53$, which initiates apoptosis via two primary means; the caspase signaling cascade and prolonged attenuation of the cell cycle. The caspases are a conserved family of proteases, which trigger (among other responses) mitochondrial pore formation, formation of the apoptosome and activation of further caspase family proteases in the manner of an amplified signaling cascade (Alenzi et al., 2010). The second mechanism is through cell cycle attenuation. When DNA damage is not induced by genotoxic stress, p53 can be used to attenuate the cell cycle over a short period, which acts as a correctional mechanism in cell division (Lavin and Gueven, 2006). However, when activated in response to strong or prolonged stress signals, p53 is able to attenuate the cell cycle to the point of promoting cellular death. By targeting the cell cycle inhibitor p21, complexes between cyclins and cyclin dependent kinases (CDKs) (specifically cyclin E-CDK2) are unable to act as cell cycle progression kinases, and the cell cycle stalls at the $G_{1} / S$ phase barrier (Lavin and Gueven, 2006).

Anoxia and p53 signaling in crayfish

In this chapter, the response of p53 and multiple other signaling elements that respond to DNA damage were analyzed with respect to anoxia stress in crayfish tail muscle and hepatopancreas. ATM and ATR kinase levels were measured, followed by p53 protein levels and an assessment of p53 phosphorylation at the sites known to be phosphorylated by ATM and ATR kinase (Serine 15 and 37) in response to DNA damage or reduced oxygen signaling. The capacity for DNA binding by phospho-p53 was also measured, to confirm that nuclear activity manifested in transactivation of gene targets. 
This was followed by transcript analysis of a potentially pro-apoptotic downstream target the etoposide induced (ei24) gene. Following this, both inhibitor (MDM2) and activator (p19 ARF) protein levels in response to low oxygen stress, were measured. Contrary to what may be expected of $\mathrm{p} 53$ in a stress responsive animal, a distinct profile of $\mathrm{p} 53$ activation was seen in both crayfish tissues. Where the two tissues differed in their p53 regulation profiles was the nuclear presence of phopho-p53. Hepatopancreas showed no increase in nuclear presence, and decreased DNA binding, potentially indicating a protective response, whereas tail muscle showed the opposite trend. Despite this, the activity profile of p53 may be suggestive of a surprising, protective role in crayfish defense against low oxygen stress.

\subsection{Materials and Methods \\ 4.2.1. Animals}

Crayfish were treated and tissue samples were collected as described in Chapter 2.

\subsubsection{RNA extraction, $c D N A$ synthesis, primer design and $R T-P C R$}

Total RNA was isolated from crayfish tissues and cDNA was reverse transcribed and serially diluted as described in Chapter 2. Forward and reverse primers for ei24 were designed from conserved regions in these genes based on DNA alignment of the sequences from both vertebrate and invertebrate species. Following initial work using consensus primers, perfect (crayfish-specific) primers were then designed from crayfish sequences and used for quantification of transcript levels. Primers for the control gene, $\alpha$ tubulin, were as in Chapter 2. Primers and cDNA product sizes are listed in Table 4.1.

PCR reactions were composed as described in Chapter 2. The PCR program was as follows: $7 \mathrm{~min}$ at $94^{\circ} \mathrm{C}$ followed by 35 cycles of $1 \mathrm{~min}$ at $94^{\circ} \mathrm{C}, 1 \mathrm{~min}$ at a predetermined annealing temperature $\left(58^{\circ} \mathrm{C}\right)$, and $1.5 \mathrm{~min}$ at $72^{\circ} \mathrm{C}$. The final step was 
$72^{\circ} \mathrm{C}$ for $10 \mathrm{~min}$. PCR products were separated on a $1 \%$ agarose gel stained with ethidium bromide, visualized using the ChemiGenius imaging system (Syngene, Frederick, MD, USA) under UV light and quantified using the GeneTools program. The bands from the most dilute cDNA sample that gave visible product were used for quantification to ensure that the products had not reached amplification saturation. PCR products were sequenced by DNA Landmarks (St.-Jean-sur-Richelieu, QC) and sequences were verified as encoding the correct genes using the program BLASTN (http://www.ncbi.nlm.nih.gov/blast) at the NIH.

\subsubsection{Protein extracts and western blotting}

Protein extracts were prepared from tissue samples of control and $20 \mathrm{~h}$ anoxiaexposed crayfish. Equal amounts of protein $(20-30 \mu \mathrm{g})$ were loaded into each lane of $10 \%$ SDS polyacrylamide gels, electrophoresed and then electroblotted onto polyvinylidene difluoride (PVDF) membranes as described in Chapter 2. After the transfer of proteins, membranes were blocked for $15 \mathrm{~min}$ in Tris buffered saline containing Tween-20 (TBST: $20 \mathrm{mM}$ Tris base, $140 \mathrm{mM} \mathrm{NaCl}, 0.1 \% \mathrm{v} / \mathrm{v}$ Tween-20) with $2.5 \%$ non-fat dried milk added. Blots were probed overnight with primary antibodies (listed in TABLE 4.2). All primary antibodies were diluted 1:1000 in TBST. After incubation, blots were washed several times with TBST and then incubated at RT for $1.5 \mathrm{~h}$ with HRP-linked goat anti-rabbit IgG (Cell Signaling, diluted 1:2000 in TBST). Immunoblots were developed using enhanced chemiluminescence.

\subsubsection{Preparation of nuclear extracts and DNA- binding assay}

Nuclear extracts for DNA-binding assay were prepared as described in chapter 2 . Aliquots containing equal amounts of protein from each sample $(20 \mu \mathrm{g} / \mathrm{well})$ were then 
used to assess the amount of binding by p 53 to its DNA binding element as described in Chapter 2. The sequence of the biotin-conjugated probe was 5'- BiotinTACCCGGGCATGTCTAAGCATGCTG-3', whereas the complementary sequence was 5'- CAGCATGCTTAGACATGCCCGGGTA- 3'. Aliquots of nuclear extracts containing $20 \mu \mathrm{g}$ of protein were combined with $50 \mu \mathrm{L}$ of $1 \mathrm{x}$ protein binding buffer [ $10 \mathrm{mM}$ Hepes, pH 7.9, $50 \mathrm{mM} \mathrm{KCl}, 0.5 \mathrm{mM}$ EDTA, $3 \mathrm{mM} \mathrm{MgCl} 2,10 \%$ v/v glycerol, $0.5 \mathrm{mg} / \mathrm{mL}$ BSA, $0.05 \% \mathrm{NP}-40,1 \mu \mathrm{g}$ salmon sperm DNA (BioShop), $0.5 \mathrm{mM} \mathrm{DTT}$, and $\mathrm{NaCl}$ (variable amount was used based on the volume of nuclear extract added, final concentration was always $40 \mathrm{mM}$ )]. The plate was incubated at RT with mild agitation for $75 \mathrm{~min}$ and then washed 4 times with wash buffer. Aliquots of $60 \mu \mathrm{L}$ of the primary antibody for $\mathrm{p} 53-\mathrm{p}$ (s15) (Cell Signaling) were diluted 1:250 in PBS, then added to the wells. The plate was incubated for $3 \mathrm{~h}$. The remainder of the procedure was as described in Chapter 2 .

\subsubsection{Electromobility Shift Assay}

In order to confirm probe efficiency for the DNA-binding activity ELISA, an electromobility shift assay was performed to visualize probe binding. First, biotinylated probe from the DNA binding activity assay $(250 \mathrm{pmol} / \mu \mathrm{l})$ was diluted to $10 \mathrm{pmol} / \mu \mathrm{l}$. Aliquots of $4 \mu \mathrm{l}(8 \mu \mathrm{g})$ of nuclear extract were mixed with $1 \mu \mathrm{l}$ DEPC treated $\mathrm{H}_{2} \mathrm{O}$ and 4 $\mu$ EM SA master mix; 5X EMSA binding buffer contained [50mM Tris $\mathrm{HCl}, \mathrm{pH} 7.8$, $250 \mathrm{mM} \mathrm{NaCl}, 5 \mathrm{mM}$ EDTA and $25 \%$ glycerol combined with $1 \mu \mathrm{g} / \mu \mathrm{l}$ Poly d(I-C) in a 1:1 v:v ratio. Samples were vortexed and incubated at RT for $5 \mathrm{~min}$ and then $1 \mu \mathrm{lTF}$ probe was added and the mixture was vortexed again before placing in the thermocycler for $30 \mathrm{~min}$ at $15^{\circ} \mathrm{C}$. The positive control was biotinylated E2F Probe (Panomics EMSA Kit 7104) tested with turtle (Trachemys scripta elegans) nuclear extract, in the same mix 
as above. Negative controls for no protein and no probe were also run, with adjustments to water volume as needed to keep total volume at $9 \mu$.

Following incubation, samples were mixed with $1 \mu \mathrm{l}$ 6x DNA Loading dye (BioShop) prior to loading on a $6 \%$ non-denaturing polyacrylamide gel [ $1 \mathrm{ml} 10 \mathrm{X}$ TBE (chilled), $4 \mathrm{ml} 30 \%$ bis-acrylamide, $625 \mu 180 \%$ glycerol, $14.375 \mathrm{ml}$ deionized sterile $\mathrm{H}_{2} \mathrm{O}$, $300 \mu 110 \%$ APS and $20 \mu \mathrm{l} \mathrm{TEMED} \mathrm{-} \mathrm{total} 2 \times 10 \mathrm{ml} / \mathrm{gel})$. The gels were cast as in the Western blotting procedure (above). Gels were pre- run in chilled $0.5 \mathrm{x}$ TBE for $10 \mathrm{~min}$ at $120 \mathrm{~V}$. Samples were then loaded into the gels and run at $120 \mathrm{~V}$ for $70 \mathrm{~min}$ at $4^{\circ} \mathrm{C}$ (fridge). Following electrophoresis, proteins were transferred to Pall Biodyne B nylon membranes. Membranes were pre-soaked in $0.5 \mathrm{x}$ TBE and then gels were sandwiched to membranes as described for the Western blotting protocol. Proteins were transferred in $0.5 \times$ TBE (chilled) for $30 \mathrm{~min}$ at $300 \mathrm{~mA}$ at $4^{\circ} \mathrm{C}$. Once transfer was complete, membranes were stored between dry filter paper, and the oligos were fixed to membranes in an oven $\left(80^{\circ} \mathrm{C}\right)$ for $1 \mathrm{~h}$. Following this, membranes were washed on a rocking platform with $5 \mathrm{ml}$ (per membrane) of 1x EMSA Blocking Buffer (diluted from Panomics CS7535) for 30 min at RT. The blocking buffer was decanted and to it $5 \mu \mathrm{l} / \mathrm{ml}$ Streptavidin-HRP mixture (BioShop) was added. This mixture was reapplied to the membrane and left to incubate on a rocking platform for a further $30 \mathrm{~min}$ at RT. Finally, membranes were washed 3 times, consecutively, with $1 \mathrm{x}$ wash buffer (diluted from Panomics 7537). The membranes were then exposed using ECL solutions as in the Western blotting protocol.

\subsubsection{Data Analysis}

All data were analyzed as described in Chapter 2. 
Table 4.1 List of Primers used in Chapter 4

\begin{tabular}{|c|c|c|c|}
\hline Gene/primer & Primer sequence (5'-3') & $\begin{array}{c}\text { Annealing } \\
\text { temp. }\left({ }^{\circ} \mathbf{C}\right)\end{array}$ & $\begin{array}{c}\text { Expected } \\
\text { product size } \\
\text { (bp) }\end{array}$ \\
\hline ei24 perfect forward & TATAGCATGTGCACTGTGCC & \multirow{2}{*}{58} & $\sim 220$ \\
\hline ei24 perfect reverse & GTCTGTACTCCGGGAACAAC & 58 & $\sim$ \\
\hline
\end{tabular}

Table 4.2 List of primary antibodies used in Chapter 4

\begin{tabular}{|l|l|l|}
\hline \multicolumn{1}{|c|}{$\begin{array}{c}\text { Primary } \\
\text { Antibody }\end{array}$} & \multicolumn{1}{|c|}{ Company } & \multicolumn{1}{c|}{ Catalogue \# } \\
\hline$P 53$ & Cell Signaling (Danvers, MA, USA) & $\# 2524$ \\
\hline$P 53-p(S 15)$ & Cell Signaling (Danvers, MA, USA) & $\# 9284$ \\
\hline$P 53-p(S 37)$ & Cell Signaling (Danvers, MA, USA) & $\# 9289$ \\
\hline$M D M 2$ & $\begin{array}{l}\text { Santa Cruz biotechnology (Santa Cruz, CA, } \\
\text { USA) }\end{array}$ & sc-7918 \\
\hline$P 19-A R F$ & Cell Signaling (Danvers, MA, USA) & $\# 2407$ \\
\hline$A T M$ & Genscript (Piscataway, NJ, USA) & $\mathrm{A01222}$ \\
\hline$A T R$ & Genscript (Piscataway, NJ, USA) & $\mathrm{A01253}$ \\
\hline
\end{tabular}




\subsection{Results}

4.3.1. Protein expression of ATM and ATR in response to anoxia

Changes in the protein levels of ATM and ATR in response to anoxia were assessed using immunoblotting (FIG.4.2). The polyclonal ATM and ATR antibodies cross-reacted with protein bands of $\sim 350$ and $300 \mathrm{kDa}$, respectively, which are their expected sizes. ATM protein levels increased in both tissues during anoxia, by $1.3 \pm 0.03$ fold in tail muscle, and $3.1 \pm 0.4$ fold in hepatopancreas (both $P<0.05$ ). ATR followed this pattern by showing increased protein levels in both tissues as well. ATR levels in tail muscle exhibited a $1.5 \pm 0.2$ fold increase after $20 \mathrm{~h}$ anoxia exposure, whereas hepatopancreas ATR levels rose $2.4 \pm 0.2$ fold (both $\mathrm{P}<0.05$ ) over control values.

\subsubsection{Protein expression of $p 53$ in response to anoxia}

Protein levels of p53 (in response to anoxia) were assessed using immunoblotting (FIG.4.3). The polyclonal anti-p 53 antibodies cross-reacted with a protein band of $\sim 53$ $\mathrm{kDa}$, which is its expected size. Total relative protein levels of $\mathrm{p} 53$ were unchanged in tail muscle (left) and hepatopancreas (right) during anoxia.

\subsubsection{Total protein expression of $p 53-p(s 15)$, and cellular localization in response to anoxia}

To become stable, and free from binding with the p53 inhibiting ubiquitin ligase, MDM2, p53 may be phosphorylated. Upon phosphorylation, p53 can migrate to the nucleus to express its transcriptional activities. Changes in relative amounts of phosphorylated p53 (phosphorylated at serine 15) in response to anoxia were assessed using immunoblotting (FIG.4.4). The polyclonal anti-p53-p (Ser 15) antibodies crossreacted with a protein band at $\sim 53 \mathrm{kDa}$, which is its expected size. Phosphorylation profiles in 'total' protein extracts show that in both tissues there was a significant 
increase in phosphorylation at serine 15 under anoxia. In tail muscle phosphoprotein content increased by $1.8 \pm 0.2$ fold, and in the hepatopancreas the increase was $2.0 \pm 0.3$ fold during anoxia (both $\mathrm{P}<0.05$ ). Phosphorylation level changes with respect to cellular localization, either in the cytoplasmic fraction or nuclear fraction, were also assessed by immunoblotting (FIG. 4.5). In tail muscle, both cytoplasmic and nuclear fractions showed significant increases in phosphorylated Ser 15 content under anoxia; levels rose by $1.5 \pm 0.1$ fold and $1.4 \pm 0.1$ fold, respectively (both $P<0.05$ ). However, in the hepatopancreas under anoxia, increases in Ser 15 phosphorylation were seen only in the cytoplasmic fraction $(2.2 \pm 0.1$ fold; $P<0.05)$, whereas levels in the nuclear fractions remained constant.

\subsubsection{DNA binding activity of $p 53$ in response to anoxia}

Changes in the DNA-binding activity of nuclear p53 (phosphorylated at serine 15) in response to anoxia were assessed using a transcription factor binding assay (FIG.4.6). The relative amount of DNA binding in tail muscle extracts increased significantly (by $2.2 \pm 0.3$ fold; $P<0.05$ ) under anoxia. Conversely, there was a significant decrease in DNA binding activity by p 53 in hepatopancreas extracts; binding was reduced by $60 \pm 10$ $\% ; \mathrm{P}<0.05)$. Probe specificity was confirmed through EMSA banding.

\subsection{5. cDNA cloning of crayfish ei24}

A PCR product of 224 bp was from total RNA prepared from crayfish tail muscle and hepatopancreas using the crayfish-specific ei24 primers (originally designed from the consensus sequences of vertebrate and invertebrate species and then adjusted based on the initial cDNA sequence retrieved). The product was analyzed by BLAST, and did not appear to conform to other ei24 sequences. The non-specific identity of the product is in 
keeping with low homologies for other crustacean gene products (there are very limited amounts of data about crustacean sequences in gene banks), and as such will be identified as an ei24-like protein. FIG.4.7A shows the partial nucleotide sequence with the corresponding amino acid sequence. The partial crayfish amino acid sequence was 73 amino acids. FIG. 4.7B shows the homology between the isolated cDNA from $O$. virilis and the comparable fragment from the water flea. FIG.4.7C shows a homology tree produced from the alignment of the partial $O$. virilis ei24-like cDNA sequence with ei24 from fruit fly (Drosophila melanogaster) and water flea (Daphnia pulex). The homology tree shows the separation of sequence homology, within the invertebrate arthropods, there is $42 \%$ similarity, which increases to $47 \%$ similarity between the crustaceans.

\subsubsection{Transcript expression of ei24-like protein in response to anoxia}

ei24 is a p53 inducible gene, linked with potential apoptosis signaling. Changes in the relative mRNA transcript levels of ei24-like protein in response to $20 \mathrm{~h}$ of anoxia were assessed using RT-PCR and perfect primers designed from the crayfish sequence (FIG.4.8). Representative RT-PCR bands of ei24 amplicons (shown below the histogram) are shown in conjunction with the graphical presentation of mean values. ei24 transcript levels were normalized with tubulin bands amplified from the same tissue samples. While ei24 levels remained constant in the hepatopancreas under anoxia, transcript levels in tail muscle rose significantly $(1.8 \pm 0.1$ fold; $\mathrm{P}<0.05)$.

\subsubsection{Protein expression of $p 53-p$ (S37) in response to anoxia}

The $\mathrm{p} 53$ protein is also phosphorylated on serine 37 by the upstream ATM/ATR kinases. The polyclonal anti-p53-p (S37) antibodies cross-reacted with a protein band of $\sim 53 \mathrm{kDa}$ in crayfish extracts, which is the expected size for p53. Like the phospho-Ser 
15 response, the relative amount of phospho-Ser 37 also increased in both tail muscle and hepatopancreas during anoxia; levels were significantly increased by $1.7 \pm 0.1$ and $1.4 \pm$ 0.06 fold, respectively $(P<0.05)$ (FIG.4.9).

\subsubsection{Protein expression of $M D M 2$ in response to anoxia}

Changes in the protein levels of MDM2 in response to anoxia are shown in

FIG.4.10. The polyclonal MDM2 antibodies cross-reacted with a protein band of $\sim 90$ $\mathrm{kDa}$, which is its expected size. In both tissues, MDM2 protein levels decreased significantly in response to anoxia. In tail muscle MDM2 content dropped by $40 \pm 6 \%$; hepatopancreas showed a $40 \pm 10 \%$ decrease (both $\mathrm{P}<0.05$ ).

\subsubsection{Protein expression of $p 19 A R F$ in response to anoxia}

Changes in the protein levels of p19 ARF in response to anoxia are shown in FIG.4.11. The polyclonal p19 ARF antibodies cross-reacted with a protein band of $\sim 19$ $\mathrm{kDa}$, which is the expected size. In both tissues, p19 ARF protein levels increased significantly in response to anoxia. In the tail muscle protein levels increased by $1.2 \pm$ 0.03 fold, and in hepatopancreas levels rose by $1.6 \pm 0.1$ fold (both $P<0.05$ ), in comparison to aerobic controls.

\subsection{Discussion}

Programmed cellular death is an innate and highly important function. Its regulation is critical, especially in the context of cellular proliferation rates. To that extent, the tumor suppressor protein, $\mathrm{p} 53$ has become one of the foremost cancer research targets because of its role in apoptosis signaling.

By studying models of hypometabolism or metabolic adaptation, like the anoxia resistant crayfish, we are not only able to see how cells are able to circumvent apoptosis 
to sustain long term viability under stressful conditions, but we are able to see specifically how p53 is regulated in response to oxygen deprivation. While there may not seem to be a clinical crossover between a crayfish model and human disease states, in fact there is a significant link between oxygen limitation and the malignancy of tumors. Hypoxic conditions, which are typically oxygen levels under $20 \%$ (ranging to $1 \% \mathrm{O}_{2}$ ), are common in most tumor types, resulting from rapid oxygen consumption due to excessive cellular proliferation (Hammond and Giaccia, 2005). Tumor cells survive prolonged hypoxic conditions, by lowering their metabolic rates in order to compensate for the lack of developed vasculature. As tumor cells proliferate rapidly, often they cannot be vascularized at the same rate which results in lowered blood delivery, and subsequent reductions in nutrients and oxygen, and as well as removal of cellular waste products. When vasculature is developed and blood flow is resumed, reoxygenation creates a significant amount of oxidative stress. The electron transport chain, overwhelmed at the influx of oxygen produces reactive oxygen species (ROSs), including superoxides $\left(\mathrm{O}_{2}{ }^{-}\right)$, hydroxyl-radicals $(\mathrm{OH})$ and hydrogen peroxide $\left(\mathrm{H}_{2} \mathrm{O}_{2}\right)$. In stress adapted animals this influx of ROSs may be mediated by a range of antioxidant proteins up-regulated during the hypometabolic period (Hermes-Lima and Zenteno-Savin, 2002), but in tumors, in which antioxidant genes are frequently suppressed, reoxygenation can lead to substantial DNA damage (Hammond and Giaccia, 2005). Hence, an activation of p53 leading to triggering of apoptosis, would become a major factor in tumor suppression, under oxygen limitation. However, loss of p53 expression is common in many tumors, thereby preventing apoptosis and allowing tumor survival. Indeed, finding ways to target p53 (re-) activation in tumor cells has become a major therapeutic task, 
and is the subject of many reviews (including; Amaral et al., 2010; Qian and Chen, 2010; Meek, 2009).

Contrary to what would be expected (and indeed in opposition to the general concept of this chapters hyposthesis), in a state where ectopic apoptosis would be severely deleterious to the cell, the freshwater crayfish $O$. virilis showed what could be classified as a pro-p53 response to anoxia (Table 4.3), and yet, given its ready survival of a $20 \mathrm{~h}$ anoxia exposure, any significant level of apoptosis seems unlikely. Hence, the regulation of p53 as part of crayfish anoxia resistance may point to an alternate mechanism of p53 control, one in which, despite high levels of p53 expression, cells remain viable.

ATM and ATR kinase signaling in response to anoxia resistance

Though the mechanism is not fully elucidated, the ATM and ATR kinases are able to respond to DNA breaks. They typically respond to different stimuli; double strand breaks (via IR) trigger ATM whereas UV/stalled replication forks trigger ATR (Yang et al, 2003). However, both kinases are also activated by other types of signal; for example, ATM and ATR show an interesting response to hypoxia and re-oxygenation. Activated by hypoxia, ATR (but not ATM) phosphorylates p53 at serine 15, with ATM suspected to play less of a role because of a lack of DNA damage under hypoxia (Hammond et al., 2003). However, under anoxic conditions in $O$. virilis, the protein levels of both ATM and ATR kinases increased significantly in both tissues with respect to control levels. While this conforms with the oxygen sensitive nature of ATR, it may also suggest that under prolonged oxygen deprivation, ATM could also be induced into greater protein expression. ATM, in the tumor environment, is typically signaled only by high levels of 
DNA damage, and is subsequently seen to be more responsive during the reoxygenation period in which significant generation of ROSs have such an effect (Hammond et al., 2003).

\section{P53 activity and regulation under anoxia}

Initially, 553 protein levels were analyzed, and relative protein expression remained unchanged in both tissues under anoxia. On the other hand, analysis of p53 phosphorylation did indicate an interesting event. In conjunction with the increased protein expression of ATM and ATR kinases, enhanced phosphorylation of p53 occurred during anoxia. Again, it is worth reiterating that under hypoxic conditions, it has been observed that there are no significant levels of DNA damage to signal the ATR/ATM kinases (Hammond et al., 2003), and even under anoxia, it remains unlikely that DNA damage is responsible for $\mathrm{p} 53$ signaling. What we may be seeing is an activation of $\mathrm{p} 53$ in response to a stalling of DNA replication, or even an accumulation of quality control signals with respect to DNA synthesis, which cause ATR (already known to respond to hypoxia) and ATM (known to primarily respond to re-oxygenation) to phosphorylate p53 at serine 15 and 37 . Hence, the activation profile (Table 4.3), along with going against a general anti-apoptotic hypothesis also contravenes the lifespan extension model in $C$. elegans, whereby p53 is deacetylated (see Appendix B.5), and inactivated.

Phosphorylation of both serine 15 and serine 37, sites known to be primary targets of the ATM/ATR kinases, significantly increased in 'total' protein extracts of both crayfish tissues under anoxia. The phospho-form that is widely acknowledged as the marker for the DNA damage response is Ser 15 . In both tail muscle and hepatopancreas cytoplasmic fractions, the relative levels of phospho-p 53 Ser 15 increased, but only in tail 
muscle did nuclear levels also increase during anoxic exposure. Conversely, in the nuclear fraction of hepatopancreas, Ser 15 phosphorylation levels remained constant. DNA binding activity profiling (through ELISAs), showed that $\mathrm{p} 53$ binding to DNA increased in anoxic muscle, correlated with the increased nuclear levels of phosphorylated p53 during anoxia. In the hepatopancreas, however, a significant decrease in DNA binding was seen, compared to aerobic controls, indicating a potentially tissue specific mechanism of p53 regulation. This was further supported by transcript levels of the p53 inducible ei24 protein. Following both nuclear and phospho-profiling, ei24 transcript levels were seen to increase in the muscle, but not the hepatopancreas. Overexpression of this gene is linked to apoptosis, marked by inhibited cellular proliferation in cell colonies (Gu et al., 2000). Though its mechanism of activity is largely unknown, cellular death signaling linked to ei24 activity may be repressed through Bcl-xl (an antiapoptosis protein) activity (Gu et al., 2000). What is understood, however, is that activity of ei24 is not able to induce apoptosis alone, and it is likely a component in a much larger apoptotic signaling cascade (Gu et al., 2000). Hence while this result is insufficient evidence of induced apoptosis, it is a viable marker of p53 activity in the $O$. virilis tail muscle under anoxia.

Patterns of p53 expression and the roles of cytoplasmic vs. nuclear p53

Tail muscle and hepatopancreas showed parallel responses by $\mathrm{p} 53$ parameters to anoxia up to the nuclear level, diverging in terms of nuclear levels of phosphorylated p53 and DNA binding activity analyses. What is clear is that $\mathrm{p} 53$ exhibits significant activation under anoxia in $O$. virilis. While in the tail muscle, a pattern suggesting core p53 activity is observed, i.e. a capacity for transcriptional activity in response to anoxia, 
in the hepatopancreas, the newly activated $\mathrm{p} 53$ appears to be located solely in cytoplasm. The tissue specific location of these proteins is interesting since p53 is traditionally thought of as a primarily nuclear protein. Recently though, cytoplasmic roles of p53 have commanded more research interest, through suggested alternate means of apoptosis signaling (Green, 2009).

One of the major pro-apoptotic transcriptional roles of $\mathrm{p} 53$ is to transactivate targets that will compromise mitochondrial membrane integrity. Being one of the primary mechanisms to induce apoptosis, pore formation in the outer mitochondrial membrane (OMM) causes release of apoptosis stimulating messengers (such as cytochrome c), which leads rapidly to cellular death. Pore stability is maintained by the physical interaction between anti- and pro- apoptotic $\mathrm{B}-\mathrm{cell}$ lymphoma $(\mathrm{Bcl})$ family proteins. The pro-apoptotic members of this family, when not bound by their anti-apoptotic counterparts, homodimerize and migrate to the OMM to initiate pore formation (Smith et al., 2008). Traditionally, through transactivation of pro-apoptosis Bcl family members (ex. Bax, PUMA, NOXA) and repression of the anti-apoptotic Bcl proteins (ex. Bcl-2, Bcl-xl), p53 pushes the balance of OMM stability to favour pore formation (Speidel, 2010). It is also able to transactivate caspase 8 , a member of the highly conserved proapoptotic protease family, which also affects the balance of pro- and anti- apoptotic $\mathrm{Bcl}$ proteins, pushing the cell towards apoptosis in conjunction with pro-apoptotic factor, Bid (which in turn increases Bax activity) (Speidel, 2010). In a cytoplasmic role, it has been demonstrated that $\mathrm{p} 53$, which is able to bind to the Bcl 2 homology (BH) domains of Bcl2 family proteins, can physically interact with Bak, Bax and Bad (all pro-apoptosis) in the cytoplasm, and consequently activate them. However, this cytoplasmic action alone is not 
strong enough to elicit apoptosis (Speidel, 2010). On the other side of the Bcl-2 family, p53 is also thought to be able to interact with the anti-apoptotic members (Bcl-2 and Bclxl) through its DNA binding domain and subsequently interfere with their ability to complex with the pro-apoptotic members (Mihara et al., 2003); this leaves the proapoptotic Bcls free to travel to the mitochondrial membrane. Hence, a cytoplasmic p53mediated apoptosis could be a possibility, but it appears to be dependent on the strength of its cytoplasmic levels, which would determine the extent of its ability to significantly interact with both pro- and anti- apoptotic members of the Bcl-2 family. Along with this, it appears that cytoplasmic p 53 and nuclear p 53 activity are both signaled in the same way, and work in conjunction with each other to fully stimulate apoptosis (Ferecatu et al., 2009). Hence, while it is capable of pro-apoptotic signaling, increased active cytoplasmic p53, alone, would likely not be an indicator of alternate apoptosis signaling.

A dichotomy was seen in the hepatopancreas pattern of $\mathrm{p} 53$ activity - an increase in stable p53 was indicated but an accumulation only in the cytoplasmic fraction. Given the importance of hepatopancreas function in $O$. virilis, along with no signs of significant tissue damage in anoxia, there may be an implied response to export p53 from the nucleus in order to prevent potential pro-apoptosis transcriptional events. Alternate posttranslational modifications of the export site of p53 can cause accumulation of cytoplasmic p53, along with interactions with other transcription factors in the nucleus (i.e. FoxO3) and even mono-ubiquitination by MDM2, which occurs even at low levels of MDM2 expression (Speidel, 2010). This last option would fit the profile of reduced MDM2 expression that was also seen in the anoxic hepatopancreas. The E3 ubiquitin ligase, MDM2, is known to control both nuclear export and degradation (proteasome 
mediated) of p53, and while poly-ubiquitination and p53 degradation accord with high expression levels of MDM2, low levels of MDM2 are only able to confer monoubiquitination and nuclear expulsion ( $\mathrm{Li}$ et al., 2003).

Contrast this to the tail muscle, where increased levels of phosphorylated p53 in the cytoplasmic and nuclear fractions were seen, along with increased DNA binding; this appears to be a clear profile of active p53. With apoptosis an unlikely outcome, despite the relative lack of importance in muscle preservation (compared to hepatopancreas, heart and brain tissues), the endpoint of this p53 activity is uncertain. There is the possibility, however, of a fairly novel pro-survival role for $\mathrm{p} 53$ (and its activator p19 ARF) in signaling a cell rescue mechanism, autophagy, under stress conditions. This is particularly interesting because $\mathrm{p} 53$, as an apoptosis activator, is commonly thought to largely repress autophagy signals; the two processes being in opposition, and regulated accordingly. In many cases, loss of autophagy function results in tumorigenesis through excess ROS production and increased DNA damage response (coupled with defective apoptosis) (Karantza-Wadsworth et al., 2007). However, when tumor cells exist in low energy states, due to hypoxic conditions, autophagy is employed to recycle molecular building blocks, and preserve cellular ATP levels (Balaburski et al., 2010).

Autophagy activation by $\mathrm{p} 53$ is thought to occur through a number of mechanisms; through induction of the damage regulated autophagy modulator (DRAM) and through inhibition of the mammalian target of rapamycin (mTOR) kinase (a potent autophagy inhibitor). DRAM, a lysosomal protein involved in both apoptosis and autophagy, is seen to be induced by $\mathrm{p} 53$ as part of its tumor suppression capacity (Crighton et al., 2007). DRAM is inactivated in certain cancers, in conjunction with p53, 
which indicates a shared proliferation mechanism (Crighton et al., 2007). In terms of p53 capacity to affect TOR activity, one of the major proposed mechanisms for this is through p53 transactivated targets which have the capacity to inhibit mTOR. Subunits of AMPK ( $\beta 1$ and $\beta 2$ ), and the PTEN phosphatase, both of which alter the phosphorylation state of the $\mathrm{mTOR}$ upstream activator (TSC1/2) to prevent its activation, are $\mathrm{p} 53$ targets (Balaburski et al., 2010). Along with this, the $\mathrm{p} 53$ family member, $\mathrm{p} 73$ is a direct transcriptional regulator of autophagy genes and is also negatively regulated by mTOR. Hence, p53 mediated suppression of mTOR is thought to be able to enhance autophagy further, under stress conditions, by maintaining p73 activity (Rosenbluth et al., 2008).

In accordance with the general profile of $\mathrm{p} 53$ activation seen in $O$. virilis, protein levels of the p19 ARF tumor suppressor increased significantly in both tissues with anoxia. This correlated further with the decreases in MDM2 protein levels (also seen in both tissues, under anoxia). P19 ARF physically interacts with MDM2, inhibiting its ubiquitin ligase activity, and allows p 53 to become activated and move into the nucleus (Xirodimas et al., 2002). It is also suspected, although the mechanism has not been elucidated, that p19 ARF is able (like p53) to stimulate autophagy under stressed conditions (Balaburski et al., 2010).

\section{Conclusions}

Anoxia exposure triggered specific patterns of $p 53$ response in tissues of the freshwater crayfish (Table 4.3). Phosphorylation of p53 on sites regulated by the ATM and ATR kinases (Ser 15, Ser 37) increased under anoxia, in conjunction with reduced protein levels of the inhibitor MDM2, and increased protein levels of the activator p19 ARF. Where the two tissues diverged was in the nuclear response by p53. Both tissues 
showed increased phospho-p53 content in the cytoplasm, though it is thought that this localized form of p53 essentially has very little apoptotic capacity. In the tail muscle, however, increased content of phospho-p53 in nuclear fractions in conjunction with increased DNA binding capacity and increased ei24 transcription suggests an active level of $\mathrm{p} 53$. While the crayfish is able to avoid apoptosis, the activation pattern goes against the expected reduction, predicted for these data. Despite this, there is a possibility that p53 signaling under the stressed anoxic state could be functioning to activate the cell rescue procedure of autophagy; which would conform with $\mathrm{p} 53$ regulation resulting in a (potentially) novel survival process for anoxia resistance. 


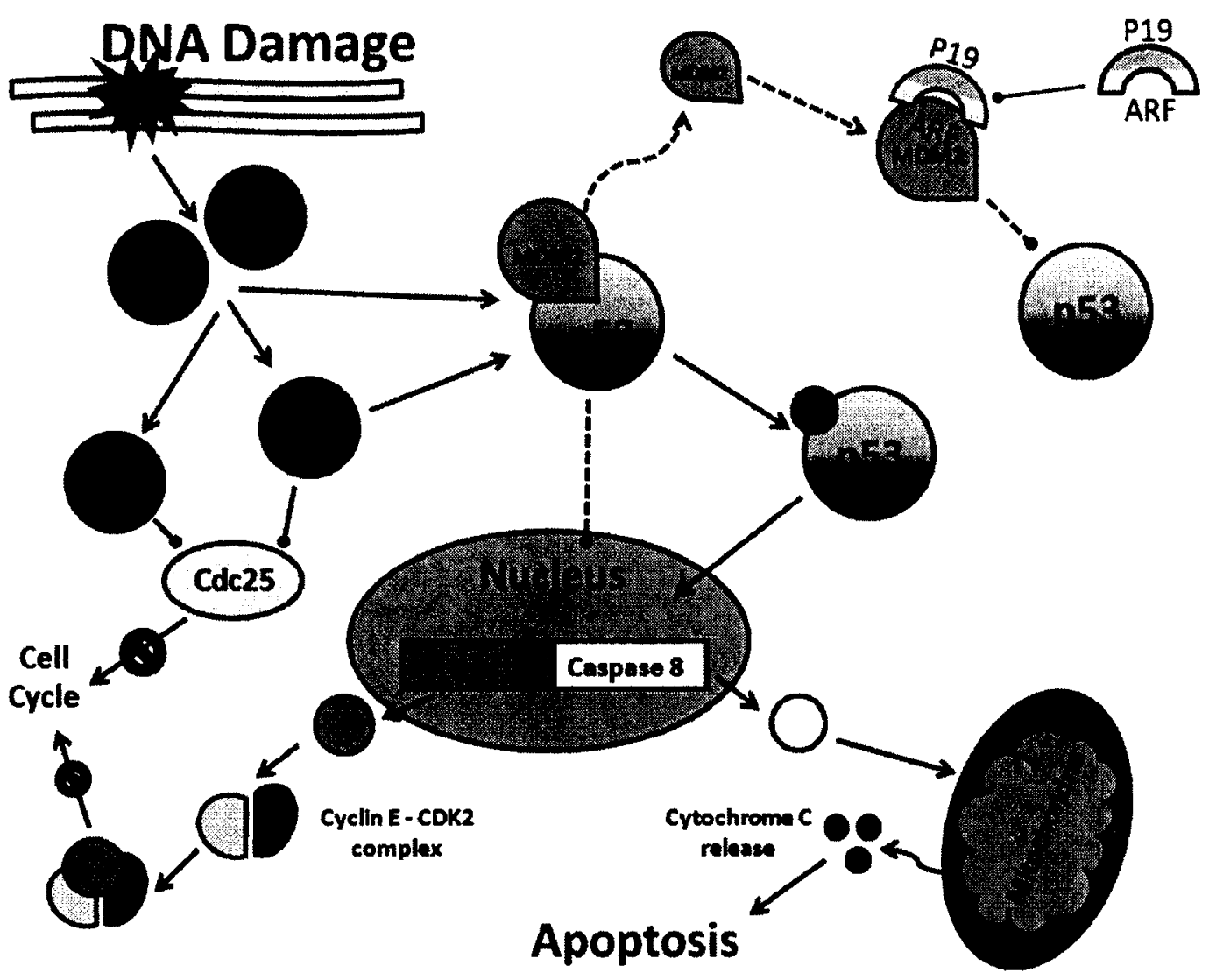

Figure 4.1: An overview of the p53 DNA damage response. The p53 tumor suppressor is signaled by a variety of stresses that result in DNA damage. Upstream of $\mathrm{p} 53$, the signaling kinases ATM and ATR mediate the stress response through the checkpoint kinases (CHK) and p53 directly. The MDM2 ubiquitin ligase, an inhibitor of p53 binds directly to the transcription factor, signaling its degradation. Phosphorylation of p53 or MDM2, causes a release, and allows p53 to enter the nucleus. Amongst transcriptional targets for p53 are the cell cycle inhibitor p21 and the protease caspase 8; transactivation of either could lead to cell death. Solid lines indicate canonical p53 signaling, dashed lines indicate anti-p53 activity. 
Control 20h Anoxia

1

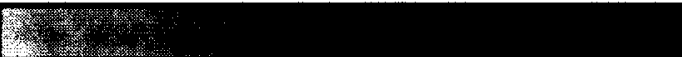

2

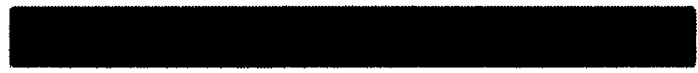

3

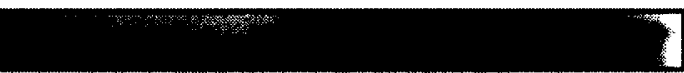

4
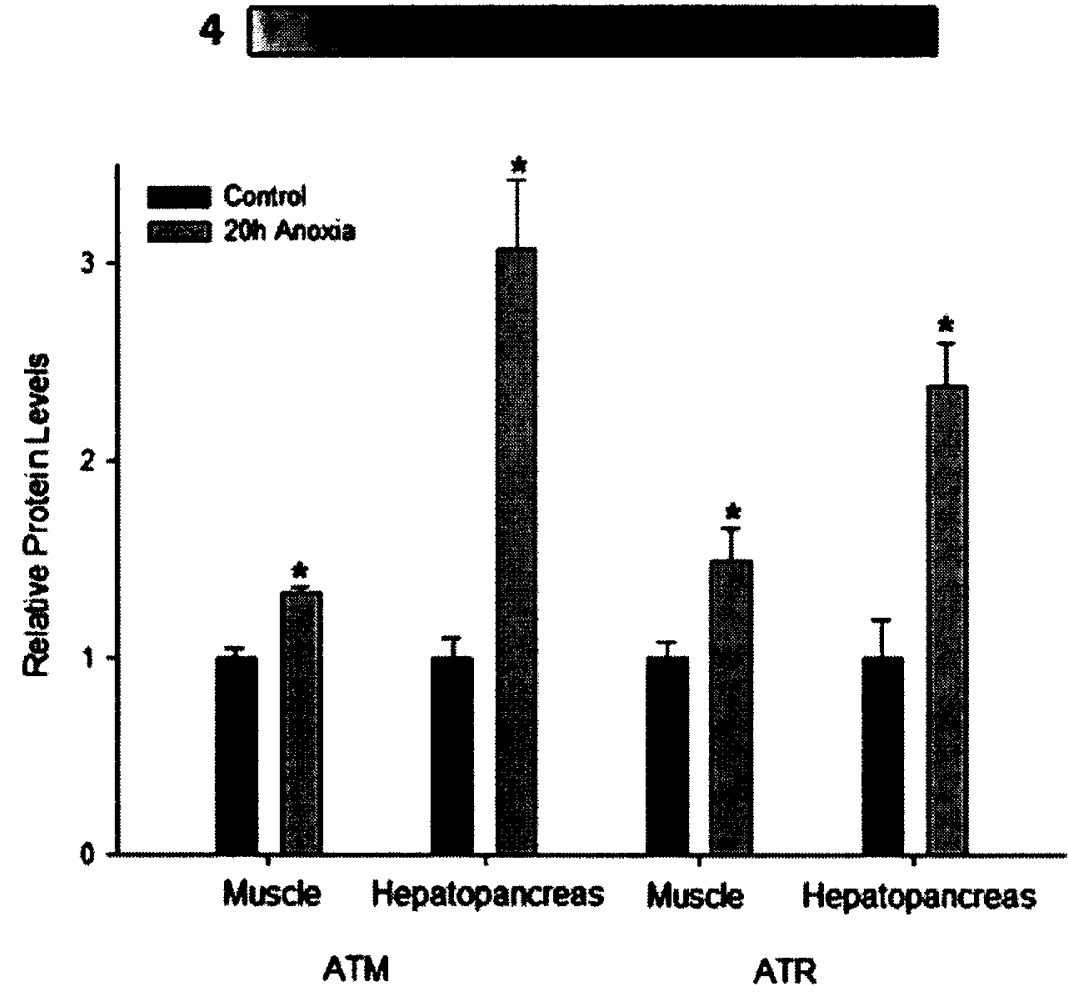

12

3

4

Figure 4.2: Effect of $20 \mathrm{~h}$ anoxic exposure on ATM and ATR activation in 0 . virilis tissues.

Changes in ATM and ATR protein levels in crayfish tissues in response to anoxia, as determined by western immunoblotting.

Data are means $\pm S E M, n=4$ independent trials for muscle and hepatopancreas. * indicates significantly different from the corresponding control, $P<0.05$. 


\section{Control 20h Anoxia}

1

2

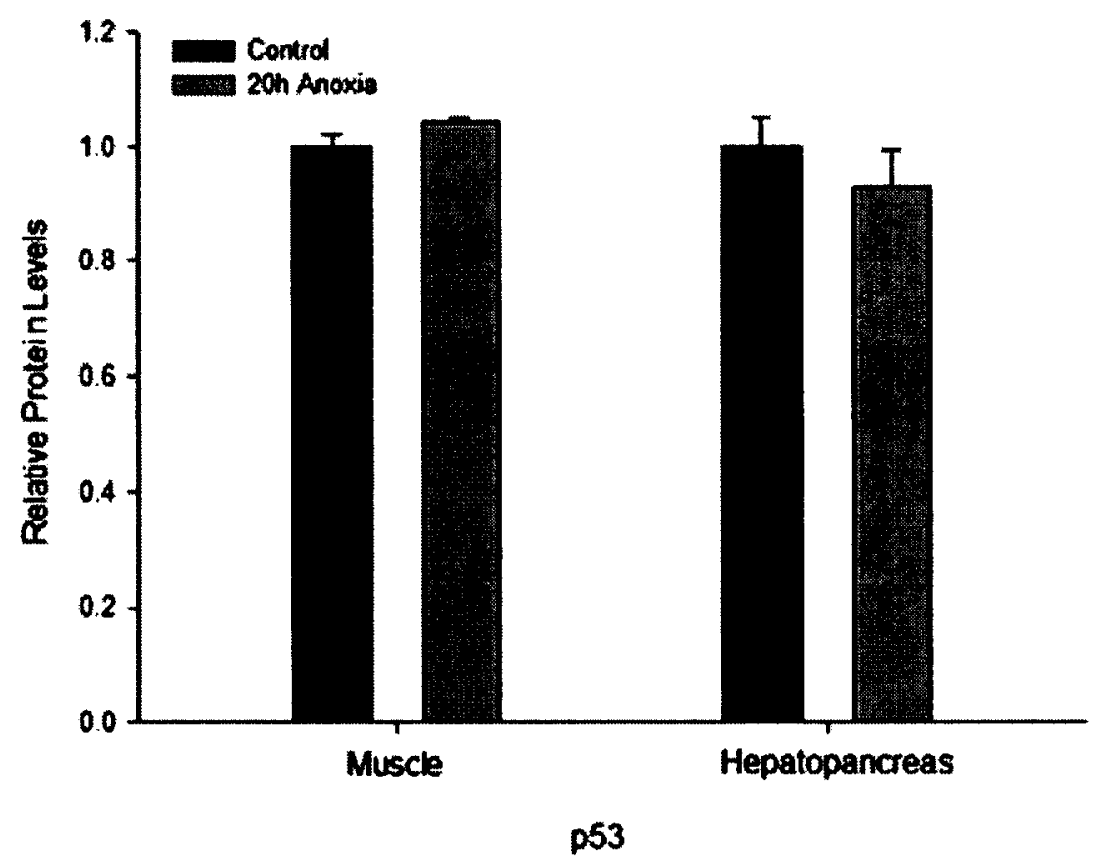

1

2

Figure 4.3: Effect of $20 \mathrm{~h}$ anoxic exposure on p53 protein levels in $O$. virilis tissues. Changes in p53 protein levels in crayfish tissues in response to anoxia, as determined by western immunoblotting.

Data are means \pm SEM, $n=4$ independent trials for muscle and hepatopancreas. 
Control 20h Anoxia

1

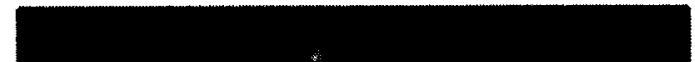

2

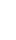
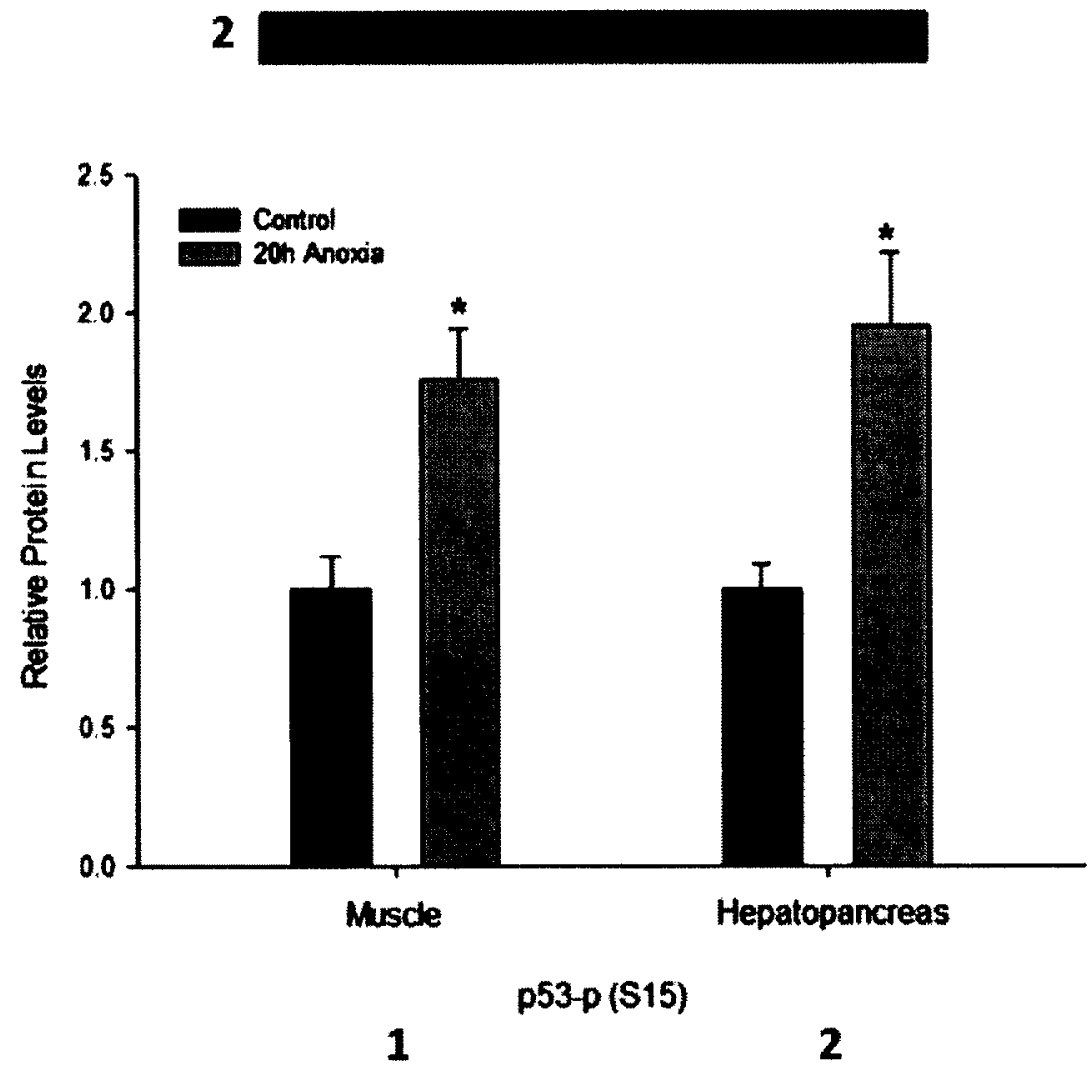

Figure 4.4: Effect of $20 \mathrm{~h}$ anoxic exposure on p53-p (Ser 15) activation in $O$. virilis tissues.

Changes in the phosphorylation of p53 at serine 15 measured in 'total' protein fractions in crayfish tissues in response to anoxia, as determined by western immunoblotting. Data are means $\pm S E M, n=4$ independent trials for muscle and hepatopancreas. * indicates significantly different from the corresponding control, $P<0.05$. 


\section{Control 20h Anoxia}

1

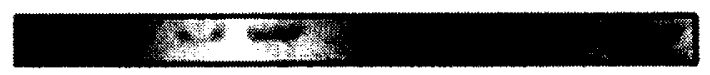

2

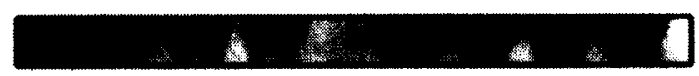

3

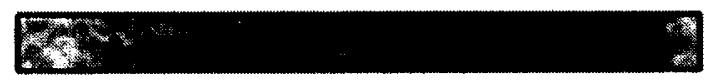

4
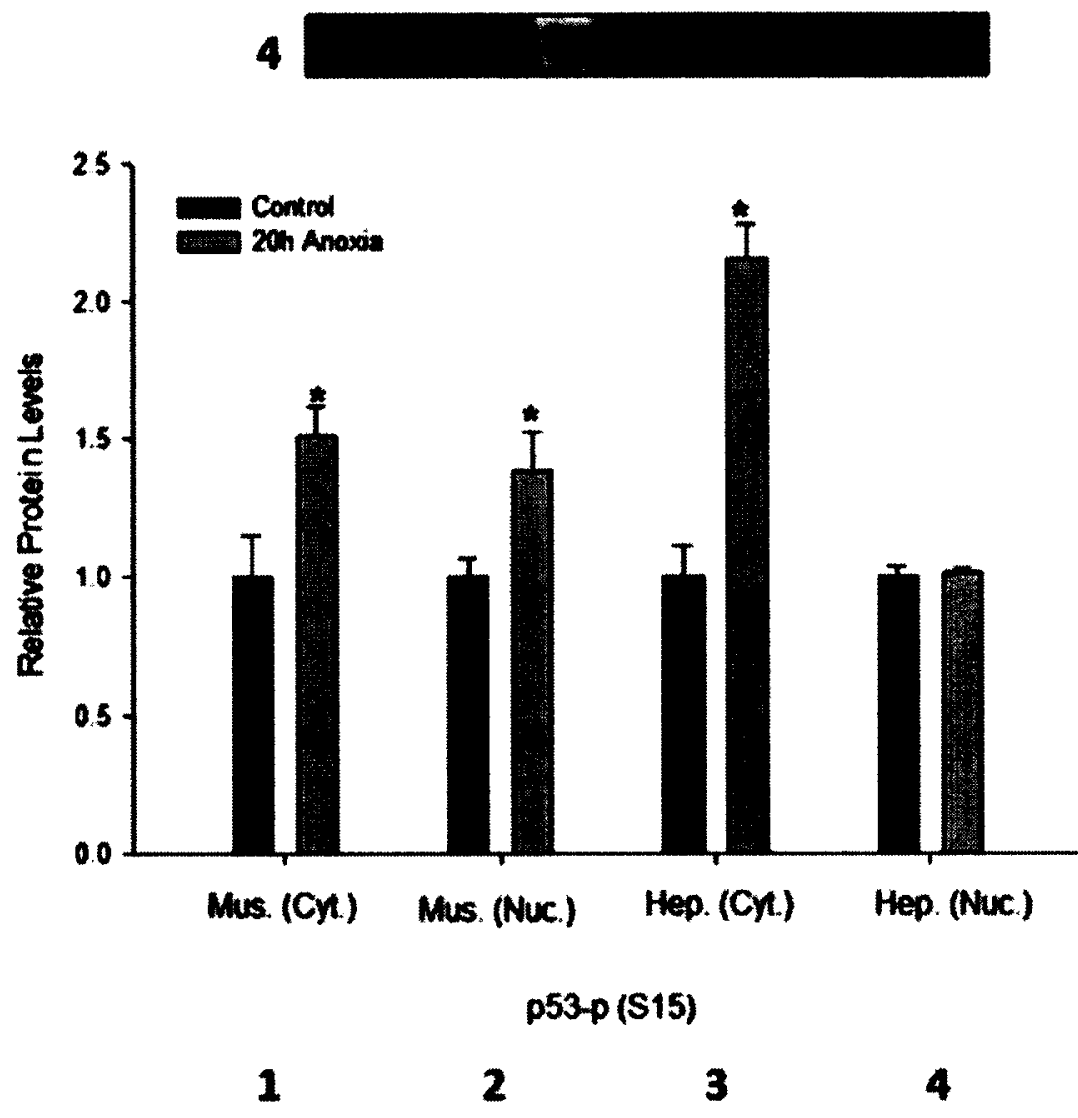

Figure 4.5: Effect of $20 \mathrm{~h}$ anoxic exposure on the localization of phosphorylated p53 in 0 . virilis tissues.

Changes in the relative phosphorylation of $\mathrm{p} 53$ at serine 15 in cytoplasmic and nuclear fractions of crayfish tissues in response to anoxia, as determined by western immunoblotting.

Data are means \pm SEM, $n=4$ independent trials for muscle and hepatopancreas. * indicates significantly different from the corresponding control, $P<0.05$. 

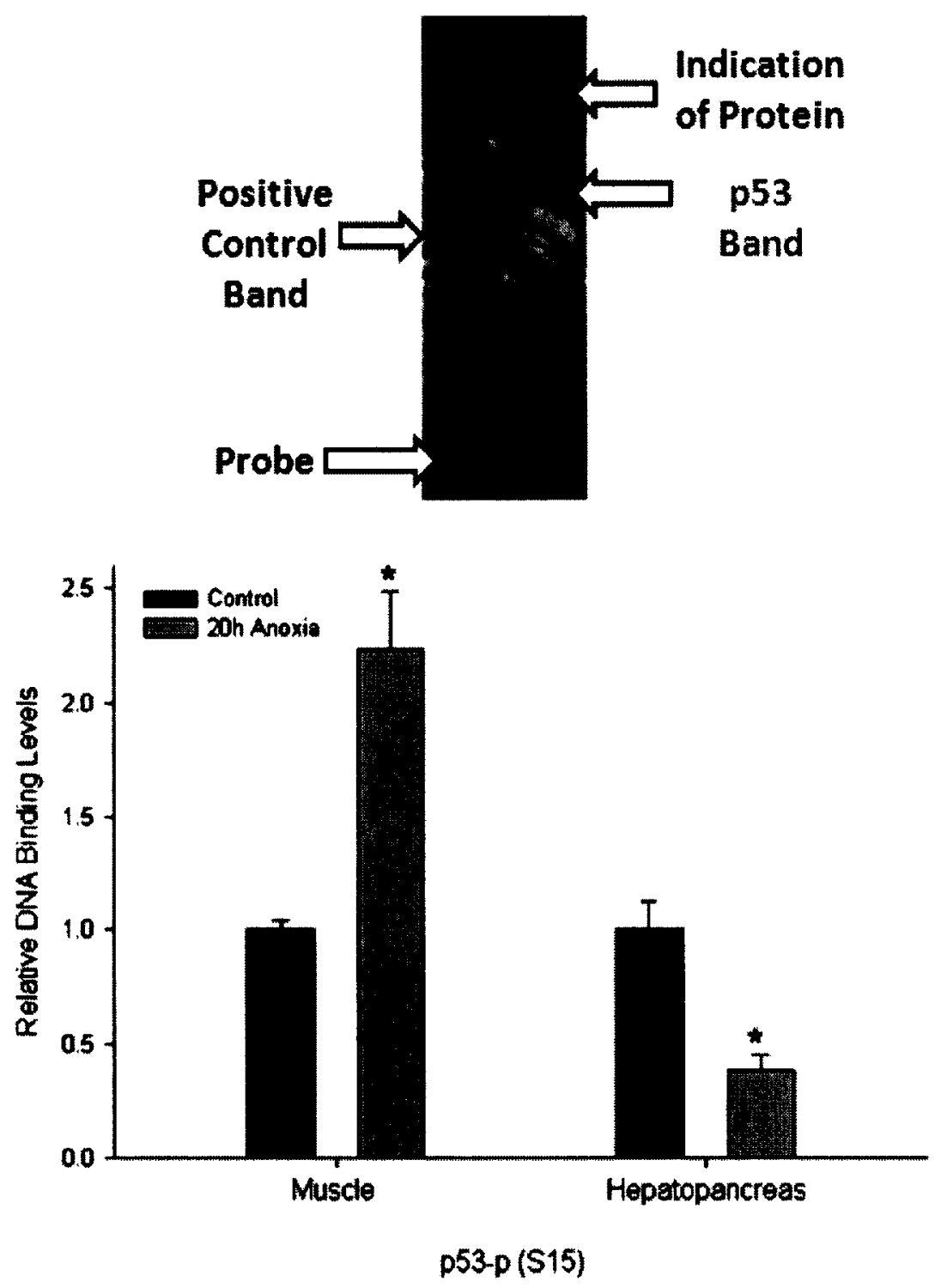

Figure 4.6: Effect of $20 \mathrm{~h}$ anoxia exposure on p53 DNA-binding activity in $O$. virilis tissues.

Changes in the DNA-binding activity of nuclear p53-p (S15) in the muscle and hepatopancreas in response to anoxia, as determined by a transcription factor binding assay. Data are means $\pm \mathrm{SEM}, n=4$ independent trials. * Indicates significantly different from the corresponding control, $P<0.05$.

Representative EMSA shown above the histogram. 


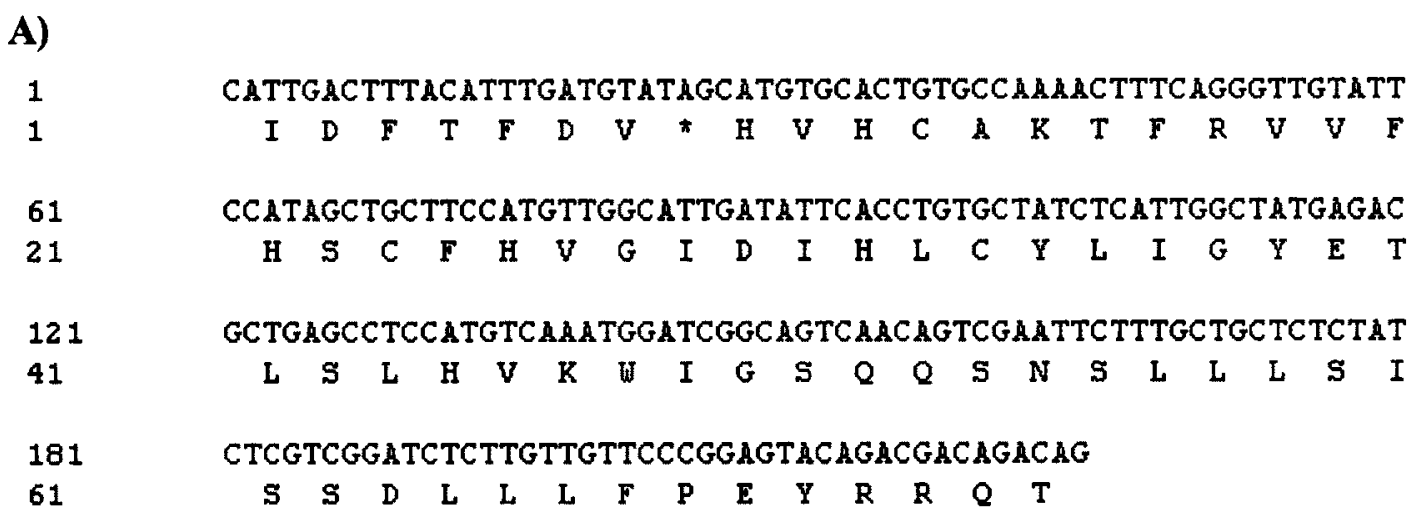

B)
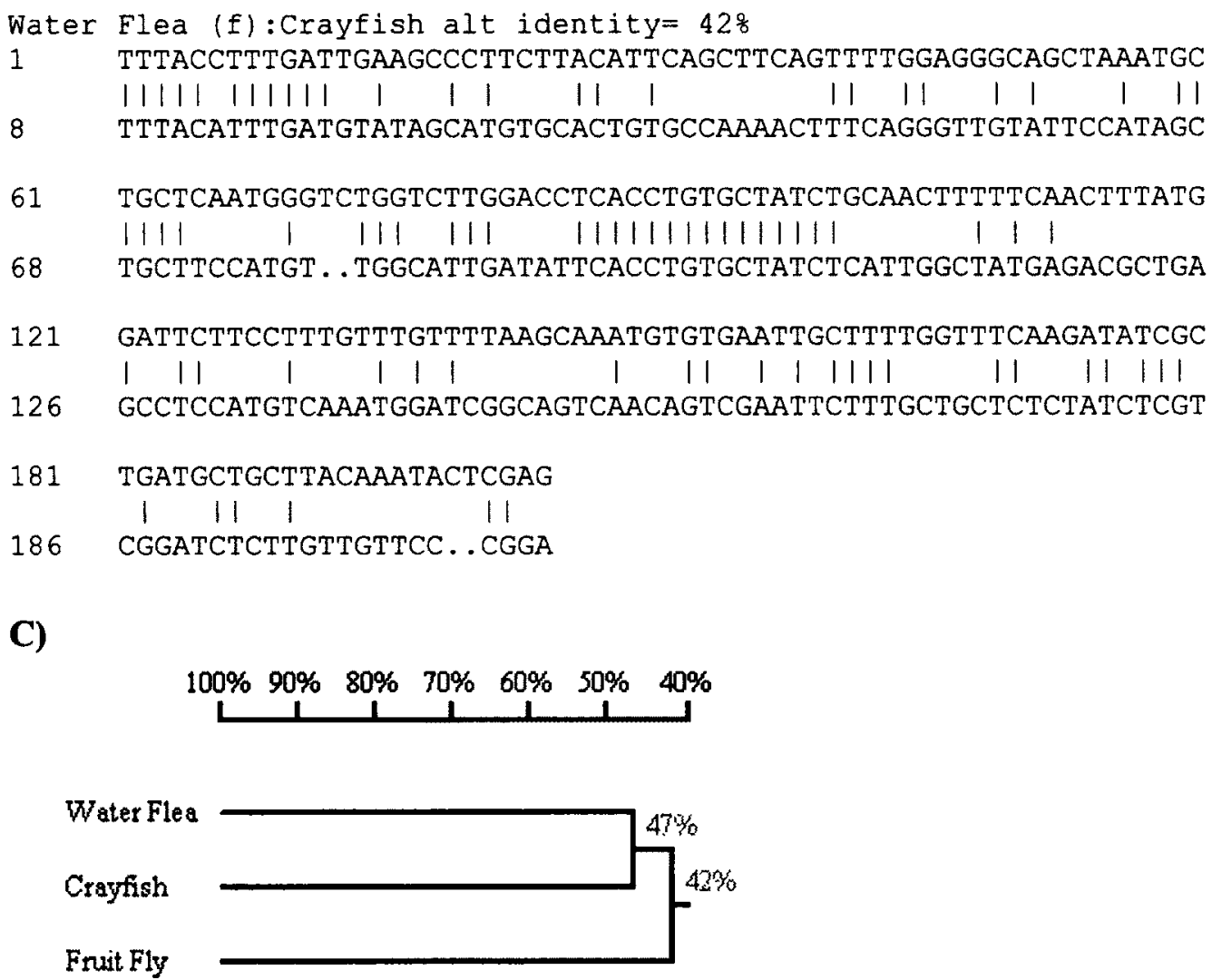

Figure 4.7: Partial nucleotide and deduced amino acid sequence of $O$. virilis ei24-like protein.

(A) The partial cDNA sequence of an ei24 like protein, with the corresponding amino acid sequence is shown. Nucleotides and amino acids are numbered on the left. The nucleotide sequence was 224 nucleotides in length, and encoded 73 amino acids.

(B) Sequence alignment of the isolated crayfish sequence and the corresponding fragment of the water flea sequence. 
(C) Homology tree produced from an alignment of the partial $O$. virilis ei24-like cDNA sequence with ei 24 from the water flea (Daphnia pulex) and fellow invertebrate arthropod, the fruit fly (Drosophila melanogaster). The accession for the fruit fly is AAF50975. There are currently no accession numbers for the sequenced $D$. pulex genes, but sequences may be found at http://wfleabase.org/. The percentage values correspond to the shared identity among the corresponding species. 

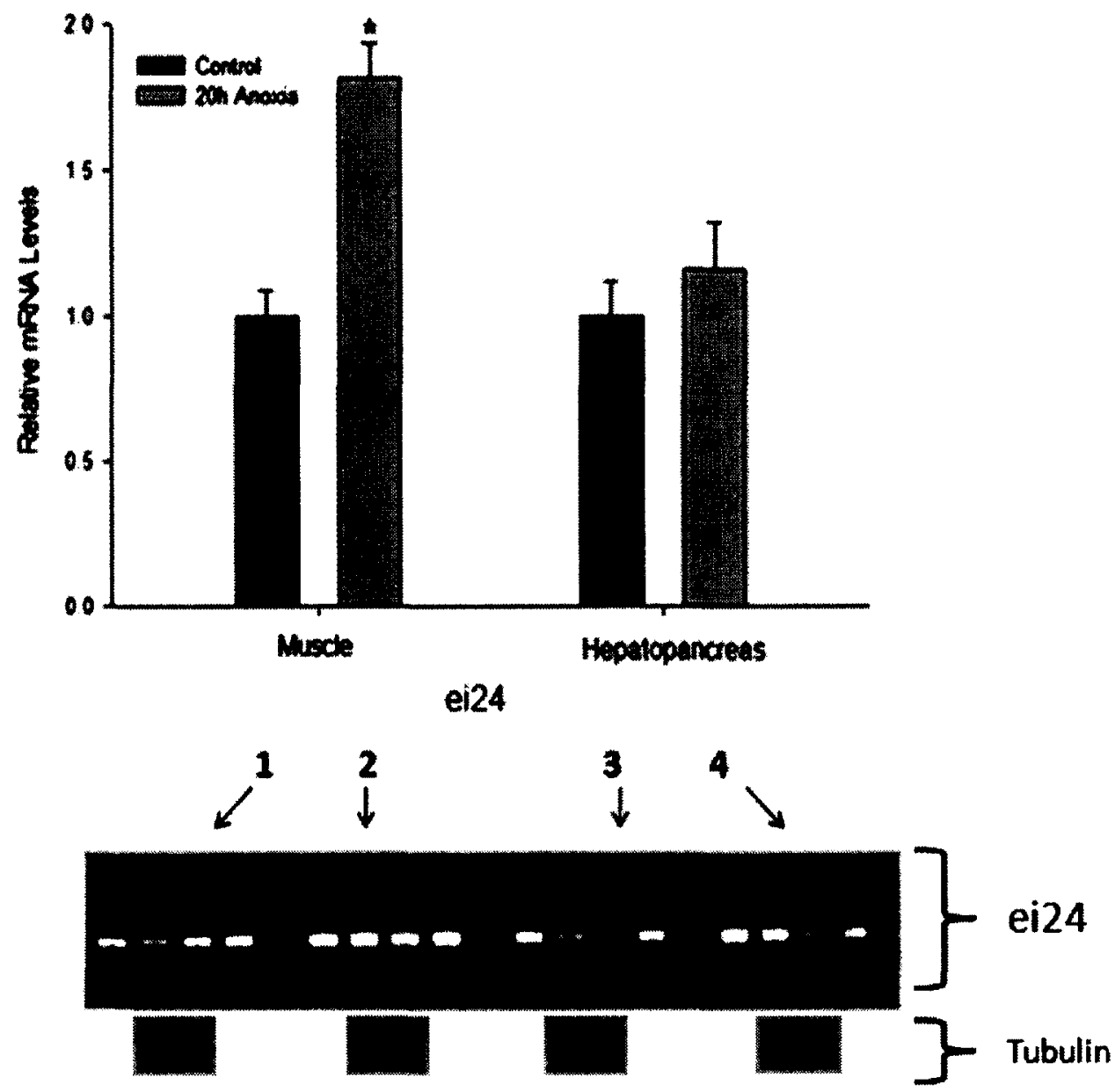

Figure 4.8: Effect of $20 \mathrm{~h}$ anoxic exposure on ei24-like transcript expression in $O$. virilis tissues as determined by RT-PCR.

Histogram shows the normalized transcript levels under control and anoxic conditions; bands were normalized against the corresponding $\alpha$-tubulin bands (representative from each sample shown) amplified from the same sample. Data are means \pm SEM, $n=4$ independent trials for mRNA isolated from different crayfish.

* Indicates significantly different from the corresponding control, $P<0.05$.

$1=$ control muscle, $2=20 \mathrm{~h}$ anoxic muscle, $3=$ control hepatopancreas, $4=20 \mathrm{~h}$ anoxic hepatopancreas

Representative ei24 amplicons shown below 

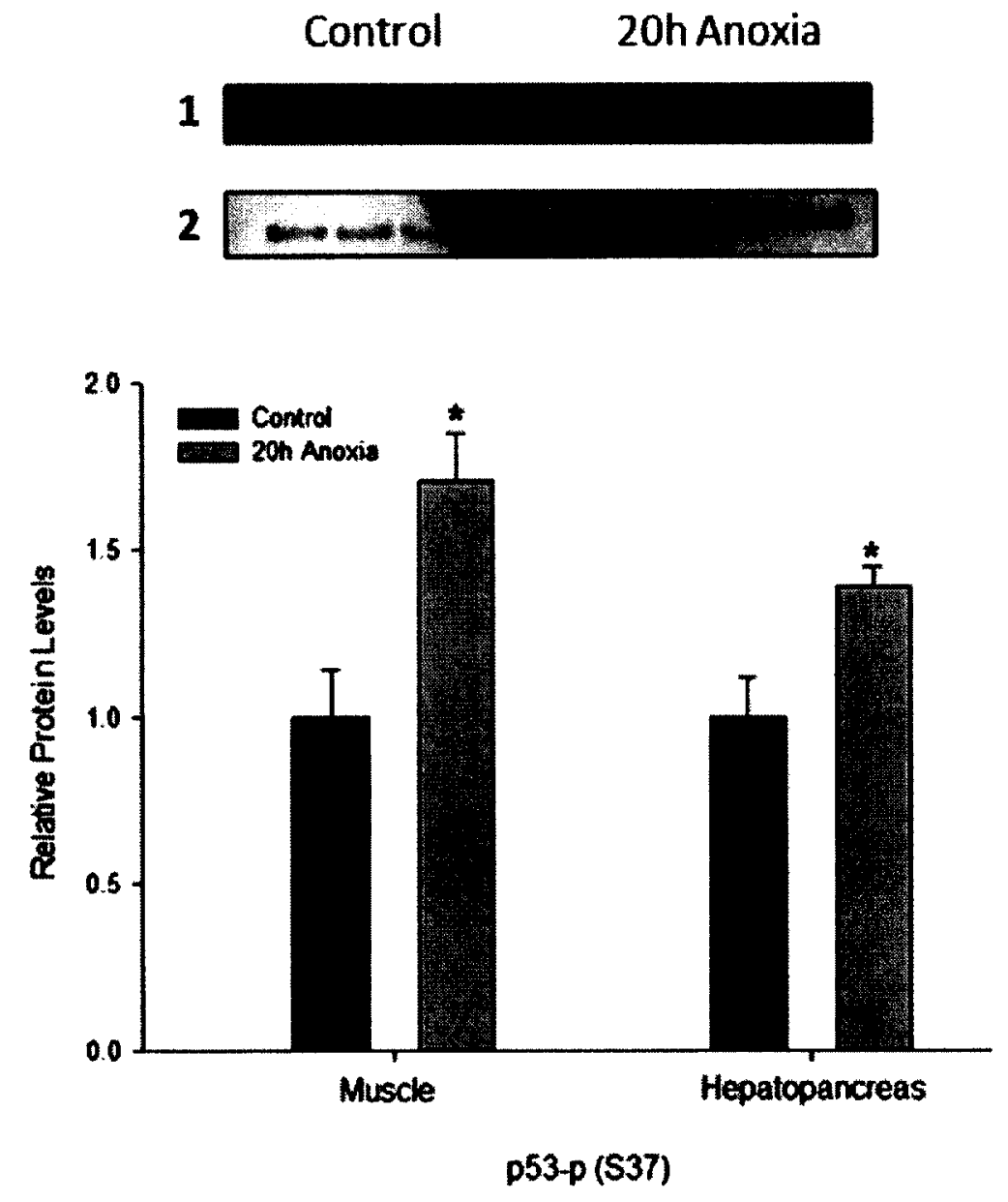

1

Figure 4.9: Effect of $20 \mathrm{~h}$ anoxia exposure on p53-p (Ser 37) activation in 0 . virilis tissues.

Changes in phosphorylation of p53 at serine 37 in 'total' protein extracts of crayfish tissues in response to anoxia, as determined by western immunoblotting.

Data are means \pm SEM, $n=4$ independent trials for muscle and hepatopancreas. * indicates significantly different from the corresponding control, $P<0.05$. 


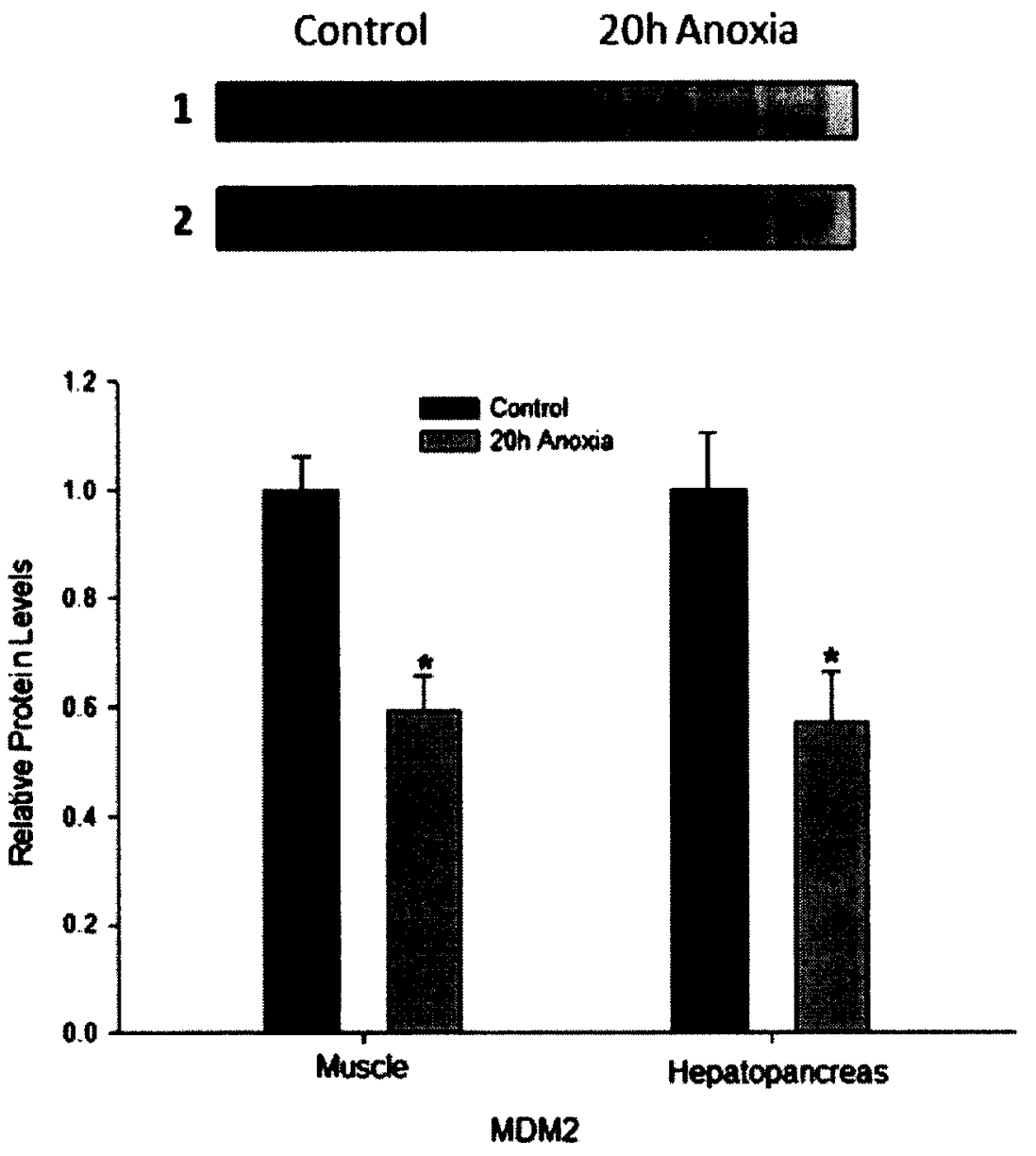

1

2

Figure 4.10: Effect of $20 \mathrm{~h}$ anoxic exposure on MDM2 protein levels in $O$. virilis tissues.

Changes in MDM2 protein levels in crayfish tissues in response to anoxia, as determined by western immunoblotting.

Data are means \pm SEM, $n=4$ independent trials for muscle and hepatopancreas. * indicates significantly different from the corresponding control, $P<0.05$. 

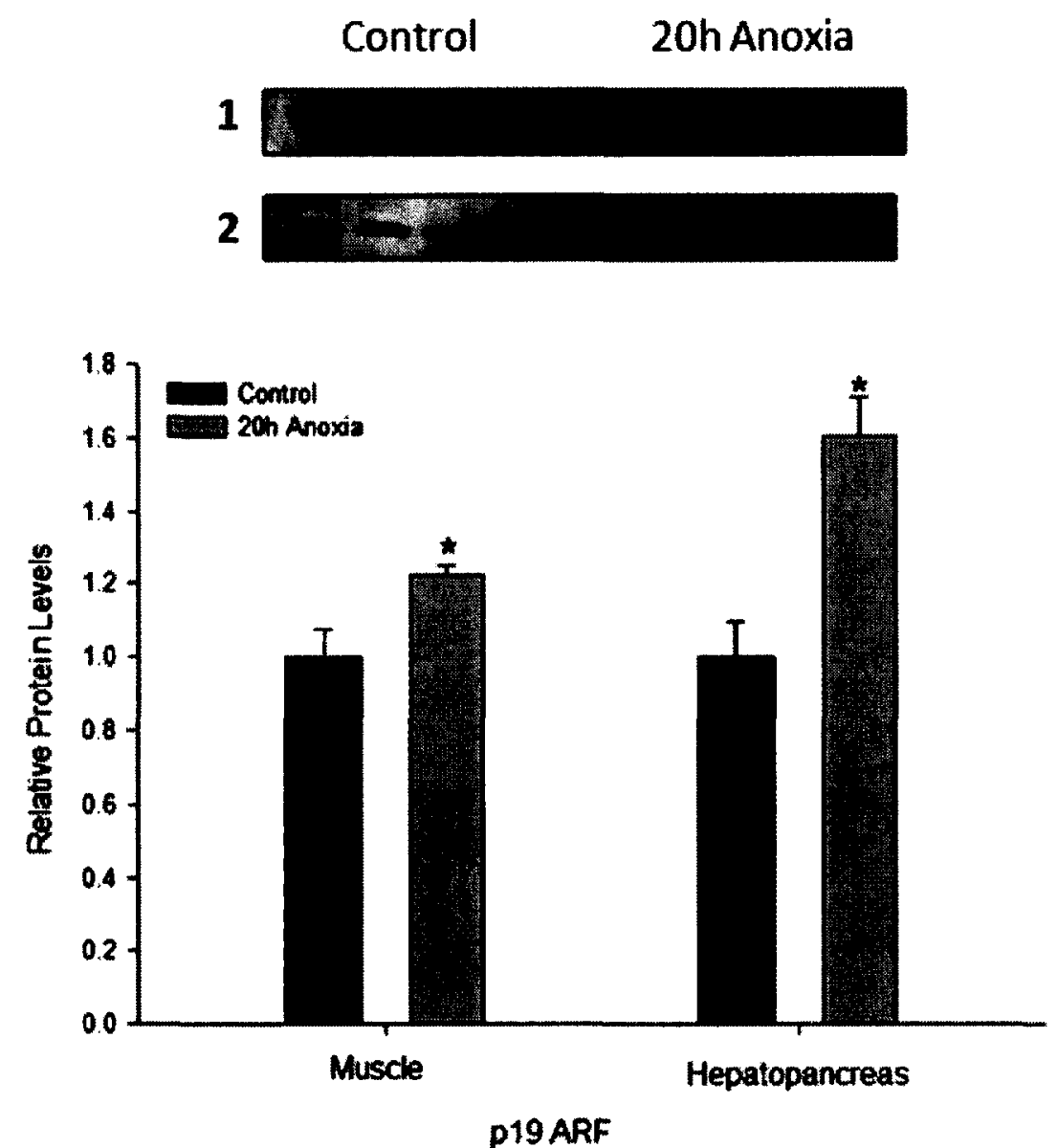

1

Figure 4.11: Effect of $20 \mathrm{~h}$ anoxic exposure on p19 ARF protein levels in $O$. virilis tissues.

Changes in p19 ARF protein levels in crayfish tissues in response to anoxia, as determined by western immunoblotting. Data are means $\pm S E M, n=4$ independent trials for muscle and hepatopancreas. * indicates significantly different from the corresponding control, $P<0.05$. 
Table 4.3 Chapter 4 data summary

\begin{tabular}{|l|l|l|l|l|l|l|l|l|l|l|}
\hline & \multicolumn{2}{l}{$\begin{array}{l}\text { Protein } \\
\text { (Total) }\end{array}$} & \multicolumn{2}{l|}{ Protein } & \multicolumn{2}{l|}{ H } & M & H & M & H \\
\hline & M & H & M & M & \multicolumn{2}{l|}{ ELISA } \\
& & & Cyto. & Nuc. & Cyto. & Nuc. & & & & \\
\hline ATM & $\uparrow$ & $\uparrow$ & & & & & & & & \\
\hline ATR & $\uparrow$ & $\uparrow$ & & & & & & & & \\
\hline p53 & $\leftrightarrow$ & $\leftrightarrow$ & & & & & & & & \\
\hline $\begin{array}{l}\text { p53-p } \\
\text { (S15) }\end{array}$ & $\uparrow$ & $\uparrow$ & $\uparrow$ & $\uparrow$ & $\uparrow$ & $\leftrightarrow$ & & & $\uparrow$ & $\downarrow$ \\
\hline ei24 & & & & & & & $\uparrow$ & $\leftrightarrow$ & & \\
\hline $\begin{array}{l}\text { p53-p } \\
\text { (S37) }\end{array}$ & $\uparrow$ & $\uparrow$ & & & & & & & & \\
\hline MDM2 & $\downarrow$ & $\downarrow$ & & & & & & & & \\
\hline p19 ARF & $\uparrow$ & $\uparrow$ & & & & & & & & \\
\hline
\end{tabular}

Note: Changes indicate statistical significance $(P<0.05)$; Western Blotting $(n \geq 4)$, RT-PCR $(n \geq 4)$, ELISA $(n=4)$

Arrows indicate changes or level profiles, in response to anoxia 


\section{Chapter 5}

Regulation of apoptosis and autophagy during anoxia in the freshwater crayfish $O$. virilis 


\subsection{Introduction}

Under prolonged periods of environmental stress, selective molecular signaling pathways determine survival based on energetic viability. In the anoxia resistant crayfish, $O$. virilis, this decision could be based on tissue type. Should selected cells not be able to sustain energetic homeostasis, programmed cellular death or apoptosis, may be signaled to destroy those cells while maintaining the viability of other cells. Alternately, stressed cells may undergo autophagy, a process of cellular minimization that recycles reusable cellular components. Unlike in their cellular roles as quality control mechanisms, both stress-induced apoptosis and autophagy are extreme responses, potentially resulting in significantly damaged or non-functional tissues. In the case of apoptosis, in the stress adapted crayfish, it is unlikely that under its standard anoxia triggered hypometabolic period that there would be significant levels of apoptosis; given its ability to resume a normal pattern of behavior following a recovery period. However, in an effort to protect vital organs (brain, heart, hepatopancreas) during the hypometabolic period, it might be expected that cells in less critical tissues such as skeletal muscle would be sacrificed to maintain the energetic homeostasis of the organism under a lengthened periods of hypometabolism.

Apoptosis and regulation by the Bcl family of proteins

The apoptosis machinery is constitutively expressed at a basal level in tissues and is primarily used to correct for mistakes made in cellular replication, particularly in embryonic cells (Kerr et al., 1972). However, when induced by genotoxic or environmental stress, apoptosis is capable of destroying not only individual cells but of spreading signals to surrounding cells that can lead to tissue, organ or even whole organismal death. In these conditions, apoptosis is primarily stimulated by DNA damage 
(Ferraro and Cecconi, 2007), often with signals arising from p53 (mentioned in the previous chapter), and is stimulated by outer mitochondrial membrane (OMM) permeability. OMM pores (OMMPs) formation allows release of second messengers such as cytochrome $\mathrm{c}$ and this causes downstream signaling to DNAases, endoribonucleases and cysteine proteases (caspase family) that stimulate rapid degradation of cellular components (Ferraro and Cecconi, 2007).

Regulating the integrity of the OMM is the B-cell lymphoma (Bcl) family of proteins (FIG 5.1). Bcl 1, 2 and 3 are all regarded as anti-apoptotic/pro-development protein families, with oncogenic capacity (Lishner et al., 1995). Within the Bcl-2 subfamily of proteins, there is a section of $\mathrm{Bcl}-2$ Homology $3(\mathrm{BH} 3)$ domain possessing members which are able to interact with and regulate each other (Smith et al., 2008). The Bcl-2 family contains both pro (Bax, Bak, Bim) and anti (Bcl-2, Bcl-xl) apoptotic members which are able to bind each other through their BH3 domains. Apoptosis depends on whether the pro-apoptosis members dimerize, resulting in apoptosis, or whether the pro and anti-apoptosis members dimerize, resulting in cellular survival. Autophagy and its regulation by TORCI

As an alternative to cell death, the process of autophagy may be enhanced under stress conditions. Autophagy is a cellular recycling procedure which 'minimizes' cell content while retaining viability as opposed to cell destruction via apoptosis (FIG 5.1). (Macro) autophagy relies upon the co-ordinated activities of numerous autophagy proteins (Atgs) to form an autophagosome; a lipid based cellular body that is able to integrate with lysosomes (Samara and Tavernarakis, 2008). The autophagosome section off portions of the cytosol, including damaged organelles and damaged/unfolded 
proteins, which are subsequently degraded by hydrolases when the autophagosome fuses with a lysosome to form an autophagolysosome (Samara and Tavernarakis, 2008). Autophagy is initiated by the Beclin 1 complex, which binds free cellular membrane. The ubiquitin-like conjugation and completion of the membrane is mediated by two main groups; the Atg5-12-16 complex and the Atg8 (LC3)-phosphatidyl ethanolamine (PE). Following the nucleation of the isolated membrane by the Beclin 1 complex, Atg5, Atg12 and Atg16 conjugate (via Atg7 and 10 activity) to expand the membrane. Atg8 (activated by cleavage from Atg4) is coupled to PE in a lipidation event which signals the maturation of the autophagosome (Eskelinen, 2008). Autophagy is a heavily regulated procedure, with extensive levels capable of triggering cellular death cascades, and as such, even the pro-survival Bcl family members have the capacity to inhibit autophagy, in a pro-survival mechanism.

When not induced by stress conditions, autophagy (like apoptosis) acts as a quality control repair mechanism, and may be induced (often by ROS damage) (Bergamini, 2006) to higher levels of activity that result in cellular minimization. While ROS damage during anoxia would be minimal, nutrient levels are also significant signals in triggering autophagy. Indeed, one of the major controlling elements of autophagy repression by the target of rapamycin (TOR) kinase - is primarily regulated by nutrient levels. In the yeast model, that has been instrumental in elucidating the autophagy mechanism, the TOR kinases are active under high nutrient conditions and actively repress autophagy (Yorimitsu and Klionsky, 2005). However, under nutrient limited conditions, when TOR is inactive, autophagic activity is increased significantly. 
TOR kinase signaling is pro-developmental and pro-survival, and is canonically activated through the similarly pro-developmental/survival PI3K/Akt signaling cascade. TOR is active in the form of two complexes, TORC1 and TORC2. Whereas TORC2 is primarily implicated in Akt activity (a positive feedback loop of TOR activity), TORC1 is the primary mediator of growth related processes such as protein translation, ribosome biogenesis and negative regulation of autophagy (Bai and Jiang, 2009). TORCl is comprised of the TOR kinase, the 'regulatory associated protein of mTOR' (raptor), and the G-protein subunit (GßL). The primary function of TORCl signaling is to facilitate protein synthesis, and it does so via controlling the activity of eukaryotic translation initiation factor $4 \mathrm{E}$ (eIF4E). TORC1 positively regulates protein translation by phosphorylating and inactivating the eIF4E repressor, eIF4E binding protein (4E-BP), and by activating (via phosphorylation) p70 S6 protein kinase (p70S6K) (Swiech et al., 2008). The p70S6K phosphorylates eIF4E allowing it to join its initiation complex, but is also able to positively regulate the eukaryotic elongation factor (eEF2), by repressing its inhibitor (via phosphorylation), the eEF2 kinase (eEF2K) (Swiech et al., 2008). Hence, p70S6K activity is a common signal of active TOR. Amongst the negative regulators of TORCl is the proline-rich Akt substrate 40 (PRAS40) which acts as a substrate inhibitor, by binding to raptor in the TORCl complex (Wang et al., 2007). Subsequently, PRAS40 may be inhibited by Akt phosphorylation, relieving TORC1 substrate binding capacity (Sancak et al., 2007).

TORC1 regulation of autophagy (FIG 5.2) centers on controlling the phosphorylation state of Atg13. Atg13 critically interacts with the Atg1 kinase in autophagosome formation, but as seen under nutrient rich conditions, TORCl mediated 
hyperphosphorylation of Atg13 severely reduces this interaction (Yorimitsu and Klionsky, 2005). Secondarily, TORCl competes for Vps34 (vacuolar protein sortingassociated protein 34; a class III PI3K kinase) which is implicated as a nutrient sensor (Pattingre et al., 2008). Vps34 is also required to form the Beclin 1 initiation complex (Pattingre et al., 2008), hence a competitive nature, based on nutrient availability arises between TORC1 pro-development signaling and autophagy.

In this chapter, both pro-survival $\mathrm{Bcl}$ and autophagy targets were analyzed in $O$. virilis, in response to anoxia. What arises is a tissue specific profile of both apoptosis and autophagy, with increases in both anti-apoptosis and autophagy expression in the tail muscle contrasted against a relatively unchanged (and even reduced in certain targets) profile of the same elements in the hepatopancreas. In the tail muscle, both pro-survival Bcls and autophagy targets indicated an increase in response to anoxia that was not contradicted by TOR profiling. Indeed, TOR (particularly TORC1) activity was also seen to correlate with autophagy; confirmed with both downstream (p70S6K) and inhibitor (PRAS40) activity profiles. In the hepatopancreas, however, there was a profile of stability throughout anoxia, with no significant changes in Bcl or autophagy protein levels, complemented by an increased TOR activity profile.

\subsection{Materials and Methods}

\subsubsection{Animals}

Crayfish were treated and tissue samples were collected as described in Chapter 2.

\subsubsection{Protein extracts and western blotting}

Protein extracts were prepared from tissue samples of control and $20 \mathrm{~h}$ anoxiaexposed crayfish. Equal amounts of protein $(20-30 \mu \mathrm{g})$ were loaded into each lane of 
$10 \%$ SDS polyacrylamide gels, electrophoresed and then electroblotted onto polyvinylidene difluoride (PVDF) membranes as described in Chapter 2. After the transfer of proteins, membranes were blocked for $15 \mathrm{~min}$ in Tris buffered saline containing Tween-20 (TBST: $20 \mathrm{mM}$ Tris base, $140 \mathrm{mM} \mathrm{NaCl}, 0.1 \% \mathrm{v} / \mathrm{v}$ Tween-20) with $2.5 \%$ non-fat dried milk added. Blots were probed overnight with primary antibodies (Table 5.1). All primary antibodies were diluted 1:1000 in TBST. After incubation, blots were washed several times with TBST and then incubated at RT for $1.5 \mathrm{~h}$ with HRPlinked goat anti-rabbit IgG (Cell Signaling, diluted 1:2000 in TBST). Immunoblots were developed using enhanced chemiluminescence.

\subsubsection{Immunoprecipitation}

Tissues were homogenized with a Dounce homogenizer at a 1:5 w:v in lysis buffer (10 $\mathrm{mM}$ Tris $\mathrm{HCl} \mathrm{pH} \mathrm{7.5,} 10 \mathrm{mM} \mathrm{NaCl}, 2 \mathrm{mM}$ EDTA, $0.1 \%$ Triton $\mathrm{X}$ and $1 \mathrm{mM}$ PMSF) to a final yield of $1 \mathrm{ml}$ total homogenate. Homogenates were incubated on ice for 20 minutes prior to centrifugation at $10,000 \times \mathrm{g}$ for 20 minutes $\left(4^{\circ} \mathrm{C}\right)$. Protein A agarose beads (Sigma) equilibrated in wash buffer ( $50 \mathrm{mM}$ Tris $\mathrm{HCl} \mathrm{pH} 7.5,150 \mathrm{mM} \mathrm{NaCl}$ and $0.05 \%$ Triton $\mathrm{X}$ ) were mixed with the lysate in a 1:20 v: $\mathrm{v}$ ratio and incubated for 120 minutes at $4^{\circ} \mathrm{C}$, with intermittent mixing. Following incubation, samples were centrifuged at 12,000 $\mathrm{x} g$ for 5 minutes. Protein content was measured using the Bio-Rad assay and then concentrations were normalized with addition of small aliquots of lysis buffer to a final concentration of 1 or $2 \mu \mathrm{g} / \mu 1$. Using $100 \mu 1$ of lysate, primary antibody (mTOR or GßL) was added 1:50 Ab:lysate and incubated overnight at $4^{\circ} \mathrm{C}$. Samples were centrifuged at $500 \mathrm{x} g$, supernatant was discarded and the pellet was resuspended in elution buffer $(62.5$ $\mathrm{mM}$ Tris $\mathrm{HCl} \mathrm{pH} \mathrm{6.8,10 \%} \mathrm{glycerol} \mathrm{and} \mathrm{2.3 \%} \mathrm{SDS).} \mathrm{Samples} \mathrm{were} \mathrm{prepared} \mathrm{for} \mathrm{and} \mathrm{run}$ 
through immunoblotting as above, loading $15 \mu \mathrm{l}$ sample per lane. Negative controls of protein incubated with beads alone, and protein incubated with bead and a non-specific (i.e. non target) antibody, were also created and run alongside the experimental samples.

\subsubsection{Data Analysis}

All data were analyzed as described in Chapter 2. 
Table 5.1 List of primary antibodies used in Chapter 5

\begin{tabular}{|l|l|l|}
\hline Primary Antibody & \multicolumn{1}{|c|}{ Company } & \multicolumn{1}{|c|}{ Catalogue \# } \\
\hline$B c l-2$ & $\begin{array}{l}\text { Cell Signaling (Danvers, MA, } \\
\text { USA) }\end{array}$ & $\# 2870$ \\
\hline$B c l-2-p(S 70)$ & $\begin{array}{l}\text { Cell Signaling (Danvers, MA, } \\
\text { USA) }\end{array}$ & $\# 2827$ \\
\hline$B c l-3$ & $\begin{array}{l}\text { Santa Cruz biotechnology (Santa } \\
\text { Cruz, CA, USA) }\end{array}$ & sc-13038 \\
\hline Bcl-xl & $\begin{array}{l}\text { Cell Signaling (Danvers, MA, } \\
\text { USA) }\end{array}$ & $\# 2764$ \\
\hline Beclin 1 & $\begin{array}{l}\text { Cell Signaling (Danvers, MA, } \\
\text { USA) }\end{array}$ & $\# 3495$ \\
\hline Atg5 & $\begin{array}{l}\text { Cell Signaling (Danvers, MA, } \\
\text { USA) }\end{array}$ & $\# 8450$ \\
\hline Atg7 & $\begin{array}{l}\text { Cell Signaling (Danvers, MA, } \\
\text { USA) }\end{array}$ & $\# 2631$ \\
\hline Atg8 & Abgent (San Diego, CA, USA) & AP1802b \\
\hline Atg8 (cleaved) & Abgent (San Diego, CA, USA) & AP3748a \\
\hline Atg12 & $\begin{array}{l}\text { Cell Signaling (Danvers, MA, } \\
\text { USA) }\end{array}$ & $\# 4180$ \\
\hline GBL & $\begin{array}{l}\text { Cell Signaling (Danvers, MA, } \\
\text { USA) }\end{array}$ & $\# 3227$ \\
\hline$m T O R-p$ (S2448) & $\begin{array}{l}\text { Cell Signaling (Danvers, MA, } \\
\text { USA) }\end{array}$ & $\# 5536$ \\
\hline $\begin{array}{l}\text { P70S6K-p } p \\
\text { (T421/424) }\end{array}$ & $\begin{array}{l}\text { Cell Signaling (Danvers, MA, } \\
\text { USA) }\end{array}$ & $\# 9204$ \\
\hline Pras40 & $\begin{array}{l}\text { Cell Signaling (Danvers, MA, } \\
\text { USA) }\end{array}$ & $\# 2610$ \\
\hline
\end{tabular}




\subsection{Results}

5.3.1 Protein expression and phosphorylation state of $B c l-2$ in response to anoxia

$\mathrm{Bcl}-2$ is a pro-survival member of the $\mathrm{Bcl} 2$ family of proteins, and in its phosphorylated form it is implicated in enhanced anti-apoptotic activity. Changes in the total protein levels of Bcl-2 and phosphorylation of serine 70 in response to anoxia were assessed using immunoblotting (FIG.5.3). The polyclonal Bcl-2 and Bcl-2-p (S70) antibodies cross-reacted with protein bands of $\sim 30 \mathrm{kDa}$ which is their expected size. In tail muscle, Bcl-2 protein levels increased by $1.8 \pm 0.2$ fold and phospho-Bcl2 (Ser 70) increased 1.3 \pm 0.08 fold under anoxia (both $\mathrm{P}<0.05$ ). In hepatopancreas, however, Bcl-2 protein levels and phosphorylation at serine 70 decreased under anoxia by $30 \pm 5 \%$ and $15 \pm 5 \%$, respectively $(\mathrm{P}<0.05)$.

\subsubsection{Protein expression of pro-survival Bcls (Bcl-3 and Bcl-xl) in response to anoxia}

Changes in the protein levels Bcl-3 and Bcl-xl, both pro-survival/anti-apoptotic members of the Bcl-2 family, in response to anoxia are shown in FIG 5.4. The polyclonal Bcl-3 and Bcl-xl antibodies cross-reacted with protein bands of $\sim 60$ and $30 \mathrm{kDa}$ which are their respective expected sizes. In the tail muscle, both $\mathrm{Bcl}-3$ and $\mathrm{Bcl}-\mathrm{xl}$ protein levels increased under anoxia by $1.6 \pm 0.2$ fold and $2.1 \pm 0.2$ fold, respectively (both $P<0.05$ ). In hepatopancreas, while Bcl-xl levels were unchanged under anoxia, Bcl-3 levels decreased significantly by $40 \pm 10 \%(\mathrm{P}<0.05)$.

\subsubsection{Protein expression of autophagy proteins in response to anoxia}

Autophagy involves the multi-step formation of the autophagosome including the combined activities of initiator (Beclin 1), conjugators (Atg5, Atg12, Atg8) and the conjugation activator Atg7. Protein levels of Beclin 1, Atg5, Atg12, Atg7, Atg8 (and its cleaved form) were measured in response to anoxia through immunoblotting. 
Polyclonal Beclin 1 antibodies cross reacted with bands of $\sim 60 \mathrm{kDa}$, which is their expected size. In tail muscle, protein levels increased significantly by $1.4 \pm 0.05$ fold $(P<0.05)$ under anoxia but remained static in the hepatopancreas (FIG 5. 5). Atg5 and 12 antibodies both cross reacted with bands of $\sim 55 \mathrm{kDa}$, which is their expected size. While Atg5 levels did not change in response to anoxia in either tissue, Atg 12 levels increased significantly in tail muscle by $1.8 \pm 0.1$ fold and decreased in hepatopancreas (FIG 5.6). In the tail muscle, Atg12 protein levels increased to over control values, and decreased in the hepatopancreas by $60 \pm 2 \%$ (both $\mathrm{P}<0.05$ ). Polyclonal Atg 7 antibodies cross reacted with bands of $\sim 80 \mathrm{kDa}$, which is their expected size. Similar to Atg12, Atg7 protein levels increased in tail muscle but decreased in hepatopancreas (FIG 5.7). Under anoxia, Atg7 protein levels rose by $2.4 \pm 0.08$ fold in tail muscle, but dropped by $25 \pm 6$ $\%$ in hepatopancreas.

Atg8, in order to be activated, must undergo post-translational cleavage of the Cterminal amino acid residue by Atg4. Following this cleavage, Atg8 can be coupled to phosphatidyl ethanolamine as part of the autophagosome formation mechanism.

Polyclonal antibodies detecting Atg8 and its cleaved form (Atg8 cl.) both cross reacted with bands of $\sim 15 \mathrm{kDa}$, their expected size. In tail muscle, both Atg8 and Atg8 $\mathrm{cl}$. increased significantly under anoxia (FIG 5.8). Atg8 protein levels increased $2.0 \pm 0.2$ fold, whereas the cleaved form increased $1.5 \pm 0.1$ fold (both $\mathrm{P}<0.05$ ). In hepatopancreas, however, there were no significant changes to either form.

\subsection{4 mTOR/GBL protein expression and TORC incorporation in response to anoxia} TOR complexes contain both mTOR, G $\beta \mathrm{L}$ and either raptor (C1) or rictor (C2). The TOR complexes are the effectors of TOR signaling, targeting growth and development $(\mathrm{C} 1)$ 
and Akt (C2) signaling downstream. Amongst the targets of TORC1 are autophagy proteins (Atg13) and protein translation activators (p70S6K). Protein levels of mTOR (FIG 5.9) and G $\beta$ L (FIG 5.10) as well as their speculated inclusion in TORC, in a higher molecular weight complex, were analyzed via western blotting. MTOR and GßL polyclonal antibodies cross reacted with bands of $\sim 290 \mathrm{kDa}$ and $40 \mathrm{kDa}$ respectively, their expected sizes. The speculated TORC complex was located significantly above both bands, and cross reacted with both sets of antibodies.

In the tail muscle, there were no significant changes in mTOR protein levels, but $\mathrm{G} \beta \mathrm{L}$ and its presence in TORC showed significant decreases under anoxia (by $30 \pm 5 \%$ and $40 \pm 2 \%$,respectively (both $\mathrm{P}<0.05$ ). In the hepatopancreas, while there were no changes in either mTOR or G $\beta L$ protein levels, TORC expression seemed to increase

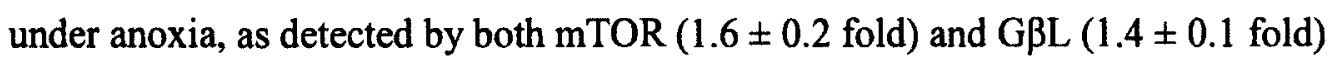
antibodies (both $\mathrm{P}<0.05$ ).

\subsubsection{Phosphorylation of PRAS40 and p70S6K in response to anoxia}

Both PRAS40 and p70S6K phosphorylation states are indicative of TORC1 activity. PRAS40, a TORC1 inhibitor is unable to do so when phosphorylated (primarily by Akt), and $\mathrm{p} 70 \mathrm{~S} 6 \mathrm{~K}$ may be targeted directly by mTOR for phosphorylation in order to allow protein translation. Phosphorylation of threonine 246 on PRAS40 and threonine 421/424 on p70S6K was assessed in both tissues, in total protein fractions in response to anoxia (FIG 5.11). Polyclonal PRAS40-p (T246) and p70S6K-p (T421/424) antibodies cross reacted with bands of $\sim 40$ and $80 \mathrm{kDa}$, which are their expected sizes.

In tail muscle, phosphorylation of both PRAS40 and p70S6K were stable between aerobic and anoxic states. In hepatopancreas, however, phosphorylation levels of both 
proteins increased under anoxia. PRAS40 phosphorylation levels increased $2.5 \pm 0.2$ fold, whereas p70S6K phosphorylation increased $1.5 \pm 0.08$ fold; both $P<0.05$.

\subsection{Discussion}

The control of both apoptosis and autophagy is often mediated by the same pro-survival upstream signaling, but despite this, one may be selected over the other depending on the stimulus. Although present at basal levels in unstressed cells, apoptosis is not a common response to environmental stress conditions in species that can use reversible metabolic rate depression to endure prolonged stress. The reason for this may be that apoptosis is a one-way destruction of cells whereas autophagy allows cells to reduce cellular metabolism to a "skeleton staff", recycling cellular components to produce building blocks and energy for central vital functions and providing for cellular recovery at the end of the stress period. In the nematode C. elegans, for example, induction of autophagy is critical to dauer survival through the period of quiescence (see Appendix A) (Mörck and Pilon, 2007). This is also the path taken by tumor cells when challenged by hypoxia due to a lack of developed vasculature (Rouschop and Wouters, 2009). The important separation between signaling of the two processes may lie in the level of oxidative damage. While apoptosis is primarily triggered by oxidative stress, via DNA damage sensing machinery, autophagy is primarily responsive to nutrient starvation. Hence, in autophagy, cellular components are broken down to provide precursor molecules and nutrients for other cells (Yorimitsu and Klionsky, 2005). In the context of anoxic survival, autophagy is the more applicable of the two processes, but it is interesting nonetheless to analyze the response of the anti-apoptotic proteins. Furthermore, the role of TOR, and specifically TORC1 shows a division between pro-developmental/pro- 
survival signaling through its activity on p70S6 $\mathrm{K}$ (protein translation) and Beclin 1 (autophagy), respectively, and its activity profile during hypometabolism is therefore of great interest.

From the perspective of whole organism survival, there is often a hierarchy among tissues that is established to prevent irreversible damage over a prolonged stress period. Indeed, the crayfish $O$. virilis, in one of its physiological adaptations to hypoxia, preferentially shifts hemolymph to the anterior portion of its body (brain, heart, hepatopancreas) to allow its circulation to support oxygen and nutrient delivery to critical core organs for as long as possible (Reiber, 1995). In the event of severe or prolonged energetic debt, organs of lesser importance and/or with greater metabolic capacity for long term anaerobic survival (e.g. tail muscle can draw upon high arginine phosphate and glycogen reserves to fuel a minimum metabolic rate), may be "sacrificed" in order to keep remaining supplies of precursors/nutrients/oxygen for other organs in an attempt to maintain energetic homeostasis and critical organ viability for as long as possible. Hence survival over a prolonged anoxic exposure would not only require the suppression of apoptotic signaling, but the potential energetic compromise that would arise in autophagy signaling.

$B C L$ expression under anoxia

Regulating the progression or repression of apoptotic signaling are the Bcl protein family members; Bcll, 2 and 3 . Bcl-3 has distinct activity, separate from the Bcl-2 family that is typically associated with apoptosis regulation, but is nonetheless a B-celllymphoma protein that maintains oncogenic (i.e. anti-apoptotic - pro-survival/ proliferation) activity. It contains structural similarities to the IKB family of proteins, and 
acts as a differential regulator of NF-kB transcription factors (Dechend et al., 1999).

Within the Bcl-2 subfamily, Bcl-2 and Bcl-xl interact with the pro-apoptosis Bcl-2 members via their $\mathrm{BH} 3$ domain. In the present analysis of $O$. virilis, the protein expression of the pro-survival members ( $\mathrm{Bcl}-2, \mathrm{Bcl}-3$ and $\mathrm{Bcl}-\mathrm{xl})$ begins to suggest a tissue specific response to anoxia (Table 5.2). In the hepatopancreas, there was a decrease in Bcl-2 total protein and phosphorylation of serine 70 as well as a decrease in Bcl-3, and a stable profile of $\mathrm{Bcl}-\mathrm{xl}$. This may indicate suppression in terms of energetically expensive protein synthesis, but more likely is indicating that the anti-apoptosis proteins are not needed. Their suppression in terms of both total protein levels and phosphorylation in the case of Bcl-2, would indicate in the absence of apoptosis, a lack of interaction with the pro-apoptotic members. This may also translate into a lack of Bcl regulation of autophagy, or indeed its necessity in the hepatopancreas. By contrast, tail muscle exhibited increased protein levels of all three anti-apoptotic proteins (Bcl-2, Bcl-3 and $\mathrm{Bcl}-\mathrm{xl}$ ), along with increased phosphorylation of $\mathrm{Bcl}-2$. While the increased phosphorylation of Bcl-2 may just be a consequence of increased total protein, phosphorylation of Bcl-2 is known to confer greater anti-apoptotic capacity (Deng et al., 2001). Stress activated protein kinases, such as the c-Jun N-terminal kinase (JNK) and the extracellular signal-regulated kinase (ERK1/2), are capable of phosphorylating Bcl-2 at serine 70, under conditions of toxicity (Deng et al., 2001). While the mechanism of how phosphorylation enhances Bcl-2 activity is largely unknown, it is speculated that phosphorylation both reduces proteasomal degradation of Bcl-2 (Dimmeler et al., 1999) and stabilizes the Bcl-2/Bax association (Deng et al., 2000) to prolong mitochondrial membrane stability through stress periods. Hence, it may follow that increased 
phosphorylation of Bcl-2, along with increased protein levels of all three pro-survival Bcls under anoxia, is a response of a tissue potentially more susceptible to apoptosis signaling under the stress period.

Autophagy expression under anoxia

A metabolic function that is conserved from yeast to mammals, autophagy is a coordinated process that involves upwards of 32 autophagy specific proteins. In the process of autophagosome formation, there are three critical steps; initiation (Beclin-1 complex mediated), conjugation and elongation (Atg5/12/16 and Atg8/PE mediated). The PI3K like initiation complex is typically comprised of Beclin 1, Vps34, the regulatory myristoylated kinase Vps15 and the UV resistance associated gene protein (UVRAG), although depending on function may include other components such as Atg14 (He and Levine, 2010). Beclin 1, and its capacity to form the initiation complex, is one of the key regulatory switches needed to allow or inhibit autophagy. However, approximately half of autophagy proteins are involved in the formation of the conjugation complexes of Atg12-5 and Atg 8. Atg12 is activated by Atg 7, transferred to Atg10, and subsequently conjugated to Atg 5 prior to conjugation to Atg16 (Hanada et al., 2007). The formation of this conjugate takes place prior to the lipidation of Atg8 (by coupling to PE), and the absence of Atg12-5 can drastically limit Atg8 activity downstream (Hanada et al., 2007). The Atg12-5 conjugate acts as an E3 ubiquitin ligase in the formation of the Atg8-PE conjugate, which promotes the expansion of the autophagosome membrane, and is necessary for efficient lipidation of Atg8 after Atg8-Atg3 complex formation, indicating its role in transfer of Atg8 from Atg3 to PE (Hanada et al, 2007). Atg8 itself acts like ubiquitin, and once processed (cleaved C-terminal glycine) by Atg 4, and conjugated to 
PE, and localizes to the phagophore assembly site (Xie et al., 2008). Indeed, Atg8 is speculated to regulate the size of the autophagosomes, and hence the level of autophagy rather than the frequency of autophagy (i.e. the number of autophagosomes) (Xie et al., 2008). Atg8 is amongst the most common markers of active autophagy, and elevated protein levels are seen in starvation-induced autophagy (Huang et al., 2000).

In crayfish tail muscle (Table 5.2) there was strong evidence that autophagy was induced during anoxia, possibly in conjunction with the nutrient deprivation associated with this period of hypometabolism. Increased protein levels of Beclin-1, Atg 12 (but not Atg5), Atg7 and Atg8 (along with parallel increase in Atg8 cleaved form) were all markers that indicated stress induced autophagy (preparation for this event) in muscle. However, in hepatopancreas, expression patterns of autophagy associated proteins were consistent with a profile of no increase in autophagy under anoxia. Indeed, in a suspected pattern of cellular protection, there were in fact decreases in autophagy proteins, Atg 12 and its activator, Atg7, along with no changes in Beclin 1, Atg5 or 8 in the hepatopancreas. This would indicate that any autophagy during anoxia in the hepatopancreas was strictly at basal levels, and was not enhanced by anoxia stress, as it appeared to be in tail muscle.

Regulating autophagy

The $\mathrm{Bcl} 2$ family proteins (Bcl-2 and $\mathrm{xl}$ ), along with their role in mediating mitochondrial stability, also have the capacity to regulate autophagy through Beclin 1 binding. In much the same way that they are able to inhibit Bak and Bax, these Bcls prevent Beclin-1 from entering its autophagy initiation complex (with Vps34 and UVRAG), by virtue of binding to the BH3 domain of Beclin 1 (Maiuri et al., 2007). 
Subsequently, binding of Bcl-2 and Bcl-xl by other $\mathrm{BH} 3$ members would prevent their inhibition of Beclin-1, indicating a parallel regulation step for both pro-apoptosis and pro-autophagy signaling. Interestingly, along with Bcl-2 binding, phosphorylation affects the capacity of Bcl-2 to bind Beclin-1. Nutrient starvation, in its capacity to trigger autophagy, is seen to also be linked with Beclin-1 dissociation from its inhibitors. One of the mechanisms that is suspected to result in increased free Beclin-1 protein is phosphorylation of Bcl-2 by JNK (Levine et al., 2008). Hence the same signal that increases Bcl-2 anti-apototic function also decreases Bcl-2 anti-autophagy dissociation. JNK inhibition, under nutrient starvation conditions, decreased Bcl-2/Beclin-1 dissociation, implicating phosphorylation by JNK as a pro-autophagy response mechanism (Levine et al., 2008). This may intimate a dual role for the profile of $\mathrm{Bcl}-2$ phosphorylation seen in the tail muscle of $O$. virilis. While the expression profiles of both increased Beclin-1 and Bcl-2 protein levels could indicate least a partial 'cancelling out' of autophagy up-regulation ( via Bcl-2 (and Bcl-xl) inhibition of Beclin-1), concurrent increased Bcl-2 phosphorylation may suggest an opening for both Beclin 1 activity and anti-apoptotic Bcl-2 activity.

Bcl-xl is also implicated in autophagy control, but in conjunction with a tumor suppressor protein. The p19 ARF tumor suppressor (ARF), known to have apoptotic activity by itself or through MDM2 repression (allowing tumor suppressor p53 activity), has recently been discovered as having a novel role in autophagy stimulation (Pimkina et al., 2009). Independent of its pro-apoptosis role, ARF is thought to induce autophagy by acting as a binding protein for Bcl-xl, reducing complex formation between $\mathrm{Bcl}-\mathrm{xl}$ and Beclin-1 (Pimkina et al., 2009). Hence in O. virilis, increased p19 ARF levels under 
anoxia (shown in the previous chapter) in conjunction with increased Bcl-xl protein in tail muscle, may suggest that free Beclin 1 is allowed to propagate autophagy during anoxia-induced hypometabolism.

The most widely recognized regulator of autophagy, however, is TOR; specifically complex 1 (TORC1). TORC1, as part of its pro-developmental role typically keeps autophagy in check in unstressed cells, but as in the case of C. elegans, environmental stress may cause a drop in TOR activity, subsequently enhancing autophagy (see Appendix A) (Mörck and Pilon, 2007). TORCl negatively regulates the activity of Atgl kinase by either direct or indirect hyperphosphorylation (the mechanism is unclear) of Atg13 (a regulatory subunit of Atg1), and in doing so alters the standard autophagy path of switching cytoplasm-to-vacuole targeting (Cvt) transport (vacuoles in the yeast model, lysosomes in the animal model) into autophagosome formation (DíazTroya et al., 2008). Atg1 is active both as a kinase and as part of a complex involved in the structural formation of the autophagosome. In its complex (with Atg17, 11, 13 and Vac8) Atg1 physically interacts with Atg1 1 and 17, leaving Atg13 to interact with Vac8; TORC1 activity primarily maintains low Atg13 affinity for Atg1. While the full mechanisms of TOR based autophagy repression have not been elucidated, transcript levels of Atgs (including 1,3,4,5,7,12,and 13) are responsive to TOR as shown by rapamycin treatments which inhibit TOR activities (Díaz-Troya et al., 2008). Vps34, the class III PI3K, also bridges TOR and autophagy activity. Vps34, in a mechanism linked to the availability of amino acids, is thought to activate TORC1 under high amino acid conditions (Nobukuni et al., 2007), but under limiting conditions is able to join in the Beclin 1 complex to promote autophagy. Hence, mTOR activity, which is closely tied to 
the negative regulation of autophagy, could indicate further tissue specific responses to anoxia in crayfish. Immunopreciptation of TORC members, mTOR and G $\beta L$, in the tail muscle showed either a level profile (mTOR) or decreased profile (G $\beta L$ and TORC), which corresponds with the elevated autophagy profile in muscle during anoxia. In the hepatopancreas, level profiles of G $\beta \mathrm{L}$ and $\mathrm{mTOR}$ in conjunction with an increase in (potential) TORC expression suggest a more active TORC profile under anoxia, which likewise correlates with the reduced autophagy presence.

To gain a fuller picture of TOR activity (Table 5.2), phosphorylation of the downstream target, p70S6K was shown to be unaffected in tail muscle of anoxic animals, whereas it increased significantly in the hepatopancreas, further correlating with the TOR activation profile. P70S6K is a crucial pro-translation target, allowing eIF4E to initiate translation, and is one of the primary markers of active TORC1. Subsequently, levels of PRAS40 phosphorylation were also measured. PRAS40, a pseudo substrate inhibitor of TORCl (specifically binding raptor), is insulin signal regulated, being a target of Akt repression. Akt, the downstream kinase of insulin signaling, and activator of TORC1 through TSC1/2 repression, is also able to phosphorylate PRAS40 at the threonine 246 residue, allowing TORCl activity as seen through S6K activity (Sanack et al., 2007). Holding the pattern of the previous results, PRAS40 phosphorylation was unchanged in the tail muscle of $O$. virilis through anoxia, but rose in the hepatopancreas together with a greater TORC activity during anoxia.

\section{Conclusions}

In assessing the regulation of apoptosis and autophagy during anoxia in $O$. virilis, a tissue specific pattern becomes apparent. This was, to a degree, expected, and the trends 
exhibited in the tail muscle (Table 5.2) run parallel to the hypothetical predictions made for both apoptotic defense and autophagy induction.

Increased Bcl expression to repress apoptosis, in conjunction with increased autophagy protein levels indicate that tail muscle may be susceptible to energetic limitations under anoxia; indeed this mechanism relates favourably to the dauer mechanism for survival (see Appendix A). Driven by nutrient levels, autophagy induction is well documented, and under anoxia the combined activation profile of the Atgs, along with critical inhibitors (Bcl-2, Bcl-xl and mTOR) all potentially show evidence of positive regulation of the autophagy mechanism. This opens the possibility to muscle cells being sacrificed as sources of nutrients/precursors to promote energetic homeostasis during hypometabolism. The hepatopancreas, conversely, showed little or no evidence of apoptosis or autophagy induction under anoxia. Hepatopancreas TOR activity profiling showed signs of active TORC, which may account for the repressed autophagy profile. Autophagy (or apoptosis) of the hepatopancreas, at a stress induced level could be potentially fatal to the organism as a whole, given the importance of the organ. Hence the dual regulation of autophagy may suggest a novel mechanism of survival under oxygen, energy and nutrient limitation conditions that are occur when crayfish are exposed to anoxic conditions. 


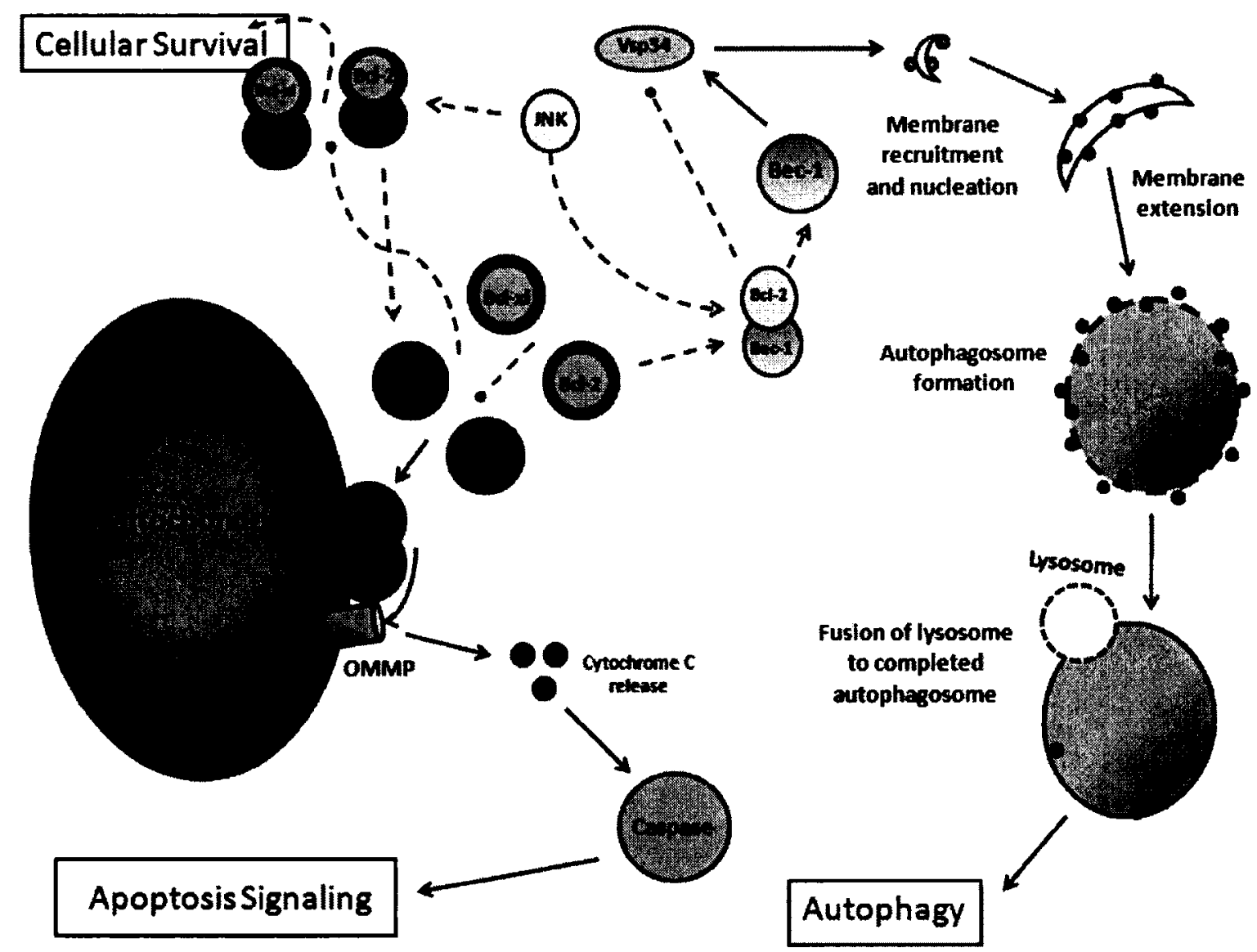

Figure 5.1: An overview of apoptosis and autophagy regulation. In apoptosis regulation, formation of the outer mitochondrial membrane pore (OMMP) is the primary step. Pro (Bax/Bak) and anti (Bcl-xl, Bcl-2) apoptosis members of the Bcl-2 family can interact to form functional homo or heterodimers to promote or prevent apoptosis, respectively. Autophagy, initiated by the Beclin 1 complex, can also interact with the Bcl-2 family. Through the initiation of autophagosome formation, Atgs 5, 12 and 16 conjugate to extend the membrane. Atg8 is cleaved and lipidated to finalize the autophagosome. This body, encompassing portions of the cytoplasm, fuses with a lysosome which subsequently degrades the contents. 


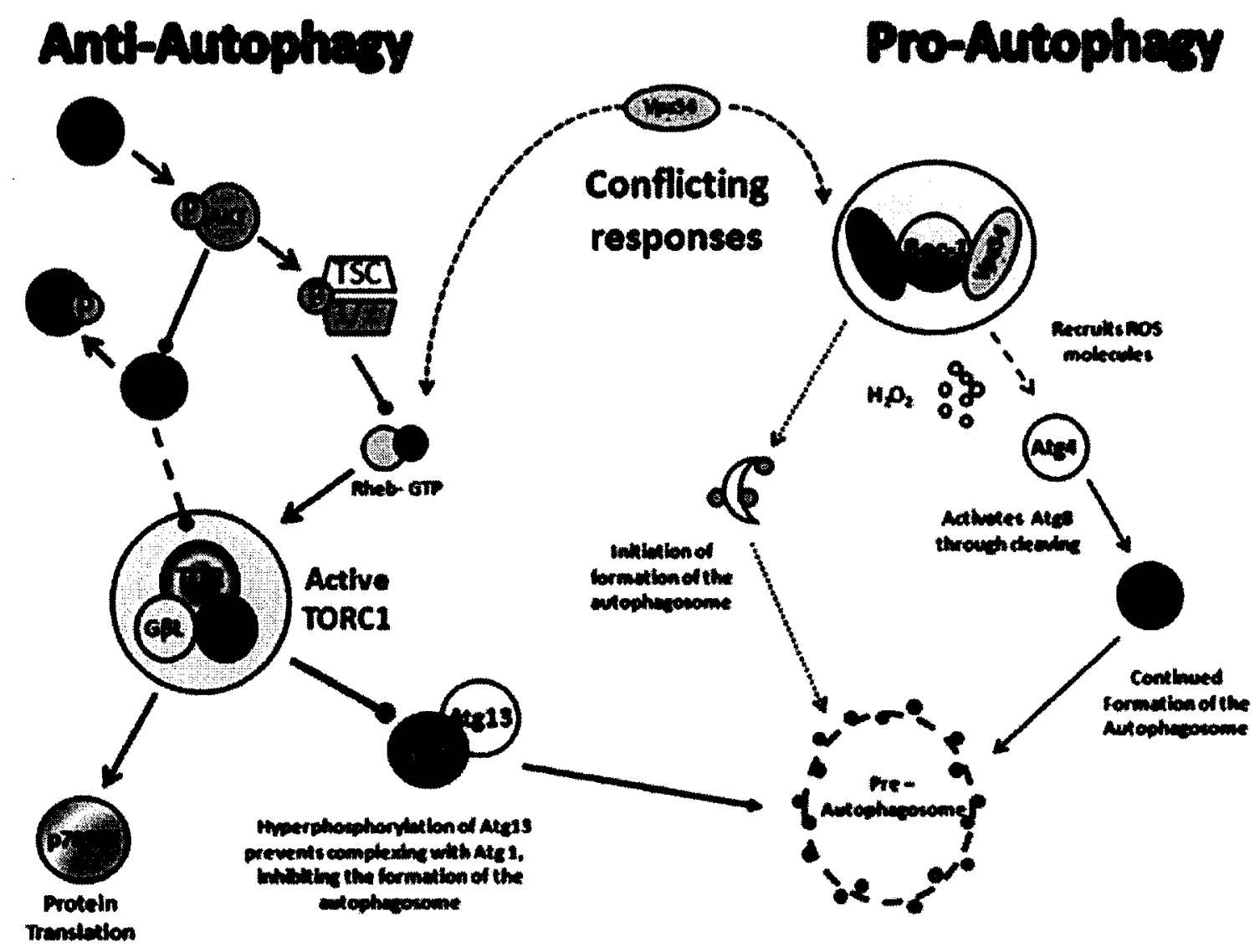

Figure 5.2: Regulation of autophagy by TORC1. The regulation of autophagy is twofold. First through a (direct or indirect) hyperphosphorylation of Atg13, limiting its interaction with Atg1 kinase, and secondly through competition for (nutrient level based) Vps34. 

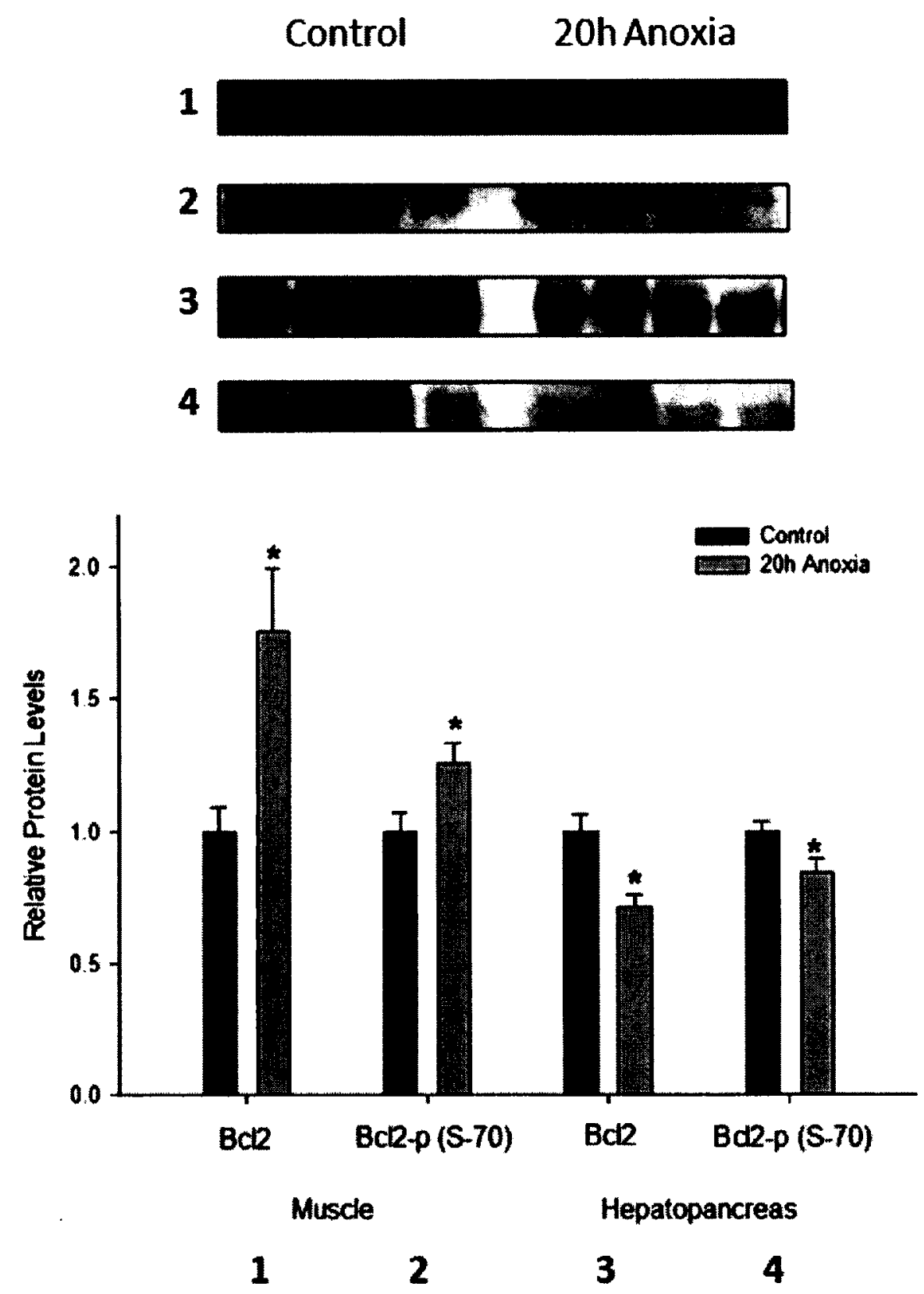

Figure 5.3: Effect of $20 \mathrm{~h}$ anoxic exposure on the Bcl2 protein levels and activation in $O$. virilis tissues.

Changes in $\mathrm{Bcl} 2$ protein levels and phosphorylation of $\mathrm{Bcl} 2$ at serine 70 in 'total' protein extracts of crayfish tissues in response to anoxia, as determined by western immunoblotting.

Data are means \pm SEM, $\mathrm{n}=4$ independent trials for muscle and hepatopancreas. * indicates significantly different from the corresponding control, $P<0.05$. 


\section{Control 20h Anoxia}

1

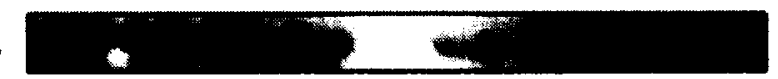

2

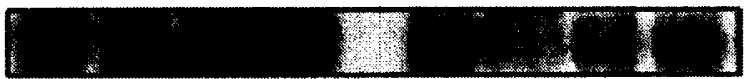

3

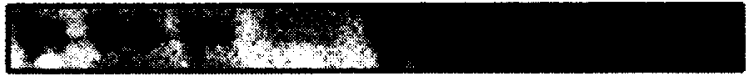

4
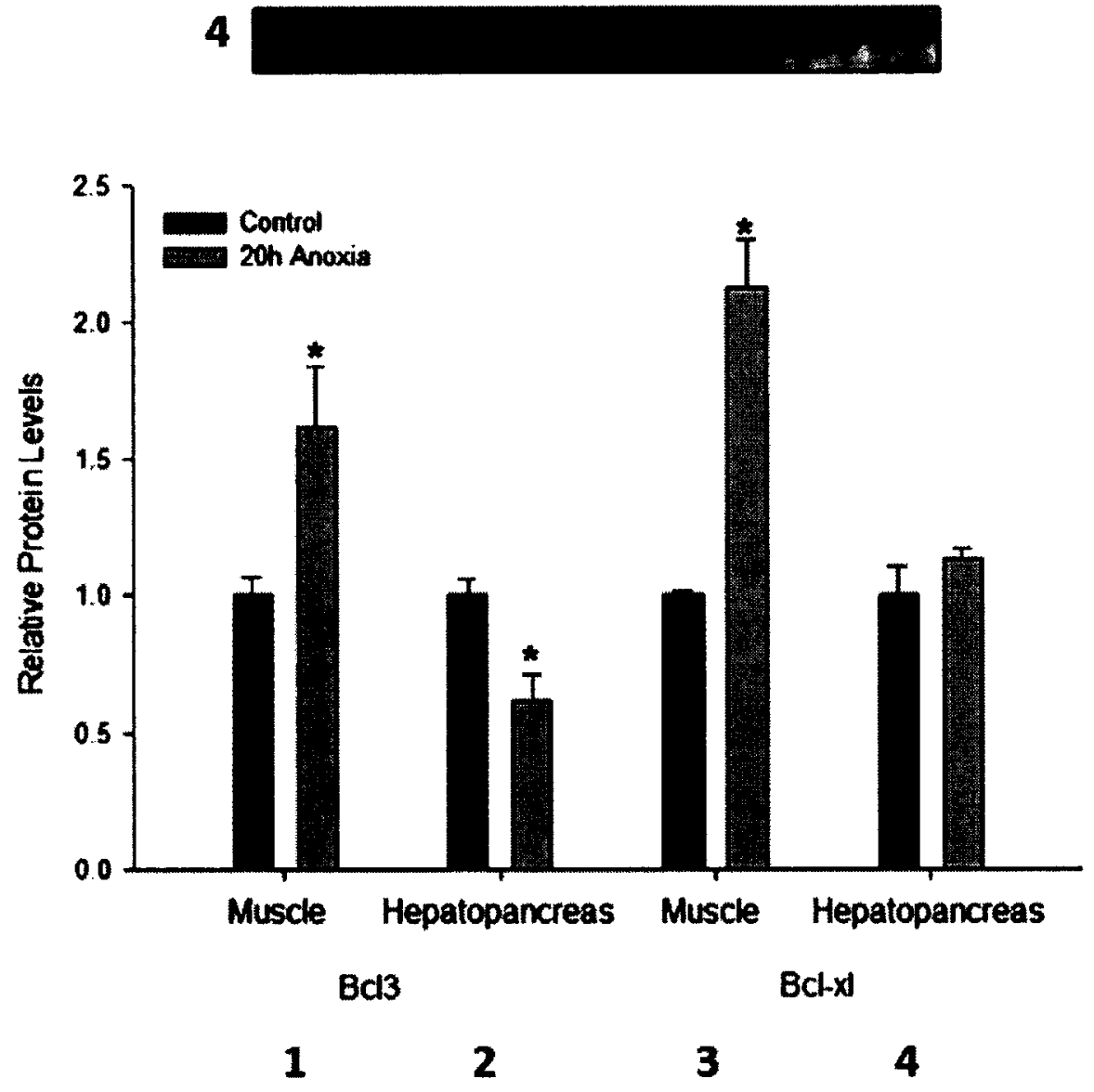

Figure 5.4: Effect of $20 \mathrm{~h}$ anoxic exposure on Bcl3 and Bcl-xl protein levels in $O$. virilis tissues.

Changes in Bcl3 and Bcl-xl protein levels in 'total' protein extracts of crayfish tissues in response to anoxia, as determined by western immunoblotting.

Data are means \pm SEM, $n=4$ independent trials for muscle and hepatopancreas. * indicates significantly different from the corresponding control, $P<0.05$. 


\section{Control 20h Anoxia}

1
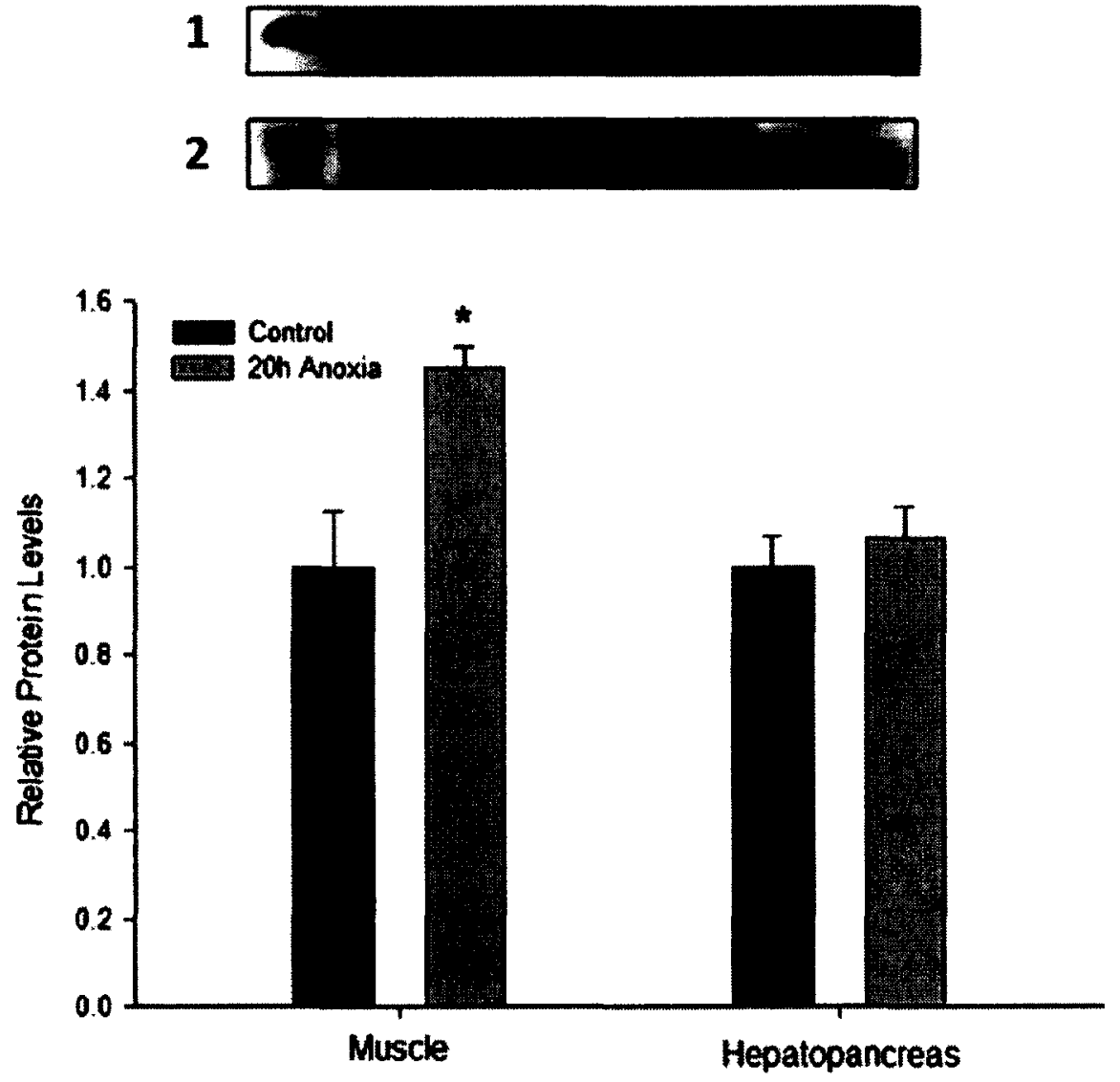

Beclin 1

1

2

Figure 5.5: Effect of $20 \mathrm{~h}$ anoxic exposure on Beclin 1 expression in $O$. virilis tissues. Changes in protein levels of Beclin 1 in 'total' protein fractions of crayfish tissues (tail muscle, left; hepatopancreas, right) in response to anoxia, as determined by western immunoblotting.

Data are means $\pm S E M, n=4$ independent trials for muscle and hepatopancreas. * indicates significantly different from the corresponding control, $P<0.05$. 


\section{Control 20h Anoxia}

1

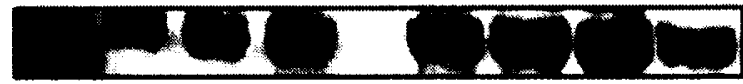

2

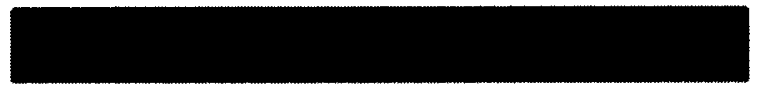

3

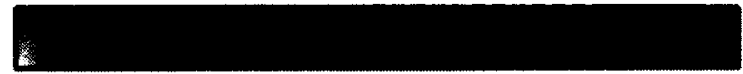

4
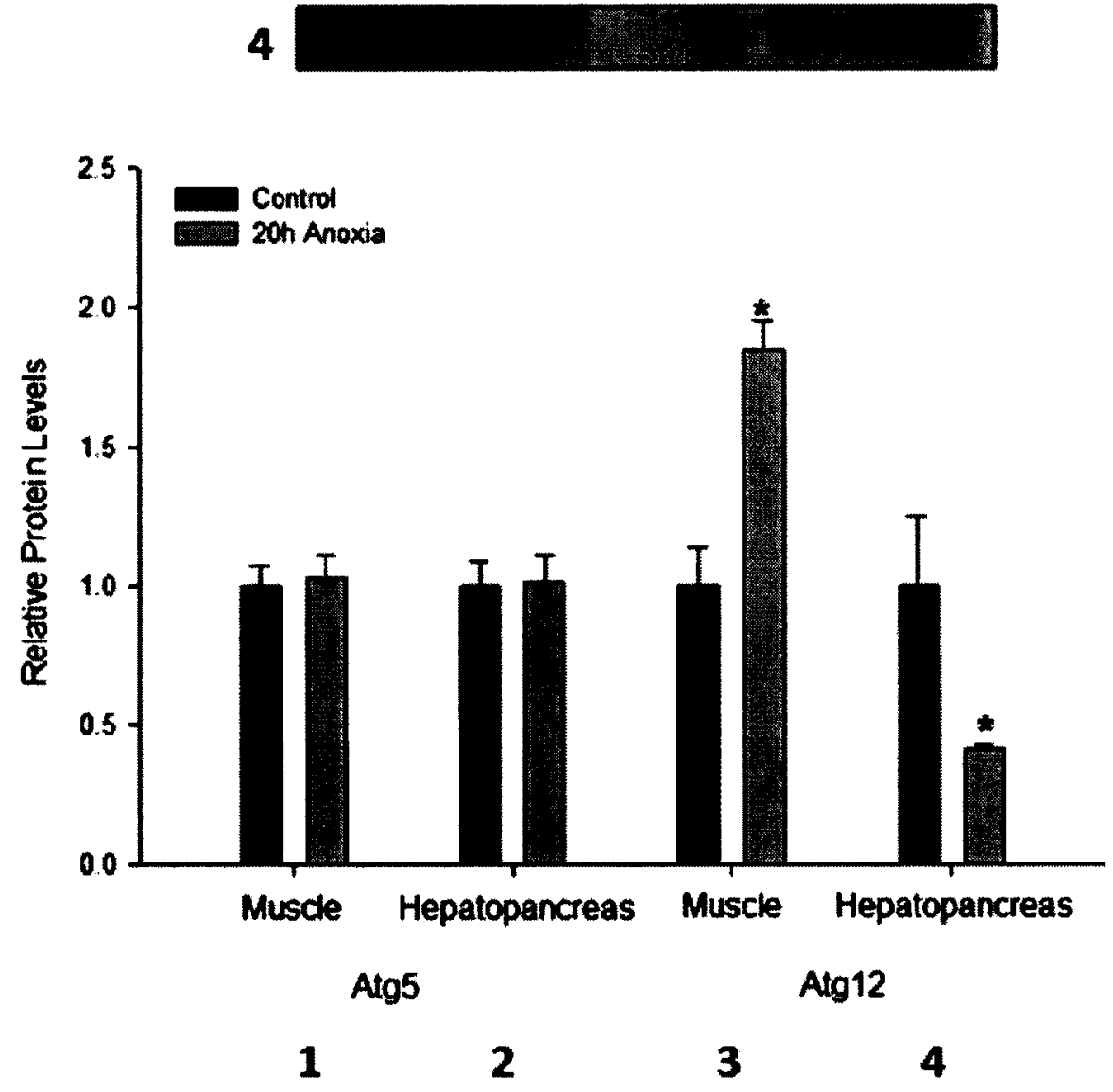

Figure 5.6: Effect of $20 \mathrm{~h}$ anoxic exposure on Atg5 and 12 protein levels in $O$. virilis tissues.

Changes in Atg5 and 12 protein levels in 'total' protein extracts of crayfish tissues in response to anoxia, as determined by western immunoblotting.

Data are means \pm SEM, $n=4$ independent trials for muscle and hepatopancreas. * indicates significantly different from the corresponding control, $P<0.05$. 


\section{Control 20h Anoxia}

1

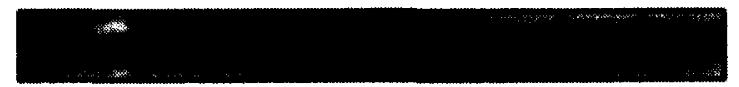

2
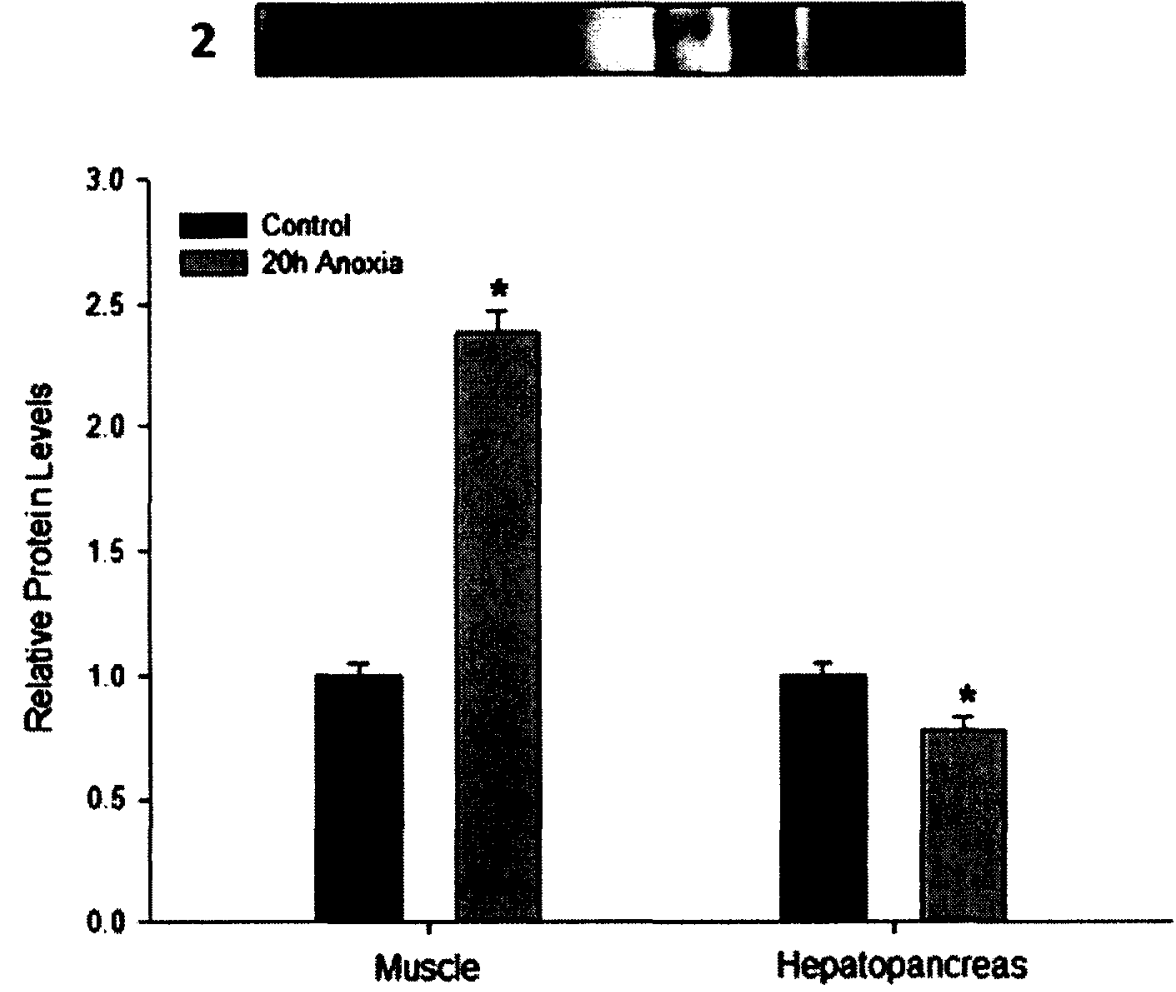

Atg 7

1

Figure 5.7: Effect of $20 \mathrm{~h}$ anoxic exposure on Atg7 expression in $O$. virilis tissues. Changes in protein levels of Atg7 in 'total' protein fractions of crayfish tissues (tail muscle, left; hepatopancreas, right) in response to anoxia, as determined by western immunoblotting.

Data are means \pm SEM, $n=4$ independent trials for muscle and hepatopancreas. * indicates significantly different from the corresponding control, $P<0.05$. 


\section{Control 20h Anoxia}

1

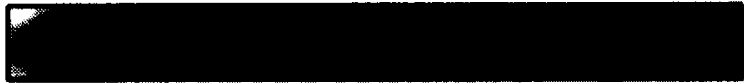

2

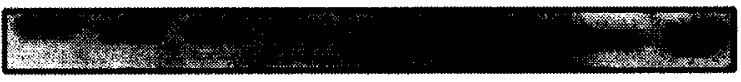

3

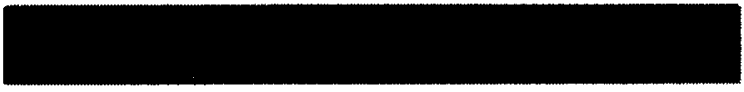

4
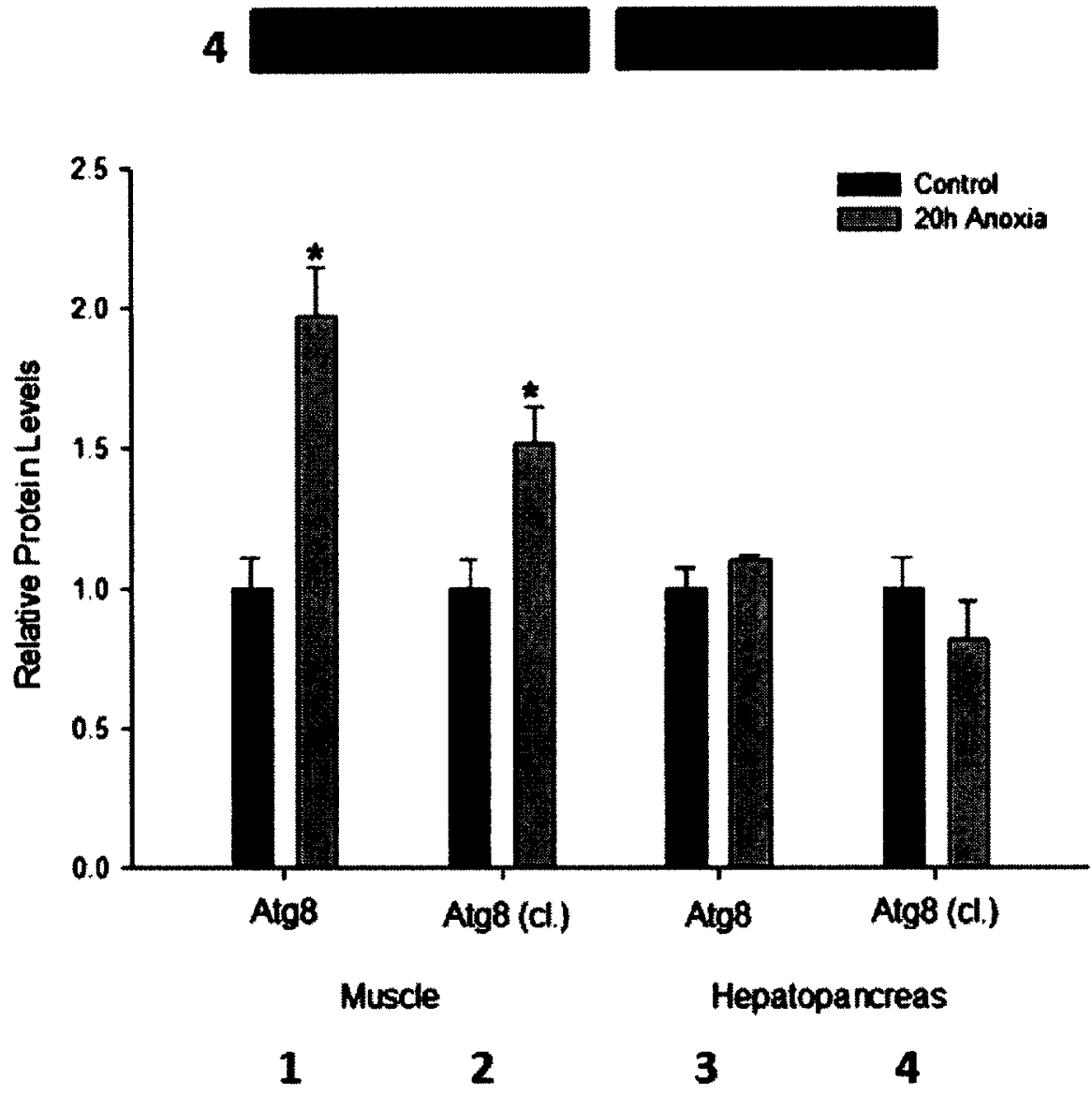

Figure 5.8: Effect of $20 \mathrm{~h}$ anoxic exposure on Atg8 protein levels and activation in $O$. virilis tissues.

Changes in Atg8 protein levels and cleavage of Atg8 in 'total' protein extracts of crayfish tissues in response to anoxia, as determined by western immunoblotting.

Data are means \pm SEM, $n=4$ independent trials for muscle and hepatopancreas. * indicates significantly different from the corresponding control, $P<0.05$. 

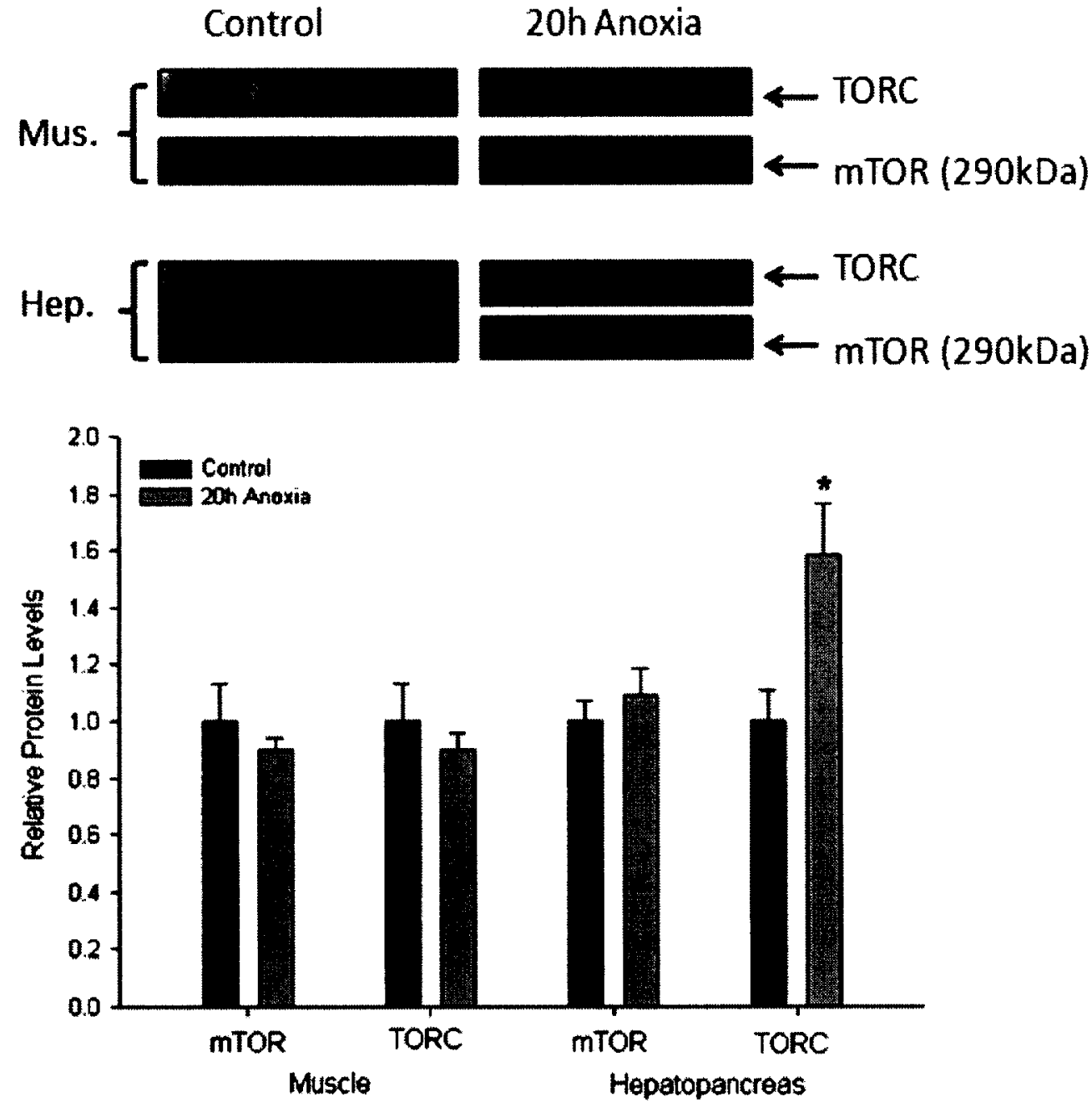

Figure 5.9: Effect of $20 \mathrm{~h}$ anoxic exposure on mTOR expression and TORC incorporation in $O$. virilis tissues.

Changes in mTOR protein levels and TORC in 'total' protein extracts of crayfish tissues in response to anoxia, as determined by immunoprecipitation.

Data are means $\pm S E M, n=4$ independent trials for muscle and hepatopancreas. * indicates significantly different from the corresponding control, $P<0.05$.

Note: In the control condition for hepatopancreas, the mTOR band is located directly underneath the suspected TORC band. Both bands were located at the top of the $6 \%$ acrylamide gel, which exhibited some disfiguration during running (likely due to heating), and as such exhibit some resolution issues and are pictured together. 

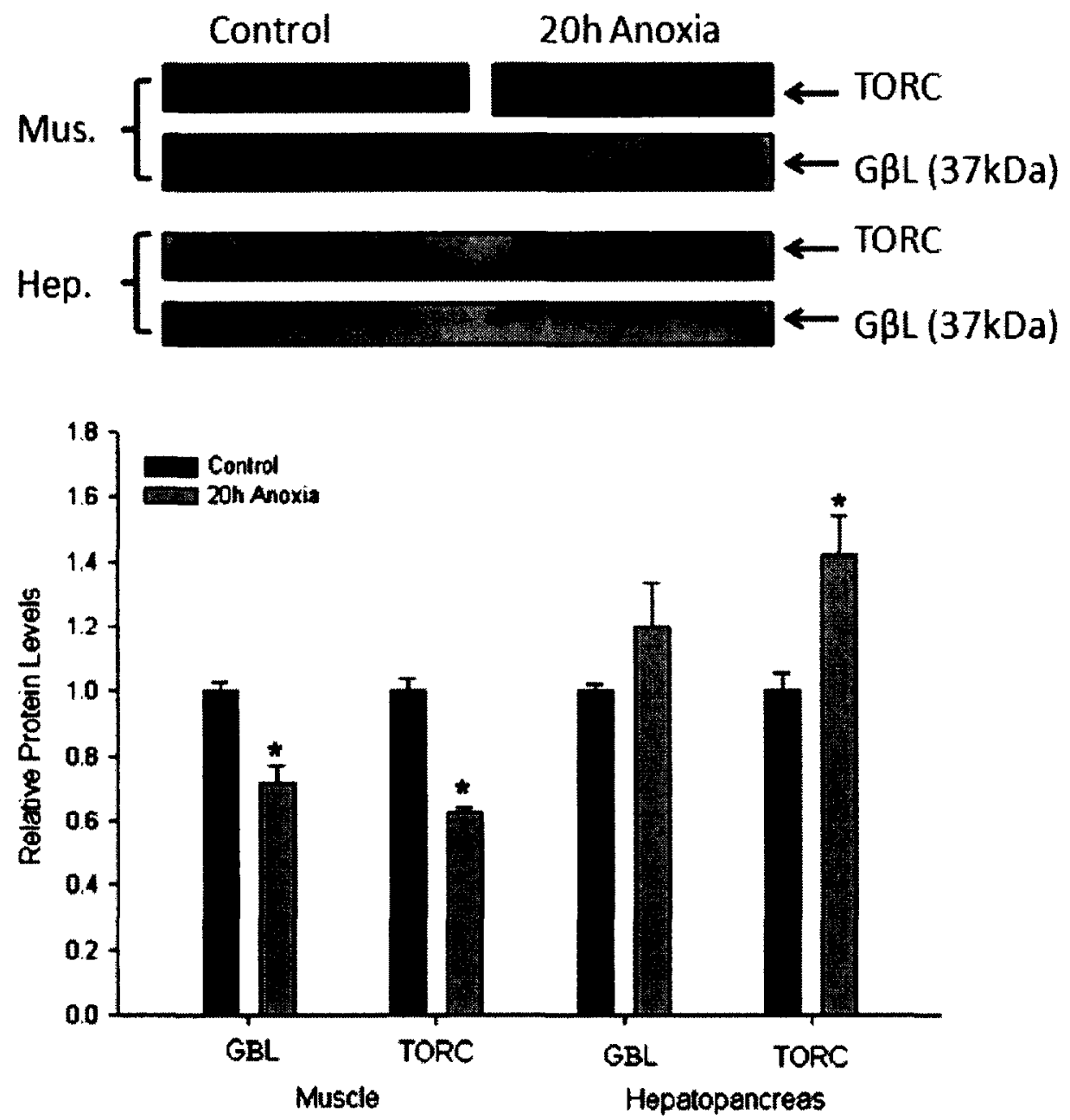

Figure 5.10: Effect of $20 \mathrm{~h}$ anoxic exposure on GBL expression and TORC incorporation in $O$, virilis tissues.

Changes in G $\beta$ L protein levels and TORC in 'total' protein extracts of crayfish tissues in response to anoxia, as determined by immunoprecipitation.

Data are means \pm SEM, $n=4$ independent trials for muscle and hepatopancreas. * indicates significantly different from the corresponding control, $P<0.05$. 


\section{Control 20h Anoxia}

1

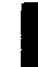

2

3

4
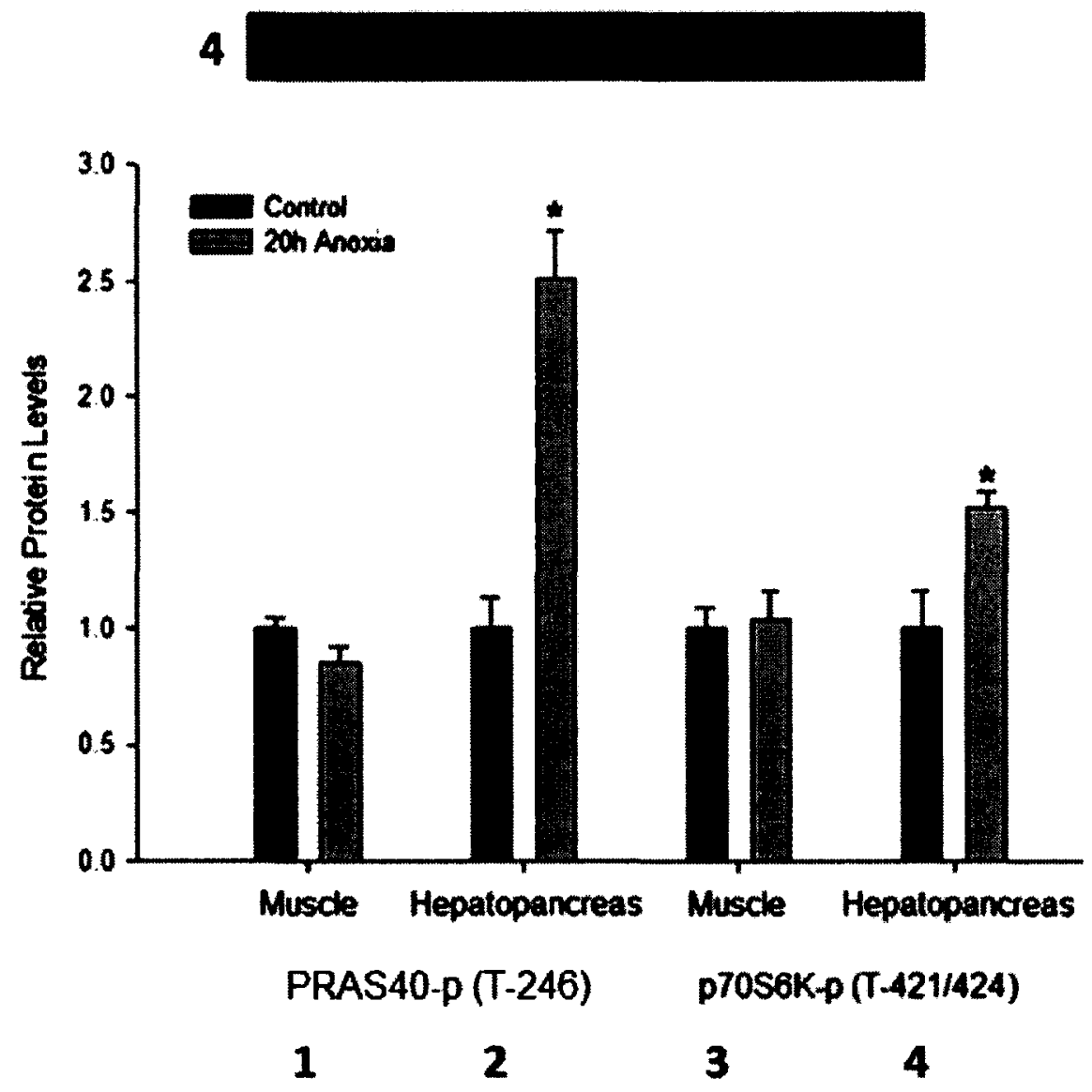

Figure 5.11: Effect of $20 \mathrm{~h}$ anoxic exposure on phosphorylation of PRAS40 and p70S6K in $O$. virilis tissues.

Changes in PRAS40 protein levels and phosphorylation of p70S6K at threonine 421/424 in 'total' protein extracts of crayfish tissues in response to anoxia, as determined by western immunoblotting.

Data are means \pm SEM, $\mathrm{n}=4$ independent trials for muscle and hepatopancreas. * indicates significantly different from the corresponding control, $P<0.05$. 
Table 5.2 Chapter 5 data summary

\begin{tabular}{|c|c|c|c|c|}
\hline & \multicolumn{2}{|c|}{$\begin{array}{l}\text { Protein } \\
\text { (Total) }\end{array}$} & \multicolumn{2}{|c|}{ IP } \\
\hline & $\mathbf{M}$ & H & $\bar{M}$ & $\mathbf{H}$ \\
\hline Bcl 2 & $\uparrow$ & $\downarrow$ & & \\
\hline Bcl 2-p (S70) & $\uparrow$ & $\downarrow$ & & \\
\hline Bcl 3 & $\uparrow$ & $\downarrow$ & & \\
\hline Bcl xl & $\uparrow$ & $\leftrightarrow$ & & \\
\hline Beclin 1 & $\uparrow$ & $\leftrightarrow$ & & \\
\hline $\operatorname{Atg} 5$ & $\leftrightarrow$ & $\leftrightarrow$ & & \\
\hline $\operatorname{Atg} 12$ & $\bar{\uparrow}$ & $\downarrow$ & & \\
\hline $\operatorname{Atg} 7$ & $\uparrow$ & $\downarrow$ & & \\
\hline $\operatorname{Atg} 8$ & $\uparrow$ & $\leftrightarrow$ & & \\
\hline Atg 8 (c) & $\uparrow$ & $\leftrightarrow$ & & \\
\hline mTOR & & & $\leftrightarrow$ & $\leftrightarrow$ \\
\hline mTOR/TORC & & & $\leftrightarrow$ & $\uparrow$ \\
\hline GßL & & & $\downarrow$ & $\leftrightarrow$ \\
\hline GRL/TORC & & & $\downarrow$ & $\uparrow$ \\
\hline $\begin{array}{l}\text { PRAS40-p } \\
\text { (T246) }\end{array}$ & $\leftrightarrow$ & $\uparrow$ & & \\
\hline $\begin{array}{l}\text { p70S6K-p } \\
\text { (T421/424) }\end{array}$ & $\leftrightarrow$ & $\uparrow$ & & \\
\hline
\end{tabular}

Note: Changes indicate statistical significance $(P<0.05)$; Western Blotting $(n \geq 4)$, IP $(n=4)$

Arrows indicate changes or level profiles, in response to anoxia 


\section{Chapter 6 \\ Regulation of the cell cycle during oxygen deprivation by the anoxia resistant crayfish, $O$. virilis}




\subsection{Introduction}

The cell cycle controls rates of cellular proliferation, and indeed aging. It is a multi-step process which is tightly regulated by many post-translational modifications, primarily reversible phosphorylation (FIG. 6.1). There are four stages of the cell cycle, beginning with gap phase $1\left(G_{1}\right)$, transitioning into the DNA synthesis phase $(S)$. The $G_{1} / S$ barrier is one of the major regulatory steps, with many of the crucial pieces of cell cycle machinery contributing to transition through the barrier or attenuation of the cell cycle at this point. The $S$ phase is followed by the second gap phase $\left(G_{2}\right)$, which is followed by mitosis $(M)$. The $\mathrm{G}_{2} / \mathrm{M}$ barrier is the second major regulation point of the cell cycle. Following completion of the cell cycle, the process can continue, or move into a non-dividing quiescence $\left(G_{0}\right)$, which is a temporary attenuation leaving the cell cycle in the $G_{1}$ stage.

The primary regulators of the cell cycle are complexes of cyclins and cyclin dependent kinases (CDKs) which mediate transitions into each of the four stages. Cyclins have no activity themselves, but are required by the CDKs, prior to complex phosphorylation, which stimulates the active site of the kinase (Obaya and Sedivy, 2002). Through $\mathrm{G}_{1}$ into $\mathrm{S}$ phase, cyclin $\mathrm{D} / \mathrm{CDK} 4 / 6$ and cyclin $\mathrm{E} / \mathrm{CDK} 2$ complexes are necessary. Through the $S$ phase and into the $G_{2}$ phase, cyclin $A / C D K 2$ complex activity is necessary, followed by cyclin A/B/CDK1 activity to enter mitosis (Obaya and Sedivy, 2002). This progression of cyclin/CDK activity allows specific regulation through each barrier, and target phosphorylation of key cell cycle proteins. Expression levels of cyclins at specific junctures (i.e. $D$ and $E$ at $G_{1}, A$ and $B$ at $G_{2}$ ) are indicative of how the cell cycle is progressing; indeed over expression of cyclins result in significantly reduced gap phases (Ohtsubo e al., 1995). Cyclin production and activity is, as such, a closely 
regulated procedure. For example, cyclin $\mathrm{D}$, which is critical for $\mathrm{S}$ phase transition aside from specific cell cycle inhibitors, is subject to inhibition through phosphorylation (GSK3; Diehl et al., 1998) and transcriptional repression (p53; Rocha et al., 2003 and via CMYC inhibition; Ladu et al., 2008).

Control of cell cycle barriers

Indeed, probably the most important regulatory barrier, the $G_{1} / S$ transition, is partially controlled by cyclin D expression. At this junction, the critical point of control is the activity of the retinoblastoma $(\mathrm{Rb})$ tumor suppressors. The $\mathrm{Rb}$ proteins are a family of proteins act to affect E2F transcription factor activity. The E2F transcription factors can be divided into pro-cell cycle progression ("activating") and anti-cell cycle progression ("repressor") sub groups (Trimarchi and Lees, 2002); E2F-1 an example of an activating member, E2F-4 an example of a repressive member. Members of the $\mathrm{Rb}$ family interact specifically with either the activating E2Fs (Rb/p105) or repressive E2Fs (Rb2/p130), in the case of the former to prevent transcription of critical cell cycle targets, and in the case of the latter to compete with 'activating' E2F binding sites (Trimarchi and Lees, 2002). Hence active $R b$ is a target of cell cycle progression. Through phosphorylation of $R b$, via cyclin D/CDK4/6 (and secondarily, cyclin E/CDK2), the protein is inactivated and targets such as cyclin A and E can be transcribed (Korenack and Brehm, 2005).

In the $\mathrm{S}$ phase, increased cyclin $\mathrm{A}$ expression results in increased cyclin $\mathrm{A} / \mathrm{CDK} 2$ activity, which is crucial for activating DNA replication components (Cardoso et al., 1993). At the $\mathrm{G}_{2}$ Stage, cyclin/CDK complexes, specifically CDK1 (aka Cdc2) in complex with cyclin A and B, form maturation promoting factors (MPFs). MPFs control transition from $\mathrm{G}_{2}$ into mitosis by phosphorylating numerous factors including chromatin 
condensers (condensins), microtubule associated proteins and histones (Bellé et al., 1990).

Control of cyclin/CDK complexes

As the primary effectors of the cell cycle and its progression through each of its major subsections, the cyclin/CDK complexes are the targets of regulation through various systems. Cell cycle inhibitors typically form two classes, the cyclin inhibiting proteins ( $\mathrm{Cip}$ ) and the kinase inhibiting proteins (Kip), but within these classes there are further divisions such as the 'inhibitors of CDK4' (INK4) family (Sherr and Roberts, 1999). Within the INK4 family there are members p15, p16, p18 and p19. By binding CDK4, the INK4 proteins reduce cyclin $D$ affinity, affecting $G_{1} / S$ transition. Similarly, the p21 Cip and p27 Kip inhibit CDK2 complexes by binding cyclins and CDK2 respectively (Vidal and Koff, 2000). These cell cycle inhibitors are regulated by numerous major cellular components that contribute to either cellular survival or cell death ('tumor suppressing') signaling. For example, the pro-survival/proliferation Akt signaling (via insulin/IGF signaling) negatively regulates p21 and p27 production through forkhead box transcription factor suppression (Osaki et al., 2004). Similarly c-Myc, a major cellular transcriptional regulator, is involved in cell cycle progression. Through inhibition of $\mathrm{p} 27, \mathrm{p} 21$ and the $\mathrm{p} 57 \mathrm{Kip}$, along with the induction of major pro-cell cycle genes such as cdc25A, c-Myc is able to increase cyclin/CDK complex activity which in turn increases Rb phosphorylation and S phase progression (Amati et al., 1998). Phospho-control of the cell cycle

In the same way that phospho-control of $R b$ is a crucial step to $G_{1} / S$ transition, control of the phospho state of the cyclin/CDK complexes is critical (FIG 6.1). To be 
active, cyclin/CDK complexes must be phosphorylated on CDK threonine residues, proximal to their active site (the 'T-loop' region) by CDK-activating kinase (CAK) (Hannon et al., 1994). This phosphorylation can be reversed by the kinase associated phosphatase (KAP), which interacts with cdc2 (CDK1) and CDK2, reducing amongst other effects, S-phase progression (Lee et al., 2000). Cyclin/CDK complexes, however, can also be inhibited by phosphorylation. The Wee family of kinases (including Weel, Wee2 and Myt1) can phosphorylate both threonine and tyrosine residues of CDKs (Berthet and Kaldis, 2007). Wee1, for example is capable of phosphorylating CDK2 (Wroble et al., 2007), and cdc2 (Heald et al., 1993) on tyrosine residues, inhibiting both progression of the cell cycle through the S phase or mitosis. In the case of MPF phospho control, both phosphorylation and dephosphorylation events are needed prior to full activation. Along with activating phosphorylation, cdc25C critically dephosphorylates cdc2 (at sites phosphorylated by Wee kinases) (Heald et al., 1993), allowing activity. Cdc25c itself can be inhibited by, amongst others, checkpoint kinase (Chks) (Reinhardt and Yaffe, 2009) and PKA (Duckworth et al., 2002) phosphorylation.

The checkpoint kinase pathway is intrinsically signaled as part of a quality control mechanism; error-checking in DNA replication. DNA damage, either by oxidative of genotoxic stress, can trigger a conserved signal transduction through the ATM/ATR kinases, which work upstream of Chk1 and 2 to phosphorylate and activate them (Choudhury et al., 2007). Activated Chk1 and 2 are able to phosphorylate cdc25A (which maintains CDK2 (complexed with cyclin A or E)), inhibiting it, and also increasing ubiquitination and subsequent proteasomal degradation (Choudhury et al., 2007). 
Similarly, Chk1, as mentioned above, is capable of phosphorylating $\operatorname{cdc} 25 \mathrm{C}$, halting the progression of mitosis.

In this chapter, the cell cycle and its regulators was studied to provide an overview of its progression, in response to anoxia in the freshwater crayfish, $O$. virilis. The current status of cell cycle regulation in response to oxygen deprivation is under developed. Cases of study in embryonic development, typically in response to hypoxia, of certain animal models (Padilla and Roth, 2001; Clegg, 1997) have suggested that cell cycle arrest may be a response to the reduced energy status of the cell. Indeed, the cell cycle of the nematode $C$. elegans, prior to its third larval stage, is completely attenuated and enters the quiescent stage (see Appendix A and B.6) (Clayton et al., 2008).

In $O$. virilis, the overlying trend appears to be cell cycle progression throughout the anoxic period. The combined profile of nuclear profiling of target cyclins, along with $\mathrm{Rb}$ phosphorylation and nuclear E2F presence would suggest an active cell cycle. Despite this, E2F DNA binding levels remain largely unchanged, p16 (although not uniformly) and Chk phosphorylation levels increase under anoxia; indicating regulation of the level of cell cycle progression. Hence there may be a reduction in cell cycle activity that does not attain the level of quiescence.

\subsection{Materials and Methods}

6.2.1. Animals

Crayfish were treated and tissue samples were collected as described in Chapter 2.

\subsubsection{RNA extraction, $c D N A$ synthesis, primer design and RT-PCR}

Total RNA was isolated from crayfish tissues and cDNA was reverse transcribed and serially diluted as described in Chapter 2. Forward and reverse primers for $R b$ were designed from conserved regions in these genes based on DNA alignment of the 
sequences from both vertebrate and invertebrate species. Following initial work using consensus primers, perfect (crayfish-specific) primers were then designed from crayfish sequences and used for quantification of transcript levels. Primers for the control gene, $\alpha-$ tubulin, were as in Chapter 2. Primers and cDNA product sizes are listed in Table 6.1.

PCR reactions were composed as described in Chapter 2. The PCR program was as follows: $7 \mathrm{~min}$ at $94^{\circ} \mathrm{C}$ followed by 35 cycles of $1 \mathrm{~min}$ at $94^{\circ} \mathrm{C}, 1 \mathrm{~min}$ at a predetermined annealing temperature $\left(58^{\circ} \mathrm{C}\right)$, and $1.5 \mathrm{~min}$ at $72^{\circ} \mathrm{C}$. The final step was $72^{\circ} \mathrm{C}$ for $10 \mathrm{~min} . \mathrm{PCR}$ products were separated on a $1 \%$ agarose gel stained with ethidium bromide, visualized using the ChemiGenius imaging system (Syngene, Frederick, MD, USA) under UV light and quantified using the GeneTools program. The bands from the most dilute cDNA sample that gave visible product were used for quantification to ensure that the products had not reached amplification saturation. PCR products were sequenced by DNA Landmarks (St.-Jean-sur-Richelieu, QC) and sequences were verified as encoding the correct genes using the program BLASTN (http://www.ncbi.nlm.nih.gov/blast) at the NIH.

\subsubsection{Protein extracts and western blotting}

Protein extracts were prepared from tissue samples of control and $20 \mathrm{~h}$ anoxiaexposed crayfish. Equal amounts of protein $(20-30 \mu \mathrm{g})$ were loaded into each lane of $10 \%$ SDS polyacrylamide gels, electrophoresed and then electroblotted onto polyvinylidene difluoride (PVDF) membranes as described in Chapter 2. After the transfer of proteins, membranes were blocked for $15 \mathrm{~min}$ in Tris buffered saline containing Tween-20 (TBST: $20 \mathrm{mM}$ Tris base, $140 \mathrm{mM} \mathrm{NaCl}, 0.1 \% \mathrm{v} / \mathrm{v}$ Tween-20) with $2.5 \%$ non-fat dried milk added. Blots were probed overnight with primary antibodies 
(listed in TABLE 6.2). All primary antibodies were diluted 1:1000 in TBST. After incubation, blots were washed several times with TBST and then incubated at RT for $1.5 \mathrm{~h}$ with HRP-linked goat anti-rabbit IgG (Cell Signaling, diluted 1:2000 in TBST). Immunoblots were developed using enhanced chemiluminescence.

\subsubsection{Preparation of nuclear extracts and DNA- binding assay}

Nuclear extracts for DNA-binding assay were prepared as described in chapter 2. Aliquots containing equal amounts of protein from each sample ( $20 \mu \mathrm{g} / \mathrm{well})$ were then used to assess the amount of binding by E2F-1 and 4 to its DNA binding element as described in Chapter 2. The sequence of the biotin-conjugated probe, for both of the E2F members, was 5'- Biotin-ATTTAAGTTTCGCGCCCTTTCTCAA-3', whereas the complementary sequence was 5'- TTGAGAAAGGGCGCGAAACTT- 3'. Aliquots of nuclear extracts containing $20 \mu \mathrm{g}$ of protein were combined with $50 \mu \mathrm{L}$ of $1 \times$ protein binding buffer [10 mM Hepes, pH 7.9, $50 \mathrm{mM} \mathrm{KCl}, 0.5 \mathrm{mM}$ EDTA, $3 \mathrm{mM} \mathrm{MgCl}_{2}, 10 \%$ $\mathrm{v} / \mathrm{v}$ glycerol, $0.5 \mathrm{mg} / \mathrm{mL}$ BSA, $0.05 \% \mathrm{NP}-40,1 \mu \mathrm{g}$ salmon sperm DNA (BioShop), 0.5 mM DTT, and $\mathrm{NaCl}$ (variable amount was used based on the volume of nuclear extract added, final concentration was always $40 \mathrm{mM}$ )]. The plate was incubated at RT with mild agitation for $75 \mathrm{~min}$ and then washed 4 times with wash buffer. Aliquots of $60 \mu \mathrm{L}$ of the primary antibody for E2F-1 and 4 (both Cell Signaling) were diluted 1:250 in PBS, then added to the wells. The plate was incubated for $3 \mathrm{~h}$. The remainder of the procedure was as described in Chapter 2.

\subsubsection{Electromobility Shift Assay}

In order to confirm probe efficiency for the DNA-binding activity ELISA, an electromobility shift assay was performed to visualize probe binding. First, biotinylated 
probe from the DNA binding activity assay $(250 \mathrm{pmol} / \mu \mathrm{l})$ was diluted to $10 \mathrm{pmol} / \mu 1$. Aliquots of $4 \mu \mathrm{l}(8 \mu \mathrm{g})$ of nuclear extract were mixed with $1 \mu \mathrm{l}$ DEPC treated $\mathrm{H}_{2} \mathrm{O}$ and 4 $\mu$ EM SA master mix; 5X EMSA binding buffer contained [50mM Tris $\mathrm{HCl}, \mathrm{pH} 7.8$, $250 \mathrm{mM} \mathrm{NaCl}, 5 \mathrm{mM}$ EDTA and $25 \%$ glycerol combined with $1 \mu \mathrm{g} / \mu 1$ Poly d(I-C) in a 1:1 v:v ratio. Samples were vortexed and incubated at RT for $5 \mathrm{~min}$ and then $1 \mu \mathrm{l} \mathrm{TF}$ probe was added and the mixture was vortexed again before placing in the thermocycler for $30 \mathrm{~min}$ at $15^{\circ} \mathrm{C}$. The positive control was biotinylated E2F Probe (Panomics EMSA Kit 7104) tested with turtle (Trachemys scripta elegans) liver nuclear extract, in the same mix as above. Negative controls for no protein and no probe were also run, with adjustments to water volume as needed to keep total volume at $9 \mu$.

Following incubation, samples were mixed with $1 \mu \mathrm{l}$ 6x DNA Loading dye (BioShop) prior to loading on a $6 \%$ non-denaturing polyacrylamide gel [ $1 \mathrm{ml} 10 \mathrm{X}$ TBE (chilled), $4 \mathrm{ml} 30 \%$ bis-acrylamide, $625 \mu \mathrm{l} 80 \%$ glycerol, $14.375 \mathrm{ml}$ deionized sterile $\mathrm{H}_{2} \mathrm{O}$, $300 \mu 110 \%$ APS and $20 \mu \mathrm{l}$ TEMED - total $2 \times 10 \mathrm{ml} / \mathrm{gel})$. The gels were cast as in the Western blotting procedure (above). Gels were pre- run in chilled $0.5 \mathrm{x}$ TBE for $10 \mathrm{~min}$ at $120 \mathrm{~V}$. Samples were then loaded into the gels and run at $120 \mathrm{~V}$ for $70 \mathrm{~min}$ at $4^{\circ} \mathrm{C}$ (fridge). Following electrophoresis, proteins were transferred to Pall Biodyne B nylon membranes. Membranes were pre-soaked in $0.5 \mathrm{x}$ TBE and then gels were sandwiched to membranes as described for the Western blotting protocol. Proteins were transferred in $0.5 \mathrm{x}$ TBE (chilled) for $30 \mathrm{~min}$ at $300 \mathrm{~mA}$ at $4^{\circ} \mathrm{C}$. Once transfer was complete, membranes were stored between dry filter paper, and the oligos were fixed to membranes in an oven $\left(80^{\circ} \mathrm{C}\right)$ for $1 \mathrm{~h}$. Following this, membranes were washed on a rocking platform with $5 \mathrm{ml}$ (per membrane) of 1x EMSA Blocking Buffer (diluted from Panomics CS7535) for 30 
min at RT. The blocking buffer was decanted and to it $5 \mu \mathrm{V} / \mathrm{ml}$ Streptavidin-HRP mixture (BioShop) was added. This mixture was reapplied to the membrane and left to incubate on a rocking platform for a further $30 \mathrm{~min}$ at RT. Finally, membranes were washed 3 times, consecutively, with $1 \mathrm{x}$ wash buffer (diluted from Panomics 7537). The membranes were then exposed using ECL solutions as in the Western blotting protocol.

\subsubsection{Data Analysis}

All data were analyzed as described in Chapter 2. 
Table 6.1 List of Primers used in Chapter 6

\begin{tabular}{|l|l|c|c|}
\hline \multicolumn{1}{|c|}{ Gene/primer } & Primer sequence (5'-3') & $\begin{array}{c}\text { Annealing } \\
\text { temp. } \\
\left({ }^{\circ} \mathbf{C}\right)\end{array}$ & $\begin{array}{c}\text { Expected } \\
\text { product size } \\
\text { (bp) }\end{array}$ \\
\hline$R b$ perfect forward & ACGTGAACAGTTCTACCTGG & & \\
\cline { 1 - 2 }$R b$ perfect reverse & CTCCTTCCAAGTCACAGTGC & 55 & $\sim 160$ \\
\hline
\end{tabular}

Table 6.2 List of primary antibodies used in Chapter 6

\begin{tabular}{|l|l|l|}
\hline \multicolumn{1}{|c|}{$\begin{array}{c}\text { Primary } \\
\text { Antibody }\end{array}$} & \multicolumn{1}{c|}{ Company } & \multicolumn{1}{c|}{ Catalogue \# } \\
\hline$C D K 2$ & Cell Signaling (Danvers, MA, USA) & $\# 2546$ \\
\hline$C D K 4$ & Cell Signaling (Danvers, MA, USA) & $\# 2906$ \\
\hline$C D K 6$ & Cell Signaling (Danvers, MA, USA) & $\# 3136$ \\
\hline$C y c l i n A$ & Cell Signaling (Danvers, MA, USA) & $\# 4656$ \\
\hline$C y c l i n B 1$ & Cell Signaling (Danvers, MA, USA) & $\# 4135$ \\
\hline$C y c l i n D I$ & Cell Signaling (Danvers, MA, USA) & $\# 2978$ \\
\hline$C y c l i n E$ & Cell Signaling (Danvers, MA, USA) & $\# 4129$ \\
\hline$R b-p(S 780)$ & Cell Signaling (Danvers, MA, USA) & $\# 9307$ \\
\hline$E 2 F-1$ & Cell Signaling (Danvers, MA, USA) & $\# 3742$ \\
\hline$E 2 F-4$ & Abcam (Cambridge, MA, USA) & ab11951 \\
\hline$C h k 1-p(S 317)$ & Cell Signaling (Danvers, MA, USA) & $\# 2344$ \\
\hline$C h k 2-p(S 19)$ & Cell Signaling (Danvers, MA, USA) & $\# 2666$ \\
\hline$C d c 25 C$ & Cell Signaling (Danvers, MA, USA) & $\# 4688$ \\
\hline$P 15 / 16$ & $\begin{array}{l}\text { Santa Cruz Biotechnology (Santa Cruz, CA, } \\
\text { USA) }\end{array}$ & sc-28260 \\
\hline$P 16-p(S 152)$ & Epitomics (Burlingame, CA, USA) & T3748 \\
\hline$P 21-p(T 145)$ & $\begin{array}{l}\text { Santa Cruz Biotechnology (Santa Cruz, CA, } \\
\text { USA) }\end{array}$ & sc-20220-R \\
\hline$P 27$ & Genscript (Piscataway, NJ, USA) & $\# 3688$ \\
\hline
\end{tabular}




\subsection{Results}

\subsection{Protein expression of cyclin dependent kinases in response to anoxia}

The cyclin dependent kinases moderate cell cycle progression through the $G_{1} / S$ and $G_{2} / M$ barriers. CDK 2 associates with cyclin $E$ (during the $G_{1}$ phase) and cyclin A (during the $S$ and $G_{2}$ phases). CDK4 and 6 both associate with cyclin $D 1$ during the $G_{1}$ phase. The primary function of cyclin/CDK complexes is to promote transcription of pro-cell cycle targets through phosphorylation and repression of the retinoblastoma protein. Changes in protein levels of CDK 2 and 4 were assessed, in response to anoxia, using immunoblotting (FIG 6.2). Polyclonal CDK2 and CDK4 antibodies cross reacted with bands of $\sim 33$ and $30 \mathrm{kDa}$, which are their expected sizes, respectively. In the tail muscle, under anoxia, both CDK2 and 4 protein levels increased significantly; $1.9 \pm 0.1$ fold and $2.2 \pm$ fold respectively (both $\mathrm{P}<0.05$ ). In the hepatopancreas, $\mathrm{CDK} 4$ levels rise $(1.5 \pm$ 0.1 fold; $\mathrm{P}<0.05)$, as in the tail muscle, but CDK2 protein levels decreased $(20 \pm 7 \%$; $<0.05)$ under anoxia.

Active CDK6 complexes are primarily located in the nucleus and changes in the protein levels of CDK6 in 'cytoplasmic' and 'nuclear' fractions, in response to anoxia were assessed using immunoblotting (FIG.6.3). Polyclonal CDK6 antibodies crossreacted with protein bands of $\sim 36 \mathrm{kDa}$, which is their expected size. In the tail muscle, in the cytoplasmic fraction, protein levels decreased significantly $(45 \pm 2 \% ; \mathrm{P}<0.05)$, while there was a substantial increase $(2.5 \pm 0.2$ fold; $P<0.05)$ in nuclear protein levels, under anoxia. In the hepatopancreas, CDK6 protein levels increased in both cytoplasmic and nuclear fractions, significantly under anoxia; $1.9 \pm 0.1$ and $1.5 \pm 0.09$ fold, respectively (both $\mathrm{P}<0.05$ ).

\subsubsection{Protein expression, and cellular localization of cyclins in response to anoxia}


Cyclins in complex with cyclin dependent kinases form active kinase units that phosphorylate anti-cell cycle components to promote cell cycle progression. Cyclins D and $E$ bind to CDK 2 and $4 / 6$ respectively to regulate the $G_{1} / S$ barrier. Cyclins $A$ and $B$, on the other hand, are primarily associated with progression into mitosis across the $G_{2} / M$ barrier. Cyclin $\mathrm{A}$ associates with $\mathrm{CDK} 2$ in order to enter into mitosis. Cyclin $\mathrm{B}$ associates with CDK1 (cdc2) to promote mitosis. Cyclin CDK complexes often have nuclear targets so cellular localization is crucial to assessing their activity pattern. Cyclin D, E, A and B protein levels were measured in cytoplasmic and nuclear fractions, in response to anoxia, using immunoblotting. Polyclonal cyclin D, E, A and B antibodies cross reacted with protein bands of $\sim 36,50,55$ and $60 \mathrm{kDa}$, respectively, which are their expected sizes.

Cyclin D (FIG. 6.4) protein levels in the tail muscle showed no changes with anoxia in the cytoplasmic fraction, but decreased in the nucleus $(20 \pm 5 \% ; \mathrm{P}<0.05)$. In the hepatopancreas, however, cyclin D protein levels were seen to increase significantly under anoxic conditions in both cytoplasmic and nuclear fractions; $1.9 \pm 0.2$ fold and 1.4 \pm 0.1 fold respectively (both $\mathrm{P}<0.05$ ).

Cyclin E (FIG 6.5) protein levels, unlike cyclin D, showed no change in either tissue (or indeed either fraction) in response to anoxia.

Cyclin A (FIG 6.6) protein levels increased in the cytoplasmic fractions in both the tail muscle and hepatopancreas, under anoxia; $2.4 \pm 0.2$ fold and $1.5 \pm 0.1$ fold, respectively (both $\mathrm{P}<0.05$ ). In contrast, cyclin A protein levels remained static in nuclear fractions of both tissues, under anoxic conditions.

Cyclin B (FIG 6.7) protein levels exhibited significant increases with anoxia in the crayfish tissues, with the exception of the cytoplasmic fraction of the tail muscle in 
which cyclin B remained level. In the nuclear fractions of both tail muscle and hepatopancreas, cyclin B levels increased to $1.9 \pm 0.1$ fold and $1.3 \pm 0.09$ fold respectively (both $\mathrm{P}<0.05$ ). Cyclin $\mathrm{B}$ protein levels also increased in the cytoplasmic fraction of the hepatopancreas $(1.8 \pm 0.1$ fold; $\mathrm{P}<0.05)$, under anoxia.

\subsection{3. cDNA cloning of crayfish $R b$}

A PCR product of $159 \mathrm{bp}$ was retrieved using the corrected $\mathrm{Rb}$ primers (originally designed from the consensus sequences of vertebrate and invertebrate species; corrected from the returned cDNA sequence) from total RNA prepared from crayfish tail muscle and hepatopancreas tissues. The product was analyzed by BLAST, and did not appear to conform to other $\mathrm{Rb}$ sequences. The non-specific identity of the product is in keeping with low homologies for other crustacean gene products (there are very limited amounts of data about crustacean sequences in gene banks), and as such will be identified as a $\mathrm{Rb}$ like protein. FIG.6.8A shows the partial nucleotide sequence with the corresponding amino acid sequence; the partial crayfish amino acid sequence was 53 amino acids. FIG.6.8B shows the homology between the isolated cDNA from $O$. virilis and the comparable fragment from the water flea. FIG 6.8C shows a homology tree produced from the alignment of the partial $O$. virilis $R b$-like cDNA sequence with $\mathrm{Rb}$ from zebrafish (Danio rerio) and water flea (Daphnia pulex). The homology tree shows the separation of sequence homology, between the crustaceans, there is $47 \%$ similarity, which drops to $36 \%$ similarity between the zebrafish and the invertebrates.

\subsubsection{Transcript expression of $R b$-like protein in response to anoxia}

Changes in the relative transcript levels of $\mathrm{Rb}$-like protein in response to $20 \mathrm{~h}$ of anoxia were assessed using RT-PCR and perfect primers designed from the crayfish sequence 
(FIG.6.9). The representative RT-PCR bands of Rb amplicons (shown below the histogram), show, in conjunction with the above graphical representation, the changes in the relative levels of these transcripts in response to anoxia. $\mathrm{Rb}$ transcript levels were normalized with tubulin bands corresponding to the same tissue samples. $\mathrm{Rb}$ transcript levels showed significant increases in both tail muscle and hepatopancreas tissues, in response to anoxia; $1.6 \pm 0.05$ fold and $1.2 \pm 0.06$ fold respectively (both $\mathrm{P}<0.05$ )

\subsubsection{Phosphorylation of $R b$, and protein expression, cellular localization and DNA} binding activity of E2F transcription factors in response to anoxia

The retinoblastoma protein, through binding of the E2F transcription factors, prevents progression of the cell cycle. Cyclin D/CDK4/6 complexes are able to phosphorylate the serine 780 residue of $\mathrm{Rb}$, preventing its ability to bind the E2Fs, allowing progression through the $G_{1} / S$ barrier. Nuclear localization of the E2F transcription factors allows transactivation of cell cycle targets.

Changes in $\mathrm{Rb}$ phosphorylation of serine 780 (FIG 6.10), in response to anoxia were measured using immunoblotting. Polyclonal Rb-p (S780) antibodies cross reacted with protein bands of $\sim 110 \mathrm{kDa}$, respectively, which is their expected size.

Phosphorylation of $\mathrm{Rb}$ increased significantly in both tail muscle (3.8 \pm 0.3 fold $)$ and hepatopancreas $(2.0 \pm 0.1$ fold) under anoxia (both $P<0.05)$.

Changes of E2F-4 protein expression in nuclear extracts (FIG 6.11) were measured in response to anoxia using immunoblotting. Polyclonal E2F-4 antibodies cross reacted with protein bands of $\sim 45 \mathrm{kDa}$, which was their expected size. Protein levels, in both nuclear extracts of tail muscle and hepatopancreas showed no changes in response to anoxia. 
Changes of E2F-1 protein expression in total and nuclear extracts (FIG 6.12) were measured in response to anoxia using immunoblotting. Polyclonal E2F-1 antibodies cross reacted with protein bands of $\sim 70 \mathrm{kDa}$, which was their expected size. In the muscle, while there were no changes seen in total E2F-1 protein levels, with anoxia, there were significant increases in nuclear E2F-1 protein levels $(3.0 \pm 0.3$ fold; $P<0.05)$. In the hepatopancreas however, total protein level increase $(1.4 \pm 0.1$ fold; $P<0.05)$ with anoxia, were mirrored by nuclear level increases $(1.8 \pm 0.02$ fold; $P<0.05)$ during anoxia.

Changes in the DNA-binding activity of nuclear E2F-4 and E2F-1 in response to anoxia were assessed using a transcription factor binding assay for tail muscle and hepatopancreas tissues (FIG.6.13). For E2F-4, the relative amount of DNA binding in the tail muscle, in conjunction with the nuclear-profiling of the protein, there were no significant changes in DNA binding seen in response to anoxia. Conversely, E2F-1 in the tail muscle, showed a slight but significant increase $(1.1 \pm 0.02$ fold $)$ in DNA binding activity in conjunction with increased nuclear protein presence, with anoxia. Interestingly, despite, increased nuclear protein levels in the hepatopancreas, there was no change in DNA binding activity, with anoxic exposure.

\subsubsection{Phosphorylation and protein expression of cell cycle checkpoint regulators in} response to anoxia

Phospho control of the cell cycle is crucial to its progression. Certain cyclin/CDK complex phosphorylation can lead to inhibition, with dephosphorylation re-activating them. The checkpoint kinases, Chk1 and Chk2, are regulators of cell cycle progression in response to DNA damage. The Chk's are activated by phosphorylation, and are often 
triggered by ATM/ATR kinases as a part of stress response. Chk1 phosphorylated at serine 317 , targets members of the cdc25 family of phosphatases for inactivation via phosphorylation. Likewise, Chk2 phosphorylated at serine 19, is targeted for activation by the canonical ATM/ATR DNA damage response pathway. Cdc25C is a member of the cdc25 phosphatase family which is responsible for maintaining cdc2 (CDK1) activity and subsequent progression into mitosis. Phosphorylation of cdc25C determines progression of cells through the $\mathrm{G}_{2} / \mathrm{M}$ barrier.

Phosphorylation of Chk1 at serine 317 and Chk2 at serine 19, in response to anoxia, was measured using immunoblotting (FIG 6. 14). Polyclonal antibodies for Chk1-p (S317) and Chk2-p (S19) cross reacted with bands of $\sim 55$ and $60 \mathrm{kDa}$, respectively, which were their respective sizes. Chk1 phosphorylation increased significantly, under anoxia, in both tissues; $1.5 \pm 0.05$ fold and $2.3 \pm 0.15$, respectively (both $P<0.05)$. Chk2 phosphorylation increased $(1.4 \pm 0.06$ fold) in the hepatopancreas, as with Chk1, under anoxia. Conversely, in the tail muscle, phosphorylation of Chk2 at serine 19 decreased $25 \pm 3 \%$ with anoxia.

Protein levels of cdc25C were measured in nuclear fractions, with translocation to the nucleus seen prior to mitotic induction (FIG. 6.15). Protein levels, in response to anoxia, were assessed with immunoblotting. Polyclonal cdc25C antibodies cross reacted with protein bands $\sim 65 \mathrm{kDa}$, which were their expected size. Under anoxia, protein levels in the nucleus were not seen to change significantly, in either tissue.

\subsubsection{Phosphorylation, protein expression and cellular localization of cell cycle} inhibitors in response to anoxia 
Cell cycle inhibition is regulated by the cyclin inhibiting proteins (Cip) and kinase inhibiting proteins (Kip); targeting the cyclin/CDK complexes. $\mathrm{P} 15 / 16$ is part of the 'inhibitors of CDK4' (INK4) family of cell cycle inhibitors. P16 specifically inhibits cyclin $\mathrm{D}$, forming an inactive cyclinD-p16-CDK4 complex and preventing progression into the S phase of the cell cycle. Phosphorylation of p16 at serine 152 enhances association with CDK4. P21 is a Cip member of inhibitors, which hold $\mathrm{G}_{1}$ as a restriction point of the cell cycle. Active p21 associates with $\mathrm{CDK} 2$ complexes, preventing their activity. Alternately, $\mathrm{p} 21$ phosphorylated at threonine 145 decreases CDK2 association as well as DNA polymerase subunit (PCNA) binding, which typically inhibits DNA replication. Nuclear localization of $\mathrm{p} 21$ is correlated with increased cell cycle inhibition. P27, a Kip cell cycle inhibitor, inhibits cyclin E/CDK2 complexes.

P15/16 phosphorylation and protein level increases were assessed in response to anoxia, using immunoblotting (FIG. 6.16). Polyclonal antibodies for p15/16 and p16-p (S152) cross reacted with protein bands $\sim 16 \mathrm{kDa}$, which were their expected size. While in the tail muscle, protein levels did not change with anoxia, phosphorylation of serine 152 increased significantly $(1.8 \pm 0.09$ fold; $P<0.05)$. In the hepatopancreas, both protein levels $(1.4 \pm 0.1$ fold; $P<0.05)$ and phosphorylation $(2.2 \pm 0.4$ fold $)$ increased significantly with anoxic exposure.

Phosphorylation of threonine 145 of $\mathrm{p} 21$ was measured in total and nuclear fractions, in response to anoxia, by immunoblotting (FIG. 6. 17). Polyclonal antibodies for $\mathrm{p} 21-\mathrm{p}$ (T145) cross reacted with protein bands of $\sim 21 \mathrm{kDa}$, which were their respected size. In the tail muscle, phosphorylation increased in both total and nuclear fractions (1.5 
\pm 0.05 fold and $1.7 \pm 0.08$ fold respectively; $\mathrm{P}<0.05$ ), under anoxia, while there were no changes phosphorylation levels in the hepatopancreas, in either fraction.

P27 protein expression in response to anoxia was assessed using immunoblotting (FIG. 6.18). Polyclonal antibodies for $\mathrm{p} 27$ cross reacted with protein bands of $\sim 27 \mathrm{kDa}$, which were their expected size. Protein levels were not seen to change in either tail muscle or hepatopancreas tissues, under anoxia.

\subsection{Discussion}

The process of the cell cycle is finely regulated to control rates of proliferation and to ensure that cells are produced without significant mutations. Standard regulation of the cell cycle is, however, susceptible to alteration by various stress conditions in many different organisms, including fungi (Gray et al., 2004), plants (Sano et al., 2006) and animals (Biggar and Storey, 2009). Under the energy limited conditions of a prolonged anoxic exposure, energetically expensive processes are often seen to be minimized, in order to maintain energetic homeostasis. Along with ion channel regulation and protein synthesis, cellular proliferation is particularly costly, and there are already proposed correlations with reduced energy status and cellular quiescence (Mazia, 1962). Despite this, and the hypothesis that echoed these thoughts, the cell cycle of the crayfish did not appear to attenuate under anoxia (Table 6.3).

Oxygen deprivation and the cell cycle

How exactly the cell cycle is regulated in models of metabolic rate depression, animals capable of entering hypometabolic states, is still at the introductory stage (Biggar and Storey, 2009). Indeed, the mechanism of cell cycle regulation in response to oxygen deprivation is still being elucidated, but what is initially clear is the distinct regulatory 
pattern of quiescence. From initial studies, cell cycle arrest is possible particularly at the $\mathrm{G}_{1}$ phase, by halting progression into DNA replication. What's more, a number of animals are seen to do just this in response to hypoxia and anoxia (see Appendix B.7). Despite a range of examples, these studies are typically undertaken in developing (often embryonic) cells. There are, alternatively, virtually no studies of cell cycle regulation in the mature cells of stress adapted (particularly anoxia resistant) animals. Hence cell cycle studies during anoxia, in the fresh water crayfish $O$. virilis could be both novel and instructive for future cell cycle studies in models of hypometabolism. The reach of such studies also extends to the medical arena, in which notably the cell cycle regulation of hypoxic tumor cells is already a rich area of research (Olcina et al., 2010; Rodrigues et al, 2008; DeBerardinis et al., 2008). Tumor cells are highly sensitive to oxygen deprivation and as cell proliferation occurs faster than vasculature can develop, cells at the edge of tumor formation can become hypoxic; under which condition, replication of the tumor cells is arrested. Hence during the hypoxic state, and more pertinently during reoxygenation, the ability to re-enter a dividing state is highly important. Hypoxia/Reoxygenation induced DNA damage would canonically induce ATM/ATR activity, subsequently acting on Chk1 and 2, so the capacity of tumor cells to mediate this DNA damage response is crucial to their survival and proliferation (Olcina et al., 2010). Survival of the hypoxic state, in tumor cells, is mediated by many molecular changes, with some of the targets being the primary mediators of cellular survival and proliferation, such as Akt and mTOR (DeBerardinis et al., 2008). Inactivating (ex. by loss of gene function) their inhibitors, such as the PTEN phosphatase, not only has effects on carbohydrate metabolism (Buzzai et al., 2005), but on their specific cell cycle targets 
such as the cell cycle inhibitors. Similarly, activity of the myc family of genes (including $\mathrm{c}-\mathrm{Myc}$ ) is often found to be amplified in tumors. As mentioned briefly above, c-Myc is a cell cycle promoter and increases cyclin/CDK activity to influence progression through the $G_{1} / S$ phase barrier. C-Myc is also critically involved in transactivating a number of $S$ phase targets involved in nucleotide and one-carbon metabolism which are necessary for transition into the $\mathrm{G}_{2}$ phase (DeBerardinis et al., 2008). Hence, while extremely complex, regulation of cellular proliferation in tumor cells shows significant overlap with regulatory elements in both stress adapted and non-stress adapted models.

Regulation of the $G_{1} / S$ barrier in $O$. virilis, under anoxia

The $\mathrm{G}_{1} / \mathrm{S}$ transition of the cell cycle involves, primarily, the activation of cyclin $\mathrm{E} / \mathrm{CDK} 2$ and cyclin $\mathrm{D} / \mathrm{CDK} 4 / 6$ complexes in order to phosphorylate $\mathrm{Rb}$, and allow active $\mathrm{E} 2 \mathrm{~F}$ ('activating' class) transcription factors to transactivate S-phase targets. These cyclin/CDK complexes can be inhibited by members of the INK4, Cip and Kip cell cycle inhibitors, along with the checkpoint kinases, through cdc25A repression.

The cyclin/CDK complexes, being the major players in cell cycle progression, are targets of repression by numerous factors. At the $\mathrm{G}_{1} / \mathrm{S}$ phase, both cyclin $\mathrm{D} / \mathrm{CDK} 4 / 6$ and cyclin $\mathrm{E} / \mathrm{CDK} 2$ phosphorylation of $\mathrm{Rb}$ is thought to be necessary for its complete inhibition (Lundberg and Weinberg, 1998). By preventing association with one or the other component, correct phosphorylation state, or correct subcellular location, cyclin/CDK complex activity can be minimized. Cellular location, in either cytoplasmic or nuclear fractions, may confer different activities of the cyclin/CDK complexes, which are able to shuttle between the two. Indeed cyclins are predominantly nuclear, and those particularly involved in DNA replication (cyclin A and E) have links to centrosome 
replication and are seen to accumulate (in complex) in the nucleus as part of a functional cell cycle (Jackman et al., 2002). In the tail muscle of $O$. virilis there were significant increases in protein levels of CDK2, 4 and 6, while in the hepatopancreas CDK4 and 6 increased in response to anoxia. In the case of CDK6, there were anoxia triggered increases in the nucleus of the tail muscle, as well as in both the cytoplasmic and nuclear subcellular fractions of the hepatopancreas. Indeed it is thought that only the nuclear form of the cyclin D/CDK6 complex is active, and capable of Rb phosphorylation (Mahony et al., 1998).

Cyclin $E$, in association with $C D K 2$ is $\mathrm{G}_{\mathrm{l}} / \mathrm{S}$ transition specific, and shows maximal accumulation during the $\mathrm{G}_{1}$ phase (Koff et al., 1992). O. virilis cyclin $\mathrm{E}$ did not appear to change significantly in either tissue or fraction within the cell. This static profile, while not indicative of a resultant shortened $G_{1}$ phase, as is implicated with increased cyclin expression, may simply be suggestive of unaltered progression rates through this barrier. It is also not necessary for cyclin $\mathrm{E}$ to complex with CDK2 for nuclear localization (Jackman et al., 2002), indicating that the lack of change in nuclear localization would not affect the activity profile of its complex. However, cyclin D was seen to change significantly in response to anoxia. In the hepatopancreas, following increases in both CDK4 and 6, there were increased protein levels of both cytoplasmic and nuclear cyclin. In the muscle, however, while cytoplasmic cyclin D levels did not change, nuclear cyclin D was shown to decrease.

Despite the decrease in CDK2 expression in the hepatopancreas, and reduced nuclear cyclin $D$ in the tail muscle, there appears to be a fairly strong profile of $G_{1} / S$ specific cyclin/CDK complex activity under anoxia. The phosphorylation of $\mathrm{Rb}$ seems to 
confirm this with significant increases in both tissues. Phosphorylation at serine 780 , is a site particularly targeted by cyclin D/CDK4/6, which aligns with the CDK expression. What is interesting is that $\mathrm{Rb}$ transcript levels are seen to increase during anoxia in both tissues, potentially indicating that phosphorylation increases are simply linked to increased protein levels, and not a separate event.

Following retinoblastoma expression and regulation, in the static expression of 'repressive' E2F (4) and increased nuclear 'activating' E2F (1) in both tissues, an overall profile of $\mathrm{Rb}$ repression is formed. DNA binding activity of nuclear E2Fs is complementary for E2F-4 (no change), but in the tail muscle, E2F-1 binding was shown to increase (in conjunction with increased nuclear protein levels). In the hepatopancreas, despite E2F-1 nuclear protein level increases, there was no change in DNA binding activity from the control level.

In terms of the inhibitors that affect $\mathrm{G}_{1} / \mathrm{S}$ expression, the profile appeared mixed. Inhibition of the p27 Kip, which targets CDK 2 and 4 in beta-cells during embryogenesis and maintains cellular quiescence, is seen to confer re-entry into the cell cycle (Georgia and Bhushan, 2006). In O. virilis under anoxia, there were no changes seen in $\mathrm{p} 27$ protein expression. The p21 Cip, which localizes to the nucleus in response to checkpoint mediated signaling to arrest the cell cycle at $\mathrm{G}_{1}$, can be regulated by phosphorylation at threonine 145 by Akt. This prevents not only its inhibitory binding to cyclin $E / C D K 2$, but its binding to the proliferating cell nuclear antigen (PCNA), which inhibits DNA replication (Rössig et al., 2001). In the $O$. virilis tail muscle, both total soluble fraction and nuclear subfraction indicate increased $\mathrm{p} 21$ phosphorylation, while in the hepatopancreas, there is no such change. Also in control of the $G_{1} / S$ transition are the 
INK4 members p15 and 16. These kinase inhibitors are capable of binding CDK4/6 to regulate cell cycle progression, and can have specific binding activity through phosphorylation of the serine 152 residue. The phosphorylated form of p16 preferentially associates with $\mathrm{CDK} 4$, although it is not determined if phosphorylation occurs following kinase binding (Gump et al., 2003). Under anoxia, in the crayfish, protein expression of p15/16 did not change in the tail muscle, but increased significantly in the hepatopancreas. Phosphorylation of serine 152, was however seen to increase in both tissues. While in the hepatopancreas, this phosphorylation event seems to correlate with total protein increases, in the tail muscle, there may be a significant targeting of CDK4 under anoxia.

In general, both tissues exhibit $\mathrm{G}_{1} / \mathrm{S}$ progression through the standard pattern of cyclin/CDK activity, Rb phosphorylation and enhanced presence of nuclear E2F-1 (Table 6.3). In the tail muscle, there are even signs of enhanced $G_{1} / S$ progression under anoxia; increased E2F-1 DNA binding and increased p21 phosphorylation. In the hepatopancreas, however, the cell cycle does not necessarily appear to be enhanced. Particularly, the decrease in CDK2 protein levels, along with unenhanced E2F-1 DNA binding, and no changes in $\mathrm{p} 21$ inhibition. Despite this, level expression of p27 and enhanced p16 profiling, indicates that there is still significant regulation of the $G_{1} / S$ barrier.

Regulation of $G_{2}$ and mitosis in $O$. virilis, under anoxia

The $\mathrm{G}_{2} / \mathrm{M}$ phase barrier into mitosis is mediated by both cyclin A/CDK2 and cyclin $\mathrm{B} / \mathrm{CDK} 1$ complexes. While mitosis is dependent on cyclin $\mathrm{B} / \mathrm{CDK} 1$, movement into $\mathrm{G} 2$ requires cyclin $\mathrm{A} / \mathrm{CDK} 2$ activity to move through the S-phase. Nuclear 
localization of cyclin A is linked to its ability to bind (and activate) CDK2, possibly suggestive of the need to form complexes prior to nuclear localization (Maridor et al., 1993). Under anoxia, in the crayfish, cyclin A levels showed parallel activity in both tissues, with significant increases in the cytoplasmic fraction, but a static expression profile in nuclear fractions. The increased cyclin A expression in the cytoplasm only, may be indicative of decreased nuclear shuttling. While this correlated with decreased CDK2 expression in the hepatopancreas, the change is unexplained in the tail muscle. Regardless, while there is not enhanced nuclear cyclin A, there still remains a control level, indicating potentially consistent cyclin A complex activity.

Cyclin B, which complexes with CDK1 (aka cdc2), is crucial for mitosis. It typically exhibits high cytoplasmic localization due to extensive nuclear export, but despite this, functional $\mathrm{G}_{2} / \mathrm{M}$ progression is achieved through its import mediated by numerous contributors (Porter and Donoghue, 2005). In the crayfish, cyclin B expression was almost uniformly increased, across tissues and fractions. Apart from in the cytoplasmic fraction of the tail muscle, there was a significant protein level increase in both tissues, in both fractions (cytoplasmic and nuclear).

Mitosis is also controlled by the phospho-state of the cyclin B/CDK1 complex. Activity of the cdc25C phosphatase is a marker of mitotic progression, through its maintenance of the correct phosphorylation state of the cyclin/CDK complex. The Wee kinases are capable of phosphorylating and inactivating cyclin B/CDK1 at threonine 14 and tyrosine 15 , which cdc25C dephosphorylates to maintain complex activity. CyclinB/CDK1 translocates to the nucleus at the end of G2, and similarly, cdc25C is capable of nuclear translocation in order to act on the complex and contribute to mitotic 
initiation. Phosphorylation of cdc25C can either activate or inhibit the phosphatase, depending on the residue targeted. While polo-like kinase 3 (Plk3) phosphorylation of serine 191/198 promotes its nuclear translocation (Bahassi et al., 2004), phosphorylation on serine 216 by the checkpoint kinases (Chk1 and 2) anchors the phosphatase in the cytoplasm to prevent mitosis (Kumagai and Dunphy, 1999; Furnari et al., 1999). Under anoxia in $O$. virilis, in conjunction with increased activity of their canonical activators (ATM and ATR kinases -data shown chapter 4), checkpoint kinase activation (via phosphorylation) was largely seen to increase. Both Chk1 and 2 phosphorylation levels were seen to increase significantly in both tissues, with exception of Chk2 in the tail muscle. Downstream of this, cdc25C nuclear expression was shown to be static. Hence while the checkpoint pathway appears to be active, it does not appear to reduce cdc25C activity.

\section{Conclusions}

While cell cycle regulation in response to anoxia has begun to be elucidated, it is largely only understood in embryonic cells. Regulation to hypoxia, and the ensuing hypometabolism, has been characterized in the nematode $C$. elegans (see Appendix A and B.6), but like embryonic studies in zebrafish and drosophila, the cells in study are not mature. Hence this study of the cell cycle in adult anoxia resistant freshwater crayfish, $O$. virilis, is a new direction in cell cycle regulation. In both of the major regulatory steps, the $G_{1} / S$ and $G_{2} / M$ barriers, the crayfish appears to show progression despite the energetic limitations imposed by oxygen limitation. Major regulatory mechanisms at $\mathrm{G}_{\mathrm{l}}$, such as cyclin D/CDK4/6 accumulation, along with Rb phosphorylation and E2F nuclear localization suggest progression. However, within the hepatopancreas, a slightly 
increased expression/activity profile for the cell cycle inhibitors along with CDK2 reduction, hints at mechanisms keeping the rates of the cell cycle in check. Similarly, the $\mathrm{G}_{2}$ and $\mathrm{M}$ phase markers show progression, with potential rate limiting mechanisms. Cyclin A accumulation in the cytoplasm, as opposed to the nucleus (where it is active), along with reduced CDK2 expression in the hepatopancreas, and Checkpoint kinase activity in both tissues may balance out increased nuclear cyclin B protein levels, to limit the rate and energetic expenditure of mitosis. While the general trend established in the crayfish, under anoxia, was unexpected in terms of the initial hypothesis (and comparison to the dauer model), in conjunction with the pro-developmental signals seen in earlier chapters, it furthers the concept of an active developing cell, even when faced with oxygen deprivation. 


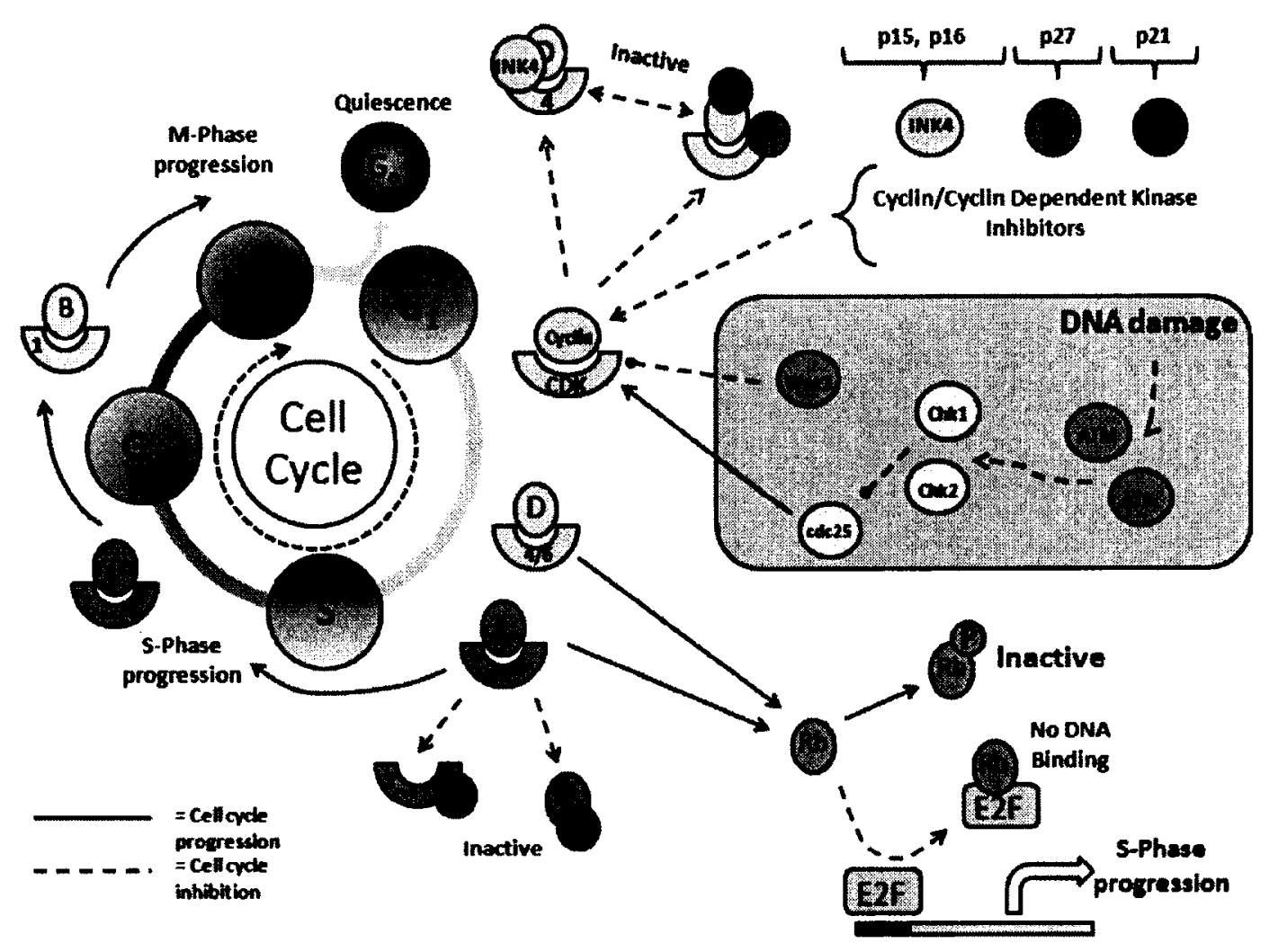

Figure 6.1: An overview of the cell cycle. The cell cycle is primarily controlled by the activities of cyclins and cyclin dependent kinases (CDKs), in complex with each other. Regulatory barriers in transition to DNA replication (S-phase) and mitosis (M-phase) are dependent on cyclin $C D K$ activity to ensure progression of the cycle. At the $G_{1} / S$ barrier control of $\mathrm{Rb}$, which in turn regulate $\mathrm{E} 2 \mathrm{~F}$ transcription factors, is the primary target. Active cyclin/CDK complexes inhibit $\mathrm{Rb}$, allowing E2F activity and $\mathrm{S}$-phase progression. Regulation of the cyclin/CDK complexes comes through cell cycle inhibitors that can bind the components and inactivate the complexes. Cell cycle phospho control is also critical to progression. Checkpoint kinases triggered by DNA damage attenuate the cell cycle, inactivate cdc25 phosphatases, which regulate the phospho-state of cyclin/CDK complexes. 


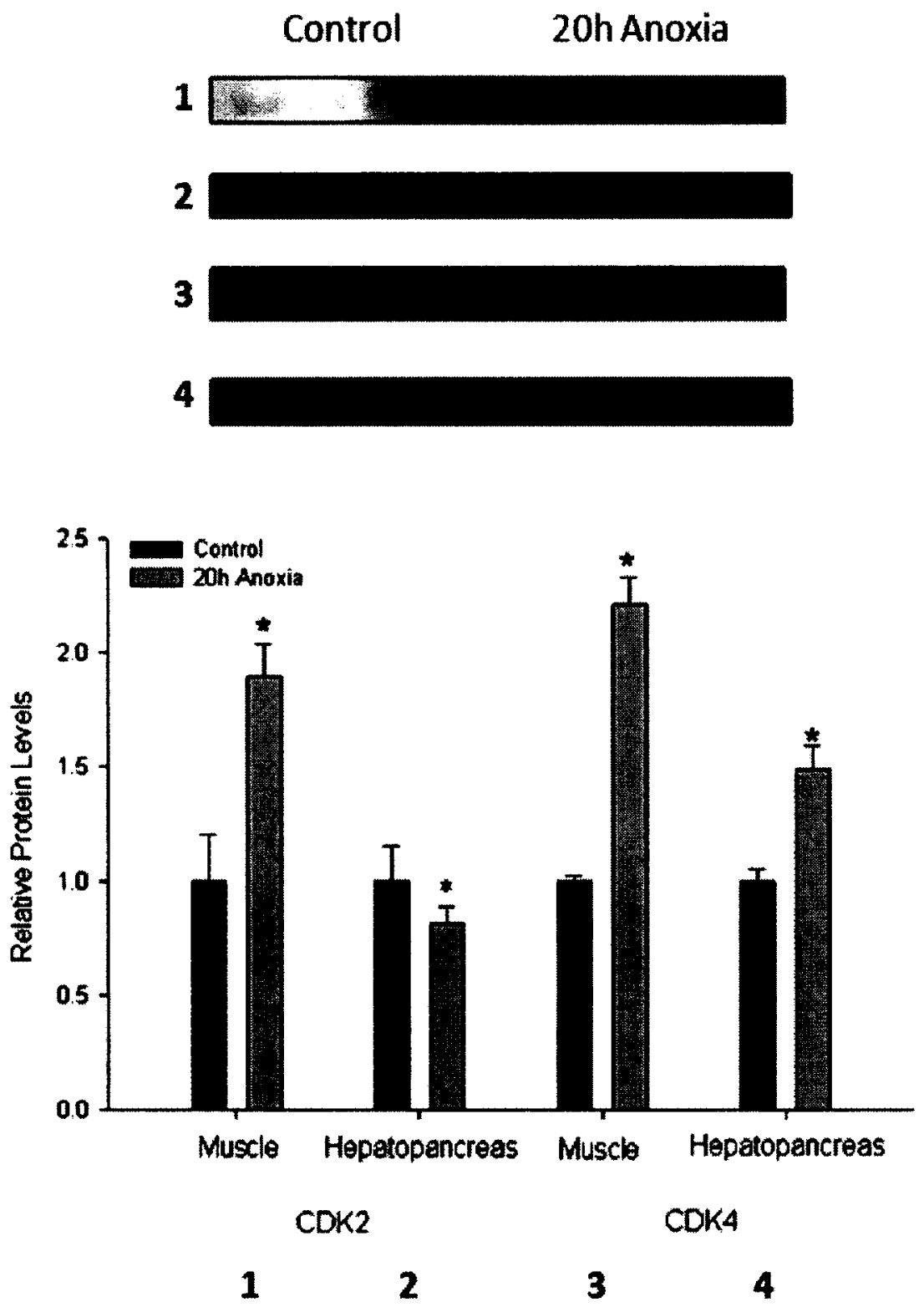

Figure 6.2: Effect of $20 \mathrm{~h}$ anoxia exposure on protein expression of CDK2 and 4 in $O$. virilis tissues.

Protein expression of CDK2 and 4 in total soluble extracts of crayfish tissues in response to anoxia, as determined by western immunoblotting.

Data are means $\pm S E M, n=4$ independent trials for muscle and hepatopancreas. * indicates significantly different from the corresponding control, $P<0.05$. 


\section{Control 20h Anoxia}

1

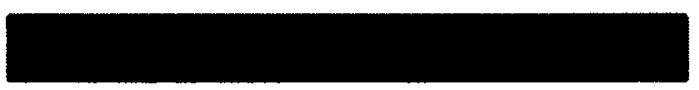

2

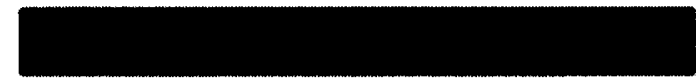

3

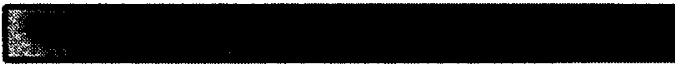

4
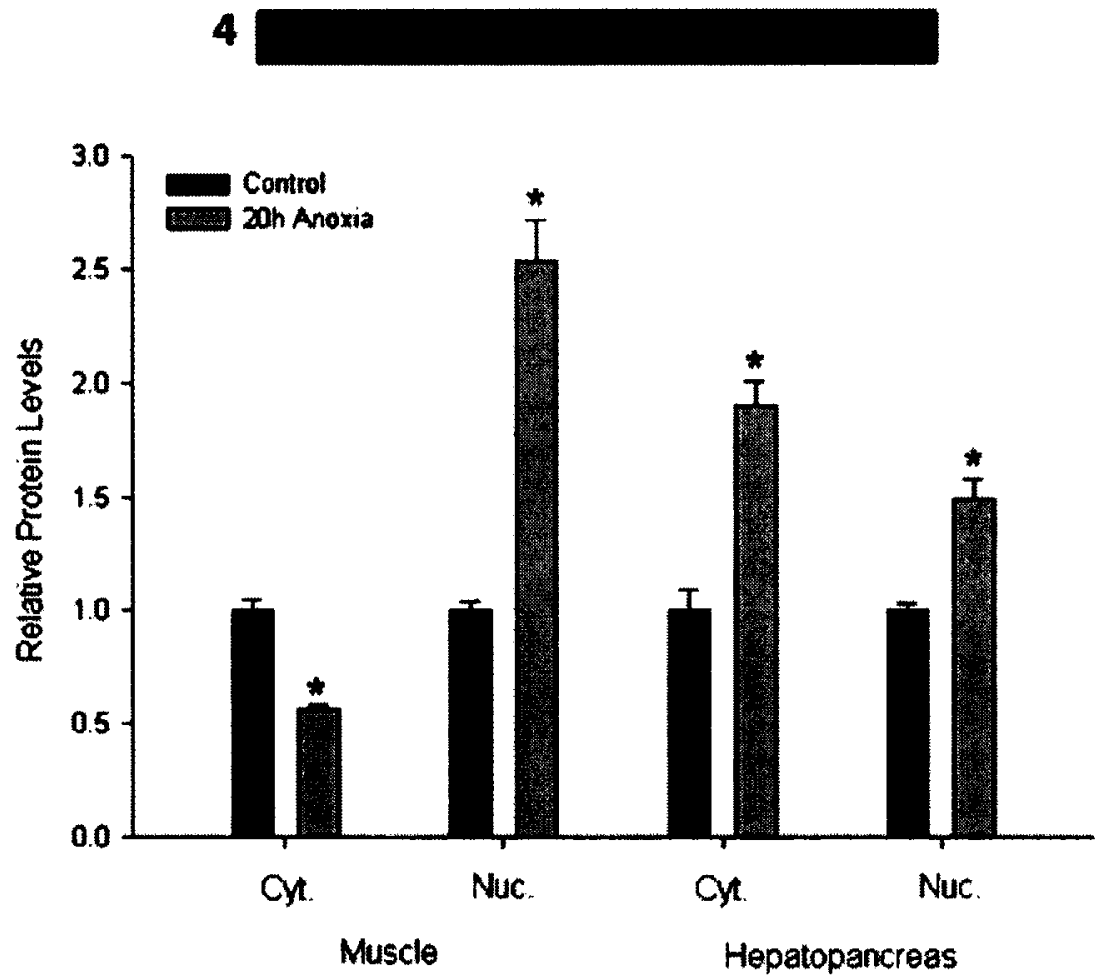

CDK6

$\begin{array}{llll}1 & 2 & 3 & 4\end{array}$

Figure 6.3: Effect of $20 \mathrm{~h}$ anoxia exposure on the protein expression and subcellular distribution of CDK6 in $O$. virilis tissues.

Protein expression of CDK6 in total soluble extracts and nuclear fractions of crayfish tissues (tail muscle, left; hepatopancreas, right) in response to anoxia, as determined by western immunoblotting.

Data are means \pm SEM, $n=4$ independent trials for muscle and hepatopancreas. * indicates significantly different from the corresponding control, $P<0.05$ 


\section{Control 20h Anoxia}

1

2

3

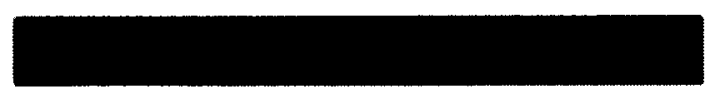

4
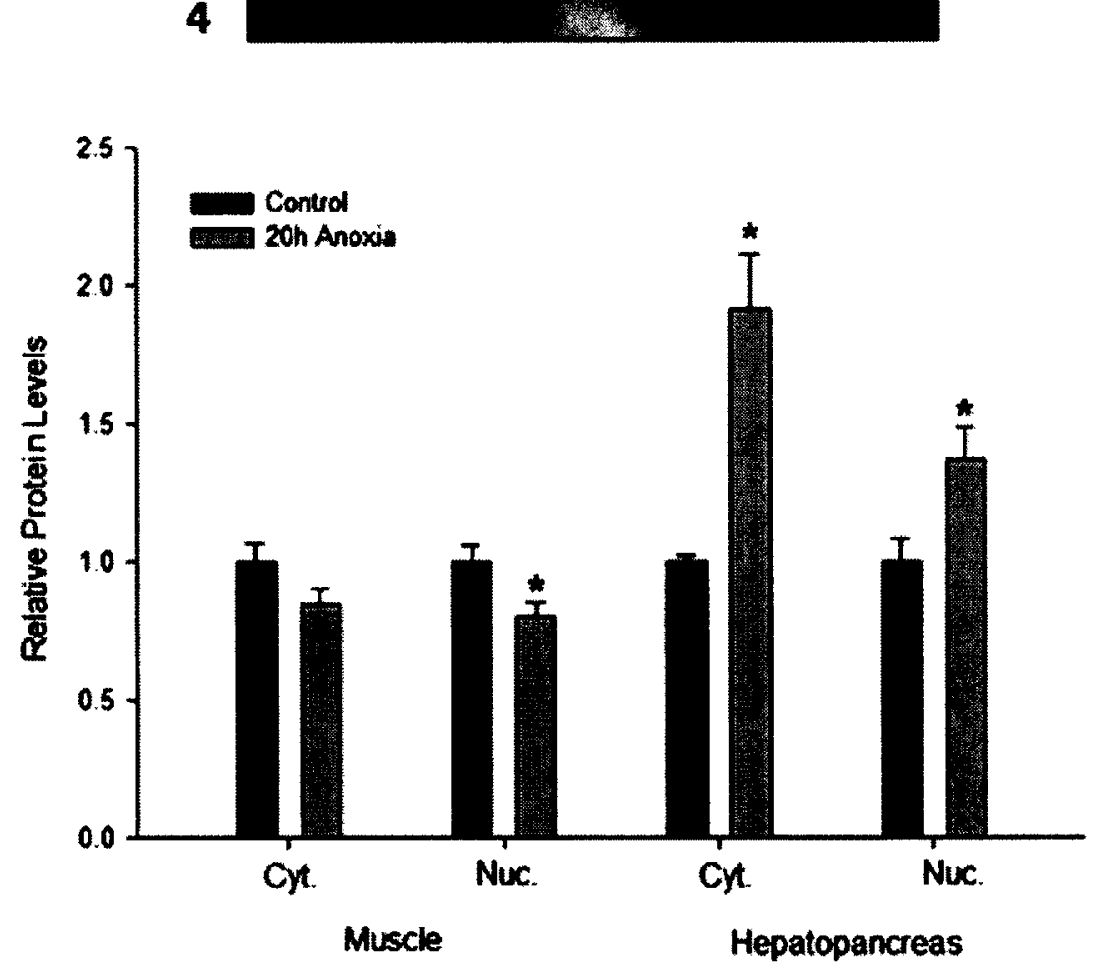

Cyclin D

$\begin{array}{llll}1 & 2 & 3 & 4\end{array}$

Figure 6.4: Effect of $20 \mathrm{~h}$ anoxia exposure on the subcellular distribution of Cyclin $D$ in $O$. virilis tissues.

Protein expression of cyclin D in cytoplasmic and nuclear fractions of crayfish tissues (tail muscle, left; hepatopancreas, right) in response to anoxia, as determined by western immunoblotting.

Data are means \pm SEM, $n=4$ independent trials for muscle and hepatopancreas. * indicates significantly different from the corresponding control, $P<0.05$ 


\section{Control 20h Anoxia}

1 Fre?

2

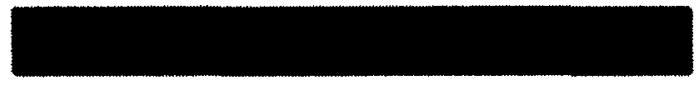

3

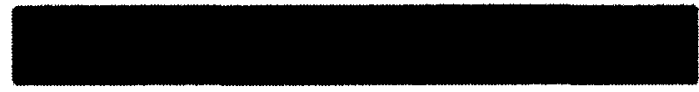

4
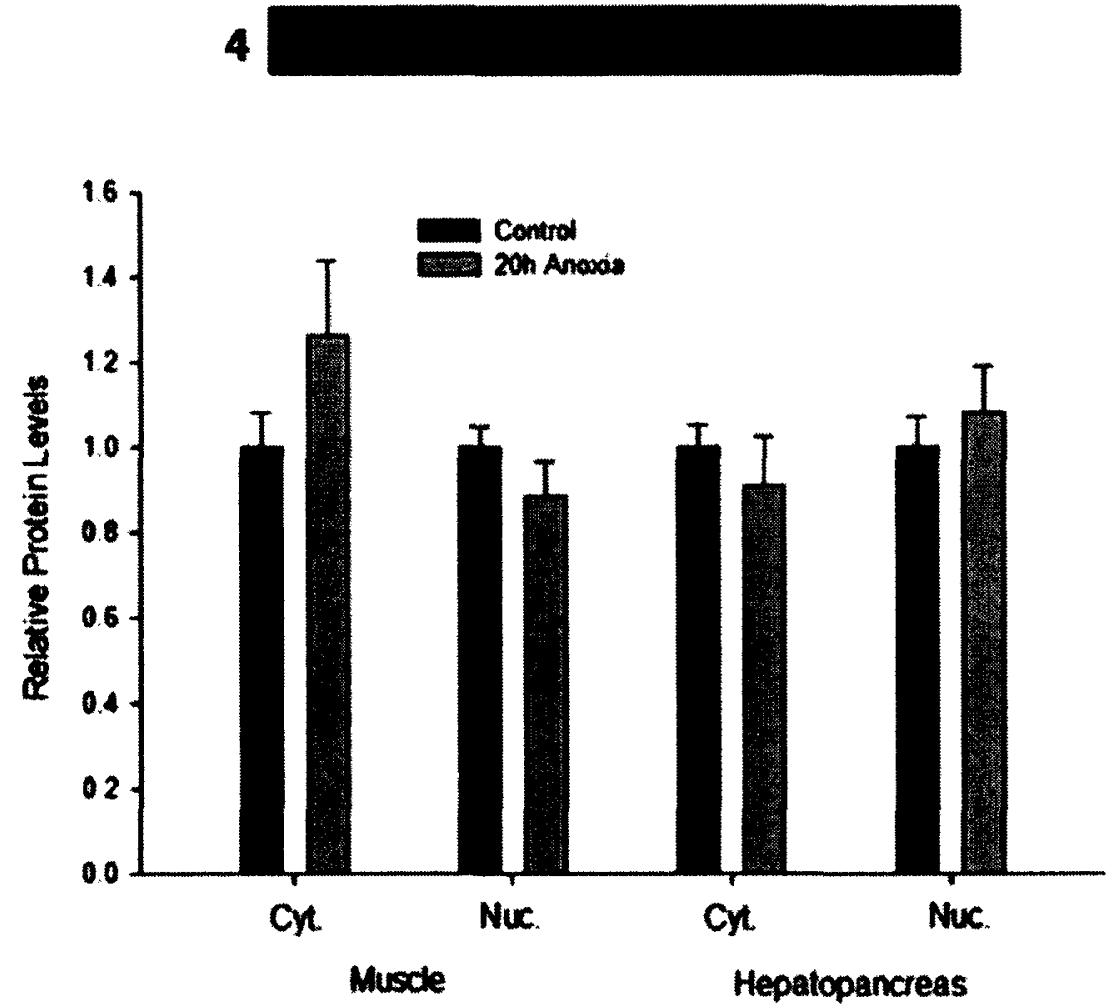

Cycin E

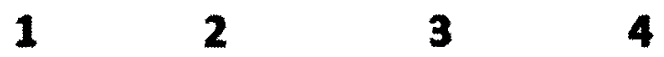

Figure 6.5: Effect of $20 \mathrm{~h}$ anoxia exposure on the subcellular distribution of Cyclin $\mathrm{E}$ in 0 . virilis tissues.

Protein expression of cyclin E in cytoplasmic and nuclear fractions of crayfish tissues (tail muscle, left; hepatopancreas, right) in response to anoxia, as determined by western immunoblotting.

Data are means $\pm S E M, n=4$ independent trials for muscle and hepatopancreas. * indicates significantly different from the corresponding control, $P<0.05$ 


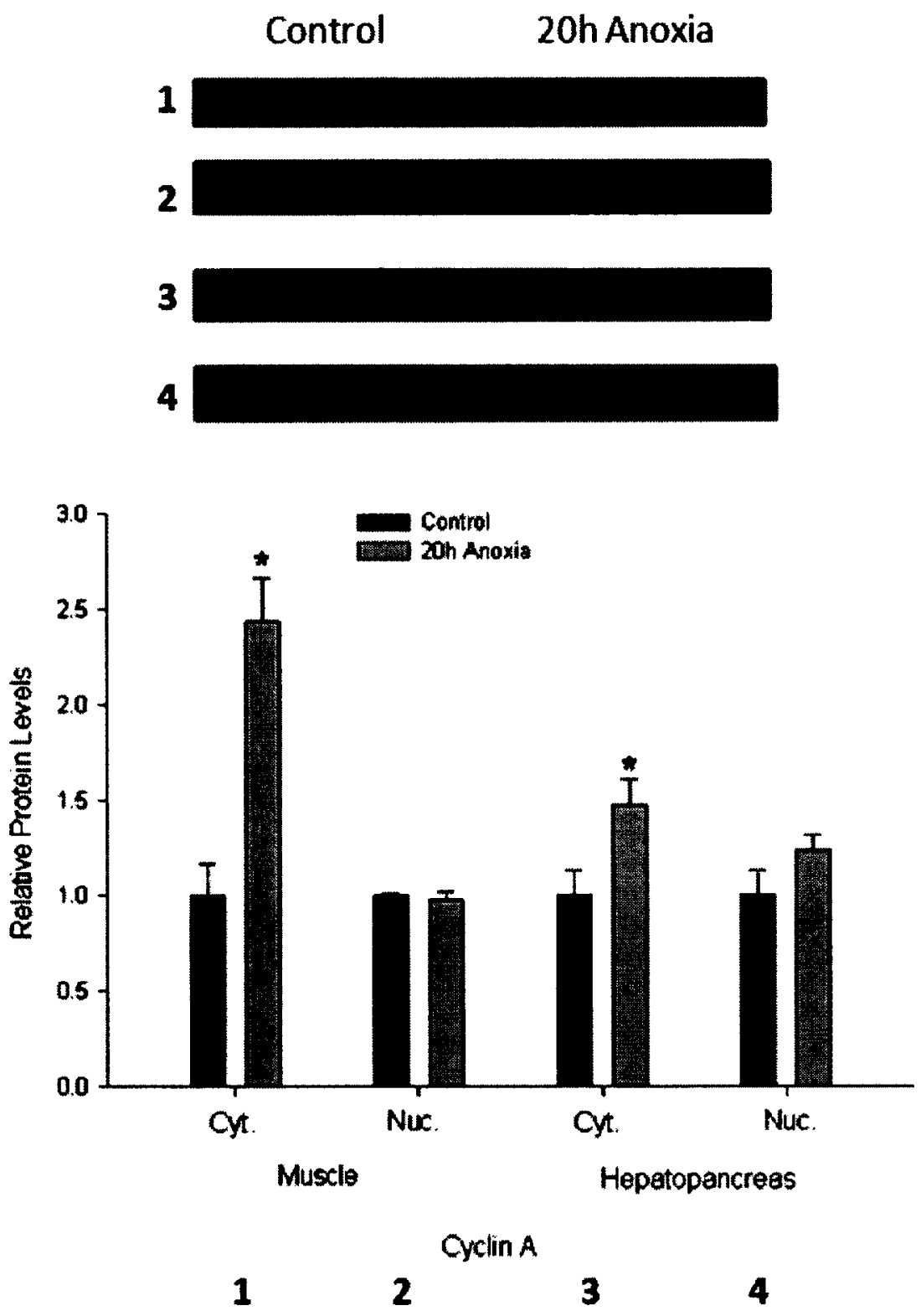

Figure 6.6: Effect of $20 \mathrm{~h}$ anoxia exposure on the subcellular distribution of Cyclin $A$ in $O$. virilis tissues.

Protein expression of cyclin A in cytoplasmic and nuclear fractions of crayfish tissues (tail muscle, left; hepatopancreas, right) in response to anoxia, as determined by western immunoblotting.

Data are means \pm SEM, $n=4$ independent trials for muscle and hepatopancreas. * indicates significantly different from the corresponding control, $P<0.05$ 


\section{Control 20h Anoxia}

\section{1}

2

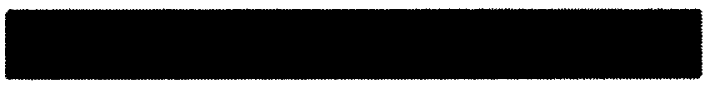

3

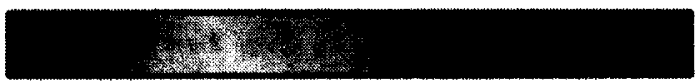

4
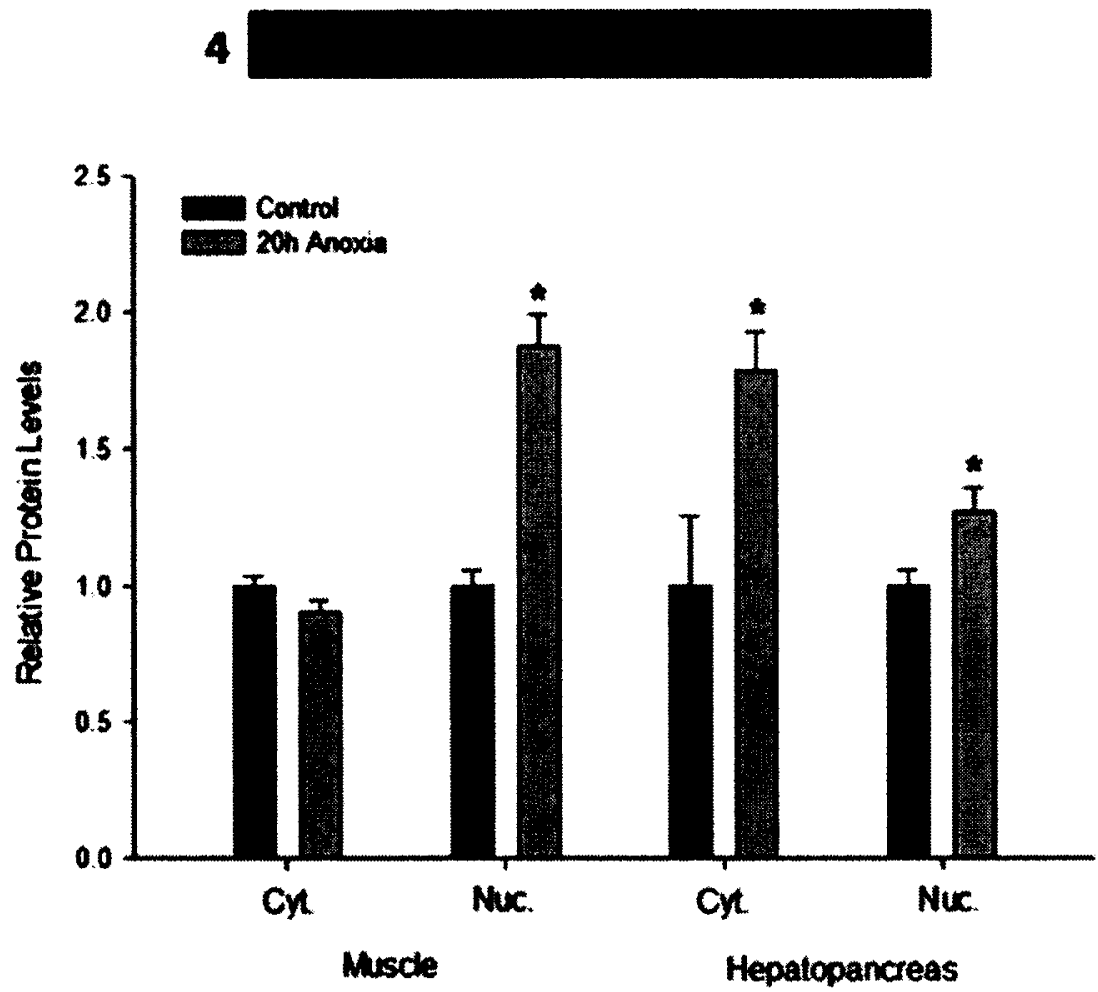

Cyctin B

1

2

3

4

Figure 6.7: Effect of $20 \mathrm{~h}$ anoxia exposure on the subcellular distribution of Cyclin B in 0 . virilis tissues.

Protein expression of cyclin B in cytoplasmic and nuclear fractions of crayfish tissues (tail muscle, left; hepatopancreas, right) in response to anoxia, as determined by western immunoblotting.

Data are means $\pm S E M, n=4$ independent trials for muscle and hepatopancreas. * indicates significantly different from the corresponding control, $P<0.05$ 


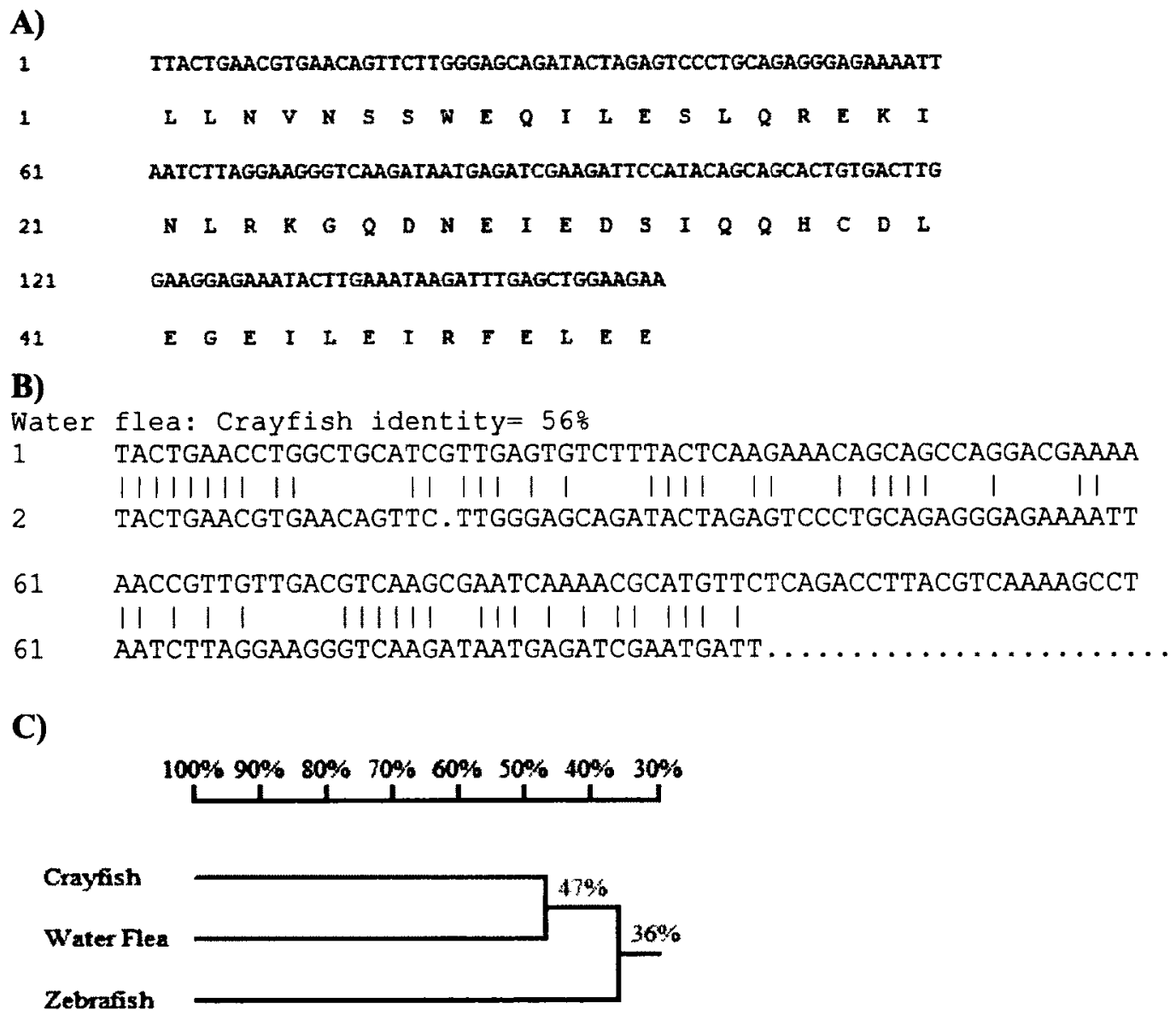

Figure 6.8: Partial nucleotide and deduced amino acid sequence of $O$. virilis Rb-like protein.

(A) The partial cDNA sequence of a $\mathrm{Rb}$ like protein, with the corresponding amino acid sequence is shown. Nucleotides and amino acids are numbered on the left. The nucleotide sequence was 159 nucleotides in length, and encoded 53 amino acids.

(B) Sequence alignment of the isolated crayfish sequence and the corresponding fragment of the water flea sequence.

(C) Homology tree produced from an alignment of the partial $O$. virilis $R b$-like cDNA sequence with $\mathrm{Rb}$ from the water flea (Daphnia pulex) and zebrafish (Danio rerio). The accession for the zebrafish is NM_001077780. There are currently no accession numbers for the sequenced $D$. pulex genes, but sequences may be found at http://wfleabase.org/. The percentage values correspond to the shared identity among the corresponding species. 

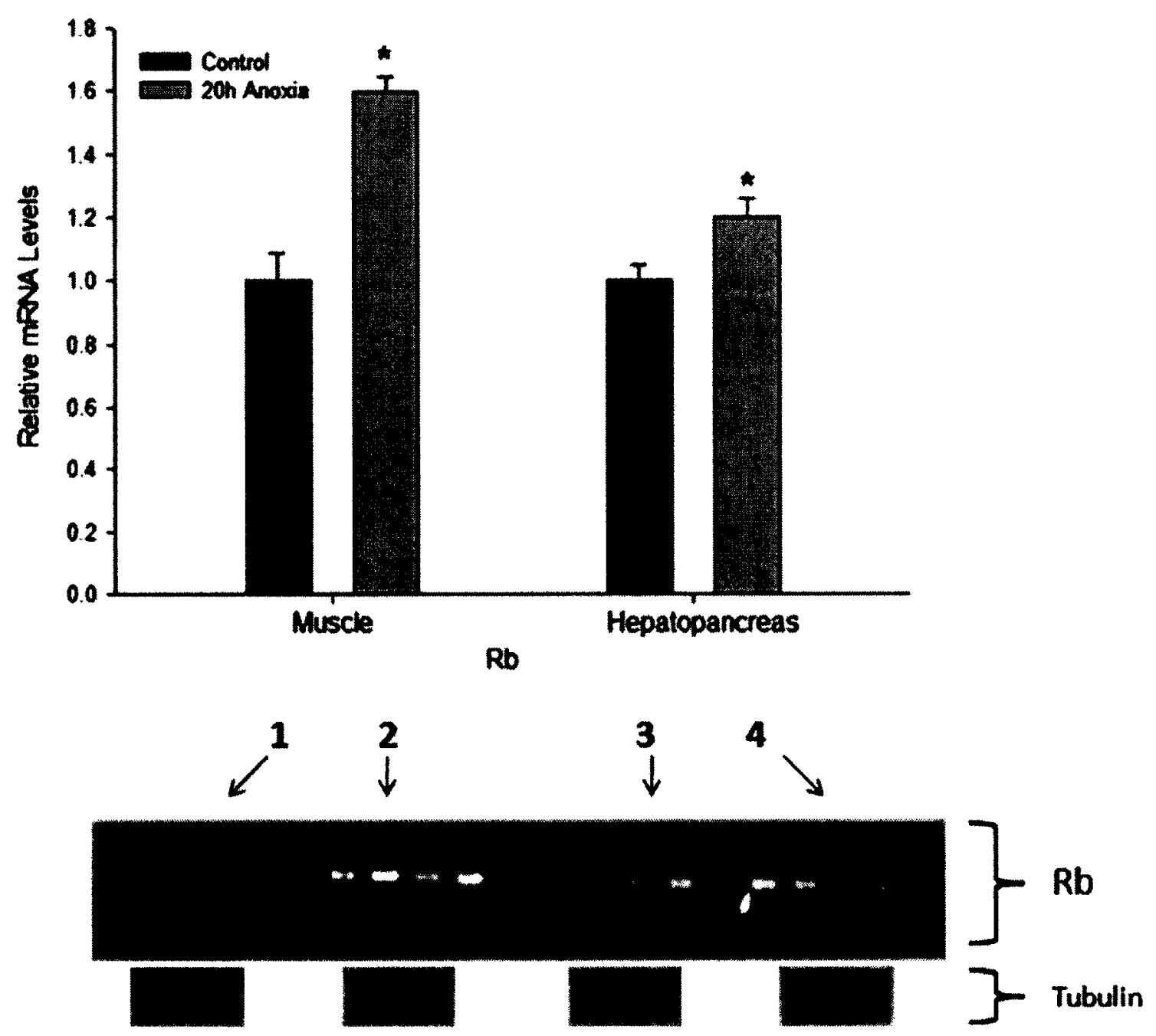

Figure 6.9: Effect of $20 \mathrm{~h}$ anoxic exposure on $R b$-like transcript expression in $O$. virilis tissues as determined by RT-PCR.

Histogram shows the normalized transcript levels under control and anoxic conditions; bands were normalized against the corresponding $\alpha$-tubulin bands (representative from each sample shown) amplified from the same sample. Data are means \pm SEM, $n=4$ independent trials for mRNA isolated from different crayfish. * Indicates significantly different from the corresponding control, $P<0.05$.

$1=$ control muscle, $2=20 \mathrm{~h}$ anoxic muscle, $3=$ control hepatopancreas, $4=20 \mathrm{~h}$ anoxic hepatopancreas

Representative $R b$ amplicons shown below 


\section{Control 20h Anoxia}

1

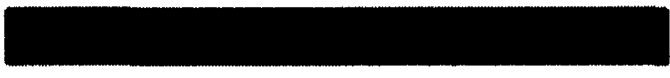

2
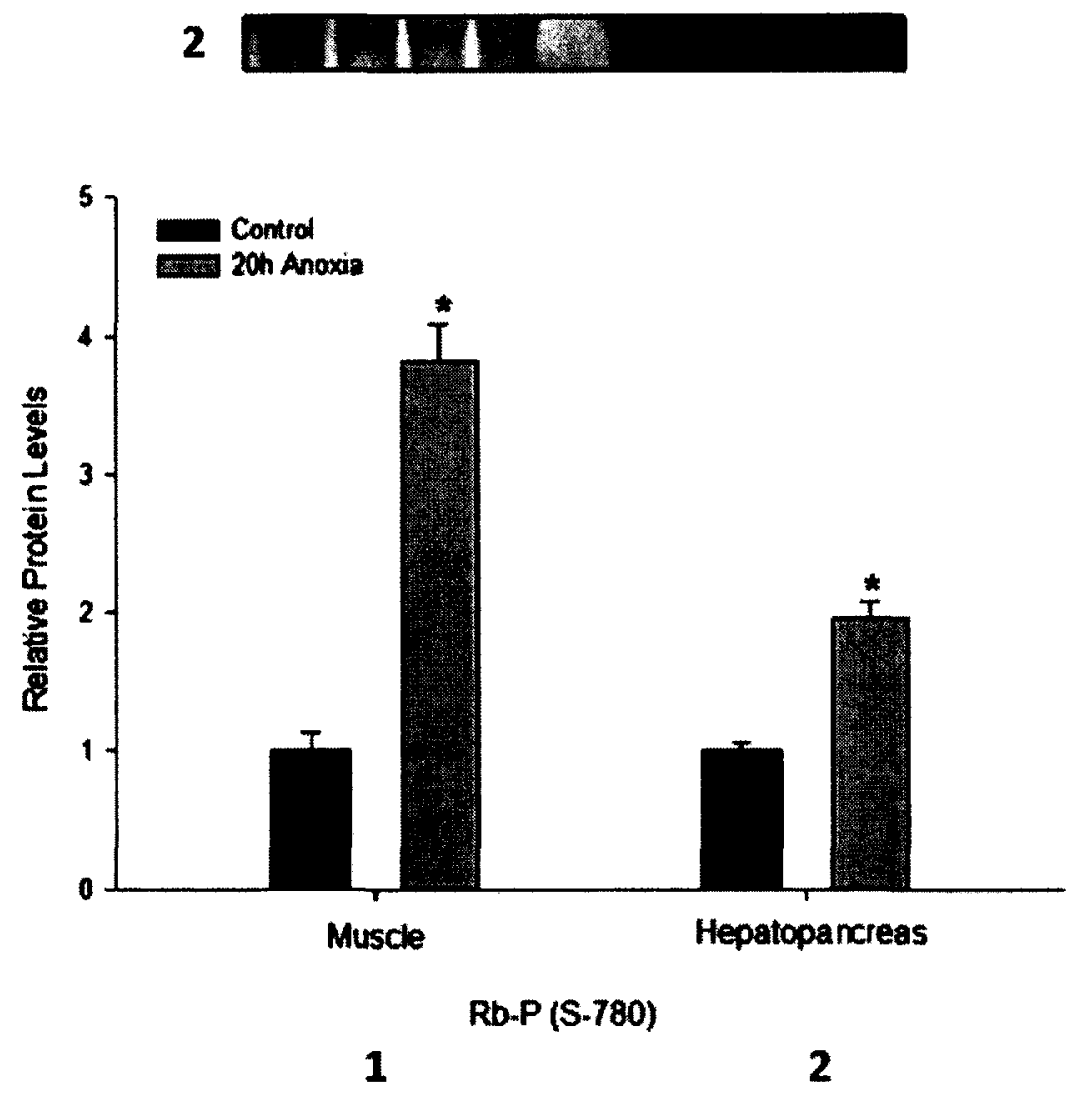

Figure 6.10: Effect of $20 \mathrm{~h}$ anoxia exposure on phosphorylation of Rb (S780) in $O$. virilis tissues.

Changes in relative phosphorylation of $\mathrm{Rb}$ at serine 780 in total soluble extracts of crayfish tissues in response to anoxia, as determined by western immunoblotting. Data are means \pm SEM, $n=4$ independent trials for muscle and hepatopancreas. * indicates significantly different from the corresponding control, $P<0.05$. 


\section{Control 20h Anoxia}

1

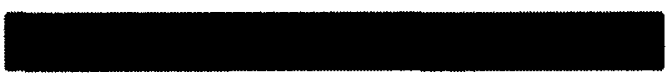

2
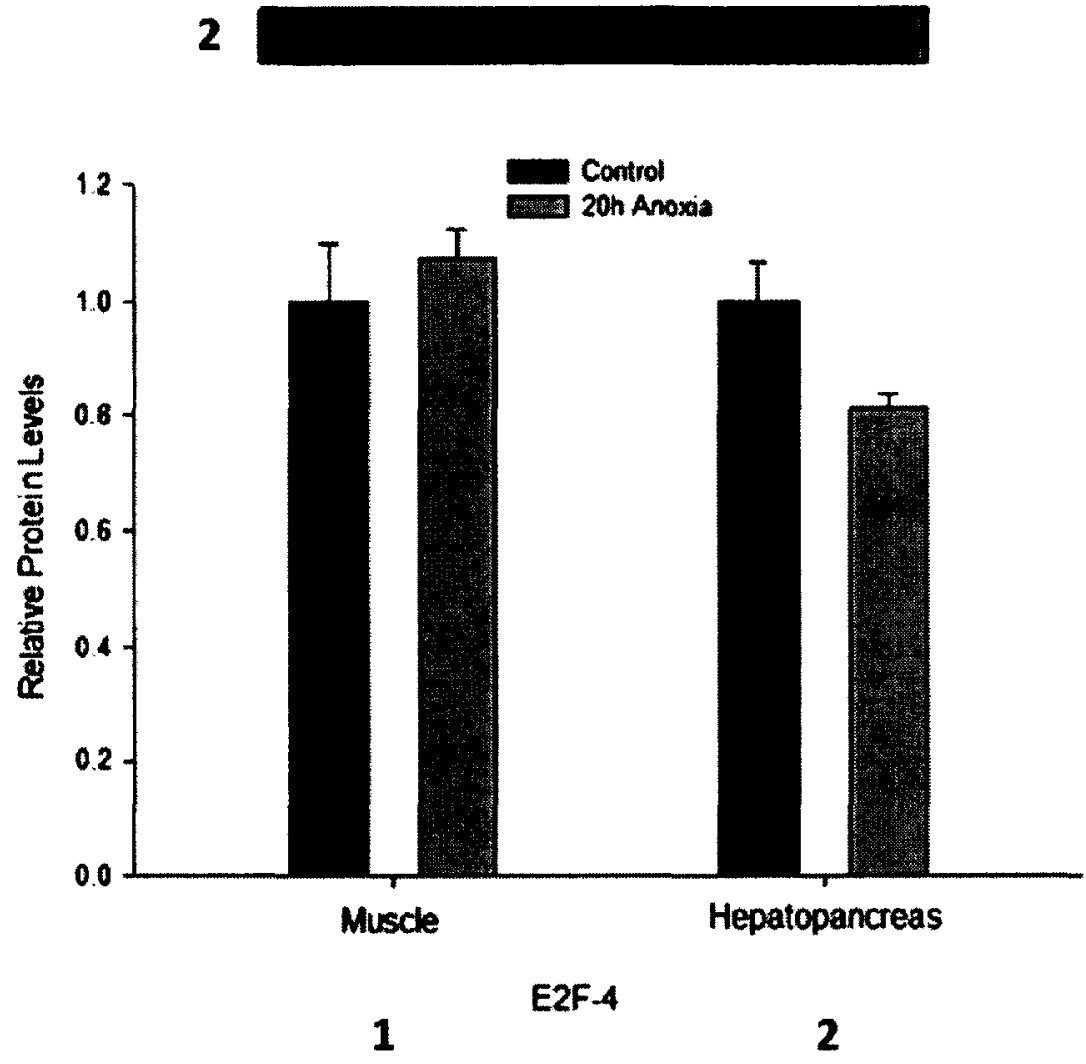

Figure 6.11: Effect of $20 \mathrm{~h}$ anoxia exposure on protein expression of E2F-4 in $\boldsymbol{O}$. virilis tissues.

Changes in relative protein expression of E2F-4 in total soluble extracts of crayfish tissues in response to anoxia, as determined by western immunoblotting. Data are means $\pm S E M, n=4$ independent trials for muscle and hepatopancreas. * indicates significantly different from the corresponding control, $P<0.05$. 


\section{Control 20h Anoxia}

\section{1}

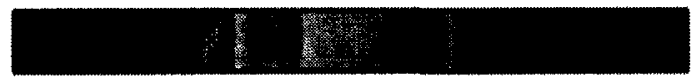

2

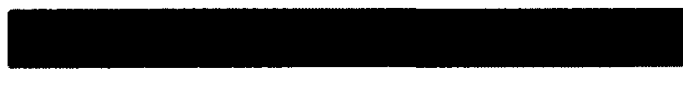

3

4
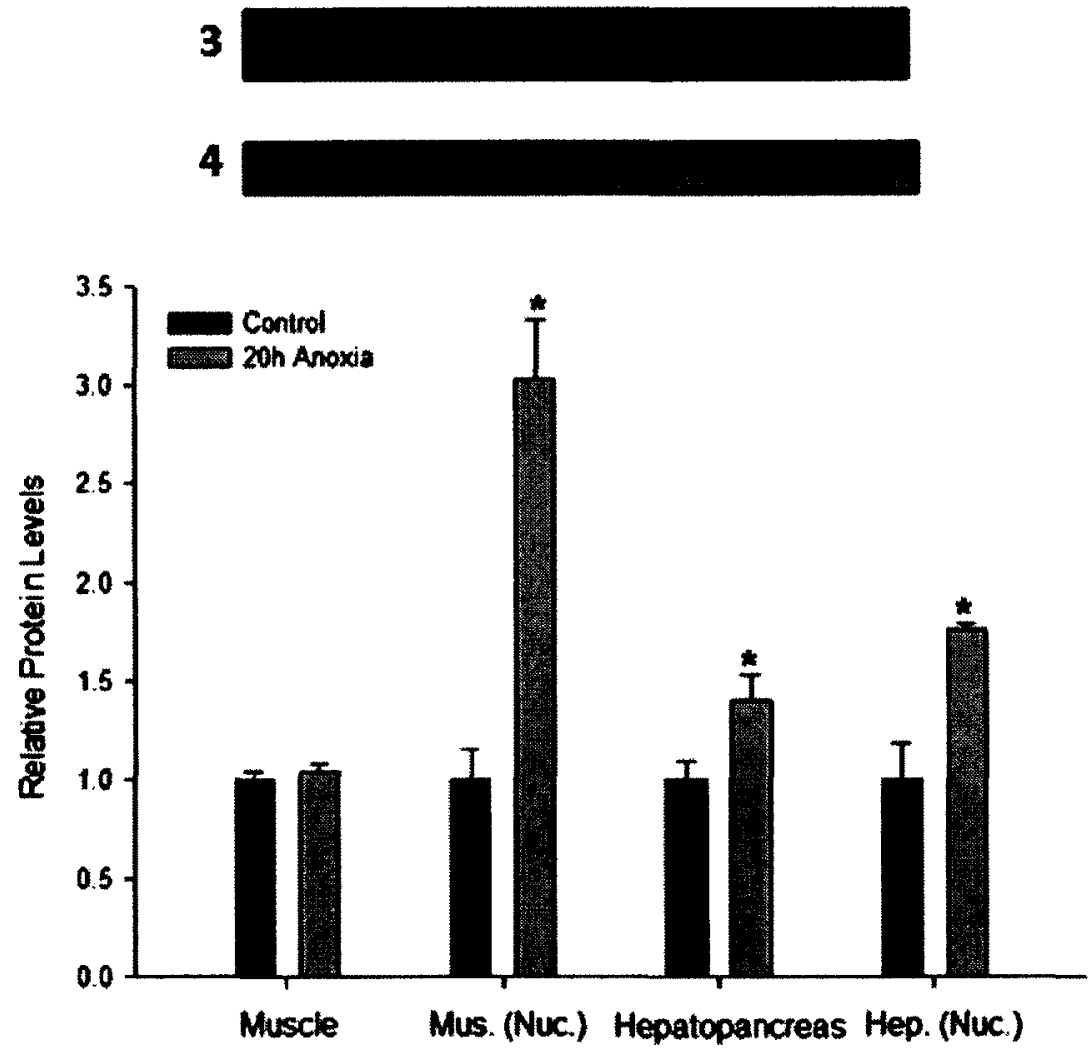

E2F-1

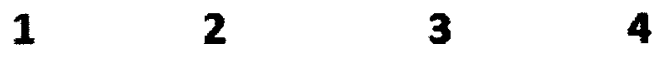

Figure 6.12: Effect of $20 \mathrm{~h}$ anoxia exposure on the protein expression and subcellular distribution of E2F-1 in $O$. virilis tissues.

Protein expression of E2F-1 in total soluble extracts and nuclear fractions of crayfish tissues (tail muscle, left; hepatopancreas, right) in response to anoxia, as determined by western immunoblotting.

Data are means $\pm S E M, n=4$ independent trials for muscle and hepatopancreas. * indicates significantly different from the corresponding control, $P<0.05$ 

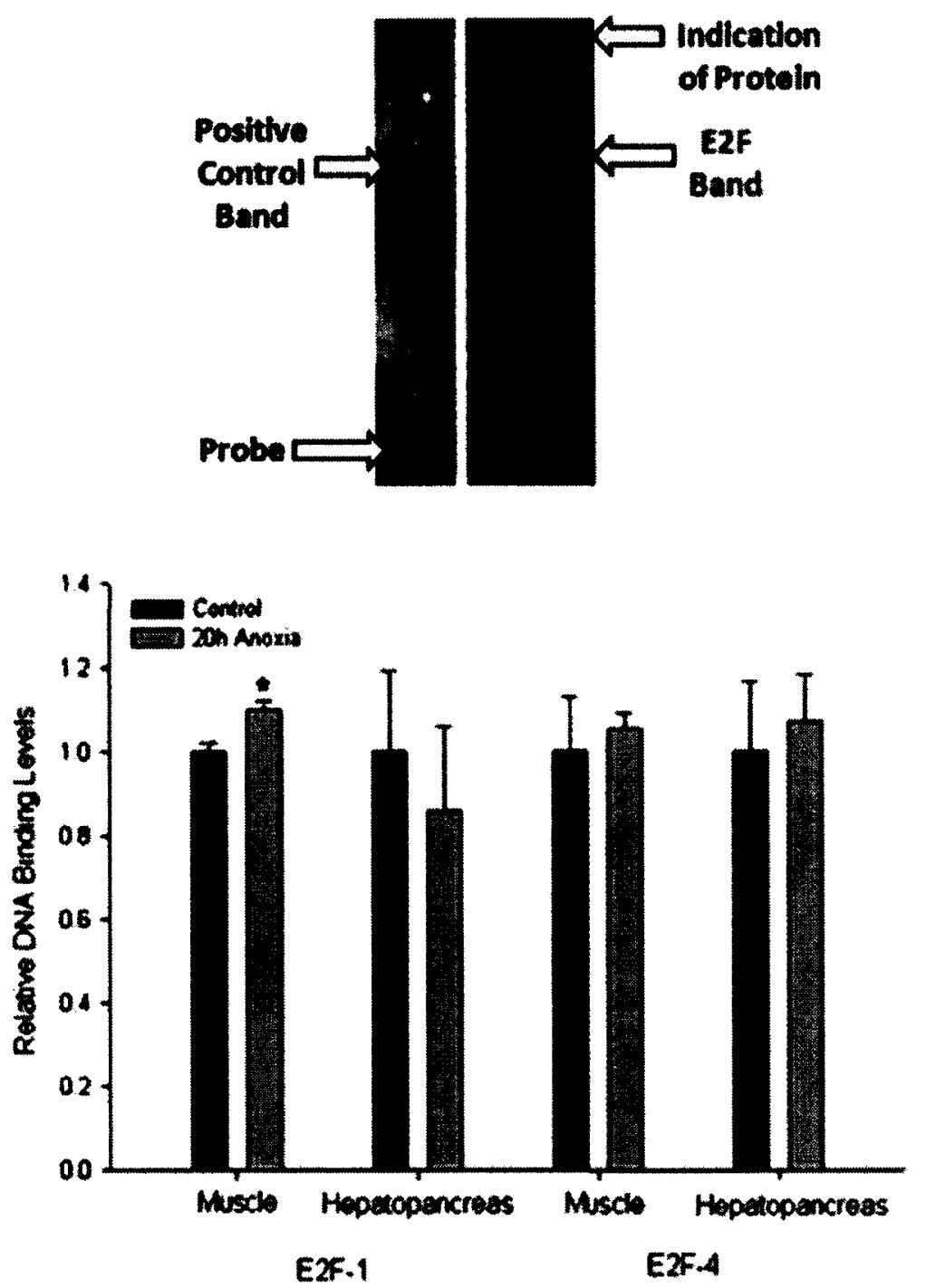

Figure 6.13: Effect of $20 \mathrm{~h}$ anoxia exposure on E2F (1 and 4) DNA binding activity in $O$. virilis tissues.

Changes in the DNA-binding activity of E2F-1 (left) and E2F-4 (right) in nuclear extracts of muscle and hepatopancreas in response to anoxia, as determined by a transcription factor binding assay. Data are means $\pm \mathrm{SEM}, n=4$ independent trials. * Indicates significantly different from the corresponding control, $P<0.05$.

Representative EMSA shown above the histogram. 

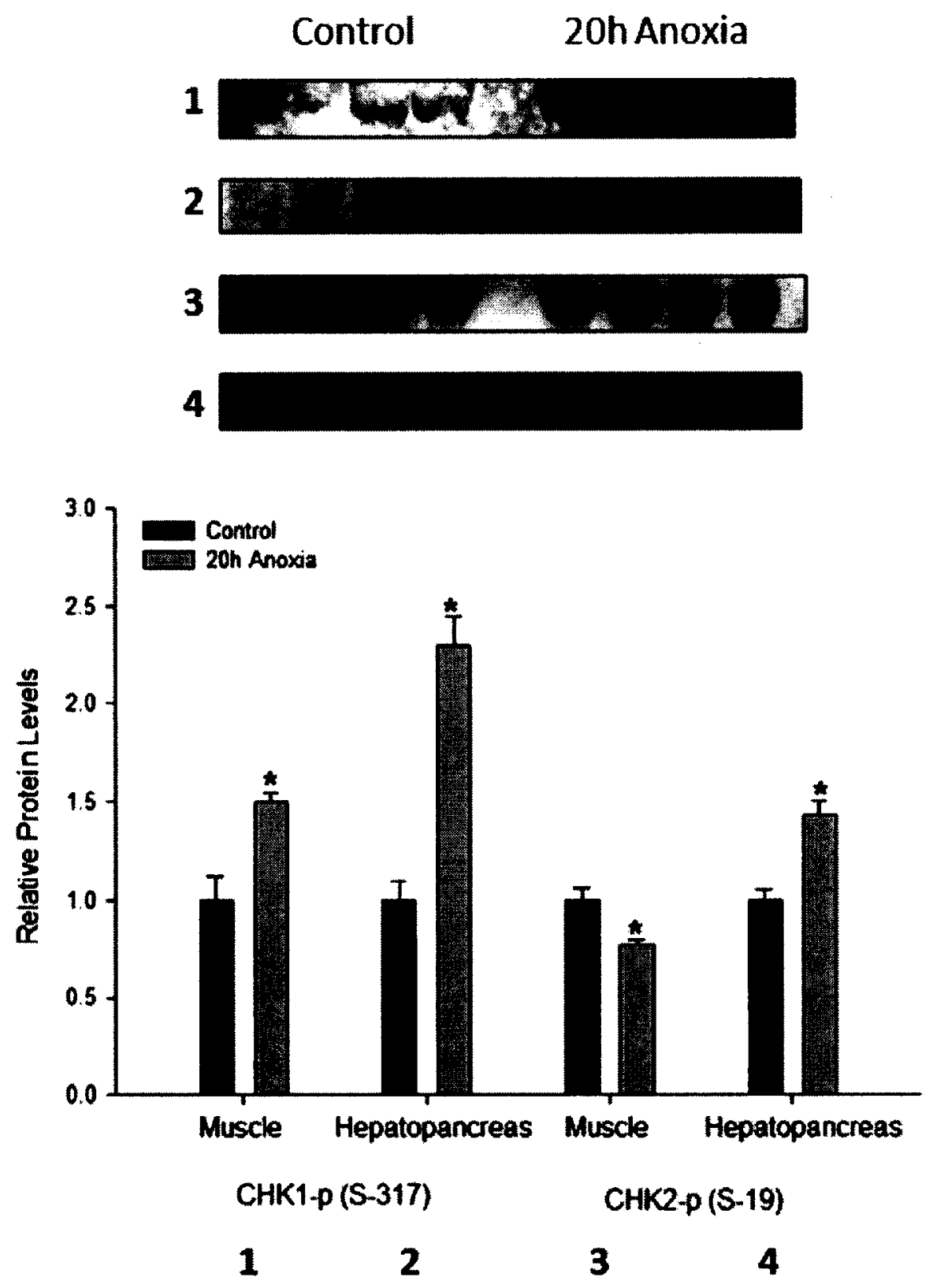

Figure 6.14: Effect of $20 \mathrm{~h}$ anoxia exposure on activation of Chk1 and 2 in $O$. virilis tissues.

Phosphorylation of Chk1 on serine 317 and Chk2 on serine 19 in total soluble extracts of crayfish tissues in response to anoxia, as determined by western immunoblotting. Data are means $\pm S E M, n=4$ independent trials for muscle and hepatopancreas. * indicates significantly different from the corresponding control, $P<0.05$. 


\section{Control 20h Anoxia}
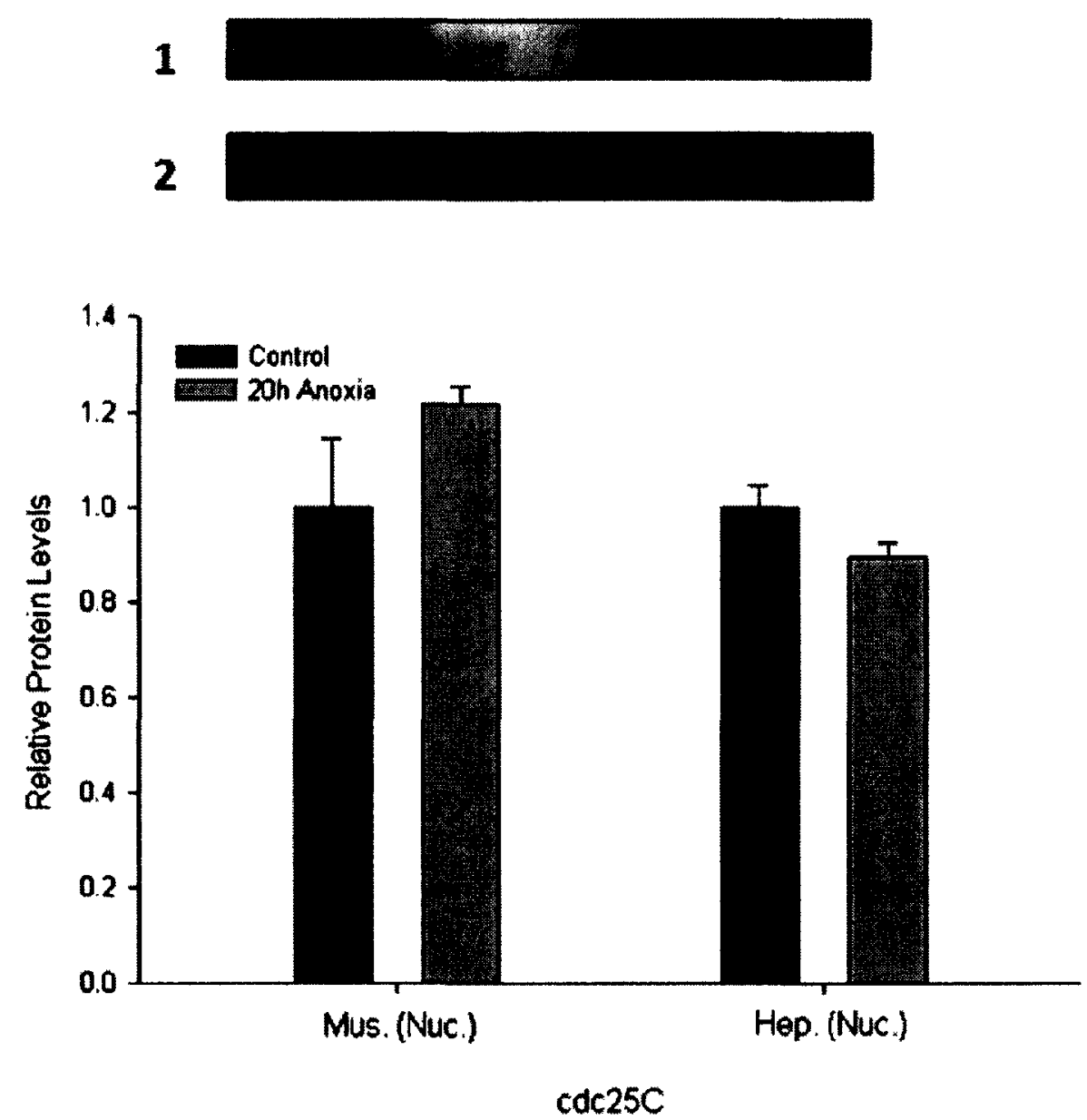

1

2

Figure 6.15: Effect of $20 \mathrm{~h}$ anoxia exposure on nuclear protein expression of cdc25C in 0 . virilis tissues.

Changes in relative protein expression of cdc25C in nuclear extracts of crayfish tissues in response to anoxia, as determined by western immunoblotting.

Data are means $\pm S E M, n=4$ independent trials for muscle and hepatopancreas. * indicates significantly different from the corresponding control, $P<0.05$. 


\section{Control 20h Anoxia}

1

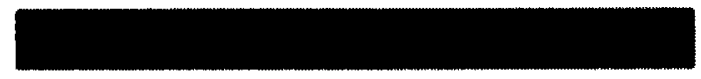

2

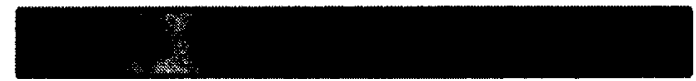

3

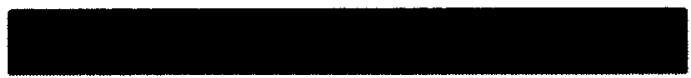

4
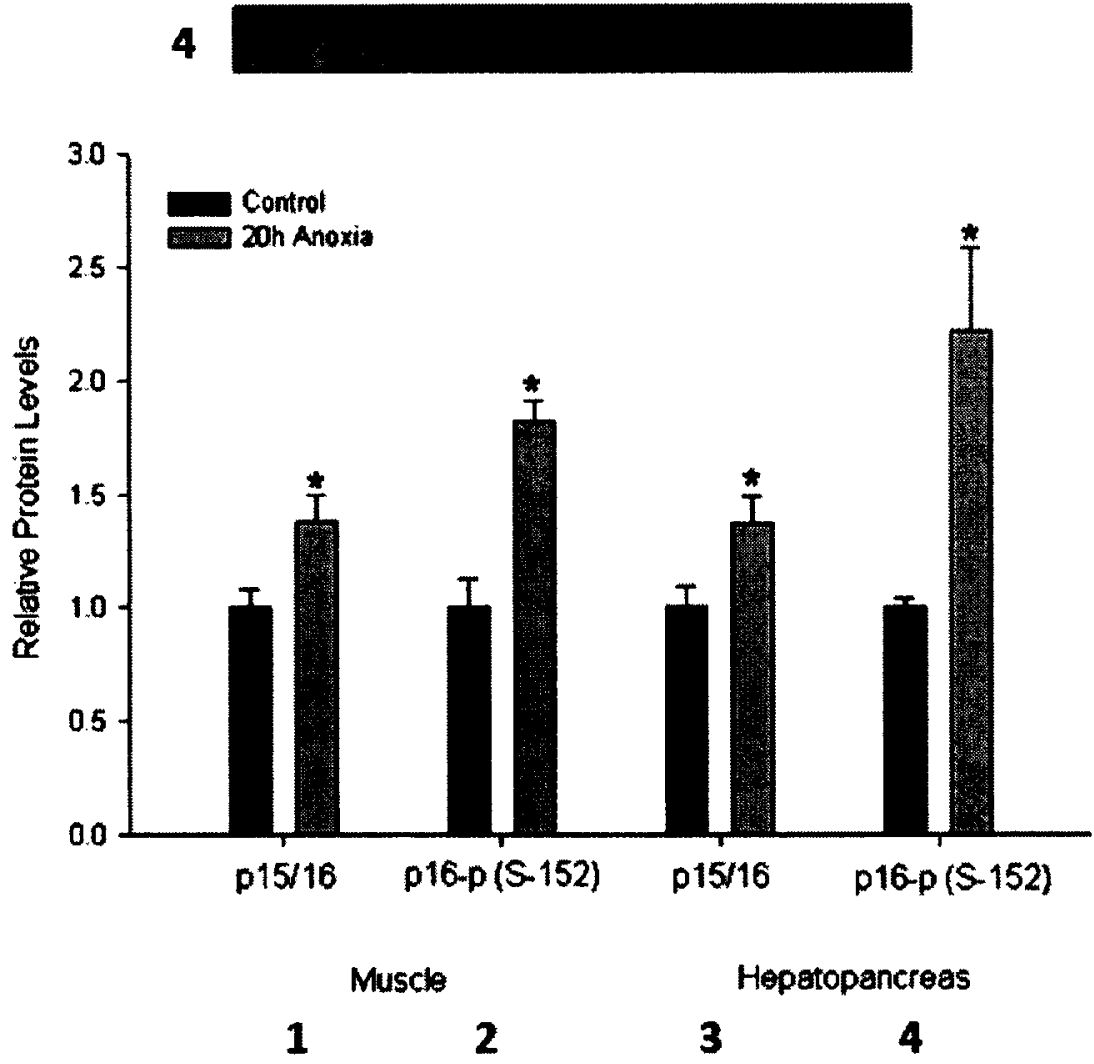

Figure 6.16: Effect of $20 \mathrm{~h}$ anoxia exposure on p15/16 protein levels and p16 activation in $O$. virilis tissues.

Changes in p15/16 protein levels and phosphorylation of p16 on serine 152 in total soluble protein extracts of crayfish tissues in response to anoxia, as determined by western immunoblotting.

Data are means \pm SEM, $n=4$ independent trials for muscle and hepatopancreas. * indicates significantly different from the corresponding control, $P<0.05$. 


\section{Control 20h Anoxia}

1

2
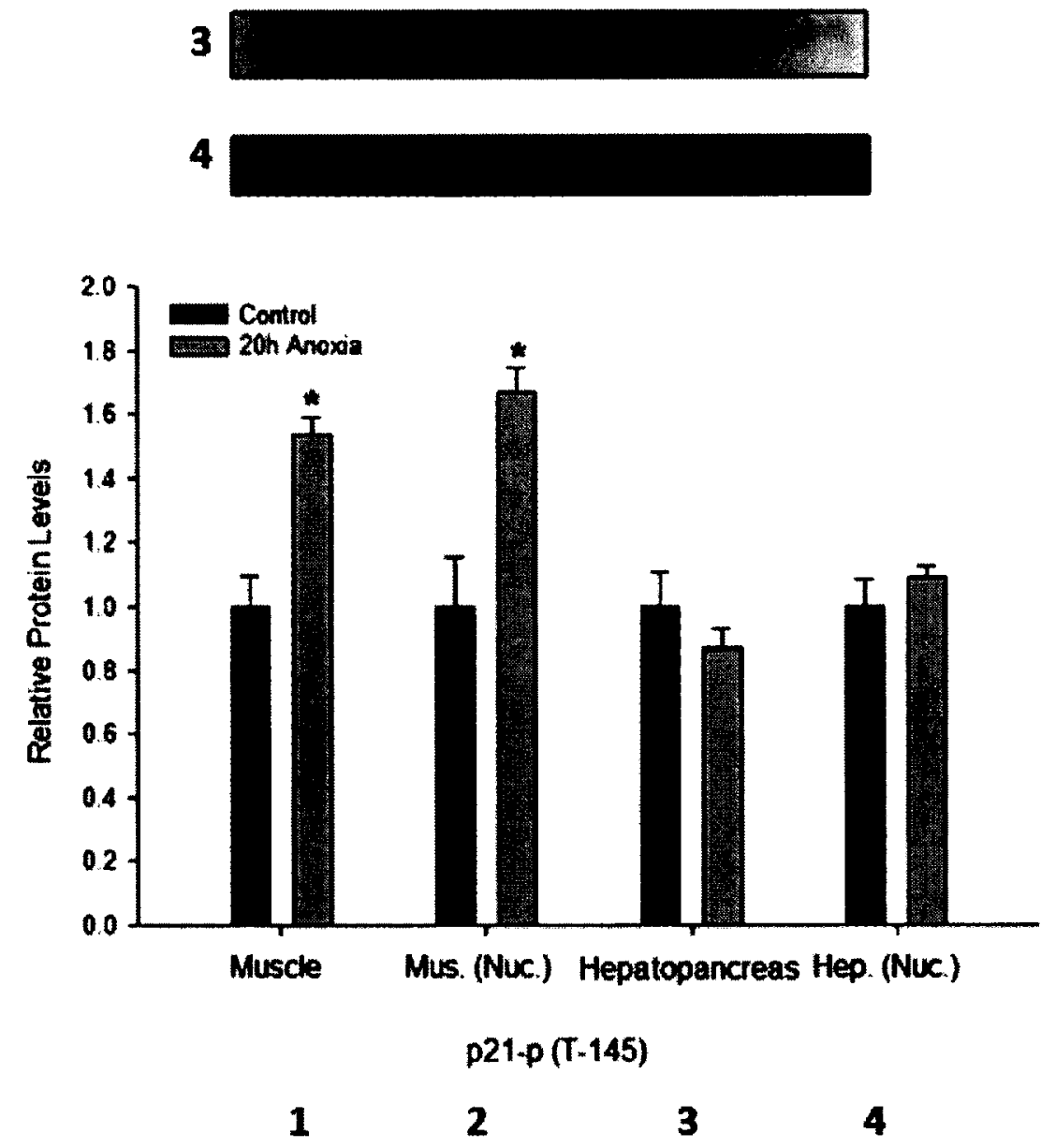

Figure 6.17: Effect of $20 \mathrm{~h}$ anoxia exposure on phospho-control and subcellular distribution of p21-p (T145) in $O$. virilis tissues.

Changes in $\mathrm{p} 21$ phosphorylation of threonine 145 in total soluble protein extracts and nuclear extracts of crayfish tissues in response to anoxia, as determined by western immunoblotting.

Data are means \pm SEM, $n=4$ independent trials for muscle and hepatopancreas. * indicates significantly different from the corresponding control, $P<0.05$. 
Control 20h Anoxia

1

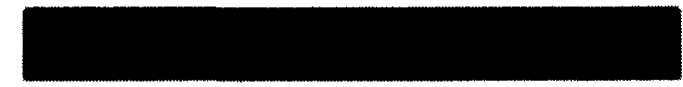

2
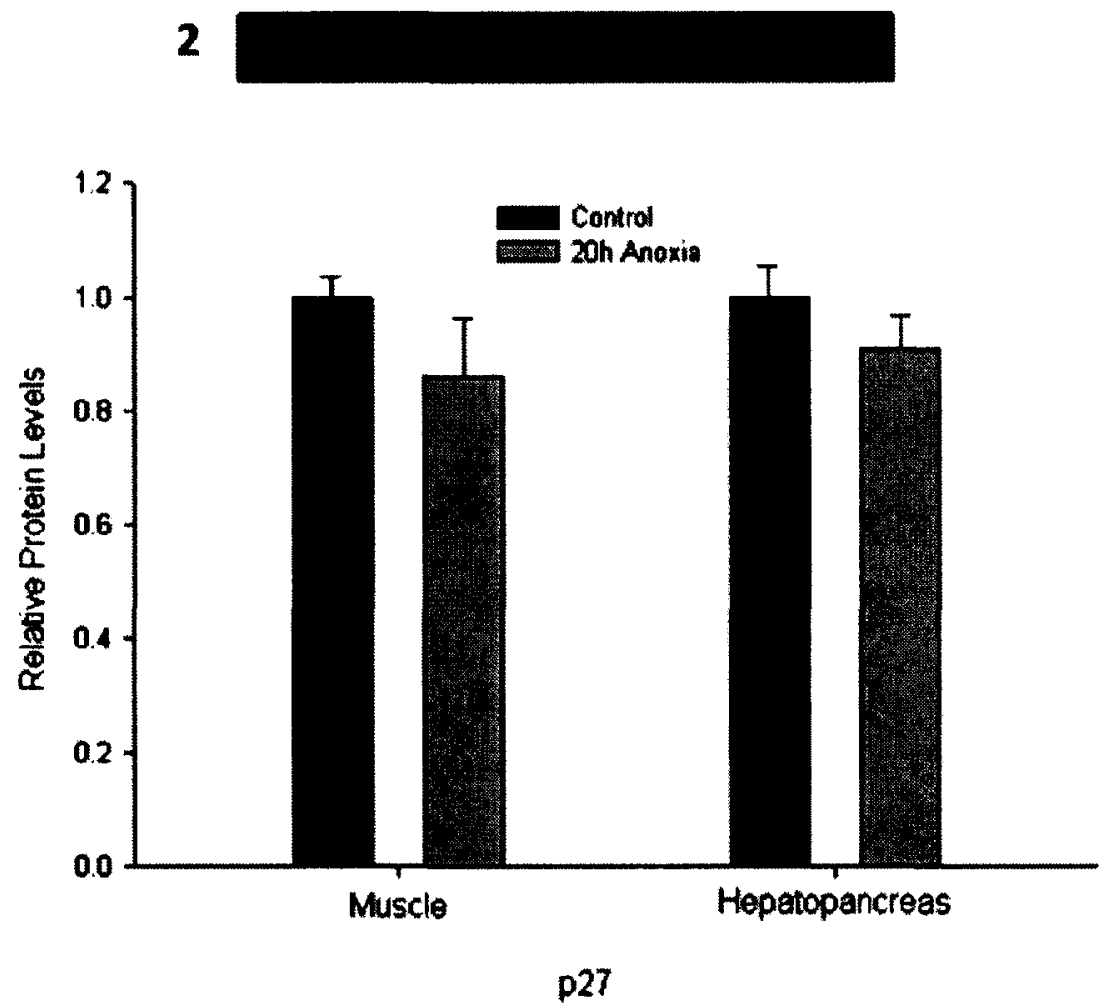

1

2

Figure 6.18: Effect of $20 \mathrm{~h}$ anoxia exposure on protein expression of $\mathrm{p} 27$ in $O$. virilis tissues.

Changes in relative protein expression of p27 in total soluble extracts of crayfish tissues in response to anoxia, as determined by western immunoblotting.

Data are means \pm SEM, $n=4$ independent trials for muscle and hepatopancreas. * indicates significantly different from the corresponding control, $P<0.05$. 
Table 6.3 Chapter 6 data summary

\begin{tabular}{|c|c|c|c|c|c|c|c|c|c|c|}
\hline \multirow[t]{3}{*}{ 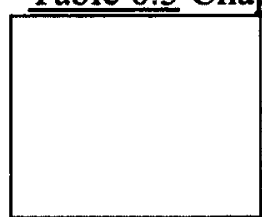 } & \multicolumn{2}{|c|}{$\begin{array}{c}\text { Protein } \\
\text { (Total) }\end{array}$} & \multicolumn{4}{|c|}{ Protein } & \multicolumn{2}{|c|}{ mRNA } & \multicolumn{2}{|c|}{ ELISA } \\
\hline & \multirow[t]{2}{*}{$\mathbf{M}$} & \multirow[t]{2}{*}{$\mathbf{H}$} & \multicolumn{2}{|c|}{$\mathbf{M}$} & \multicolumn{2}{|c|}{$\mathbf{H}$} & \multirow[t]{2}{*}{$\mathbf{M}$} & \multirow[t]{2}{*}{$\mathbf{H}$} & \multirow[t]{2}{*}{$\mathbf{M}$} & \multirow[t]{2}{*}{$\mathbf{H}$} \\
\hline & & & Cyto. & Nuc. & Cyto. & Nuc. & & & & \\
\hline CDK 2 & $\uparrow$ & $\downarrow$ & & & & & & & & \\
\hline CDK 4 & $\uparrow$ & $\uparrow$ & & & & & & & & \\
\hline CDK 6 & & & $\downarrow$ & $\uparrow$ & $\uparrow$ & $\uparrow$ & & & & \\
\hline Cyclin D & & & $\leftrightarrow$ & $\downarrow$ & $\uparrow$ & $\bar{\uparrow}$ & & & & \\
\hline Cyclin E & & & $\leftrightarrow$ & $\leftrightarrow$ & $\leftrightarrow$ & $\leftrightarrow$ & & & & \\
\hline Cyclin A & & & $\uparrow$ & $\leftrightarrow$ & $\uparrow$ & $\leftrightarrow$ & & & & \\
\hline Cyclin B & & & $\leftrightarrow$ & $\uparrow$ & $\uparrow$ & $\uparrow$ & & & & \\
\hline $\mathbf{R b}$ & & & & & & & $\uparrow$ & $\uparrow$ & & \\
\hline Rb-p (S780) & $\uparrow$ & $\uparrow$ & & & & & & & & \\
\hline E2F4 & $\leftrightarrow$ & $\leftrightarrow$ & & & & & & & $\leftrightarrow$ & $\leftrightarrow$ \\
\hline E2F1 & $\leftrightarrow$ & $\uparrow$ & & $\uparrow$ & & $\uparrow$ & & & $\uparrow$ & $\leftrightarrow$ \\
\hline $\begin{array}{l}\text { Chk 1-p } \\
\text { (S317) }\end{array}$ & $\uparrow$ & $\uparrow$ & & & & & & & & \\
\hline $\begin{array}{l}\text { Chk 2-p } \\
\text { (S19) }\end{array}$ & $\downarrow$ & $\uparrow$ & & & & & & & & \\
\hline cdc25C & & & & $\leftrightarrow$ & & $\leftrightarrow$ & & & & \\
\hline $\mathrm{p} 15 / 16$ & $\uparrow$ & $\bar{\uparrow}$ & & & & & & & & \\
\hline $\begin{array}{l}\text { p16-p } \\
\text { (S152) }\end{array}$ & $\uparrow$ & $\uparrow$ & & & & & & & & \\
\hline $\begin{array}{l}\text { p21-p } \\
\text { (T145) }\end{array}$ & $\uparrow$ & $\leftrightarrow$ & & $\uparrow$ & & $\leftrightarrow$ & & & & \\
\hline p27 & $\leftrightarrow$ & $\leftrightarrow$ & & & & & & & & \\
\hline
\end{tabular}

Note: Changes indicate statistical significance $(P<0.05)$; Westem Blotting $(n \geq 4)$, RT-PCR $(n \geq 4)$, ELISA $(\mathrm{n}=4)$

Arrows indicate changes or level profiles, in response to anoxia 


\section{Chapter 7 General Discussion}


Oxygen, the final electron acceptor in mitochondrial respiration, has allowed organisms to develop in size and complexity due to the vastly increased ATP production available from oxidative phosphorylation as compared with fermentative pathways. For animals reliant on aerobic metabolism, catabolism of most fuel sources depends on oxidative phosphorylation - and as such, the presence of oxygen. Anaerobic metabolism, on the other hand, produces only a fraction of the ATP yield that is produced in aerobic metabolism, is largely restricted to the use of carbohydrate fuels, and also acidifies cells as a result of proton accumulation. Hence, the challenge to survive using anaerobic metabolism alone is not well met by most types of animals. Despite this, there are a number of species and groups of animals that are adapted to survive prolonged exposures to severe hypoxic or anoxic conditions; some, like the red eared slider turtle (Trachemys scripta elegans), are even considered to be facultative anaerobes (Krivoruchko and Storey, 2010). Oxygen limitation or deprivation in animals may be the result of many things; altered oxygen solubility (aquatic animals), daily environmental changes (intertidal animals), altered ambient oxygen levels (burrowing or low mobility/high population animals), or as a result of adjustments to deal with other stressors (e.g. both dehydration and freezing stresses an impose oxygen limitation), to identify but a few.

For animals, dealing with the trials of acidification due to anaerobic metabolism is dependent on the particular model; some species produce alternative end-products to lactate that cause less acidosis (propionate/succinate; van den Thillart et al., 1992), others excrete anaerobic end products (e.g. ethanol in goldfish; Shoubridge and Hochachka, 1980) whereas others have mechanisms to buffer and store high levels of $\mathrm{H}^{+}$and lactate (turtle - Jackson et al., 2007; crayfish - Jackson et al., 2001). Oxygen deprivation is 
divided into two conditions; hypoxia (low $\mathrm{O}_{2}$ ) and anoxia (no $\mathrm{O}_{2}$ ). While some of the mechanisms are shared, hypoxia tolerance is more widespread than anoxia tolerance (or resistance) throughout phylogeny (Storey, 2004). Survival during oxygen deprivation is also dependent on the duration of exposure and, for ectotherms, the body temperature. While there are animals that endure daily exposures to short hypoxia exposures (diving animals; Hochachka, 1986), there are also animals that can undergo many months of hypoxia and/or full anoxia exposure (ex. turtles hibernating underwater; Storey and Storey, 2010). In terms of the responses to hypoxia, when exposure is short, changes are primarily made on the physiological level to respiration and cardiac activity to enhance oxygen uptake and delivery. With long term exposure to oxygen deprivation, significant changes to metabolism are necessary to regulate the major ATP-expensive cellular processes, typically involving both a strong global suppression of metabolic rate and a reprioritization of ATP use by energy-expensive processes so that those that are vital to survival are preserved. Regulation of gene expression, through transcriptional, translational and post-translational controls, is paramount to mediating the many changes in critical cellular pathways (Storey and Storey, 2004).

Physiologically, a number of crustaceans have been profiled in terms of their response to hypoxia. These species, including $O$. virilis, undergo changes to their oxygen uptake capacity through changes to their ventilation rates and hemocyanin oxygen affinities (McMahon, 2001). Cardiac output is also maintained through stroke volume manipulation (Reiber, 1995), in response to decreased muscular contraction associated with a reduced energy state. Less is known about molecular level responses to anoxia in crayfish although basic parameters of energy metabolism have been investigated in $O$. 
virilis and some other species (da Silva-Castiglioni et al., 2010). The $O$. virilis response to short term (1-4 h) anoxia includes a depletion of arginine phosphate (the first energy source to be used) as well as significant increases in ADP, AMP and lactate levels (K.B. Storey, data unpublished). This indicates that, in the short term, low oxygen challenge is dealt with by increased energy production from phosphagen catabolism and anaerobic glycolysis. The activities of protein kinases and phosphatases (PKA, PP1/2A/2C) also change quickly; PKA activity increases rapidly but then declines in concert with increased phosphatase activity (Cowan and Storey, 2001). This pattern suggests an initial PKA-mediated activation of glycogenolysis that is followed later when anoxia exposure is prolonged by suppression of PKA targets as the animals transition into a hypometabolic state. This is accompanied by a hypoxia/anoxia induced attenuation of glycolysis, via the regulation of 6-phosphofructokinase (PFK) activity. Changes in substrate (increased fructose-6-phosphate) and product (decreased fructose-1,6bisphosphate ) of PFK (K.B. Storey, data unpublished) indicate inhibition at this regulatory enzyme as do changes in the phosphorylation state of PFK (Cowan, 1998).

Phosphorylation regulation of glycolytic enzymes is well-known among animals that employ MRD to survive anoxia or other stresses. Indeed, in $O$. virilis, arginine kinase (AK) is also a target of reversible phosphorylation control under anoxic conditions. After 20 hours of anoxia exposure, AK showed a distinctly lower affinity for its substrate, Larginine, with the changes under anoxia linked to phosphorylation (potentially triggered by PKA) (Dawson and Storey, 2011). This confirms that posttranslational modification is a major stress response mechanism in crayfish as well as in other models of anoxia resistance/tolerance and MRD. As this thesis demonstrates, posttranslational modification 
by reversible phosphorylation also proves to be a powerful and widespread mechanism for coordinating the responses of many other cellular pathways and processes in response to the shift from aerobic to anaerobic metabolism.

In planning this thesis, a template was needed to identify potential targets for change under anoxia. This was provided by the model organism $C$. elegans which has its own hypometabolic period, the dauer stage. The mechanisms employed by C. elegans during entry to, and throughout dauer are critical and conserved mechanisms and represent core stress responses both in terms of the pathways regulated and the key protein/gene targets within them (see Appendix A). In this thesis, the comparable regulation of anoxia-induced MRD in crayfish was evaluated, using dauer targets as an outline to identify conservation (and also novel regulation) within the stress response. Three main areas were explored: developmental pathways, apoptosis and autophagy controls, and cell cycle regulation. The responses of $O$. virilis under anoxia (Tables 2.3, $3.3,4.3,5.2$ and 6.3 ) showed many similarities to those of $C$. elegans in dauer indicating that conserved mechanisms of MRD are widely used across phylogeny. Regulation of Akt signaling in $O$. virilis A critical step in C. elegans dauer entry is Akt repression, allowing activity of the downstream FoxO transcription factor (Daf-16) to transactivate numerous target genes for dauer. The suppression of canonical IGF (Daf-2)/PI3K (Age-1)/Akt (Akt-1) signaling is known to be the primary switch to dauer, and Daf-2 mutants are routinely used as dauer strains experimentally (Gems et al., 1998). In Chapter 2 of this thesis, the responses of the Akt signaling pathway to anoxia in crayfish were analyzed, and were found to be quite different from the C. elegans response. 
Upstream of Akt, the activating kinase (PDK1) was phosphorylated (and activated) in both tissues, with anoxia triggered increases in the tail muscle (FIG. 2.8). Consequently Akt was seen in its active, phospho-form under anoxia. Anoxia triggered increases expression of protein, along with a related increase in phosphorylation, in both tissues (FIG. 2.6). Conversely, the PTEN phosphatase which negatively regulates Akt phosphorylation, showed unchanged protein expression (FIG. 2.9). The ortholog of this phosphatase in C. elegans, Daf-18, is critical to dauer entry and exhibits increased activity (see Appendix A) (Fukuyama et al., 2006). Hence the crayfish model differs from C. elegans in this key aspect of MRD regulation.

Despite this, the regulation of downstream targets may indicate stress responsive cellular decisions. One of the most important aspects of Akt signaling, is that despite the pro-development responses it initiates, it is also a strong attenuator of apoptosis signaling. Both major downstream targets, GSK-3 and FoxO are implicated in cell cycle inhibition and subsequent apoptosis (Van Gorp et al., 2008; Liang and Slingerland, 2003; Gartel and Shchors, 2003). In O. virilis, despite an active Akt profile which would allow energy expensive procedures such as protein synthesis and glycogen synthesis, there is concurrent pro-survival signaling. Without a profile of cell cycle attenuation (Chapter 6 and Appendix A), an active Akt profile would conform to expectations. Akt can directly target cell cycle inhibitors (p21 and p27; Liang and Slingerland, 2003), and repress transcriptional activity of these same inhibitors through FoxO (direct transactivator) and GSK-3 (indirect activator; via c-myc). Indeed there were profiles of both GSK-3 and FoxO (FIG. 2.10, 15 and 16) phosphorylation on sites specifically targeted by Akt (and SGK, also activated, in the case of the FoxOs). Despite this, DNA binding activity by 
FoxO transcription factors increased (FIG. 2.17). This matches the dauer profile, but in the case of $O$. virilis may implicate an unknown nuclear mechanism (possibly a PTM), or decreased nuclear 14-3-3 expression (FIG. 2.22), under anoxia. The increased DNA binding activity was mirrored with an increase in MnSOD transcript levels; and subsequently, protein levels (FIG. 2.19, 20). Hence, while there was a general profile of FoxO repression, culminating in cytoplasmic anchoring of the transcription factors, a level of FoxO transcriptional activity was retained during anoxia. The accumulation of antioxidants, such as MnSOD, is a conserved mechanism of protection in anticipation of return to normal oxygen conditions and the rapid ROS generation that accompanies it, and has been seen in multiple models of MRD as a protective mechanism (Malik and Storey, 2011; Krivoruchko and Storey, 2010).

Overall, the data in Chapter 2 suggest a pattern of Akt activity that appears to function to prevent cell cycle attenuation and resultant apoptosis. However, despite this, increased antioxidant expression, likely transactivated through FoxO activity, indicates that non-canonical Akt activities are also in place. Hence, while the hypothesis of Akt activation for pro-survival mechanisms holds, in comparison to the key $C$. elegans mechanism for dauer entry, the crayfish $O$. virilis is not completely the same; although this is likely related to the differences in stringency of the hypometabolic periods and the lack of cell cycle attenuation in the crayfish.

Regulation of Smad/STAT activities in $O$. virilis under anoxia

Chapter 3 assessed another group of pro-development proteins, in the Smad and STAT families of transcription factors. Both groups are strongly linked with standard developmental responses, and are highly active in periods of organismal growth 
(Massagué et al., 2005; Watanabe and Arai, 1996). The Smads are the main effectors of the TGF- $\beta$ signaling pathway that is similar to the insulin/IGF pathway in being a membrane receptor based path responsive to growth factors. In $C$. elegans the TGF- $\beta$ pathway is turned off during dauer, in parallel to Akt signaling, and this prevents the prodevelopment Smads transcription factors from functioning throughout dauer (see Appendix A and B.3/4) (Inoue and Thomas, 2000). There are both pro- and anti- dauer Smads in C. elegans, with the anti-dauer members working upstream of the pro-dauer members, and inhibiting their nuclear activity under normal conditions (Beall and Pearce, 2002). Smads require both phosphorylation and complex formation to have transcriptional activity, and in $O$. virilis hepatopancreas, there were phosphorylation events triggered by anoxia (FIG. 3.3, 4), but no discernible increases in nuclear Smad 2, the 'main' Smad (FIG. 3.5). Smad 3 transcript levels, in conjunction with protein levels increased in the muscle (FIG. 3.7, 8), but only showed increased DNA binding activities in the hepatopancreas (FIG. 3.9). Similarly, there were no changes in protein levels of Smad 4, the transporting Smad (FIG. 3.10). While this presents a tenuous profile of increased Smad activity, again contrary to the dauer response, there is little to suggest significantly increased transcriptional activity; particularly in the tail muscle, in terms of nuclear accumulation and DNA binding activity. One of the potential explanations for this is the role that nuclear Smad co-activators play; their absence (or binding to other proteins) would likely present a significant control mechanism. The hepatopancreas, a tissue that has a rapid cell cycle under normal conditions, must surely slow down based on energetic limitations under anoxia, but this would still amount to a level of development seen even under anoxic conditions. Hence, the phospho-profile of Smads 
(1/5 and 2) along with DNA binding of Smad 3, accords somewhat with the requirements of the tissue.

The JAK/STAT pathway is similarly signaled by cytokines and growth factors, at tyrosine kinase membrane bound receptors. In C. elegans the single STAT ortholog, STA-1 is active in parallel (particularly in tissue expression) with TGF- $\beta$ signals. While the role of STA-1 is classified as anti-dauer, it is active throughout the dauer period (Wang and Levy, 2006). In other animals, there are multiple STAT members, and in some cases activity is complementary (STAT1/2; Bluyssen and Levy, 1997) and in other cases antagonistic (STAT 1 and 3; Stephanou and Latchman, 2003). The STATs are activated through phosphorylation, and in $O$. virilis while there was an increase in STATl phosphorylation (FIG. 3.13), there appeared to be reduced activation in the nuclear fractions (of both tissues) under anoxia. Subsequently, there was even decreased DNA binding under anoxia in the hepatopancreas (FIG. 3.14). This may have less to do with a role in development, than with the STAT1 ability to induce apoptosis; transcriptionally inhibiting anti-apoptosis targets, activating pro-apoptosis targets, and combining with the tumor suppressor p53 to enhance its activity (Stephanou and Latchman, 2003). STAT2, which can complex with STAT1, also showed an anoxia linked partial decrease in phosphorylation (FIG. 3.15), while the anti-apoptotic STAT3 (which shares apoptosis related gene targets with STAT1) showed increased phosphorylation in the tail muscle (FIG. 3.16). This may represent a pro-survival decision, under anoxia, to prevent STAT1 activity, via STAT nuclear inactivity and STAT2 phospho status, while allowing STAT3 activity. 
In Chapter 3, therefore, what was seen is that despite apparent patterns of activation (Table 3.3), the Smad and STAT families of transcription factors are likely not induced beyond control levels under anoxia. This, largely holds with the hypothesis, and even parallels to aspects of dauer stage regulation.

There is some evidence of decreased activity, particularly focusing on nuclear phosphorylation profiles of key members, but on the whole the transcription factors may be functioning at control levels. The build-up of phospho-Smads may even suggest a preparative response of the cells, for recovery of the cells, post anoxia. This, in line with antioxidant accumulation and autophagy profiles, may explain activated, but inactive prodevelopmental transcription factors, which would certainly fit a requirement of rebuilding following a prolonged stress period. Similarly, STAT1 shows activation (via phosphorylation) without relatively increased activity (nuclear profiling and DNA binding). This may be due to its role in apoptosis signaling, an obvious process to inhibit especially during anoxia, but may also be a preparatory response. The activation of STAT3 and inactivation of STAT 2 suggests the former, but in line with the Smad profile, preparing for cell development and differentiation might also be expected. Regulating apoptosis signaling through $p 53$ activity, in $O$. virilis Chapter 4 analyzes one of the key apoptosis signaling molecules in cells - the p53 tumor suppressor. P53 is linked with multiple roles in cellular death (Rinn and Huarte, 2011), along with novel roles as a nuclear interactor (TGF- $\beta$ signaling; Atfi and Baron, 2008) and even cellular survival (autophagy; Crighton et al., 2007). In C. elegans, the p53 ortholog (Cep-1) holds the same role as a DNA damage induced cell death activator. Ectopic apoptosis, in somatic tissues, is poorly dealt with in C. elegans, and can result in 
the death of the nematode if left unchecked (Arum and Johnson, 2007). While Cep-1 knockout leads to increased life-span, Cep-1 inactivation does not alter dauer entry or Daf-16 (FoxO) nuclear localization (Arum and Johnson, 2007). In O. virilis there was a distinct profile of p53 activity (Table 4.3); from increased activation of upstream DNA damage activators (ATM/ATR; FIG. 4.2), to reduced expression of the p53 MDM2 inhibitor (FIG. 4.10). Given these results, p53 phosphorylation was predicted, and indeed phosphorylation on serine residues (15; FIG. 4.4 and 37; FIG. 4.9) was evident in response to anoxia. Despite this, nuclear phosphorylated p53 was only increased in the tail muscle (FIG. 4.5). This correlated with increased DNA binding (FIG. 4.6), and increased transcription of a p53 target (EI24; FIG 4.8) in the tail muscle. Conversely, in the hepatopancreas, phospho-p53 in nuclear fractions remained static, but DNA binding in fact decreased. This relates more closely to the $C$. elegans model, but also illustrates a potentially tissue specific response.

Potential apoptosis signaling in a tissue critical for whole organism survival (such as the hepatopancreas), would be highly unlikely, especially in a stress resistant model. Indeed while the hypothesis predicted reduced p 53 activity for reduced apoptosis, p 53 activity in relation to autophagy may hold with the pro-survival concept of the hypothesis. Hence, in the tail muscle, p53 activity may be indicative a novel pro-survival role. The first line of reasoning is that apoptosis through DNA damage would be unlikely given the lack of oxidative damage during anoxia, and secondly, with survival through anoxia, there is very little apoptosis. This would not be true in a traditional profile of stress induced p53 activity. Given the results in Chapter 5, there was a proautophagy/anti-apoptosis profile in the tail muscle, potentially linking the two responses. 
Recently, it has been discovered that despite what was thought to be an antagonistic or competitive relationship between the induction of autophagy and apoptosis, p53 is able to induce both. Through transactivation of TOR inhibitors (Balaburski et al., 2010), which would relieve TOR repression of autophagy, along with DRAM transactivation (Crighton et al., 2007), p53 has a potentially pro-autophagy mechanism. Hence, under anoxia in $O$. virilis tail muscle, $\mathrm{p} 53$ signaling may actually contribute to cellular survival; albeit through cellular minimization (autophagy) which maintains energetic homeostasis.

Determining the fate of the cell; apoptosis vs. autophagy signaling

Chapter 5 looked at the mechanisms that determine cellular fate, under prolonged stress exposure. Both apoptosis and autophagy have roles in quality control in unstressed cells, when expressed at basal levels. Stress induced apoptosis, or autophagy, however, are much more serious prospects, with regards to cellular survival. Apoptosis, particularly signaled as part of a DNA damage response, results in cellular death, while autophagy confers cellular survival (and minimization) under low energy states. Apoptosis is particularly dangerous, with the capacity to trigger a cascade that spreads from the death of one cell to its surrounding cells, leading to tissue or organ damage and potentially death of the whole organism. Indeed, while there is little oxidative stress apparent in a period of oxygen limitation, apoptosis can be triggered by a number of environmental stress conditions including hypoxia (Malhotra et al., 2001), nucleotide deficiencies (Menze et al., 2010) and freezing (Yi and Lee, 2011).

One of the most critical markers of apoptosis signaling is the formation of the outer mitochondrial membrane pore (OMMP). Regulating the stability of the membrane 
are the anti-apoptotic members of the $\mathrm{Bcl} 2$ subfamily (Bcl-2 and $\mathrm{Bcl}-\mathrm{xl})$, which belong to the pro-survival (oncogenic) $\mathrm{Bcl}$ family of proteins. In C. elegans the $\mathrm{Bcl}-\mathrm{xl}$ homolog (CED-9) binds a pro-apoptotic protein (APAF1; apoptotic protease activating factor 1), preventing pore formation (Samara and Tavernarakis, 2008). Concurrently, the egg laying abnormal (EGL-1) protein, which has the critical $\mathrm{BH} 3$ domain that Bcl proteins use for interactions, also localizes to the mitochondrial surface to trigger pore formation (Samara and Tavernarakis, 2008). In terms of regulation during the dauer period, overexpression of apoptosis would undoubtedly result in severely damaged worms, and indeed, the necessity for anti-apoptotic gene function in dauer is shown via the requirement for the CED-9 positive interacting protein MISC-1, which suppresses dauer if absent (Gallo et al., 2011). Under anoxia in O. virilis the same approximate mechanism was seen, albeit in a tissue specific manner (Table 5.2). As with p53 expression, the significant increases in Bcl protein expression (FIG. 5.3, 4) and activity through phosphorylation (FIG. 5.3) occurred only in tail muscle. In the hepatopancreas, Bcl profiling showed a distinct reduction. This, of course, was not in conjunction with increased apoptosis, but potentially indicates that there is less pro-apoptotic signaling to mediate. On the other hand, muscle tissue, is less crucial to whole animal survival through a stress period and may be compromised energetically; as a result having levels of apoptosis signaling in need of suppression. This tissue specific profile also extended to the expression of autophagy.

Autophagy is triggered by the co-ordinated activities of autophagy proteins (Atgs), and elevated protein levels of certain Atgs are markers of starvation induced autophagy (Huang et al., 2000). In C. elegans during dauer, autophagy is a critical 
mechanism to overcome energetic limitations. Caloric restriction, being one of the primary initiators of the dauer stage, results in autophagy up-regulation. The presence of autophagy proteins are not necessary for dauer entry, but specific deletions result in altered dauer transitions, and even death during the hypometabolic period (Kourtis and Tavernarakis, 2009). Similar to Bcl profiling, $O$. virilis tail muscle showed an analogous response to the C. elegans profile (see Appendix A); which incidentally, unlike hepatopancreas, more closely fulfills the hypothesis of anti-apoptosis/pro-autophagy signaling.

Protein increases of Beclin-1, Atg12, 7, 8 (FIG. 5.5, 6, 7, 8) and the cleaved form of Atg8 suggested a positive profile for induced autophagy under anoxia in the muscle. The hepatopancreas, as with $\mathrm{Bcl}$ signaling, indicated the opposite. These results were also supported by the profile of the major autophagy regulator, TOR kinase. TOR kinase is a negative regulator of autophagy, and in C. elegans the drop in activity of this prodevelopmental kinase is correlated with increased autophagy (Morck and Pilon, 2007). Indeed in $O$. virilis tail muscle, TOR complex (TORC) activity, through expression of TOR and G $\beta L$ (a complex component), along with activity of the TOR inhibitor (PRAS40), and TOR target (p70S6K) (FIG. 5.9, 10, 11), remained level; and is even suggestive of decreased activity. Conversely, in the hepatopancreas, anoxia appears to induce increased TORC activity (through the same profile). A TORC based prodevelopment response to anoxia in the hepatopancreas is congruous with Akt and Smad profiling, and similarly, the tail muscle TORC profile corresponds with autophagy and p53 expressions. 
Overall, there appears to be a strong profile for a tissue specific apoptosis/autophagy response (especially in conjunction with chapter 4). The tail muscle, which shows a more 'dauer' like profile in this respect, may be subject to autophagy in order to maintain overall energetic homeostasis. Autophagy minimizes cells, by removing fractions of the cytoplasm containing cellular components that are broken down and recycled for energy production. This both reduces the energetic needs of the target cell, and can also provide precursors for other cells. In terms of organ importance, skeletal muscle may be more expendable, comparable to the hepatopancreas, and as such, under prolonged stress exposure, autophagy may be the solution.

Controlling the cell cycle, under anoxia

In chapter 6, cell cycle components and their major regulators were analyzed in order to assess the state of cell cycle progression in crayfish tissues under anoxia stress. Cell cycle progression and cellular differentiation is a major energetic expenditure. In numerous models, cell cycle attenuation, and entrance into quiescence is the response to stress conditions; this includes oxygen deprivation. In $C$. elegans, cell cycle attenuation occurs throughout the dauer phase with multiple signals preventing cell cycle progression. The mechanism of $C$. elegans cell cycle regulation is conserved throughout phylogeny, with the same major regulation points at the $\mathrm{G}_{1} / \mathrm{S}$ and $\mathrm{G}_{2} / \mathrm{M}$ barriers (Van den Heuvel, 2005). Likewise, the components of the $C$. elegans cell cycle, though less numerous than their counterparts in other animal models, are essentially the same (see Appendix A), with cyclin/CDKs targeting $\mathrm{Rb}$ (LIN-35) as the controlling mechanism through $\mathrm{G}_{1}$ and into $\mathrm{S}$. The cyclin/CDKs are also regulated by cell cycle inhibitors (CKI-1) and phosphorylation state (WEE-1.3 and CDC-25.1) (Van den Heuvel, 2005). At the $\mathrm{G}_{2}$ barrier, cyclin 
$\mathrm{A} / \mathrm{B} / \mathrm{CDK} 1$ like complexes are also needed, further indicating conservation of the cell cycle.

In $O$. virilis, the overwhelming outlook is one of cell cycle progression (Table 6.3), albeit with some potential deceleration indicated by certain inhibitor profiles. At the $\mathrm{G}_{1} / \mathrm{S}$ barrier, there was increased phosphorylation of $\mathrm{Rb}$ (potentially linked to increased transcript levels) (FIG. 6.9, 10) in both tissues, coupled with increased nuclear presence of the E2F-1 transcription factor (FIG. 6.12). Despite this increase, only the tail muscle showed an anoxia linked increase in E2F-1 DNA binding activity (FIG. 6.13). This correlates with a largely up-regulated cell cycle profile in the tail muscle during anoxia, with increased phosphorylation (inactivation) of the p21 cyclin inhibiting protein (FIG. 6.17), and decreased Chk2 (activation) phosphorylation (FIG. 6.14). In the hepatopancreas, while there were elements suggesting progression through $G_{1}$ (such as $\mathrm{Rb}$ phosphorylation, increased nuclear cyclin $\mathrm{D}$ and accumulation of E2F-1 in the nucleus), there was no change in E2F-1 DNA binding activity. This is a crucial point for entry into the S phase, and while 'no-change' still indicates E2F-1 activity, the cell cycle is probably functioning at a regular if not lower rate. This is also supported by phosphorylation (and activation) of both checkpoint kinases, as well as a 'control' profile of p21 activity. What is also interesting, in both tissues, is that despite showing traits for an active cell cycle, the p27 kinase inhibiting protein expression was at control levels under anoxia (FIG. 6.18), and p16 protein levels and activation were increased under anoxia (FIG. 6.16). Both inhibitors affect the kinases at the $G_{I} / S$ transition, and it is important to note that while there was certainly progression signals, inhibitor expression should not be underestimated; it may be the single most important regulatory factor in $C$. 
elegans quiescence (Fukuyama et al., 2003).

At the $\mathrm{G}_{2} / \mathrm{M}$ barrier, expression profiles of the critical cyclins (A and B; FIG. 6.6, 7) showed a mixed pattern of cell cycle progression, triggered by anoxia. Cyclin A levels rose in the cytoplasmic fractions of both tissues. With cyclin A needing nuclear localization to be active (nuclear levels remain static with anoxia), the accumulation in the cytoplasm may suggest decreased nuclear shuttling; which would not enhance mitotic entry. However, increases in relative cyclin B protein levels, in nuclear fractions of both tissues under anoxia were a strong signal for mitosis given the importance of cyclin B (complexed with $\mathrm{CDK} 1$ ) at this juncture. In terms of the regulatory mechanisms at this barrier, the checkpoint kinases are important in their negative regulation of $\operatorname{cdc} 25 \mathrm{C}$, a cylinB/CDK1 activator. $\mathrm{Cdc} 25 \mathrm{C}$ undergoes nuclear translocation in order to maintain the correct phospho-state of cyclin $\mathrm{B} / \mathrm{CDK} 1$, which itself must be in the nucleus to be functional. Despite largely active Chk 1 and 2 profiles, nuclear levels of cdc25C remained unchanged from controls under anoxia.

And so, overall, the snapshot of the cell cycle after 20 hours of anoxic exposure is one of relative activity with potential for abatement, if not full attenuation. While this does not fulfill the prediction of the hypothesis, it holds with the rest of the crayfish data which shows maintained developmental responses during anoxia. Indeed, it appears that as part of its anoxia resistance, $O$. virilis does not have to enter a severely restricted state, in this case quiescence, in order to survive.

\section{Conclusions}

In summary, while the template of stress response from the $C$. elegans dauer state was a useful guide and illustrated interesting and previously unstudied pathways in $O$. virilis, it 
became apparent that the two forms of stress-induced metabolic challenges did not show a high degree of conservation in their molecular regulation. This may be largely due to the stringency of the $C$. elegans dauer period, in comparison to the more occasional or seasonal stress exposures of the crayfish. The dauer period employs severe restrictions on its cells to essentially make them non-aging cells. Attenuation of the cell cycle outlines the necessary activities of important tumor suppression proteins, which in turn determines the activities of many other critical proteins in a number of pathways. The non-linear effect of this is the correlated suppression of any proteins that may be considered to be pro-survival/proliferation. The role of autophagy in C. elegans can also not be understated, with the control of autophagy activity also able to potentially limit apoptosis signaling. The duration of the dauer period is also significantly longer than the combined span of all of its larval and mature adult stages; often up to 10 times as long as total lifespan. In contrast, the crayfish may (or may not) experience environmental hypoxia/anoxia at random and variable times within its normal lifespan of approximately three years. Its capacity for stress resistance and the necessity of stress resistance are not linked to crayfish developmental state, and as such the limitations on development and differentiation are not in place, as with the nematode. $O$. virilis appears to mediate stress through a combination of pro-survival signals (indeed some specifically targeted against cell cycle attenuation - which can induce apoptosis) and preparative responses. It may be that, in terms of cellular workload and energy usage:availability ratios, the recovery period is more taxing on the crayfish (and capable of causing more damage) than the stress period itself. Nonetheless, what is apparently conserved is the importance of prevention of apoptosis, through regulation of critical proteins, and support of autophagy. 
Both organisms are hundreds of millions of years old, and as such have had a very long time to specialize and adapt. Nonetheless, many of the mechanisms and components that are critical to survival and development are shared, and conserved throughout phylogeny up to humans. The stress resistance mechanisms of crayfish, although different in a number of ways from those of $C$. elegans, show tremendous potential as models for natural MRD, particularly in relation to oxygen deprivation based disease studies, and as such should be continued and expanded in the future. 
APPENDICES 


\section{Appendix A: Trends in C. elegans Dauer Response}

\begin{tabular}{|c|c|}
\hline Pathway/Key Target & Dauer Trend (Activity) \\
\hline \multicolumn{2}{|r|}{ Chapter 2} \\
\hline Akt pathway (Total) & Reduction \\
\hline Receptor signaling & Reduced \\
\hline PDK1 & Reduced \\
\hline Akt & Reduced \\
\hline PTEN & Enhanced \\
\hline GSK 3 & Enhanced \\
\hline FoxO & Enhanced \\
\hline SOD & Enhanced \\
\hline \multicolumn{2}{|r|}{ Chapter 3} \\
\hline TGF- $\beta$ signaling (Total) & Reduction \\
\hline Receptor signaling & Reduced \\
\hline Pro-development Smads & Reduced \\
\hline Pro-dauer Smads & Enhanced \\
\hline STAT signaling (Total) & Level \\
\hline STAT & Level \\
\hline \multicolumn{2}{|r|}{ Chapter 4} \\
\hline p53 signaling (Total) & $\begin{array}{l}\text { Lifespan extension (not related to dauer } \\
\text { induction) }\end{array}$ \\
\hline \multicolumn{2}{|r|}{ Chapter 5} \\
\hline Apoptosis (Total) & Decrease \\
\hline Bcl-xl activator & Enhanced \\
\hline Autophagy (Total) & Increase \\
\hline Beclin 1 & Enhanced \\
\hline $\operatorname{Atg} 7$ & Enhanced \\
\hline Atg 8 & Enhanced \\
\hline TORC activity & Reduction \\
\hline TOR & reduced \\
\hline Raptor & reduced \\
\hline \multicolumn{2}{|r|}{ Chapter 6} \\
\hline $\begin{array}{l}\text { Cell Cycle regulation } \\
\text { (Total) }\end{array}$ & Attenuation \\
\hline Cell cycle inhibitors & Enhanced \\
\hline Cyclins & Reduced \\
\hline CDKs & Reduced \\
\hline $\mathrm{Rb}$ & Enhanced \\
\hline Wee Kinase & Enhanced \\
\hline
\end{tabular}




\section{Appendix B: C. elegans Descriptions}

1. When conditions are particularly difficult, the worms may enter a period of stasis, postponing entry into a mature larval stage, and re-enter development when the conditions have become more favourable (Vanfleteren and Braeckman, 1999). The dauer period is critically controlled by its molecular signaling, particularly through the expression of 'abnormal dauer formation' (Daf) genes (Beall and Pearce, 2002). In entry to and maintenance of dauer larvae there are three main processes that are targeted; development, apoptosis/autophagy and the cell cycle. In order to enter the dauer stage, pro-developmental pathways must first be abated. The canonical pathway of protein kinase B (Akt) signaling is the key switch in this regard; inhibiting its activity allows the activity of pro-dauer transcription factors which are critical to dauer entry (Gems et al., 1998). In parallel, the TGF- $\beta$ developmental response is also abated (Beall and Pearce, 2002). Regulation of cell death is also highly important, with cells enduring prolonged hypometabolism being highly sensitive and susceptible to apoptosis resulting from lowered energy states. Regulating cell death is the ortholog of the p53 tumor suppressor, Cep-1 (Derry et al., 2001), which is likely to be closely regulated throughout the dauer period in an organism that does not respond well to stress induced levels of apoptosis. In conjunction with this is the cellular recycling process of autophagy which is triggered by caloric restriction and minimizes cells, allowing their survival through low energy periods (Kourtis and Tavernarakis, 2009). In preparation for the dauer stage, autophagy proteins accumulate, and autophagy is necessary for dauer survival (Morck and Pilon, 
2007; Melendez et al., 2003). Dauer, also known as a period of 'agelessness', also imposes numerous controls on the cell cycle to prevent cell division throughout the hypometabolic period (Van den Heuvel, 2005). Cellular quiescence is a common mechanism, triggered by extensive periods of stress, and is co-ordinated through regulation of multiple cell-cycle progression genes (Biggar and Storey, 2009).

2. Like Akt, loss of SGK1 in the nematode causes an extension in lifespan (dauer), but whereas Akt appears to be more critical to suppress dauer entry, it is thought that SGK-1 is more critical to development and the stress response (Hertweck et al., 2004).

3. The Smad and STAT pathways intersect through the STAT ortholog (STA-1), which regulates larval development in part through an interaction with TGF- $\beta$. STAT and TGF- $\beta$ signaling occurs in the same cell/tissue types in the worm, indicating parallel strands of developmental signaling, and may both be categorized as anti-dauer (i.e. anti-stasis) responses (Wang and Levy, 2006). Despite this, neither path can be said to contribute to dauer determinancy, but expression of STA-1 throughout the dauer period may hint at a compensatory mechanism given the severe reduction of TGF- $\beta$ signaling during the dauer stage. The two paths physically interact through two STA-1 mediated interactions, both counter to the mechanics of dauer entry (FIG. 3.2). The first mechanism, straying from the largely canonical TGF- $\beta$ signaling, involves STA-1 interacting with the type I receptor and downstream Smad (Wang and Levy, 2006). This interaction may compensate for an absence of TGF- $\beta$ ligand, type II receptor and other Smad 
members. The second mechanism involves a feedback loop. The STA-1 promoter sequence contains Smad binding regions, interestingly, which allows the prodauer Smads to up-regulate STA-1 transcription and negatively regulate dauer progression (Wang and Levy, 2006). With increased transcription of STA-1, the anti-dauer response from the first mechanism can be employed.

4. During entry into the hypometabolic dauer state, pro-development pathways like TGF- $\beta$ signaling and JAK-STAT signaling are seen as anti-dauer and are largely suppressed in order to promote cellular senescence (Wang and Levy, 2006). The STAT ortholog, STA-1 is found in most of the same tissue sets as TGF- $\beta$, so there is a strong chance of parallel expression (Wang and Levy, 2006). In the nematode, the two paths and their dauer repression responses manifest in potential mechanisms of co-operation. It is suggested that, potentially in a compensatory mechanism for the absence of TGF- $\beta$ ligand, Type II receptor and the Smad ortholog DAF-14, STA-1 is able to interact with the type I receptor and downstream Smad (DAF-8) to initiate anti-dauer, pro-development signaling (Wang and Levy, 2006). In a second mechanism, possibly for dauer exit, the prodauer Smads are able to transcriptionally up-regulate STA-1, and in doing so, also promote development (Wang and Levy, 2006).

5. The nematode C. elegans, can enter a hypometabolic period of diapause (called the 'dauer' stage) when environmental conditions are poor. Under these conditions, the $\mathrm{p} 53$ ortholog (named CEP-1) needs to be strictly regulated to avoid ectopic apoptotic cell death. It is well known that CEP-1 inactivation correlates with increased lifespan, but how this may correlate with dauer is not 
currently explained. During the dauer period, while there is currently no detailed mechanism, it may be suspected that posttranslational control, in this case by sirtuin (SIR-2.1) mediated deacetylation, could be used destabilize the p53 ortholog and, as such, extend nematode lifespan (Greiss et al., 2009). Along with this, one of the primary mediators of dauer entry, transcription factor DAF-16 (a FoxO ortholog), is also suspected to repress p 53 activity (although though, unlikely directly) (Arum and Johnson, 2007), which would add further weight to the concept of active $\mathrm{p} 53$ repression under hypometabolic conditions.

6. In the hypometabolic dauer period that occurs at the second larval stage, triggered by amongst other things, reduced oxygen and nutrient deprivation, the cell cycle is also arrested (Van den Heuvel, 2005; Wińska et al., 2005). Attenuation of the C. elegans cell cycle appears to involve CDK inhibitor induction.

7. The embryos of zebra fish (Danio rerio), can survive short bouts (24h) of anoxia, in which cell division ceases as part of a total developmental senescence (Padilla and Roth, 2001). Blastomeres arresting in S and G2 phases were thought to be a mechanism of energetic homeostasis (Padilla and Roth, 2001). Similarly, fruit fly (Drosophila melanogaster) embryonic cells were also seen to attenuate division upon oxygen deprivation (Douglas et al., 2001). Two specific oxygen sensitive checkpoints, at interphase and metaphase, were discovered in response to hypoxia, synchronized with cyclin B degradation (Douglas et al., 2001). Also like in the zebra fish study, the duration of oxygen deprivation was short (a maximum of $2 \mathrm{~h}$ exposure; Douglas et al., 2001), and as such are not necessarily relatable to environmental stresses that either animal would encounter. Conversely, encysted 
embryos of the brine shrimp (Artemia franciscana) are capable of enduring up to four years of continuous anoxia, entering an extreme diapause that would prevent cellular division (Clegg, 1997). 


\section{Appendix C: List of Publications}

\section{Published:}

Lant, B and K.B. Storey. An overview of stress response and hypometabolic strategies in Caenorhabditis elegans: conserved and contrasting signals with the mammalian system. Int J Biol Sci. 2010 Jan 7; 6(1):9-50. Review.

\section{Submitted:}

Lant, B and K.B. Storey. The role of glucose-6-phosphate dehydrogenase regulation in the anoxia tolerance of the freshwater crayfish Orconectes virilis.

Lant, B., Storey, J.M., Brooks, S.P.J. and K.B. Storey. Metabolic responses to anoxia by freshwater crayfish, Orconectes virilis.

In Preparation:

Lant, B. and K.B. Storey. Regulation of the insulin/IGF pathway in an anoxia resistant freshwater crayfish.

Lant, B. and K.B. Storey. Regulating cell death: how the freshwater crayfish (Orconectes virilis) mediates p53 and autophagy under anoxic conditions. 


\section{Appendix D: Communications at scientific meetings}

\section{Poster presentations:}

Lant, B. and K.B. Storey. P53 in the anoxia resistant crayfish: a surprising role in cellular survival. Ottawa Carleton Institute of Biology Symposium ( $7^{\text {th }}$ Annual). Ottawa, ON. April 2010.

Lant, B. and K.B. Storey. Anoxia induces autophagy in freshwater crayfish. Canadian Society of Zoology (48 ${ }^{\text {th }}$ Annual) Meeting. Toronto, ON. May 2009.

Lant, B. and K.B. Storey. Anoxia induces autophagy in freshwater crayfish. Ottawa Carleton Institute of Biology Symposium (6 ${ }^{\text {th }}$ Annual). Ottawa, ON. April 2009.

Lant, B. and K.B. Storey. Regulation of the unfolded protein response in the anoxia tolerant marine periwinkle, Littorina littorea, contributes to cellular survival during winter. Ottawa Carleton Institute of Biology Symposium ( $5^{\text {th }}$ Annual). Ottawa, ON. April 2008 .

Lant, B. and K.B. Storey. Regulation of the unfolded protein response in the anoxia tolerant marine periwinkle, Littorina littorea, contributes to cellular survival during winter. Concordia Chemistry and Biochemistry Graduate Research Conference $\left(10^{\text {th }}\right.$ Annual). Montreal, QC. November 2007.

Lant, B. and K.B. Storey. Metabolic regulation of the crayfish, Orconectes virilis: G6PDH in anoxia. Concordia Chemistry and Biochemistry Graduate Research Conference ( $9^{\text {th }}$ Annual). Montreal, QC. November 2006.

\section{Invited Symposium Presentations:}

Lant, B. and K.B. Storey. The contributions of p53 and autophagy to the survival of the anoxia resistant crayfish, Orconectes virilis. Canadian Society for Life Sciences Research ( $5^{\text {th }}$ Annual) Conference. Montreal, QC. August 2010.

\section{Other Oral Presentations:}

Lant, B. and K.B. Storey. Lessons in antioxidant defense from the anoxia resistant crayfish. Canadian Society of Zoology (50 ${ }^{\text {th }}$ Annual) Meeting. Ottawa, ON. May 2011.

Lant, B. and K.B. Storey. The critical role of autophagy: cellular rescue under extreme conditions. Concordia Chemistry and Biochemistry Graduate Research Conference $\left(12^{\text {th }}\right.$ Annual). Montreal, QC. November, 2009. 


\section{Appendix E: Ensuring antibody specificity for western blotting}

Throughout this thesis, several steps were taken to ensure that the correct band was quantified for western blotting. These include:

1. Blocking with milk/PVA before probing with the antibody was optimized to minimize any non-specific binding and ensure that only one band appeared at the expected molecular weight.

2. If available, blocking peptides were used to prove specificity of target banding, in cases of multiple banding in a particular molecular weight region.

3. In case where antibodies were tested on several different animals by the company, antibodies that were shown to cross-react well with a wide number of different species were selected. For example, the histone $\mathrm{H} 3$ antibody used cross-reacted with animals ranging from mammals to fruit flies.

4. If antibodies for different regions of the protein were available from the company, the amino acid sequences of these regions were aligned with sequences from several vertebrates and antibodies specific to the best conserved regions were selected.

5. In cases of antibodies producing consistently poor banding resolution (such as mTOR), immunoprecipitation was used to isolate the protein, and provide a quantifiable signal. 


\section{REFERENCES}


Aiken DE. Distribution and ecology of three species of crayfish from New Hampshire. $A m$ Midl Nat. 1965. 73(1): 240-4

Aiken DE. The crayfish Orconetes virilis: survival in a region with severe winter conditions. Can J Zool. 1967. 46;207-11

Akman HO, Zhang H, Siddiqui MA, Solomon W, Smith EL, Batuman OA. Response to hypoxia involves transforming growth factor-beta2 and Smad proteins in human endothelial cells. Blood. 2001. 98(12);3324-31

Alenzi FQ, Lotfy M, Wyse R. Swords of cell death: caspase activation and regulation. Asian Pac. J. Cancer Prev. 2010. 11(2);271-80

Alessi DR, Andjelkovic M, Caudwell B, Cron P, Morrice N, Cohen P, Hemmings BA. Mechanism of activation of protein kinase $\mathrm{B}$ by insulin and IGF-1. EMBO J. 1996. 15(23);6541-51

Ali A, Hoeflich KP, Woodgett JR. Glycogen synthase kinase-3: properties, functions, and regulation. Chem Rev. 2001. 101(8);2527-40

Altun ZF, Hall DH. Handbook of C. elegans Anatomy. In WormAtlas. 2005. http://www.wormatlas.org/ver1/handbook/contents.htm

Amaral JD, Xavier JM, Steer CJ, Rodrigues CM. Targeting the p53 pathway of apoptosis. Curr. Pharm. Des. 2010. 16(22);2493-503

Amati B, Alevizopoulos K, Vlach J. Myc and the cell cycle. Front Biosci. 1998. 3;250-68

Andjelkovic M, Alessi DR, Meier R, Fernandez A, Lamb NJ, Frech M, Cron P, Cohen P, Lucocq JM, Hemmings BA. Role of translocation in the activation and function of protein kinase B. J Biol Chem. 1997. 272;31515-24

Arum O, Johnson TE. Reduced expression of the Caenorhabditis elegans p53 ortholog cep-1 results in increased longevity. J. Gerontol. 2007. 62(9);951-9

Atfi A, Baron R. p53 brings a new twist to the Smad signaling network. Sci Signal. 2008. 1(26);pe33

Bahassi el M, Hennigan RF, Myer DL, Stambrook PJ. Cdc25C phosphorylation on serine 191 by Plk3 promotes its nuclear translocation. Oncogene. 2004. 23(15);2658-63

Bai X, Jiang Y. Key factors in mTOR regulation. Cell Mol Life Sci. 2010. 67(2);239-53 
Balaburski GM, Hontz RD, Murphy ME. p53 and ARF: unexpected players in autophagy. Trends Cell Biol. 2010. 20(6);363-9

Beall MJ, Pearce EJ. Transforming growth factor-beta and insulin like signaling pathways in parasitic helminthes. Intl J Parasit. 2002. 32;399-404

Bellé R, Cormier P, Poulhe R, Morales J, Huchon D, Mulner-Lorillon O. Protein phosphorylation during meiotic maturation of Xenopus oocytes: cdc2 protein kinase targets. Int J Dev Biol. 1990. 34(1);111-5

Bergamini E. Autophagy: a cell repair mechanism that retards ageing and age-associated diseases and can be intensified pharmacologically. Mol Aspects Med. 2006. 27(56);403-10

Berthet C, Kaldis P. Cell-Specific Responses to Loss of Cyclin-Dependent Kinases. Oncogene. 2007. 26;4469-77

Bhattacharya S, Eckner R, Grossman S, Oldread E, Arany Z, D'Andrea A, Livingston DM. Cooperation of Stat 2 and p300/CBP in signaling induced by interferon-alpha. Nature. 1996. 383(6598);344-7.

Biggar KK, Storey KB. Perspectives in cell cycle regulation: lessons from an anoxic vertebrate. Curr Genomics. 2009. 10(8);573-84

Biggar KK, Storey KB. The emerging roles of microRNAs in the molecular responses of metabolic rate depression. J Mol Cell Biol. 2011. 3(3);167-75

Bluyssen HA, Levy DE. Stat2 is a transcriptional activator that requires sequence-specific contacts provided by stat 1 and $\mathrm{p} 48$ for stable interaction with DNA. $J$ Biol Chem. 1997. 272(7);4600-5

Brunet A, Park J, Tran H, Hu LS, Hemmings BA, Greenberg ME. Protein kinase SGK mediates survival signals by phosphorylating the forkhead transcription factor FKHRLl (FOXO3a). Mol Cell Biol. 2001. 21; 952-5.

Burgering BMT, Kops GJPL. Cell cycle and death control: long live forkheads. Trends Biochem Sci. 2002. 27(7);352-60

Buzzai M, Bauer DE, Jones RG, Deberardinis RJ, Hatzivassiliou G, Elstrom RL, Thompson $\mathrm{CB}$. The glucose dependence of Akt-transformed cells can be reversed by pharmacologic activation of fatty acid beta-oxidation. Oncogene. 2005. 24(26);416573

Cardoso MC, Leonhardt H, Nadal-Ginard B. Reversal of terminal differentiation and control of DNA replication: cyclin A and Cdk2 specifically localize at subnuclear sites of DNA replication. Cell. 1993. 74(6);979-92 
Casamayor A, Morrice NA, Alessi DR. Phosphorylation of Ser-241 is essential for the activity of 3-phosphoinositide-dependent protein kinase-1: identification of five sites of phosphorylation in vivo. Biochem J. 1999. 342(2);287-92

Chen WY, Ho KC, Leu JH, Liu KF, Wang HC, Kou GH, Lo CF. WSSV infection activates STAT in shrimp. Dev Comp Immunol. 2008. 32(10);1142-50

Choudhury A, Cuddihy A, Bristow RG. Radiation and new molecular agents part I: targeting ATM-ATR checkpoints, DNA repair, and the proteasome. Semin Radiat Oncol. 2006. $16(1) ; 51-8$

Clayton JE, van den Heuvel SJ, Saito RM. Transcriptional control of cell-cycle quiescence during C. elegans development. Dev Biol. 2008. 313(2);603-13

Clegg J. Embryos of Artemia franciscana survive four years of continuous anoxia: the case for complete metabolic rate depression. J Exp Biol. 1997. 200(Pt 3);467-75

Cowan KJ, Storey KB. Protein kinase and phosphatase responses to anoxia in crayfish, Orconectes virilis: purification and characterization of cAMP-dependent protein kinase. Comp Biochem Physiol B Biochem Mol Biol. 2001. 130(4);565-77

Cowan KJ. 1998. Evidence for reversible phosphorylation control of metabolism during prolonged environmental stress. Ph.D. thesis, Dept. Chemistry, Carleton University

Crighton D, Wilkinson S, Ryan KM. DRAM links autophagy to p53 and programmed cell death. Autophagy. 2007 Jan-Feb;3(1):72-4. Epub 2007 Jan 28.

Crighton D, Wilkinson S, Ryan KM. DRAM links autophagy to p53 and programmed cell death. Autophagy. 2007. 3(1);72-4

Crocker DW, Barr DW. Handbook of the crayfishes of Ontario. 1968. University of Toronto Press. Pp: 26-30 and 91-102

da Silva-Castiglioni D, Oliveira GT, Buckup L. Metabolic responses of Parastacus defossus and Parastacus brasiliensis (Crustacea, Decapoda, Parastacidae) to hypoxia. Comp Biochem Physiol A Mol Integr Physiol. 2010. 156(4);436-44

da Silva-Castiglioni D, Oliveira GT, Buckup L. Metabolic responses in two species of crayfish (Parastacus defossus and Parastacus brasiliensis) to post-hypoxia recovery. Comp Biochem Physiol A Mol Integr Physiol. 2011. 159(3);332-8

Daniels SA, Ailion M, Thomas JH, Sengupta P. Egl-4 acts through a transforming growth factor $\beta / \mathrm{SMAD}$ pathway in Caenorhabditis elegans to regulate multiple neuronal circuits in response to sensory cues. Genetics. 2000. 156;123-41 
Dawson NJ, Storey KB. Regulation of tail muscle arginine kinase by reversible phosphorylation in an anoxia-tolerant crayfish. J Comp Physiol B. 2011. Apr 26. [Epub ahead of print]

Dearolf CR. JAKs and STATs in invertebrate model organisms. Cell Mol Life Sci. 1999. $55(12) ; 1578-84$

DeBerardinis RJ, Lum JJ, Hatzivassiliou G, Thompson CB. The biology of cancer: metabolic reprogramming fuels cell growth and proliferation. Cell Metab. 2008. 7(1);11-20

Dechend R, Hirano F, Lehmann K, Heissmeyer V, Ansieau S, Wulczyn FG, Scheidereit C, Leutz A. The Bcl-3 oncoprotein acts as a bridging factor between NF-kappaB/Rel and nuclear co-regulators. Oncogene. 1999. 18(22):3316-23

Deng X, Ruvolo P, Carr B, May WS Jr. Survival function of ERK1/2 as IL-3-activated, staurosporine-resistant Bcl2 kinases. Proc Natl Acad Sci U S A. 2000. 97(4);1578-83

Deng X, Xiao L, Lang W, Gao F, Ruvolo P, May WS Jr. Novel role for JNK as a stressactivated Bcl2 kinase. J Biol Chem. 2001. 276(26);23681-8

Denlinger DL. Regulation of diapause. Annu Rev Entomol.2002. 47;93-122

Derry WB, Putzke AP, Rothman JH. Caenorhabditis elegans p53: role in apoptosis, meiosis, and stress resistance. Science. 2001. 294(5542);591-5

Díaz-Troya S, Pérez-Pérez ME, Florencio FJ, Crespo JL. The role of TOR in autophagy regulation from yeast to plants and mammals. Autophagy. 2008. 4(7);851-65

Dimmeler S, Breitschopf K, Haendeler J, Zeiher AM. Dephosphorylation targets Bcl-2 for ubiquitin-dependent degradation: a link between the apoptosome and the proteasome pathway. J Exp Med. 1999. 189(11);1815-22

Douglas RM, Xu T, Haddad GG. Cell cycle progression and cell division are sensitive to hypoxia in Drosophila melanogaster embryos. Am J Physiol Regul Integr Comp Physiol. 2001. 280(5);1555-63

Duckworth BC, Weaver JS, Ruderman JV. G2 arrest in Xenopus oocytes depends on phosphorylation of cdc25 by protein kinase A. Proc Natl Acad Sci U S A. 2002. 99(26);16794-9

Eskelinen EL. New insights into the mechanisms of macroautophagy in mammalian cells. Int Rev Cell Mol Biol. 2008. 266;207-47 
Ferecatu I, Bergeaud M, Rodríguez-Enfedaque A, Le Floch N, Oliver L, Rincheval V, Renaud F, Vallette FM, Mignotte B, Vayssière JL. Mitochondrial localization of the low level p53 protein in proliferative cells. Biochem. Biophys. Res. Commun. 2009. 387(4);772-7

Ferraro E, and Cecconi F. Autophagic and apoptotic response to stress signals in mammalian cells. Arch. Biochem. Biophys. 2007. 462;210-19

Fukuyama M, Gendreau SB, Derry WB, Rothman JH. Essential embryonic roles of the CKI1 cyclin-dependent kinase inhibitor in cell-cycle exit and morphogenesis in $\mathrm{C}$ elegans. Dev Biol. 2003. 260(1);273-86

Fukuyama M, Rougvie AE, and Rothman JH. C. elegans Daf-18/PTEN mediates nutrientdependent arrest of cell cycle growth in the germline. Curr Biol. 2006. 16(8);773-9

Furnari B, Blasina A, Boddy MN, McGowan CH, Russell P. Cdc25 inhibited in vivo and in vitro by checkpoint kinases Cds1 and Chk1. Mol Biol Cell. 1999. 10(4);833-45

Gallo M, Park D, Luciani DS, Kida K, Palmieri F, Blacque OE, Johnson JD, Riddle DL. MISC-1/OGC links mitochondrial metabolism, apoptosis and insulin secretion. PLoS One. 2011. 6(3);e17827

Gartel AL, Shchors K. Mechanisms of c-myc-mediated transcriptional repression of growth arrest genes. Exp Cell Res. 2003. 283(1);17-21

Gems D, Sutton AJ, Sundermeyer ML, Albert PS, King KV, Edgley ML, Larsen PL, Riddle DL. Two pleiotropic classes of Daf-2 mutation affect larval arrest, adult behavior, reproduction and longeivity in Caenorhabditis elegans. Genetics. 1998. 150(1);12955

Goto T, Takano M. Transcriptional role of FOXO1 in drug resistance through antioxidant defense systems. Adv Exp Med Biol. 2009. 665:171-9

Gray JV, Petsko GA, Johnston GC, Ringe D, Singer RA, Werner-Washburne M. "Sleeping beauty": quiescence in Saccharomyces cerevisiae. Microbiol Mol Biol Rev. 2004. 68(2);187-206

Green DR, Kroemer G. Cytoplasmic functions of the tumour suppressor p53. Nature. 2009. 458(7242);1127-30

Greiss S, Hall J, Ahmed S, Gartner A. C elegans SIR-2.1 translocation is linked to a proapoptotic Pathway parallel to cep-1/p53 during DNA damage-induced apoptosis. Genes Dev. 2009. 22:2831-42

Gu Z, Flemington C, Chittenden T, Zambetti GP. ei24, a p53 response gene involved in growth suppression and apoptosis. Mol Cell Biol. 2000. 20(1);233-41 
Guppy M, Withers P. Metabolic depression in animals: physiological perspectives and biochemical generalizations. Biol Rev Camb Philos Soc. 1999. 74(1);1-40

Hammond EM, Dorie MJ, Giaccia AJ. ATR/ATM targets are phosphorylated by ATR in response to hypoxia and ATM in response to reoxygenation. J. Biol. Chem. 2003. $278 ; 12207-13$

Hammond EM, Giaccia AJ. The role of p53 in hypoxia-induced apoptosis. Biochem. Biophys. Res. Commun. 2005. 331(3):718-25

Hanada T, Noda NN, Satomi Y, Ichimura Y, Fujioka Y, Takao T, Inagaki F, Ohsumi Y. The Atg12-Atg5 conjugate has a novel E3-like activity for protein lipidation in autophagy. $J$ Biol Chem. 2007. 282(52);37298-302

Hannon GJ, Casso D, Beach D. KAP: a dual specificity phosphatase that interacts with cyclin-dependent kinases. Proc Natl Acad Sci US A. 1994. 91(5);1731-5

He C, Levine B. The Beclin 1 interactome. Curr Opin Cell Biol. 2010. 22(2);140-9

Heald R, McLoughlin M, McKeon F. Human weel maintains mitotic timing by protecting the nucleus from cytoplasmically activated Cdc2 kinase. Cell. 1993. 74(3);463-74

Hermes-Lima M, Storey JM, Storey KB. Antioxidant defenses and metabolic depression. The hypothesis of preparation for oxidative stress in land snails. Comp Biochem Physiol B. 1998. 120;437-48

Hermes-Lima M, Zenteno-Savín T. Animal response to drastic changes in oxygen availability and physiological oxidative stress. Comp. Biochem. Physiol. C. 2002. 133(4);537-56

Hertweck M, Göbel C, Baumeister R. C. elegans SGK-1 is the critical component in the Akt/PKB kinase complex to control stress response and life span. Dev Cell. 2004. $6(4) ; 577-88$.

Hochachka PW. Balancing conflicting metabolic demands of exercise and diving. Fed Proc. 1986. 45(13);2948-52

Holdich DM, Lowery RS. Freshwater crayfish: biology, management and exploitation.1988. Timber press, Portland, OR. Pp.262-9

Huang H, Tindall DJ. Dynamic FoxO transcription factors. J Cell Sci. 2007. 120(Pt15);247987.

Huang WP, Scott SV, Kim J, Klionsky DJ. The itinerary of a vesicle component, Aut $7 \mathrm{p} / \mathrm{Cvt} 5 \mathrm{p}$, terminates in the yeast vacuole via the autophagy/Cvt pathways. $J$ Biol Chem. 2000. 275(8);5845-51 
Inman GJ. Switching TGF $\beta$ from a tumor suppressor to a tumor promoter. Curr Opin Genet Dev. 2011. 21(1);93-9

Inoue T, Thomas JH. Targets of TGF- $\beta$ signaling in Caenorhabditis elegans dauer formation. Dev Biol. 2000. 217;192-204

Inoue Y, Itoh Y, Abe K, Okamoto T, Daitoku H, Fukamizu A, Onozaki K, Hayashi H. Smad3 is acetylated by $\mathrm{p} 300 / \mathrm{CBP}$ to regulate its transactivation activity. Oncogene. 2007. 26(4);500-8

Jackman M, Kubota Y, den Elzen N, Hagting A, Pines J. Cyclin A- and cyclin E-Cdk complexes shuttle between the nucleus and the cytoplasm. Mol Biol Cell. 2002. $13(3) ; 1030-45$

Jackson DC, Wang T, Koldkjaer P, Taylor EW. Lactate sequestration in the carapace of the crayfish Austropotamobius pallipes during exposure in air. J Exp Biol. 2001. 204(Pt 5);941-6

Jackson DC, Taylor SE, Asare VS, Villarnovo D, Gall JM, Reese SA. Comparative shell buffering properties correlate with anoxia tolerance in freshwater turtles. $A m J$ Physiol Regul Integr Comp Physiol. 2007. 292(2);1008-15

Jiang BH, Liu LZ. PI3K/PTEN signaling in tumorigenesis and angiogenesis. Biochim Biophys Acta. 2008. 1784;150-8

Karantza-Wadsworth V, Patel S, Kravchuk O, Chen G, Mathew R, Jin S, White E. Autophagy mitigates metabolic stress and genome damage in mammary tumorigenesis. Genes Dev. 2007. 21(13);1621-35

Kerr JF, Wyllie AH, Currie AR. Apoptosis: a basic biological phenomenon with wideranging implications in tissue kinetics. Br J Cancer. 1972. 26(4);239-57

Koff A, Giordano A, Desai D, Yamashita K, Harper JW, Elledge S, Nishimoto T, Morgan DO, Franza BR, Roberts JM. Formation and activation of a cyclin E-cdk2 complex during the G1 phase of the human cell cycle. Science. 1992. 257(5077);1689-94

Korenjak M, Brehm A. E2F-Rb complexes regulating transcription of genes important for differentiation and development. Curr Opin Genet Dev. 2005. 15(5);520-7

Kourtis N, Tavernarakis N. Autophagy and cell death in model organisms. Cell Death and Diff. 2009. 16;21-30

Krieger C, Duchen MR. Mitochondria, Ca2+ and neurodegenerative disease. Eur $J$ Pharmacol. 2002. 447(2-3);177-88 
Krivoruchko A, Storey KB. Activation of antioxidant defenses in response to freezing in freeze-tolerant painted turtle hatchlings. Biochim Biophys Acta. 2010. 1800(7);662-8

Krivoruchko A, Storey KB. Forever young: mechanisms of natural anoxia tolerance and potential links to longevity. Oxid Med Cell Longev. 2010. 3(3);186-98

Kumagai A, Dunphy WG. Binding of 14-3-3 proteins and nuclear export control the intracellular localization of the mitotic inducer Cdc25. Genes Dev. 1999. 13(9);106772

Ladu S, Calvisi DF, Conner EA, Farina M, Factor VM, Thorgeirsson SS. E2F1 Inhibits cMYC Driven Apoptosis via PIK3CA/Akt/mTOR and Cox-2 on a Mouse Model of Human Liver Cancer. Gastroenterology. 2008. 135(4);1322-32

Laemmli UK. Cleavage of structural proteins during the assembly of the head of bacteriophage T4. Nature. 1970. 227(5259);680-85

Lant B, Storey KB. An overview of stress response and hypometabolic strategies in Caenorhabditis elegans: conserved and contrasting signals with the mammalian system. Int J Biol Sci. 2010. 6(1);9-50

Lavin MF, Gueven N. The Complexity of p53 Stabilization and Activation. Cell Death Diff. 2006; 13: $941-50$

Lee SW, Reimer CL, Fang L, Iruela-Arispe ML, Aaronson SA. Overexpression of kinaseassociated phosphatase (KAP) in breast and prostate cancer and inhibition of the transformed phenotype by antisense KAP expression. Mol Cell Biol. 2000. 20(5);1723-32

Levine B, Sinha S, Kroemer G. Bcl-2 family members: dual regulators of apoptosis and autophagy. Autophagy. 2008. 4(5);600-6

Li M, Brooks CL, Wu-Baer F, Chen D, Baer R, Gu W. Mono- versus polyubiquitination: differential control of p53 fate by Mdm2. Science. 2003. 302(5652);1972-5

Liang J, Slingerland JM. Multiple roles of the PI3K/PKB (Akt) pathway in cell cycle progression. Cell Cycle. 2003. 2(4);339-45

Lishner M, Lalkin A, Klein A, Yarkoni S, Manor Y, Fejgin M, Leytin V, Ravid M, Amiel A. The BCL-1, BCL-2, and BCL-3 oncogenes are involved in chronic lymphocytic leukemia. Detection by fluorescence in situ hybridization. Cancer Genet Cytogenet. 1995. 85(2);118-23

Liu D, Yang X, Songyang Z. Identification of CISK, a new member of the SGK kinase family that promotes IL-3-dependent survival. Curr Biol. 2000. 10;1233-36. 
Long J, Matsuura I, He D, Wang G, Shuai K, Liu F. Repression of Smad transcriptional activity by PIASy, an inhibitor of activated STAT. Proc Natl Acad Sci U S A. 2003. 100(17);9791-6

Lundberg AS, Weinberg RA. Functional inactivation of the retinoblastoma protein requires sequential modification by at least two distinct cyclin-cdk complexes. Mol Cell Biol. 1998. 18;753-61

MacDonald JA, Storey KB. Regulation of ground squirrel $\mathrm{Na}^{+} \mathrm{K}^{+}$-ATPase activity by reversible phosphorylation during hibernation. Biochem Biophys Res Commun. 1999. 254(2);424-9

Mahony D, Parry DA, Lees E. Active cdk6 complexes are predominantly nuclear and represent only a minority of the cdk6 in T cells. Oncogene. 1998. 16(5);603-11

Maiese K, Hou J, Chong ZZ, Shang YC. A fork in the path: developing therapeutic inroads with FoxO proteins. Oxid Med Cell Longev. 2009. 2(3):119-29

Maiuri MC, Le Toumelin G, Criollo A, Rain JC, Gautier F, Juin P, Tasdemir E, Pierron G, Troulinaki K, Tavernarakis N, Hickman JA, Geneste O, Kroemer G. Functional and physical interaction between $\mathrm{Bcl}-\mathrm{X}(\mathrm{L})$ and a $\mathrm{BH} 3$-like domain in Beclin-1. EMBO J. 2007. 26(10);2527-39

Malhotra R, Lin Z, Vincenz C, Brosius FC 3rd. Hypoxia induces apoptosis via two independent pathways in Jurkat cells: differential regulation by glucose. Am J Physiol Cell Physiol. 2001. 281(5);C1596-603

Malik AI, Storey KB. Transcriptional regulation of antioxidant enzymes by FoxO1 under dehydration stress. Gene. 2011. Jun 25. [Epub ahead of print]

Maridor G, Gallant P, Golsteyn R, Nigg EA. Nuclear localization of vertebrate cyclin A correlates with its ability to form complexes with cdk catalytic subunits. J Cell Sci. 1993. 106(Pt2);535-44

Massagué J, Seoane J, Wotton D. Smad transcription factors. Genes Dev. 2005. 19;2783-810

Mazia, D. Biochemistry of the dividing cell. Annu Rev Biochem. 1962. 30;669-88

McMahon BR. Respiratory and circulatory compensation to hypoxia in crustaceans. Respir Physiol. 2001. 128(3);349-64

McMullen DC, Storey KB. Suppression of $\mathrm{Na}^{+} \mathrm{K}^{+}$-ATPase activity by reversible phosphorylation over the winter in a freeze-tolerant insect. $J$ Insect Physiol. 2008. 54(6);1023-7 
Meek DW. Tumour suppression by p53: a role for the DNA damage response? Nat. Rev. Cancer. 2009. 9(10); 714-23

Melendez A, Talloczy Z, Seaman M, Eskelinen EL, Hall DH, Levine B. Autophagy genes are essential for dauer development and life-span extension in C. elegans. Science. 2003. 301;1387-91

Menze MA, Fortner G, Nag S, Hand SC. Mechanisms of apoptosis in Crustacea: What conditions induce versus suppress cell death? Apoptosis. 2010. 15(3);293-312

Mihara M, Erster S, Zaika A, Petrenko O, Chittenden T, Pancoska P, Moll UM. p53 has a direct apoptogenic role at the mitochondria. Mol. Cell. 2003. 11(3);577-90

Miyazono K. Molecular mechanisms of transforming growth factor- $\beta$ signaling and disease: The 59th Fujihara International Seminar, 2010. Cancer Sci. 2011. 102(6);1242-4

Mörck C, Pilon M. Caloric restriction and autophagy in Caenorhabditis elegans. Autophagy. 2007. 3(1);51-3

Moskalev AA. Genetic investigations of low dose irradiation influence on life span. Radiat Biol Radioecol. 2008. 48(2);139-45

Mukhopadhyay A, Oh SW, Tissenbaum HA. Worming pathways to and from DAF16/FOXO. Exp Gerontol. 2006. 41(10);928-34

Nakatsuji T, Lee CY, Watson RD. Crustacean molt-inhibiting hormone: structure, function, and cellular mode of action. Comp Biochem Physiol A Mol Integr Physiol. 2009. 152(2);139-48

Narasimhan SD, Yen K, Bansal A, Kwon ES, Padmanabhan S, Tissenbaum HA. PDP-1 links the TGF- $\beta$ and IIS pathways to regulate longevity, development, and metabolism. PLoS Genet. 2011. 7(4);1001377

Nobukuni T, Kozma SC, Thomas G. hvps34, an ancient player, enters a growing game: mTOR complex1/S6K1 signaling. Curr Opin Cell Biol. 2007. 19;135-41

Obaya AJ, Sedivy JM. Regulation of cyclin-Cdk activity in mammalian cells. Cell Mol Life Sci. 2002. 59(1);126-42

Ohtsubo M, Theodoras AM, Schumacher J, Roberts JM, Pagano M. Human Cyclin E, a Nuclear Protein Essential for the G1-to-S Phase Transition. Mol Cell Biol. 1995. 15(5);2612-24

Oidtmann B, Stentiford GD. White spot syndrome virus (WSSV) concentrations in crustacean tissues - a review of data relevant to assess the risk associated with commodity trade. Transbound Emerg Dis. 2011 May 29 [Epub ahead of print] 
Olcina M, Lecane PS, Hammond EM. Targeting hypoxic cells through the DNA damage response. Clin Cancer Res. 2010. 16(23);5624-9

Osaki M, Oshimura M, Ito H. PI3K-Akt pathway: its functions and alterations in human cancer. Apoptosis. 2004. 9;667-76

Padgett RW, Das P, Krishna S. TGF-beta signaling, Smads, and tumor suppressors. Bioessays. 1998. 20(5);382-90

Padilla PA, Roth MB. Oxygen deprivation causes suspended animation in the zebrafish embryo. Proc Natl Acad Sci U S A. 2001. 98(13);7331-5

Paradis S, Ailion M, Toker A, Thomas JH, Ruvkun G. A PDK1 homolog is Necessary and sufficient to transduce AGE-1 PI3 kinase signals that regulate diapause in Caenorhabditis elegans. Genes Dev. 2009. 13;1438-52

Pattingre S, Espert L, Biard-Piechaczyk M, Codogno P. Regulation of macroautophagy by mTOR and Beclin 1 complexes. Biochimie. 2008. 90;313-23

Pimkina J, Humbey O, Zilfou JT, Jarnik M, Murphy ME. ARF induces autophagy by virtue of interaction with Bcl-xl. J Biol Chem. 2009. 284(5);2803-10

Porter LA, Donoghue DJ. Cyclin BI and CDK1: nuclear localization and upstream regulators. Prog Cell Cycle Res. 2003, 5;335-47

Proud CG. Signaling to translation: how signal transduction pathways control the protein synthetic machinery. Biochem J. 2007. 403(2);217-34

Qian Y, Chen X. Tumor suppression by p53: making cells senescent. Histol. Histopathol. 2010. 25(4);515-26

Raftery LA, Sutherland DJ. TGF-beta family signal transduction in Drosophila development: from Mad to Smads. Dev Biol. 1999. 210(2);251-68

Ramnanan CJ, Groom AG, Storey KB. Akt and its downstream targets play key roles in mediating dormancy in land snails. Comp Biochem Physiol B Biochem Mol Biol. 2007. 148(3);245-55

Reiber CL. Physiological adaptations of crayfish to the hypoxic environment. Amer Zool. 1995. 35;1-11

Reinhardt HC, Yaffe MB. Kinases that control the cell cycle in response to DNA damage: Chk1, Chk2, and MK2. Curr Opin Cell Biol. 2009. 21(2);245-55 
Rinn JL, Huarte M. To repress or not to repress: This is the guardian's question. Trends Cell Biol. 2011. 21(6);344-53

Roberson ED, English JD, Adams JP, Selcher JC, Kondratick C, Sweatt JD. The mitogenactivated protein kinase cascade couples PKA and PKC to cAMP response element binding protein phosphorylation in area CA1 of hippocampus. J Neurosci. 1999. $19 ; 4337-48$

Rocha S, Martin AM, Meek DW, Perkins ND. p53 represses cyclin D1 transcription through down regulation of Bcl-3 and inducing increased association of the p52 NF-kappaB subunit with histone deacetylase 1. Mol Cell Biol. 2003. 23(13);4713-27

Rodrigues MS, Reddy MM, Sattler M. Cell cycle regulation by oncogenic tyrosine kinases in myeloid neoplasias: from molecular redox mechanisms to health implications. Antioxid Redox Signal. 2008. 10(10);1813-48

Rosenbluth JM, Mays DJ, Pino MF, Tang LJ, Pietenpol JA. A gene signature-based approach identifies mTOR as a regulator of p73. Mol. Cell Biol. 2008. 28(19):5951-64

Rössig L, Jadidi AS, Urbich C, Badorff C, Zeiher AM, Dimmeler S. Akt-dependent phosphorylation of $\mathrm{p} 21$ (Cipl) regulates PCNA binding and proliferation of endothelial cells. Mol Cell Biol. 2001. 21(16);5644-57

Rouschop KM, Wouters BG. Regulation of autophagy through multiple independent hypoxic signaling pathways. Curr Mol Med. 2009. 9(4);417-24

Samara C, Tavernarakis N. Autophagy and cell death in Caenorhabditis elegans. Curr Pharm Des. 2008. 14(2); 97-115

Sancak Y, Thoreen CC, Peterson TR, Lindquist RA, Kang SA, Spooner E, Carr SA, Sabatini DM. PRAS40 is an insulin-regulated inhibitor of the mTORCl protein kinase. $\mathrm{Mol}$ Cell. 2007. 25(6);903-15

Sano T, Higaki T, Handa K, Kadota Y, Kuchitsu K, Hasezawa S, Hoffmann A, Endter J, Zimmermann U, Hedrich R, Roitsch T. Calcium ions are involved in the delay of plant cell cycle progression by abiotic stresses. FEBS Lett. 2006. 580(2);597-602

Selye H. Stress and the general adaptation syndrome. $B r$ Med J. 1950. 1(4667);1383-92

Sherr CJ, Roberts JM. CDK Inhibitors: Positive and Negative Regulators of the G1-Phase Progression. Genes Dev. 1999. 13(12);1501-12

Shoubridge EA, Hochachka PW. Ethanol: novel end product of vertebrate anaerobic metabolism. Science. 1980. 209(4453);308-9 
Smith DJ, Ng H, Kluck RM, Nagley P. The mitochondrial gateway to cell death. Life. 2008. $60(6) ; 383-9$

Song MS, Carracedo A, Salmena L, Song SJ, Egia A, Malumbres M, Pandolfi PP. Nuclear PTEN regulates the APC-CDHl tumor-suppressive complex in a phosphataseindependent manner. Cell. 2011. 144(2);187-99

Speidel D. Transcription-independent $\mathrm{p} 53$ apoptosis: an alternative route to death. Trends Cell Biol. 2010. 20(1);14-24

Stephanou A, Latchman DS. STAT-1: a novel regulator of apoptosis. Intl J Exp Path. 2003. $84 ; 239-44$

Stephanou A. Role of STAT-1 and STAT-3 in ischaemia/reperfusion injury. J Cell Mol Med. 2004. 8(4); 519-25

Storey KB. Adventures in oxygen metabolism. Comp Biochem Physiol B Biochem Mol Biol. 2004. 139(3);359-69

Storey KB, Mosser DD, Douglas DN, Grundy JE, Storey JM. Biochemistry below $0^{\circ} \mathrm{C}$ : nature's frozen vertebrates. Braz J Med Biol Res. 1996. 29(3);283-307

Storey KB, Storey JM. Metabolic rate depression in animals: transcriptional and translational control. Biol Rev Camb Philos Soc. 2004. 79(1); 207-33

Storey KB, Storey JM. Metabolic rate depression: the biochemistry of mammalian hibernation. Adv Clin Chem. 2010a. 52;77-108

Storey KB, Storey JM. Metabolic regulation and gene expression during aestivation. Prog Mol Subcell Biol. 2010b. 49;25-45

Storz P. Forkhead homeobox type $O$ transcription factors in the responses to oxidative stress. Antioxid Redox Signal. 2011. 14(4);593-605

Swiech L, Perycz M, Malik A, Jaworski J. Role of mTOR and pathology of the nervous system. Biochim Biophys Acta. 2008. 1784;116-132

Tessier SN, Storey KB. Expression of myocyte enhancer factor-2 and downstream genes in ground squirrel skeletal muscle during hibernation. Mol Cell Biochem. 2010. 344(12);151-62

Trimarchi JM, Lees JA. Sibling rivalry in the E2F family. Nat Rev Mol Cell Biol. 2002. $3(1) ; 11-20$ 
Trotman LC, Alimonti A, Scaglioni PP, Koutcher JA, Cordon-Cardo C, Pandolfi PP. Identification of a tumour suppressor network opposing nuclear Akt function. Nature. 2006. 441(7092);523-7

Van den Heuvel S. Cell-cycle regulation. WormBook, ed. The C. elegans Research Community, WormBook, doi/10.1895/wormbook.1.28.1.2005. http://www.wormbook.org.

van den Thillart G, van Lieshout G, Storey K, Cortesi P, de Zwaan A. Influence of long-term hypoxia on the energy metabolism of the haemoglobin-containing bivalve Scapharca inaequivalvis: critical $\mathrm{O}_{2}$ levels for metabolic depression. J Comp Physiol B. 1992. 162(4);297-304

Van Gorp AC, Pomeranz KM, Birkenkamp KU, Hui RC, Lam EW, Coffer PJ. Chronic protein kinase $B(\mathrm{PKB} / \mathrm{c}-\mathrm{Akt})$ activation leads to apoptosis Induced by oxidative stress-mediated Foxo3a transcriptional up-regulation. Cancer Res. 2008. 66(22);10760-9

Vanfleteren JR, Braeckman BP. Mechanisms of life span determination in Caenorhabditis elegans. Neurobiol Aging. 1999. 20;487-502

Vaquer-Sunyer R, Duarte CM. Thresholds of hypoxia for marine biodiversity. Proc Natl Acad Sci U S A. 2008. 105(40);15452-7

Varelas X, Sakuma R, Samavarchi-Tehrani P, Peerani R, Rao BM, Dembowy J, Yaffe MB, Zandstra PW, Wrana JL. TAZ controls Smad nucleocytoplasmic shuttling and regulates human embryonic stem-cell self-renewal. Nat Cell Biol. 2008. 10(7);837-48

Vidal A, Koff A. Cell-cycle inhibitors: three families united by a common cause. Gene. 2000. 247(1-2);1-15

Wang L, Harris TE, Roth RA, Lawrence JC Jr. PRAS40 regulates mTORC1 kinase activity by functioning as a direct inhibitor of substrate binding. J Biol Chem. 2007. 282(27);20036-44

Wang Y, Levy DE. $C$. elegans STAT co-operates with DAF-7/TGF- $\beta$ signaling to repress dauer formation. Curr Biol. 2006. 16;89-94

Wang Y, Levy DE. C. elegans STAT: Evolution of a regulatory switch. FASEB J. 2006. 20(10);1641-52

Wang YH, Huang ML. Organogenesis and tumorigenesis: insight from the JAK/STAT pathway in the Drosophila eye. Dev Dyn. 2010. 239(10);2522-33

Watanabe S, Arai K. Roles of the JAK-STAT system in signal transduction via cytokine receptors. Curr Opin Genet Dev. 1996. 6(5);587-96 
Wińska P, Gołos B, Cieśla J, Zieliński Z, Fraczyk T, Wałajtys-Rode E, Rode W. Developmental arrest in Caenorhabditis elegans dauer larvae causes high expression of enzymes involved in thymidylate biosynthesis, similar to that found in Trichinella muscle larvae. Parasitology. 2005. 131(Pt 2);247-54

Wrighton KH, Lin X, Feng XH. Phospho-Control of TGF- $\beta$ Superfamily Signaling. Cell Res. 2009. 19(1);8-20

Wroble BN, Finkielstein CV, Sible JC. Wee1 kinase alters cyclin E/Cdk2 and promotes apoptosis during the early embryonic development of Xenopus laevis. BMC Dev Biol. 2007. 7;119-32

Wu MY, Hill CS. TGF- $\beta$ superfamily signaling in embryonic development and homeostasis. Dev Cell. 2009. 16;329-43

Xie Z, Nair U, Klionsky DJ. Atg8 controls phagophore expansion during autophagosome formation. Mol Biol Cell. 2008. 19(8);3290-8

Yang J, Yu Y, Hamrick HE, Duerksen-Hughes PJ. ATM, ATR and DNA-PK: initiators of the cellular genotoxic stress responses. Carcinogenesis. 2003. 10:1571-80

Yi SX, Lee RE Jr. Rapid cold-hardening blocks cold-induced apoptosis by inhibiting the activation of pro-caspases in the flesh fly Sarcophaga crassipalpis. Apoptosis. 2011. $16(3) ; 249-55$

Yorimitsu T, Klionsky DJ. Autophagy: molecular machinery for self-eating. Cell Death Differ. 2005. 12 Suppl 2;1542-52

Zhang H, Akman HO, Smith EL, Zhao J, Murphy-Ullrich JE, Batuman OA. Cellular response to hypoxia involves signaling via Smad proteins. Blood. 2003. 101(6);225360 